\title{
Research and Development Toward Massive Liquid Argon Time Projection Chambers for Neutrino Detection
}

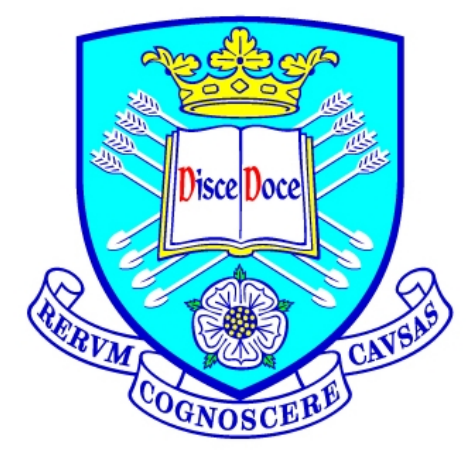

\author{
Matthew Thiesse \\ Supervisor: Prof. N. J. C. Spooner \\ Department of Physics and Astronomy \\ The University of Sheffield
}

A thesis submitted in partial fulfilment of the requirements for the degree of Doctor of Philosophy

October 2017 

To Lily. 



\section{Declaration}

I hereby declare that except where specific reference is made to the work of others, the contents of this thesis are original and have not been submitted in whole or in part for consideration for any other degree or qualification in this, or any other university. This thesis is my own work, and where work has been done in collaboration with others, my best attempts have been made to indicate this.

The information contained in Chapters 1 and 2 are summaries of the theory necessary to support this thesis. References to the works from which these theories originate, or reviews of the relevant works which include references themselves, are provided within.

Chapter 3 describes the DUNE 35-ton, a large collaboration, of which I was a member. During the run, I performed a week of monitoring and debugging shifts, and I contributed source code in LArSoft to deal with muon counter triggering. The study of North-South muon counter locations was entirely my work.

Chapter 4 describes the camera system installed in the 35-ton cryostat. I performed the camera selection and camera module design studies, as well as contributing to the characterisation. Nicola McConkey, Michael Wallbank, and Karl Warburton completed most of the system design and installation. Operation of the system during the 35-ton Phase II run was shared equally among the four of us.

Chapter 5 describes the simulation and reconstruction of the 35-ton data, which uses the LArSoft framework. My contribution to the 35-ton simulation includes the configuration and production of simulated samples and the adaptation of the Data-Driven Noise Overlay software for the 35-ton from the MicroBooNE software. I developed the Robust Hit Finder and Track Hit Backtracker algorithms.

Chapter 6 details the analysis of reconstruction performance and electron lifetime for the 35-ton. All of the work within was performed by me, with helpful comments along the way by Tom Junk, Michelle Stancari, Tingjun Yang, and Mark Convery.

Chapter 7 describes the design and construction of the LAr test stand at Sheffield. My work includes the purchasing and selection of most instrumentation components, and the initial layout of the system. I also assisted Nicola McConkey and Matthew Wright in the construction and commissioning of the system. 
Chapter 8 describes the GPM, which was built by Brais Paredes Lopez, Carlos Azevedo (University of Aveiro) and Joao Veloso (University of Aveiro). The data acquisition software was developed entirely by myself using CAEN and Keysight APIs. The preamplifier and shaping amplifier modules were assembled by Nicola McConkey. The four experiments presented were designed and completed by myself, with some assistance from Dominic Barker.

Matthew Thiesse

October 2017 


\section{Acknowledgements}

First of all, I would like to thank my supervisor, Neil Spooner, without whose support and guidance, this thesis would not have been possible.

Also, I would like to thank the many nice people at Fermilab for their supervision and helpful feedback on my work for the 35-ton including Alan Hahn, Tom Junk, Michelle Stancari, and Tingjun Yang.

I would like to thank the many helpful people in the Sheffield PPPA group and Physics Department as a whole who have assisted in solving my many problems in the lab, computing support, general academic support, and mechanical workshop support. Especially, Simon Dixon, Trevor Gamble, Paul Hodgson, Elena Korolkova, Vitaly Kudryavtsev, Nicola McConkey, John McMillan, Lee Thompson, and Matthew Wright. Without the unreserved support of these people, I would inevitably still be lost!

A huge thank you to all of my friends and colleagues who have made my time in the UK the best experience of my life! Thanks especially to the people with whom I have worked and shared offices: Mike, Karl, Andy, Anthony, Calum, Dave, Brais, Fred, Gary, Dom, Guillermo, and Warren.

Most of all, I would like to thank my amazing wife, Christy, for listening to me rant about physics and for providing unending and unconditional support. You mean more to me than you'll ever know. 



\begin{abstract}
Liquid argon (LAr) time projection chambers (TPC) have rapidly increased in importance as particle detectors throughout the past four decades. While much research has been completed, there are still many areas which require further development to build and operate the next generation LAr TPC experiment, such as the Deep Underground Neutrino Experiment (DUNE). These include high voltage breakdown, argon purification and purity monitoring, and vacuum ultraviolet (VUV) scintillation light measurement.

Visual monitoring of high voltage breakdown is helpful in allowing assessment of the performance of high voltage component design. Thus, a system of cryogenic cameras, the first of its kind, was developed for use in a large LAr cryostat, without the need for additional electronics heating. The system functioned without problem for 50 days at cryogenic temperature, with some degradation of image quality, and provided a useful monitor for the DUNE 35-ton cryogenics systems. The system did not observe any high voltage breakdowns during the run. Further development of the concept is ongoing for future installation in other experiments.

The monitoring of LAr purity using TPC data is a fundamental study for LAr TPC experiments. However, the study has not been performed for a large LAr TPC in the presence of high electronic noise. Custom software was developed and validated for the accurate reconstruction of signals in noisy TPC data. The results of the reconstruction were used to successfully measure the LAr electron lifetime with an uncertainty comparable to alternate methods of measurement. The electron lifetime of the 35-ton Phase II run is determined to be $4.12 \pm 0.17$ (stat.) \pm 0.40 (syst.) ms.

For general purpose research and development of high purity LAr as a particle detection medium, a dedicated test stand was designed, constructed, and commissioned. The system is used to test the gaseous photomultiplier (GPM) performance at cryogenic temperatures. The GPM functions with photoelectron multiplication at $77 \mathrm{~K}$, at a reduced gain. Further study is required to show the detector's direct sensitivity to LAr VUV scintillation light.
\end{abstract}





\section{Table of contents}

$\begin{array}{ll}\text { List of figures } & \text { xv }\end{array}$

$\begin{array}{ll}\text { List of tables } & \text { Xxv }\end{array}$

$\begin{array}{ll}\text { Nomenclature } & \text { xxvii }\end{array}$

1 Introduction and Theory 1

1.1 Introduction . . . . . . . . . . . . . . . . . . 2

1.2 Brief History of Neutrino Oscillations . . . . . . . . . . . . 2

1.3 Theory of Neutrino Oscillations _................ . . 3

1.3.1 Remaining Unanswered Questions . . . . . . . . . . . . . . . 7

1.4 Energy Loss In Matter . . . . . . . . . . . . . . . . . . . . . 9

1.4.1 Charged Particle Energy Loss . . . . . . . . . . . . . . . 10

1.4.2 Photon Interactions in Matter . . . . . . . . . . . . . . . . . 11

1.4.3 Other Forms of Particle Interactions with Matter . . . . . . . . . 14

1.5 Neutrino Interactions with Matter . . . . . . . . . . . . . . 15

1.6 Summary . . . . . . . . . . . . . . . . . . 17

2 Liquid Argon Time Projection Chambers 19

2.1 LAr TPC Description and Operating Principle . . . . . . . . . . . . . . . 19

2.1 .1 Dual Phase LAr TPC . . . . . . . . . . . . . . . . . . 21

2.1 .2 Signal Generation . . . . . . . . . . . . . . . . . . 22

2.2 Physics of LAr Detectors . . . . . . . . . . . . . . . . . . . . . . . 22

2.2 .1 Ionisation and Scintillation . . . . . . . . . . . . . . 25

2.2.2 Ionisation Electron Drift . . . . . . . . . . . . . . . . 26

2.2.3 Free Electron Attachment to Impurities . . . . . . . . . . . . . . 26

2.2 .4 Positive Space Charge . . . . . . . . . . . . . . . . . . . . . . . . . . . . . . . . . . . . . 39

2.2 .5 Electron Diffusion _. . . . . . . . . . . . . . . 30

2.2 .6 Recombination . . . . . . . . . . . . . 33 
2.2.7 Energy Resolution . . . . . . . . . . . . . . . . . 35

2.2.8 Background Processes . . . . . . . . . . . . . . . . . . 35

2.2 .9 Particle Detection . . . . . . . . . . . . . . . . . . 37

2.3 Past, Current, and Future LAr TPCs and Their Applications . . . . . . . . . 38

2.3.1 Past LAr TPCs . . . . . . . . . . . . . . . . . . 38

2.3.2 Current and Future LAr TPCs . . . . . . . . . . . . . . . . 41

2.3.3 The Deep Underground Neutrino Experiment . . . . . . . . . . . 43

2.3.4 Dual Phase Argon TPCs . . . . . . . . . . . . . . . . . . . . . 48

2.4 Summary . . . . . . . . . . . . . . . . . . 50

3 The DUNE 35-ton Prototype Detector 51

3.1 The 35 -ton Experiment . . . . . . . . . . . . . . . . . . . 51

3.235 -ton Detector Description $\ldots \ldots \ldots \ldots 2$

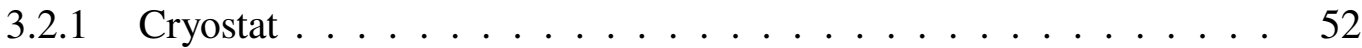

3.2.2 LAr Purification and Monitoring . . . . . . . . . . . . . . 53

3.2 .3 ТPC . . . . . . . . . . . . . . . . . . 54

3.2 .4 TPC Data Acquisition . . . . . . . . . . . . . 56

3.2 .5 Muon Detectors . . . . . . . . . . . . . . . . . . . 58

3.2 .6 Photon Detectors . . . . . . . . . . . . . . . 60

3.3 Phase I . . . . . . . . . . . . . . . . . . . . . 60

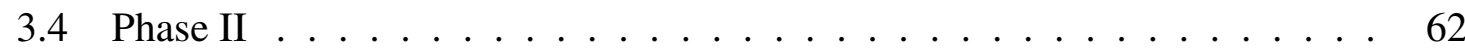

3.4 .1 Noise . . . . . . . . . . . . . . . 63

3.4 .2 Stuck Bits . . . . . . . . . . . . . . . . . . 64

3.4 .3 Purity ............................. 64

3.5 Calibrating Muon Counter and TPC Coordinates _ . . . . . . . . . . 65

3.6 Summary . . . . . . . . . . . . . . . . . . . 68

4 Cameras In The 35-ton Cryostat

4.1 Motivation . . . . . . . . . . . . . . . . . 71

4.2 Camera Selection . . . . . . . . . . . . . . . . . 72

4.3 Camera System Description . . . . . . . . . . . . . . . . . . 74

4.3 .1 Camera Modules . . . . . . . . . . . . . . . . 76

4.3.2 Mounting Bracket . . . . . . . . . . . . . 78

4.3 .3 Cables . . . . . . . . . . . . . . . . 78

4.3 .4 Power Supply . . . . . . . . . . . . . . . . . . . 78

4.3.5 PT100 Conditioner . . . . . . . . . . . . . . 78

4.3.6 Data Acquisition . . . . . . . . . . . . . . . . 79 
4.4 Camera Performance Studies . . . . . . . . . . . . . . . . . . . . . 82

4.5 System Operation and Results _ . . . . . . . . . . . . . . . . . . 86

4.6 Summary . . . . . . . . . . . . . . . . . . . . . . . 89

5 Simulation and Reconstruction of 35-ton Data 91

$5.1 \quad 35$-ton Simulation . . . . . . . . . . . . . . . . . . . . 91

$5.1 .1 \quad$ LArSoft . . . . . . . . . . . . . . . . . . . . 92

5.2 Signal Processing . . . . . . . . . . . . . . . . . . . . 96

5.2.1 Bad Channels . . . . . . . . . . . . . . . . . . . 96

5.2.2 Pedestal Subtraction . . . . . . . . . . . . . . . . . 97

5.2 .3 Stuck Bit Mitigation _ . . . . . . . . . . . . . . . . . . . 98

5.2.4 Coherent Noise Subtraction _. . . . . . . . . . . . . . . . 98

5.2 .5 Deconvolution . . . . . . . . . . . . . . . . 98

5.3 Hit Reconstruction . . . . . . . . . . . . . . . . . . . . . . . . 102

5.3.1 Standard Hit Reconstruction . . . . . . . . . . . . . . . . 102

5.3.2 Robust Hit Reconstruction . . . . . . . . . . . . . . . . . 103

5.3 .3 Track Finding . . . . . . . . . . . . . . . . . . . . 110

5.3.4 Track Hit Backtracker . . . . . . . . . . . . . . . . . 116

5.4 Event Reconstruction . . . . . . . . . . . . . . . . . . . . . . 117

5.5 Summary . . . . . . . . . . . . . . . . . . 117

6 Analysis of 35-ton Electron Lifetime 119

6.1 Electron Lifetime Analysis . . . . . . . . . . . . . . . . . . . . . . . 119

6.1 .1 Method ...................... 120

6.1.2 Landau Gaussian Convolution Fits . . . . . . . . . . . . . . . 121

$6.1 .3 \quad 35$-ton Data Set . . . . . . . . . . . . . . . . . . 123

6.1.4 Preliminary Exponential Decay Lifetime . . . . . . . . . . . . . 124

6.2 Simulation Reconstruction Performance . . . . . . . . . . . . . . . . . . 128

6.2.1 Simulated Samples . . . . . . . . . . . . . . . . . . . . . . . . . . 129

6.2.2 Track Reconstruction Efficiency . . . . . . . . . . . . . . . . . . 129

6.2.3 Hit Reconstruction Efficiency and Purity . . . . . . . . . . . . 130

6.2.4 Charge Resolution and Accuracy . . . . . . . . . . . . . . . . . 137

6.3 35-ton Data Reconstruction Performance . . . . . . . . . . . . . . . . . . 143

6.3.1 Track Reconstruction Efficiency . . . . . . . . . . . . . . . . 143

6.3.2 Hit Reconstruction Efficiency . . . . . . . . . . . . . . . . . 143

6.4 Comparing Data With Simulation . . . . . . . . . . . . . . . 147

6.4.1 "Assumed" hits in data and simulation . . . . . . . . . . . . . . . 147 
6.4.2 Reducing bias by correcting signal scale factors . . . . . . . . . . 147

6.4.3 Data, Simulation, and MC Truth hit charge . . . . . . . . . . . 149

6.4.4 Impact of hit finding threshold . . . . . . . . . . . . . 152

6.4.5 Reducing bias by applying fiducial cuts . . . . . . . . . . . . 152

6.5 Correcting for Electron Lifetime Bias _ . . . . . . . . . . . . . . . . 153

6.5.1 Systematic Uncertainties . . . . . . . . . . . . . . . . 156

6.6 Summary . . . . . . . . . . . . . . . . . . . 157

7 The Sheffield LAr Test Stand 159

7.1 Design and Construction . . . . . . . . . . . . . . . 160

7.1.1 Cryogenics and Piping . . . . . . . . . . . . . . . . . 160

7.1 .2 Instrumentation . . . . . . . . . . . . . . . . . 167

7.1 .3 Argon Purifier . . . . . . . . . . . . . . . . 169

7.1 .4 Slow Control . . . . . . . . . . . . . . . . . . . . 170

7.1.5 Electronics Feedthrough and Cables . . . . . . . . . . . . 171

7.2 Operation . . . . . . . . . . . . . . . . . 171

7.3 Summary . . . . . . . . . . . . . . . . . . . 173

8 Cryogenic Gaseous Photomultiplier Development 175

8.1 Micro-Patterned Gaseous Detectors . . . . . . . . . . . . . . . 176

8.1.1 Photocathodes . . . . . . . . . . . . . . 178

8.2 Detector Description . . . . . . . . . . . . . . . . . . . . 180

8.3 Operating Principle . . . . . . . . . . . . . . . . . . 180

8.4 Data Acquisition . . . . . . . . . . . . . . . . . . . . 182

8.4.1 Front End Electronics _ . . . . . . . . . . . . . . . . 182

8.4.2 System Control and Analysis . . . . . . . . . . . . . . . . . . 185

8.5 Detector Characterisation . . . . . . . . . . . . . . . . . . 188

8.5.1 Experiment 1: Room Temperature Functionality . . . . . . . . . 189

8.5.2 Experiment 2: Warm/Cold Gain Comparison . . . . . . . . . . . 191

8.5.3 Experiment 3: Argon Scintillation Light Measurement . . . . . . . 193

8.5.4 Experiment 4: 77 K Operation . . . . . . . . . . . . . . . . 200

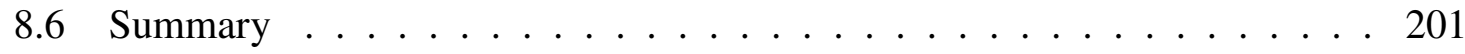

9 Conclusion $\quad 205$

$\begin{array}{ll}\text { References } & 207\end{array}$ 


\section{List of figures}

1.1 Two possible orderings of the neutrino mass squared differences. Left: NO, right: IO. . . . . . . . . . . . . . . . .

1.2 Energy loss in copper as a function of electron energy. For low energies, ionisation processes dominate, whereas for larger energies, radiative effects dominate. . . . . . . . . . . . . . . . . . .

1.3 Probability distribution of energy loss $\Delta$ from two theories (Landau-Vavilov and Bethe-Fano). Mean energy loss, $\langle\Delta\rangle$, and most probable energy loss, $\Delta_{p}$, are indicated. $M_{0}(\Delta)$ and $M_{1}(\Delta)$ are the cumulative 0th moment (mean number of collisions) and cumulative 1st moment (mean energy loss) in crossing the detector. . . . . . . . . . . . . . . . . 12

1.4 Photon total cross-sections as a function of energy in carbon and lead. . . . 13

2.1 Schematic of the elements and operation of a LAr TPC . . . . . . . . . . . 21

2.2 Illustration of the ArDM two-phase argon TPC . . . . . . . . . . . . 23

2.3 Weighting field on a TPC induction wire (contours), and electric field lines when fully biased (yellow lines). . . . . . . . . . . . . . . . . 23

2.4 Top: measured electron drift velocities. Bottom: electron mobility, shown with global fit (red band). Left: gaseous argon. Right: LAr. Measurements are shown for different drift distances. Top horizontal axis: applied electric field. Bottom horizontal axis: electric field divided by number density of argon atoms in units of $T d=10^{-17} \mathrm{~V} \mathrm{~cm}^{2}$. The expected mobility for zero field is shown as $518 \mathrm{~cm}^{2} / \mathrm{V} / \mathrm{s}$. . . . . . . . . . . . . . . . 27

2.5 Electron attachment rate constant in $\mathrm{LAr}_{\mathrm{S}} \mathrm{SF}_{6}(\triangle), \mathrm{N}_{2} \mathrm{O}(\square)$, and $\mathrm{O}_{2}(\circ)$. . 30

2.6 Space charge distorted electric field at the anode (Ea/E0) and cathode (Ec/E0),

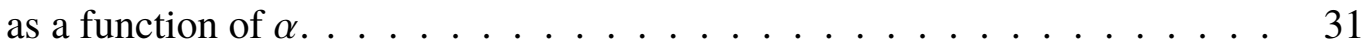

2.7 Space charge distorted field at the anode for the DUNE 35-ton TPC. . . . . 31

2.8 Illustration of the diffusion process starting from a point source and drifting across a distance $d$ in an electric field $E$. . . . . . . . . . . . . . . . 32 
2.9 Global fit on electron energy, $\epsilon_{L}$, for LAr. . . . . . . . . . . . . . . . . 34

2.10 Comparison of best fit recombination coefficients for three models using the same underlying data for each (data not shown). . . . . . . . . . . . 36

2.11 Example event display showing ionising muon track with several delta electron tracks emanating from the primary particle, from two different views. 38

2.12 Photo of a slice of the ICARUS detector showing anode wire planes (left), central cathode (right), field shaping rings, and VUV sensitive PMT. . . . . 40

2.13 A charged current $v_{\mu}$ event candidate from the ArgoNeuT LAr TPC. . . . . 42

2.14 Schematic of the MicroBooNE cryostat and TPC, showing major elements including field shaping rings, PMT mounting rack (left side, frame with horizontal bars), and anode wiring frame (left side of cryostat, frame supported by "X" cross beams). . . . . . . . . . . . . . . . . . . .

2.15 Illustration of the DUNE underground complex, including four $10 \mathrm{kt}$ detector modules, and central service hall for cryogenics and electronics. . . . . . . 44

2.16 The main DUNE detector components and schematic. . . . . . . . . . . . . 44

2.17 Schematic of DUNE reference TPC design, showing APAs (red), CPAs (blue), and field shaping cage (green/yellow). . . . . . . . . . . . . 46

2.18 Schematic of charge readout in the WA105 TPC . . . . . . . . . . . . 49

2.19 Diagram of low capacitance pixel-based charge readout anode. . . . . . . . 49

3.1 Cutaway illustration of the 35-ton cryostat dimensions and construction details. 52

3.2 Diagram of 35-ton TPC inside cryostat. . . . . . . . . . . . . 53

3.3 Diagram of an ICARUS style purity monitor. . . . . . . . . . . . . . . 55

3.4 Photo of a 35-ton purity monitor. . . . . . . . . . . . . . . 55

3.5 TPC coordinate and numbering system. . . . . . . . . . . . 57

3.6 Diagram of wire planes on APAs in the 35-ton Prototype TPC. . . . . . . . 57

3.7 Muon counters location and numbering with respect to the 35-ton cryostat and TPC, with the detector coordinate system. . . . . . . . . . . . . 59

3.8 Locations and numbering scheme for the PDs in the 35-ton TPC. . . . . . . 61

3.9 35-ton Phase I electron lifetime as measured by the PrMs. . . . . . . . . . . 62

3.10 The Fourier transform of the ADC values on all channels in the LNS. . . . . 64

3.11 Measured electron lifetime by PrM 2 over the course of the Phase II run. The shaded region represents the times when the analysed data were taken. . . . 66

3.12 Number of intersections of muon counter and reconstructed track for a given grid search offset applied in $x$ and $z \ldots \ldots \ldots \ldots \ldots$ 
3.13 Measured $z$ position of North-South muon counters by finding the wire with largest standardised ADC value for each TDC tick. Distributions are fit to Gaussian to obtain central value. Dashed lines indicate independently measured $z$ boundaries of the corresponding muon counter pairs. . . . . .

4.1 Measured electric breakdown fields compiled from many studies. . . . . . . 73

4.2 OEM camera \#1 (a) at room temperature and (b) submerged in $\mathrm{LN}_{2}$. . . . . 74

4.3 OEM camera \#2 (a) at room temperature and (b) submerged in $\mathrm{LN}_{2}$. . . . . 75

4.4 OEM camera \#3 (a) at room temperature and (b) submerged in $\mathrm{LN}_{2}$. . . . 75

4.5 Sony XAR800C (a) at room temperature and (b) submerged in $\mathrm{LN}_{2}$. . . . . 75

4.6 Camera system block diagram including specific cable gauge and connector information. . . . . . . . . . . . . . 76

4.7 Exploded diagram of camera and components in housing. From left to right: Conflat-40 flange with 9-pin D-subminiature feedthrough, double sided barrel connector, PT100 temperature sensor (green wires), camera centred (red, yellow, black wires), heating resistors (blue wires), flange with glass window. 77

4.8 Left: a complete, sealed camera module. Right: camera innards, pulled apart for internal component identification. . . . . . . . . . . . . . 77

4.9 Mounting bracket components description. . . . . . . . . . . . . . . 79

4.10 Custom $10 \mathrm{~V}$ and $12 \mathrm{~V}$ power supply unit, 10 output channels for each voltage. Each channel was individually controllable using a standard digital TTL pulse. 80

4.11 Custom PT100 signal conditioning unit, 10 channels capability. Temperature is read out on a proportional voltage scale. . . . . . . . . . . .

4.12 Measurement of camera self heating from power on in LAr. Local temperature of the camera inside the housing, on the outside of the glass window, and in the LAr away from the camera housing. . . . . . . . . . . . . . .

4.13 Measurement of camera returning to ambient temperature after power switch off in LAr. Local temperature of the camera inside the housing, on the outside of the glass window, and in the LAr away from the camera housing. . . . .

4.14 Local temperatures in and around the camera housing following switch on of the internal heater in LAr. The line representing the "Glass" temperature is hidden behind the "Control" line. . . . . . . . . . . . . . . . .

4.15 Camera stills taken in warm (left) and cold (right) at a distance of $10 \mathrm{~cm}$ from the light source, and with light spot spacings of $7 \mathrm{~mm}, 4 \mathrm{~mm}, 2 \mathrm{~mm}$, and $1 \mathrm{~mm} .85$

4.16 (a) Photo of spark and equipment taken with normal digital camera. The cryogenic camera can be seen at the bottom of the image. (b) Photo of spark taken by cryogenic camera while submerged in $\mathrm{LN}_{2} \ldots \ldots \ldots$. . . . . . 
4.17 RGB histograms of images taken before (top) and after (bottom) loss of colour encoding due to cooling of the camera to cryogenic temperature. . .

4.18 Calibration images for system cameras. Upper (left to right): Phase separator, ullage, cathode top right, bottom right. Lower (left to right): Cooldown sprayers, cathode top left, bottom left, HV feedthrough. Top four images taken with halogen light illumination. Bottom four images taken with LED illumination. Images are left-right inverted. . . . . . . . . . .

4.19 Camera 1 (top) and camera 4 (bottom) images taken at different times. From left to right: before cooldown, immediately after cooldown, after 50 days of cold operation.

5.1 35-ton detector geometry visualisation including cryostat, modular TPC, and muon counters.

5.2 Example of stuck bit mitigation performance. . . . . . . . . . . . . . . 99

5.3 Example of coherent noise subtraction performance. . . . . . . . . . . . . 100

5.4 Simulated field response of three wire planes. . . . . . . . . . . . . . 100

5.5 Theoretical electronics response curves from delta pulse input at time 0 .

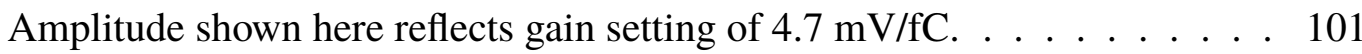

5.6 Event displays showing time on the vertical, and wire number on the horizontal.

(a) Signals on the wire are shown after deconvolution and frequency filtering.

(b) Black dots represent reconstructed hits from the GHF. . . . . . . . . . 104

5.7 Flow diagram of the RHF algorithm . . . . . . . . . . . . . . 105

5.8 Profile histograms of reconstructed hit integral vs. true hit charge for two algorithms.

5.9 Reconstructed drift coordinate versus hit time. The slope of the line represents the drift velocity in the particular region of the APA frame. . . . . . . . . . 110

5.10 Example event with 246 hits in the TPC, plotted at their detector $x$ and $z$ coordinates. Also shown in green is the region of acceptance for this event given the triggered muon counters.

5.11 Example iteration of the MLESAC algorithm applied to the event in Figure 5.10. Given the initial starting randomly sampled points (red points), the best-fit model (red line) does not represent a good fit to the actual physical event, indicated by a high sum of squared residuals (S.S.R.) per degree of freedom (N.D.F.) . . . . . . . . . . . . . . . . . . . . 113 
5.12 Example iteration of the MLESAC algorithm applied to the event in Figure 5.10. Given the initial starting randomly sampled points (red points), the best-fit model (red line) represents a good fit, indicated by a low sum of squared residuals (S.S.R.) per degree of freedom (N.D.F.). . . . . . . . . . 114

6.1 Illustration of the variables which define $\gamma$ (Equation 6.3) $\ldots \ldots \ldots$

6.2 Distributions of hit $d Q / d x$ for "found" and "assumed" hits across all drift times. . . . . . . . . . . . . . . . . . . 125

6.3 Blue points: total number of hits included in each time bin over the full drift window. Black points: fraction of all hits which are "assumed". . . . . . . 125

6.4 Four (of 22 total) bins of $d Q / d x$ spanning different regions of the drift window. (a) and (d) correspond to hits originally near the anode and cathode, respectively. Landau $\otimes$ Gauss fits are shown as red curves, with best fit parameters included. . . . . . . . . . . . . . . 126

$6.5 \chi^{2} / \mathrm{NDF}$ of Landau $\otimes$ Gauss best fit curve to data. . . . . . . . . . . . . . . 127

6.6 Most probable hit $d Q / d x$ measured at the anode as a function of drift time, plotted on log scale. Exponential fit to fiducial range shown in red. . . . . . 127

6.7 Track finding efficiency (black text) and associated upper and lower binomial errors (red text) for all possible East-West muon counter coincidences in the simulated data set (3.5 ms electron lifetime, $1.0 \mu$ parameter). . . . . . . . .

6.8 Efficiency of hit finding as a function of $\mu$ for muon counter triggers 9 and 31 (44 $\pm 15 \mathrm{~cm}$ drift distance) and several values of $\tau_{\mathrm{MC}} \ldots \ldots . . . \ldots 133$

6.9 Purity of hit finding as a function of $\mu$ for muon counter triggers 9 and 31 (44 $\pm 15 \mathrm{~cm}$ drift distance) and several values of $\tau_{\mathrm{MC}} \ldots \ldots$. . . . . . . . . . 134

6.10 Efficiency of hit finding in the simulation as a function of drift distance for $\mu=1.2$ and several values of $\tau_{\mathrm{MC}} \ldots \ldots \ldots 134$

6.11 Efficiency of hit finding in the simulation as a function of drift distance for $\mu=1.2$ and several values of $\tau_{\mathrm{MC}} \ldots \ldots \ldots \ldots$

6.12 Hit finding efficiency (black text) and associated uncertainty (red text) for events of all possible combinations of East-West counter coincidences $(3.5 \mathrm{~ms}$ simulated lifetime and $1.0 \mu$ parameter) . . . . . . . . . . . . 136

6.13 Relative locations of East-West muon counters and the detector. . . . . . . 136

6.14 Hit finding purity (black text) and associated uncertainty (red text) for events with all possible combinations of East-West counter coincidences (3.5 ms simulated lifetime and $1.0 \mu$ parameter $) \ldots \ldots . \ldots . \ldots 138$ 
6.15 Hit finding efficiency versus MCT hit charge for several values of $\mu$. The histogram (arbitrary normalisation) of $Q_{\mathrm{MC}}$ is shown for comparison on the same horizontal scale. . . . . . . . . . . . . . . . . . . . . 139

6.16 Profile histogram of reconstructed hit charge given associated MCT hit charge. Error bars represent the standard error on the mean, the shaded region represents the standard deviation. . . . . . . . . . . . . . . . . 140

6.17 Histogram of charge residuals, $R_{q}$, for several values of $\mu$. . . . . . . . . 140

6.18 Mean charge residual $R_{q}$ versus $Q_{\mathrm{MC}}$ for several values of $\mu$. . . . . . . 141

6.19 Charge resolution vs. hit charge for several values of $\mu$. The histogram (arbitrary normalisation) of MC hit charge is shown in shaded grey for comparison. . . . . . . . . . . . . . . . . . . . . 142

6.20 Track finding efficiency (black text) and associated upper and lower binomial errors (red text) for all possible East-West muon counter coincidences in the full 35-ton data set. . . . . . . . . . . . . . . . . . . . . . . . . . 144

6.21 Cartoon diagram of typical East-West muon event in the 35-ton detector. In this view, wire numbers (edges labelled) increase from right to left. . . . . . 146

6.22 Number of hits on each wire in each long-drift TPC. Red regions denote bad wires. . . . . . . . . . . . . . . . . . . . . 148

6.23 Hit finding efficiency on each wire for each of the long-drift TPCs. Red regions denote bad wires. . . . . . . . . . . . . . . . . . . . 148

6.24 "Assumed" hit fraction for data and simulation for every collection plane wire. The dashed line shows equal "assumed" hit fraction in data and simulation.

6.25 Exponential fit $(d Q / d x)_{0}$ parameter for all simulated datasets. Vertical blue line represents the true data $(d Q / d x)_{0}$ value, and the true $\mu$ parameter is determined through interpolation. . . . . . . . . . . . . . . . . 150

6.26 Data, simulation, and MCT $d Q / d x$ histograms for (a) short, (b) short-middle, (c) middle-long, and (d) long drift distances. Simulated samples shown use an electron lifetime of $4 \mathrm{~ms}$.

6.27 Hypothetical effect of variable hit finding threshold (orange) on Landau (blue) MPV measurement. The dashed curve represents the parts of the Landau which are cut off by the threshold.

6.28 Measured electron lifetime of simulated samples and 35-ton data for different values of $m=\mu / 1.153$.

6.29 Data $d Q / d x$ MPV (black) overlaid with most similar simulated $d Q / d x$ MPV (blue). Green band represents the uncertainty due to $\mu$. . . . . . . . . . . . 154

7.1 Schematic of Sheffield LAr test stand and main operational components. . . 161 
7.2 Image of assembled LAr test stand and components. . . . . . . . . . . . . 162

7.3 Outside of main LAr Dewar during filling. . . . . . . . . . . . . . . . . . 163

7.4 Purifier vessel is shown as the vertically oriented cylinder with coiled heating cable wrapped around it. Also in the image, dew point sensor for monitoring the activation process (rectangular block of steel with instrument on top, right side of the image centred vertically) . . . . . . . . . . . . . . . 165

7.5 Top of recondenser vessel (covered in blue and black insulation). Also shown: capacitive level meter and display (CryoGauge 242), solenoid valve (brass valve with grey switching element), PT100 temperature sensor (small element mounted on the near side of the vessel with white wire), and presser transducer (instrument on end of pipe which is bent at 2 o'clock, right side of image). Spiralled pipe on the left ensures the cold boiloff gas warms sufficiently before reaching the back pressure regulator, which can freeze. . 166

7.6 Phase diagram of argon with nitrogen boiling line superposed. . . . . . . . 168

7.7 Slow control logic feedback loops for test stand subsystems. . . . . . . . . . 171

7.8 Process measurements for the first engineering cooldown test. The stable LAr and $\mathrm{LN}_{2}$ system thermodynamics was demonstrated for three hours before warm up. Hour 0 - Argon fill begins. Hour 1 - Nitrogen fill begins. Hours 1-3 - Normal operation. Hour 4 - Test ended, warm-up of the system begins. 174

8.1 Photo of typical THGEM electrode. . . . . . . . . . . . . . . . . . . 177

8.2 Closeup photos of THCOBRA top strips (right) and anode/cathode strips (left).179

8.3 Quantum efficiency of semi-transparent (S.T.) and reflective photocathodes of CsI and $\mathrm{KBr}$ versus light wavelength. . . . . . . . . . . . . . . . . . . 179

8.4 Photo of GPM front face. Through the $\mathrm{MgF}_{2}$ window can be seen the extraction grid and the top of the first THGEM which is coated in CsI (green).181

8.5 Photo of GPM rear face, showing 12-pin HV CF-40 flange with signal connections, and two gas flow pipes. . . . . . . . . . . . . . . 181

8.6 Diagram of GPM operation. . . . . . . . . . . . . . . . . . . . . 182

8.7 Representative GPM pulses following preamplification but before Gaussian shaping. Two anode strip signals are shown (blue, green), and two top strip signals are shown $($ red, black). . . . . . . . . . . . . . . . . . . 183

8.8 Block diagram of front end electronics and interfaces between system components. . . . . . . . . . . . . . . . . . . . . . . . 184 
8.9 Four channel CSP readout board showing two channels in use. Base circuit board was designed and manufactured at the Universidade de Aveiro. Circuit is installed in NIM module for electromagnetic shielding and stable DC power supply. . . . . . . . . . . . . . . . . . . . . . . . . 184

8.10 Four Cremat CR-160 evaluation boards with which to read out CR-200 shaping amplifiers, which have optional coarse and fine gain manual controls. Boards are installed in double-wide NIM module for electromagnetic shielding and stable DC power supply. . . . . . . . . . . . . . . . . . . . . . . . . 184

8.11 Calibration of Cremat CR-111 premplifiers, AC coupled with $10 \mathrm{nF}$ capacitor, and Cremat CR-200-8 $\mu$ s shaping amplifier. Horizontal axis is calculated as $10 \mathrm{nF}$ multiplied by the input step voltage. . . . . . . . . . . . . . . 186

8.12 Left to right: 155 bar supply cylinder of $\mathrm{CH}_{4}(5 \%) / \mathrm{Ne}$, mass flow controller, GPM inlet, GPM outlet, small flask of oil. The outlet gas is vented to atmosphere through the production of bubbles in the oil (see Figure 8.13).

8.13 Photo of the small flask filled with oil in which the GPM gas is vented. Two small bubbles can be seen floating to the oil surface indicating that gas is flowing through the GPM.

8.14 Format of example binary data file with 2 channels of data and multiple waveform acquisitions. . . . . . . . . . . . . . . . . 187

8.15 Photo of GPM during room temperature test of functionality. . . . . . . . 190

8.16 Experiment 2 setup of GPM and LED in insulated bucket, submerged in dry ice chips. . . . . . . . . . . . . . . . . . . . . . . . 192

8.17 Pulse height distribution for anode (left) and top (right) strips. Room temperature operation, with operating voltages as in Table 8.2 with $\Delta V=-10$ V.194

8.18 GPM absolute gain measurements at room temperature and in dry ice. $\Delta V$ represents the voltages added to the THGEM and THCOBRA components, in addition to the default values in Table 8.2 . . . . . . . . . . . . . . . 194

8.19 GPM (left) and PMT (right) experiment setup in the test stand before the Dewar was closed. Detectors were supported by PTFE mount which allows the sensitive areas of the detectors to be exposed to the same experiment volume. White sheet of PTFE is also shown inside the Dewar, roughly coated with TPB

8.20 PMT+TPB in nitrogen gas. Pulse amplitude distribution scaled by total run time (2040 seconds) to acquire $10 \mathrm{k}$ pulses. . . . . . . . . . . . . .

8.21 PMT+TPB in argon gas. Pulse amplitude distribution scaled by total run time (596 seconds) to acquire 10k pulses. 
8.22 Right: Argon scintillation raw PMT pulse. Left: Argon scintillation PMT pulse after shaping. . . . . . . . . . . . . . . . . . . . 198

8.23 Pulse amplitude distribution of GPM in nitrogen gas at room temperature. Manual coarse gain (10x) on Cremat shaping amplifier switched off. . . . . 198

8.24 Pulse amplitude distribution of GPM in argon gas at room temperature. Manual coarse gain (10x) on Cremat shaping amplifier switched on. . . . . 198

8.25 Distribution of time between triggers in the GPM while in nitrogen gas. The lifetime of the exponential is related to the average $\Delta T \ldots \ldots$. . . . . 199

8.26 Distribution of time between triggers in the GPM while in argon gas. The lifetime of the exponential is related to the average $\Delta T \ldots \ldots 200$

8.27 Single photoelectron pulse height distribution of GPM in $\mathrm{LN}_{2}$ and $\Delta V=$ +100 V. No LED. . . . . . . . . . . . . . . . . . . . . . 202

8.28 Single photoelectron pulse height distribution of GPM in $\mathrm{LN}_{2}$ and $\Delta V=$ +100 V. With $355 \mathrm{~nm}$ LED light. . . . . . . . . . . . . . . . . . . 202 



\section{List of tables}

1.1 Current neutrino oscillation global best fit parameter values and $3 \sigma$ ranges. $\quad 8$

2.1 Physical properties of LAr. . . . . . . . . . . . . . . . . . . . . 24

2.2 Summary of performance parameters for DUNE far detector. . . . . . . . . 47

3.1 Measured $z$ offset using $x$-independent method. *Note that counters $0,5,16$, $21,22,27,38$, and 43 straddle the edges of the TPC and are not reliable in measuring the $z$ offset. . . . . . . . . . . . . . . 67

5.1 Accurate $(\boldsymbol{})$ or inaccurate $(\boldsymbol{X})$ simulated output waveform when ADCs of each type are added from both sources. . . . . . . . . . . . . . . . 95

6.1 Number of data acquisition runs, triggered events, and both types of hits from the full initial data sample, following each subsequent data quality/acceptance cut. . . . . . . . . . . . . . . . . . . . . 123

6.2 Truth table for comparing the existence of MCT and reconstructed hits. . . 129

6.3 Examples of reconstructed events which cross the horizontal gap between TPCs 3 and 5. "|" = Edge wire, " _" = Bad wire, "X" = Hit found on wire, "." $=$ Hit not found on wire . . . . . . . . . . . . . . . . 146

8.1 Left: Default voltages applied to GPM components, relative to ground. Right: Effective component voltages. . . . . . . . . . . . . . . . . . . 190

8.2 GPM HV scanning scheme for gain measurements. . . . . . . . . . . . . 192 



\title{
Nomenclature
}

\author{
Greek Symbols
}

$0 v \beta \beta \quad$ Neutrinoless double beta

\section{Acronyms / Abbreviations}

$\begin{array}{ll}\text { ADC } & \text { Analogue-to-Digital Conversion } \\ \text { ADC } & \text { Analogue-to-Digital Converter } \\ \text { APA } & \text { Anode Plane Assembly } \\ \text { API } & \text { Application Programming Interface } \\ \text { ASIC } & \text { Application-Specific Integrated Circuit } \\ \text { BNB } & \text { Booster Neutrino Beam } \\ \text { CC } & \text { Charged Current } \\ \text { CCD } & \text { Charge Coupled Device } \\ \text { CERN } & \text { Conseil Européen pour la Recherche Nucléaire } \\ \text { CMOS } & \text { Complementary Metal-Oxide Semiconductor } \\ \text { CNGS } & \text { CERN Neutrinos to Gran Sasso } \\ \text { CPA } & \text { Cathode Plane Assembly } \\ \text { CP } & \text { Charge-Parity symmetry } \\ \text { CPT } & \text { Charge-Parity-Time symmetry } \\ \text { CRY } & \text { Cosmic Ray Shower }\end{array}$




$\begin{array}{ll}\text { CSP } & \text { Charge Sensitive Preamplifier } \\ \text { CTE } & \text { Coefficient of Thermal Expansion }\end{array}$

DAQ Data Acquisition

DIS Deep Inelastic Scattering

DUNE Deep Underground Neutrino Experiment

DVR Digital Video Recorder

ES Elastic Scattering

FEMB Front-end Motherboard

Fermilab Fermi National Accelerator Laboratory

FHiCL Fermilab Hierarchical Configuration Language

FN False Negative

FP False Positive

GHF Gauss Hit Finder

GPM Gaseous Photomultiplier

HNS High-noise State

HV High Voltage

IO Inverted Ordering

LAPD Liquid Argon Purity Demonstrator

LAr Liquid Argon

LArSoft Liquid Argon Software

LEM Large Electron Multiplier

$\mathrm{LN}_{2} \quad$ Liquid Nitrogen

LNS Low-noise State

LXe Liquid Xenon 


\begin{tabular}{|c|c|}
\hline $\mathrm{MC}$ & Monte Carlo \\
\hline MCT & Monte Carlo Truth \\
\hline MIP & Minimally Ionising Particle \\
\hline MLESAC & Maximum Likelihood Estimator Sample Consensus \\
\hline MPV & Most Probable Value \\
\hline MSW & Mikheev-Smirnov-Wolfenstein \\
\hline $\mathrm{NC}$ & Neutral Current \\
\hline $\mathrm{NO}$ & Normal Ordering \\
\hline NuMI & Neutrinos at the Main Injector \\
\hline OEM & Original Equipment Manufacturer \\
\hline PCB & Printed Circuit Board \\
\hline PDF & Probability Density Function \\
\hline PD & Photon Detector \\
\hline PEEK & Polyetheretherketone \\
\hline PET & Positron Emission Tomography \\
\hline PMNS & Pontecorvo-Maki-Nakagawa-Sakata \\
\hline PMT & Photomultiplier Tube \\
\hline $\operatorname{PrM}$ & Purity Monitor \\
\hline PTFE & Polytetrafluoroethylene \\
\hline RHF & Robust Hit Finder \\
\hline RTD & Resistive Temperature Device \\
\hline SBN & Short Baseline Neutrino \\
\hline $\mathrm{SiPM}$ & Silicon Photomultiplier \\
\hline SM & Standard Model \\
\hline
\end{tabular}


S/N Signal-to-Noise Ratio

SSR Solid State Relay

SS Stainless Steel

TDC Time-to-Digital Converter

THB Track Hit Backtracker

THCOBRA Thick COBRA

THGEM Thick Gas Electron Multiplier

TN True Negative

TPB Tetraphenyl Butadiene

TPC Time Projection Chamber

TP True Positive

VUV Vacuum Ultraviolet

WIMP Weakly Interacting Massive Particle 


\section{Chapter 1}

\section{Introduction and Theory}

The "Standard Model" (SM) of particle physics is a very well tested and complete model to describe elementary particles and their interactions. However, when it comes to neutrinos, the SM falls quite short in explaining experimental results. For instance, whereas the SM predicts the neutrinos to be massless, oscillation experiments have unequivocally shown them to have non-zero mass. The neutrino masses and other unanswered questions regarding neutrinos are of considerable scientific interest and motivate the development of next-generation neutrino detection experiments.

New physics detector challenges are presented to investigate the properties of neutrinos to high accuracy, in particular, scaling up the size of liquid argon (LAr) time projection chamber (TPC) detectors to many kilotons in mass. This process introduces many new physics and engineering obstacles, such as mitigating and monitoring high voltage (HV) breakdown in LAr, efficient vacuum ultraviolet (VUV) light collection, maintaining and measuring ultra-high levels of argon purity, minimising electronic noise, and more. Many of these issues are addressed in this thesis as research and development toward the Deep Underground Neutrino Experiment (DUNE) in particular, but in general, noble liquid experiments for rare physics detection.

Chapter 1 summarises the current state of theoretical and experimental knowledge of neutrino oscillations and remaining unanswered questions, motivating the next generation experiments. Also in Chapter 1, the theory of particle interactions with matter is introduced which guides the interpretation of measurable signals in particle detectors. Chapter 2 introduces the TPC operating principles and the relevant physics of particle detection and signal creation. Chapter 3 describes the DUNE 35-ton Prototype detector and the physics run. Chapter 4 describes the cryogenic camera system developed at Sheffield for the DUNE 35-ton Prototype. Chapters 5 and 6 describe the analysis of electron lifetime in the 35-ton TPC and the simulations of the detector. Chapter 7 introduces the Sheffield LAr test stand design 
and construction for general purpose LAr research and development. Chapter 8 presents the cryogenic testing of the gaseous photomultiplier (GPM) for direct VUV light collection.

\subsection{Introduction}

Neutrinos are electrically neutral so are only detected via their secondary particles in interactions. The properties of these secondary particles measured in a neutrino detector shed light on the initial neutrino properties, like the energy and flavour. Moreover, the theory of charged particle energy loss in matter motivates the understanding of the signals produced in a LAr TPC and allows precise calibrations. Thus, details of the background neutrino oscillation processes can be better understood. These theories described in this chapter provide a general motivation for the research and development of LAr TPCs for neutrino detection.

\subsection{Brief History of Neutrino Oscillations}

Since their prediction by Pauli in 1930 [1], and their discovery in 1953 by Reines and Cowan [2], neutrinos have been the subject of intense theoretical and experimental study. The story of neutrino oscillations, however, begins in 1968 with Ray Davis' experiment [3] to test Bahcall's solar radiation model [4]. The experiment consisted of a $380 \mathrm{~m}^{3}$ tank of perchloroethylene in the Homestake mine in South Dakota. Electron neutrinos from the sun were detected via the reaction

$$
v_{e}+{ }^{37} \mathrm{Cl} \longrightarrow e^{-}+{ }^{37} \mathrm{Ar},
$$

and the total number of $v_{e}$ interactions was counted by observing the decay of the resulting

${ }^{37} \mathrm{Ar}$ atoms. The experiment detected only a third of the predicted neutrinos in Bahcall's model. This discrepancy became known as the "solar neutrino problem", and in 1968 , Pontecorvo proposed a solution where the neutrino flavour changes in-flight from the source to the detector [5]. The theory is based on a simple model of mixed quantum states and thus was born neutrino oscillations. His theory was built up and formalised in parallel with the work of Maki, Nakagawa, and Sakata [6], which is summarised below in Section 1.3.

Conclusive experimental proof of neutrino oscillations was not shown until 2002 when the Sudbury Neutrino Observatory (SNO) experiment measured the total solar neutrino flux with flavour-dependent and flavour-independent methods of detection [7]. The experiment, which consisted of about one kiloton of chlorinated heavy water at $2100 \mathrm{~m}$ underground, was sensitive to charged current (CC), neutral current (NC), and elastic scattering (ES) neutrino 
interactions. Importantly, SNO was able to distinguish $v_{e}$ from $v_{\mu}$ and $v_{\tau}$ through the distinct signatures of each type of interaction. In CC reactions, electron neutrinos interact with a deuteron, and through the exchange of a $W^{ \pm}$boson (hence the name "charged" current), releases two protons and an energetic electron, which has a particular detection signature. Since the energies of solar neutrinos are smaller than the masses of the muon or tau lepton, this reaction only occurs with $v_{e}$ s. NC reactions, however, are equally sensitive to all neutrino flavours. In these reactions, the neutrino interacts with a deuteron and, through the exchange of a $Z^{0}$ boson (hence the name "neutral" current), breaks apart the deuterium nucleus releasing the proton and neutron, which has a distinct signature. In an ES reaction, a neutrino transfers some of its kinetic energy to an electron in the detector, which has another distinct detection signature. ES reactions are sensitive to all neutrino flavours, though $v_{e}$ dominates by a large factor. The experiment measured the $\mathrm{NC}+\mathrm{ES}$ flux to be about three times higher than the CC flux, which demonstrated the oscillation of solar $v_{e}$ s to the other flavours. Since $\mathrm{SNO}$, much experimental work has been performed to shed light on the oscillation process and the parameters which describe it [8].

\subsection{Theory of Neutrino Oscillations}

There are three flavours of neutrinos and antineutrinos: $\stackrel{(-)}{v_{e}}, \stackrel{\left(-v_{\mu}\right.}{\mu}$, and $\stackrel{(-)}{v_{\tau}}$. They interact with their namesake charged lepton via the weak force. According to experiment, neutrinos (antineutrinos) are always created with left-handed (right-handed) helicity. If more sensitive experiments show the existence of neutrinos and antineutrinos with opposite helicities, those neutrinos would be considered "sterile" and would not interact with the weak $W^{ \pm}$and $Z^{0}$ bosons [9]. In fact, the existence of right-handed neutrinos would play a role in explaining the origin of neutrino masses and mixing, the mass differences between neutrinos and the charged leptons, and the matter-antimatter asymmetry of the universe [8].

The phrase "neutrino oscillation" means that if a neutrino of a particular flavour $l=e, \mu, \tau$ is created with energy $E$ and at distance $L$ from a detector, the probability of detecting a neutrino of a different flavour $l^{\prime}$ at time $T$ is non-zero. More formally, the neutrino eigenstates of the Hamiltonian are $\left|v_{k}\right\rangle$ with eigenvalues $m_{k}$ for $k=1,2,3$ representing the "mass-states" of the neutrinos. These states are related to the "flavour-states" $\left|v_{l}\right\rangle$ by a unitary matrix:

$$
\left|v_{l}\right\rangle=\sum_{k=1,2,3} U_{l k}\left|v_{k}\right\rangle
$$

where $U_{l k}$ is known as the Pontecorvo-Maki-Nakagawa-Sakata (PMNS) matrix $[6,9,10]$. The PMNS matrix is parameterised with three rotation angles and one charge-parity (CP) 
violation phase:

$$
\begin{aligned}
U & =\left(\begin{array}{ccc}
1 & 0 & 0 \\
0 & c_{23} & s_{23} \\
0 & -s_{23} & c_{23}
\end{array}\right)\left(\begin{array}{ccc}
c_{13} & 0 & s_{13} e^{-i \delta_{C P}} \\
0 & 1 & 0 \\
-s_{13} e^{i \delta_{C P}} & 0 & c_{13}
\end{array}\right)\left(\begin{array}{ccc}
c_{12} & s_{12} & 0 \\
-s_{12} & c_{12} & 0 \\
0 & 0 & 1
\end{array}\right) \\
& =\left(\begin{array}{ccc}
c_{12} c_{13} & s_{12} c_{13} & s_{13} e^{-i \delta_{C P}} \\
-s_{12} c_{23}-c_{12} s_{23} s_{13} e^{i \delta_{C P}} & c_{12} c_{23}-s_{12} s_{23} s_{13} e^{i \delta_{C P}} & s_{23} c_{13} \\
s_{12} s_{23}-c_{12} c_{23} s_{13} e^{i \delta_{C P}} & -c_{12} s_{23}-s_{12} c_{23} s_{13} e^{i \delta_{C P}} & c_{23} c_{13}
\end{array}\right)
\end{aligned}
$$

where $c_{i j}=\cos \theta_{i j}, s_{i j}=\sin \theta_{i j}$, the angles $\theta_{i j}=[0, \pi / 2]$, and $\delta_{C P}=[0,2 \pi]$ is the Dirac $\mathrm{CP}$ violation phase. $\theta_{12}$ is commonly referred to as the solar angle, $\theta_{23}$ the atmospheric angle, and $\theta_{13}$ the reactor angle, named for the primary experimental sector in which the measurement of the parameters is particularly sensitive.

To calculate the probability of a neutrino to oscillate to another flavour, consider a neutrino $\left|v_{l}\right\rangle$ in a pure flavour state, Equation 1.2. This equation can also be inverted to write the mass states in terms of the flavour states,

$$
\left|v_{k}\right\rangle=\sum_{\gamma=e, \mu, \tau} U_{\gamma k}^{*}\left|v_{\gamma}\right\rangle
$$

where a new index, $\gamma$, is chosen to avoid confusion later. The wavefunction evolves according to the time-dependent Schrödinger Equation with plane wave solution:

$$
\left|v_{l}(L, T)\right\rangle=\sum_{k=1,2,3} U_{l k}\left|v_{k}(L, T)\right\rangle=\sum_{k=1,2,3} U_{l k}\left|v_{k}(0,0)\right\rangle e^{-i \varphi_{k}}
$$

where $\varphi_{k}=E_{k} T-p_{k} L, E_{k}$ is the neutrino energy, and $p_{k}$ is the momentum. By substituting Equation 1.4 into Equation 1.5, the flavour state at $(L, T)$ is written in terms of the initial flavour state at $(0,0)$ :

$$
\left|v_{l}(L, T)\right\rangle=\sum_{k} \sum_{\gamma} U_{\gamma k}^{*} e^{-i \varphi_{k}} U_{l k}\left|v_{\gamma}(0,0)\right\rangle
$$


The amplitude for an initial neutrino of flavour $l$ at $(0,0)$ to oscillate to a neutrino of flavour $l^{\prime}$ at $(L, T)$ is

$$
\begin{aligned}
A\left(v_{l}(0,0) \rightarrow v_{l^{\prime}}(L, T)\right) & =\left\langle v_{l^{\prime}}(L, T) \mid v_{l}(0,0)\right\rangle \\
& =\sum_{\gamma} \sum_{k} U_{\gamma k} e^{i \varphi_{k}} U_{l^{\prime} k}^{*}\left\langle v_{\gamma}(0,0) \mid v_{l}(0,0)\right\rangle \\
& =\sum_{k} U_{l k} e^{i \varphi_{k}} U_{l^{\prime} k}^{*}
\end{aligned}
$$

where the orthogonality of flavour states ensures $\left\langle v_{\gamma}(0,0) \mid v_{l}(0,0)\right\rangle=\delta_{\gamma l}$. The probability of this oscillation is the modulus squared of the amplitude:

$$
\begin{aligned}
P\left(v_{l} \rightarrow v_{l^{\prime}}\right)=\left|A\left(v_{l}(0,0) \rightarrow v_{l^{\prime}}(L, T)\right)\right|^{2} & =\left|\sum_{k} U_{l k} e^{i \varphi_{k}} U_{l^{\prime} k}^{*}\right|^{2} \\
& =\sum_{k} U_{l k} e^{i \varphi_{k}} U_{l^{\prime} k}^{*} \sum_{j} U_{l j}^{*} e^{-i \varphi_{j}} U_{l^{\prime} j} \\
& =\sum_{j} \sum_{k} U_{l k} U_{l^{\prime} k}^{*} U_{l j}^{*} U_{l^{\prime} j} e^{-i\left(\varphi_{j}-\varphi_{k}\right)} .
\end{aligned}
$$

The phase difference $\varphi_{j}-\varphi_{k}$ between the initial and final states is

$$
\varphi_{j}-\varphi_{k}=\left(E_{j}-E_{k}\right) T-\left(p_{j}-p_{k}\right) L
$$

Assuming relativistic neutrinos, then $T \approx L$, and the momentum is

$$
p_{i}=\sqrt{E_{i}^{2}-m_{i}^{2}}=E_{i} \sqrt{1-\frac{m_{i}^{2}}{E_{i}^{2}}} \approx E_{i}\left(1-\frac{m_{i}^{2}}{2 E_{i}^{2}}\right)
$$

so

$$
\varphi_{j}-\varphi_{k} \approx\left(\frac{m_{k}^{2}}{2 E_{k}^{2}}-\frac{m_{j}^{2}}{2 E_{j}^{2}}\right) L .
$$

To simplify, we assume the energies of the mass states are identical, although we could have equally assumed the momenta of the mass states are the same. We get

$$
\varphi_{j}-\varphi_{k}=\frac{\Delta m_{j k}^{2} L}{2 E}
$$

where $\Delta m_{j k}^{2}=m_{j}^{2}-m_{k}^{2}$ and $E_{k}=E_{j}=E$. 
By expanding out Equation 1.8, recombining terms, and using Equation 1.12,

$$
\begin{aligned}
& P\left(v_{l} \rightarrow v_{l^{\prime}}\right)=\sum_{j}\left|U_{l^{\prime} j}\right|^{2}\left|U_{l j}\right|^{2}+2 \sum_{j>k}\left|U_{l^{\prime} j} U_{l j}^{*} U_{l k} U_{l^{\prime} k}^{*}\right| \times \cos \left(\frac{\Delta m_{j k}^{2} L}{2 E}-\phi_{l^{\prime} l ; j k}\right) \\
& P\left(\bar{v}_{l} \rightarrow \bar{v}_{l^{\prime}}\right)=\sum_{j}\left|U_{l^{\prime} j}\right|^{2}\left|U_{l j}\right|^{2}+2 \sum_{j>k}\left|U_{l^{\prime} j} U_{l j}^{*} U_{l k} U_{l^{\prime} k}^{*}\right| \times \cos \left(\frac{\Delta m_{j k}^{2} L}{2 E}+\phi_{l^{\prime} l ; j k}\right)
\end{aligned}
$$

where $l, l^{\prime}=e, \mu, \tau$ and $\phi_{l^{\prime} l ; j k}=\arg \left(U_{l^{\prime} j} U_{l j}^{*} U_{l k} U_{l^{\prime} k}^{*}\right)$. CPT invariance requires that

$$
P\left(v_{l} \rightarrow v_{l^{\prime}}\right)=P\left(\bar{v}_{l^{\prime}} \rightarrow \bar{v}_{l}\right)
$$

which is assumed to hold. However, the condition for CP invariance

$$
P\left(v_{l} \rightarrow v_{l^{\prime}}\right)=P\left(\bar{v}_{l} \rightarrow \bar{v}_{l^{\prime}}\right)
$$

is only held if $\phi_{l^{\prime} l ; j k}$ is an integer multiple of $\pi$, and hence, if $U$ is real.

In principle, one can calculate the neutrino oscillation probability in vacuum using Equations 1.3, 1.13, and 1.14 and a few parameters:

- the distance from the source to the detector, $L$,

- the neutrino energy, $E$,

- the angles, $\theta_{12}, \theta_{13}$, and $\theta_{23}$,

- the $\mathrm{CP}$ violation phase, $\delta_{C P}$,

- and the neutrino mass squared differences, $\Delta m_{21}^{2}, \Delta m_{31}^{2}$, and $\Delta m_{32}^{2}$, only two of which are independent $\left(\Delta m_{31}^{2}=\Delta m_{21}^{2}+\Delta m_{32}^{2}\right)$.

The oscillation probabilities depend on the mass squared differences, so oscillation experiments on their own cannot elucidate the absolute masses of the different mass states. That is, measuring the vacuum oscillation probabilities in an experiment is not sufficient to determine the absolute neutrino masses, nor which mass states are heavier than the others. Absolute masses can, however, be determined by examining the $\beta$ energy spectrum near the endpoint, for example, in tritium decays [11] or in various astronomical or cosmological observations. The sign of $\Delta m_{21}^{2}$ (also known as $\Delta m_{\text {sol }}^{2}$ ) is known from solar neutrino experiments, but the current data do not restrict the signs of $\Delta m_{31}^{2}$ or $\Delta m_{32}^{2}$ (also known as $\Delta m_{\text {atm }}^{2}$ ). The two options for the mass ordering (or "hierarchy") are commonly referred to as 
the "normal ordering" (NO) where $m_{1}<m_{2}<m_{3}$ and the "inverted ordering" (IO) where $m_{3}<m_{1}<m_{2}$ (see Figure 1.1).

Despite the fact that neutrinos interact with matter very weakly, the oscillation probabilities are affected when passing through dense materials. This is due to coherent elastic scattering of neutrinos on the electrons, protons, and neutrons which make up ordinary matter, where the particle momenta and spin states are unchanged. In particular, $v_{e}, v_{\mu}$, and $v_{\tau}$ all have different scattering amplitudes arising from different indices of refraction [12], and their effective masses are increased by different amounts. Oscillations between certain types of neutrinos can have a resonant amplification of the transition probabilities at specific energies [13]. Additionally, the matter effects on the neutrino oscillations and antineutrino oscillations are different, since there are no natural positrons, antiprotons, or antineutrons on which to scatter. This so-called MSW effect [12, 13] can allow insights into the absolute mass differences between mass states (i.e. whether $m_{3}$ is greater than or less than $m_{1}$ and $m_{2}$ ), despite the vacuum oscillation probabilities depending on the squared mass differences.

As an example, the $v_{\mu} \rightarrow v_{e}$ oscillation probability is

$$
\begin{aligned}
P\left(v_{\mu} \rightarrow v_{e}\right) \simeq & \sin ^{2} \theta_{23} \frac{\sin ^{2} 2 \theta_{13}}{(A-1)^{2}} \sin ^{2}[(A-1) \Delta] \\
& +\alpha^{2} \cos ^{2} \theta_{23} \frac{\sin ^{2} 2 \theta_{12}}{A^{2}} \sin ^{2}(A \Delta) \\
& -\alpha \frac{8 J_{C P}}{A(1-A)}(\sin \Delta)(\sin A \Delta)(\sin [(1-A) \Delta]) \\
& +\alpha \frac{8 J_{C P} \cot \delta_{C P}}{A(1-A)}(\cos \Delta)(\sin A \Delta)(\sin [(1-A) \Delta])
\end{aligned}
$$

where $\alpha=\Delta m_{21}^{2} / \Delta m_{31}^{2}, \Delta=\Delta m_{31}^{2} L / 4 E, A=\sqrt{2} G_{F} N_{e} 2 E / \Delta m_{31}^{2}, G_{F}$ is the Fermi constant, $N_{e}$ is the number density of electrons in the medium, and $J_{C P}=\operatorname{Im}\left(U_{\mu 3} U_{e 3}^{*} U_{e 2} U_{\mu 2}^{*}\right)$ [8]. The probability $P\left(\bar{v}_{\mu} \rightarrow \bar{v}_{e}\right)$ is equivalent to Equation 1.17 apart from switching the signs of $A$ and $J_{C P}$. That is, the matter effect and CP violation are both causes for possible asymmetries in the neutrino and antineutrino oscillation channels, which would be evidence for CP violation in the lepton sector.

Table 1.1 shows the current global best fit values of the three flavour oscillation parameters.

\subsubsection{Remaining Unanswered Questions}

There are still many remaining unanswered questions and measurements to be made regarding neutrino oscillations. First, are neutrinos Dirac fermions or Majorana particles? If the former, a neutrino $v_{l}$ has a distinctive antiparticle $\bar{v}_{l}$ with opposite lepton charge. On the other hand, 

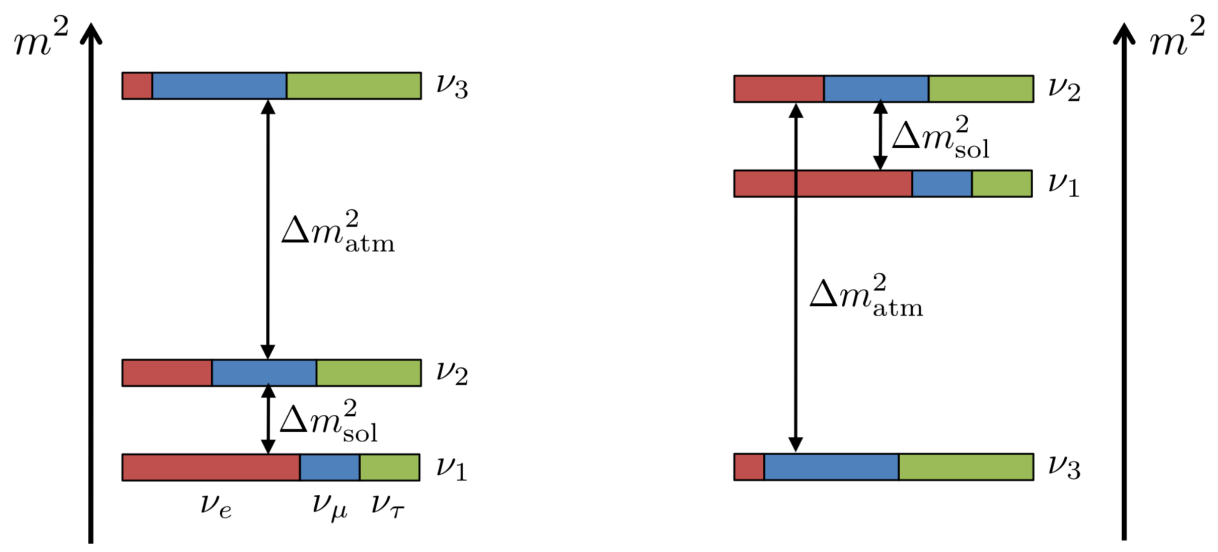

Fig. 1.1 Two possible orderings of the neutrino mass squared differences. Left: NO, right: IO.

Table 1.1 Current neutrino oscillation global best fit parameter values and $3 \sigma$ ranges (from [14]). For $\delta / \pi$, the $2 \sigma$ range is shown as no values for $\delta$ are disfavoured at the $3 \sigma$ level. Values in the table are given for NO neutrino masses $\left(m_{1}<m_{2}<m_{3}\right)$. Values in brackets are given for IO neutrino masses $\left(m_{3}<m_{1}<m_{2}\right)$. Also, $\Delta m^{2}=m_{3}^{2}-\left(m_{2}^{2}+m_{1}^{2}\right)$ which is positive for $\mathrm{NO}$ and negative for IO.

\begin{tabular}{lll}
\hline Parameter & Best fit & $3 \sigma$ \\
\hline$\Delta m_{21}^{2}\left[10^{-5} \mathrm{eV}^{2}\right]$ & 7.37 & $6.93-7.97$ \\
$\left|\Delta m^{2}\right|\left[10^{-3} \mathrm{eV}^{2}\right]$ & $2.50(2.46)$ & $2.37-2.63(2.33-2.60)$ \\
$\sin ^{2} \theta_{12}$ & 0.297 & $0.250-0.354$ \\
$\sin ^{2} \theta_{23}, \Delta m^{2}>0$ & 0.437 & $0.379-0.616$ \\
$\sin ^{2} \theta_{23}, \Delta m^{2}<0$ & 0.569 & $0.383-0.637$ \\
$\sin ^{2} \theta_{13}, \Delta m^{2}>0$ & 0.0214 & $0.0185-0.0246$ \\
$\sin ^{2} \theta_{13}, \Delta m^{2}<0$ & 0.0218 & $0.0186-0.0248$ \\
$\delta / \pi$ & $1.35(1.32)$ & $0.92-1.99(0.83-1.99)$
\end{tabular}


if lepton charge is not conserved and the $v_{l}$ and $\bar{v}_{l}$ can be interchanged in any interaction, the neutrino is said to be Majorana. If Majorana, the oscillation matrix, $U$ (Equation 1.3), will have two extra CP violation phases, $\alpha_{21}$ and $\alpha_{31}$,

$$
U_{\text {Majorana }}=U \times \operatorname{diag}\left(1, e^{i \alpha_{21} / 2}, e^{i \alpha_{31} / 2}\right) .
$$

The current experimental data is not sufficient to distinguish the Dirac or Majorana nature of neutrinos, and oscillation experiments are not sensitive to the Majorana phase. Only $0 v \beta \beta$ decay experiments could possibly be sensitive under the current limits [15]. The absolute neutrino masses are also out of reach for oscillation experiments, but experiments to determine the rate of $0 v \beta \beta$ decays, experiments analysing the ${ }^{3} \mathrm{H} \beta$ decay endpoint, as well as cosmological or astronomical observations may shed light on these fundamental values.

Also under question is the status of CP symmetry in leptons. CP symmetry holds if the $\delta_{C P}$ phase parameter is an integer multiple of $\pi$, and broken otherwise. The value of $\delta_{C P}$ is directly testable through analysing the symmetry of oscillation probabilities between specific flavours of neutrinos and the same flavoured oscillations between antineutrinos, such as Equation 1.16 for $l \neq l^{\prime}$.

As shown in Table 1.1, the absolute values of the mass squared differences $\Delta m_{21}^{2}$ and $\Delta m_{31}^{2}$ are known experimentally, but the sign of $\Delta m_{31}^{2}$ is not. The sign of $\Delta m_{31}^{2}$ will be measured in parallel with $\delta_{C P}$, as they both contribute to the symmetry (or asymmetry) of the oscillation probability in Equation 1.16.

While the SM contains three neutrino flavours, the number of flavours can, in general, be larger than three. The existence of one or two sterile neutrinos, indicated primarily by an excess of $\bar{v}_{\mu} \rightarrow \bar{v}_{e}$ events, is suggested with statistical significance by the experiments LSND [16] and MiniBooNE [17], though it has been suggested that an incorrectly estimated electron-like background might account for the discrepancy [18]. If they exist, these neutrinos would have opposite helicity to, and would mix with, the known flavour neutrinos. Several dedicated experiments are planned to test this hypothesis with greater sensitivity, for example, the Short Baseline Neutrino (SBN) programme at Fermilab [19].

\subsection{Energy Loss In Matter}

Energetic particles are detected by transferring some or all of their energy to the detection medium. The physical mechanisms of particle energy loss vary depending on the type of incident particle, the amount of energy transferred, and the properties of the medium. Massive charged particles can lose energy through collisions with the atoms in the medium, creating 
detectable event signatures. Also, accelerating particles or deexciting atoms may radiate energy as photons. At high energies, particles may also interact with nuclei and produce a different type of detection signature. The theory which describes the various forms of energy loss in matter is essential in understanding and interpreting the signals created in particle detectors.

\subsubsection{Charged Particle Energy Loss}

Energetic charged particles travelling in a dense medium interact via the Coulomb force with nuclei or orbital electrons. In each collision, the primary particle loses energy through ionisation and excitation. The Bethe equation [20] describes the mean incident particle energy $E$ loss per unit length $x$ (or "mass stopping power"):

$$
-\frac{d E}{d x}=\frac{4 \pi e^{4} z^{2} N Z}{m_{e} v^{2}}\left[\ln \frac{2 m_{e} v^{2}}{I}-\ln \left(1-\beta^{2}\right)-\beta^{2}\right],
$$

where $v=\beta c$ and $z e$ are the incident particle velocity and charge, $N$ and $Z$ are the absorber number density and atomic number, $m_{e}$ is the electron rest mass, $e$ is the elementary charge, and $I$ is the mean excitation energy which depends on the atomic properties of the elements in the medium. This formula remains generally valid for various different incident particles and various target materials, and can be used to derive expressions for particle range and stopping time in the absorber, and total energy deposit (dose).

Energetic electrons (or any of the charged leptons, in general) radiate energy in the form of photons in addition to collisional energy losses. This radiation, known as bremsstrahlung, is released as a result of the incident particle accelerating when deflected in the Coulomb field of the nucleus or the atomic shell electrons of the medium. Similar to Equation 1.19, Bethe parameterised the total energy loss for electrons as a sum of the specific energy loss due to collisions and the specific energy loss due to radiation [20]

$$
\frac{d E}{d x}=\left(\frac{d E}{d x}\right)_{c}+\left(\frac{d E}{d x}\right)_{r}
$$

where the specific collisional (ionisation and excitation) energy loss for electrons is

$$
\begin{array}{r}
-\left(\frac{d E}{d x}\right)_{c}=\frac{2 \pi e^{4} N Z}{m_{e} v^{2}}\left(\ln \frac{m_{e} v^{2} E}{2 I^{2}\left(1-\beta^{2}\right)}-(\ln 2)\left(2 \sqrt{1-\beta^{2}}-1+\beta^{2}\right)\right. \\
\left.+\left(1-\beta^{2}\right)+\frac{1}{8}\left(1-\sqrt{1-\beta^{2}}\right)^{2}\right)
\end{array}
$$


and the contribution to the total energy loss by bremsstrahlung radiation is

$$
-\left(\frac{d E}{d x}\right)_{r}=\frac{N E Z(Z+1) e^{4}}{137 m_{e}^{2} c^{4}}\left(4 \ln \frac{2 E}{m_{e} c^{2}}-\frac{4}{3}\right) .
$$

The ratio of energy losses to the different processes is approximately

$$
\frac{(d E / d x)_{r}}{(d E / d x)_{c}} \approx \frac{E Z}{700}
$$

showing that radiative energy loss by electrons is a small fraction of the total energy loss except for high energy electrons and high $Z$ absorber materials. For example, electron energy loss in copper is shown in Figure 1.2 showing collisional energy loss dominating at low energies, and bremsstrahlung radiation dominating at high energies.

One main drawback of the Bethe theory of energy loss is that Equation 1.19 describes the mean energy loss of a particle in a medium over a given distance. However, in a given interaction of a particle with a medium, the energy loss follows an asymmetric LandauVavilov-Bichsel distribution [12], which is not well described by the mean value due to the very long upper tail. Instead, the most probable energy loss, $\Delta_{p}$, is a better metric for describing fractional energy loss of a minimally ionising particle,

$$
\frac{\Delta_{p}}{x}=\xi\left[\ln \frac{2 m_{e} c^{2} \beta^{2}}{I\left(1-\beta^{2}\right)}+\ln \frac{\xi}{I}+j-\beta^{2}-\delta(\beta \gamma)\right],
$$

where $\xi=2 \pi N_{A} r_{e}^{2} m_{e} c^{2}\langle Z / A\rangle\left(z^{2} / \beta^{2}\right), j=0.200$ [12], $\delta(\beta \gamma)$ is a function which corrects for the density effect of the medium, $N_{A}$ is the Avogadro number, $r_{e}$ is the classical electron radius, and $A$ is the atomic mass of the medium. The difference between the mean and most probable energy loss is shown in Figure 1.3.

\subsubsection{Photon Interactions in Matter}

Photons interact with matter through several different mechanisms which depend on the photon energy and absorber material properties. Figure 1.4 shows the cross-sections for various photon interaction mechanisms in carbon and lead. In regards to radiation detection and measurement, three mechanisms dominate: photoelectric absorption, Compton scattering, and pair production.

For low energy photons, the dominant interaction mechanism is photoelectric absorption, which is when a photon is absorbed by an atom, causing it to excite and release an electron 


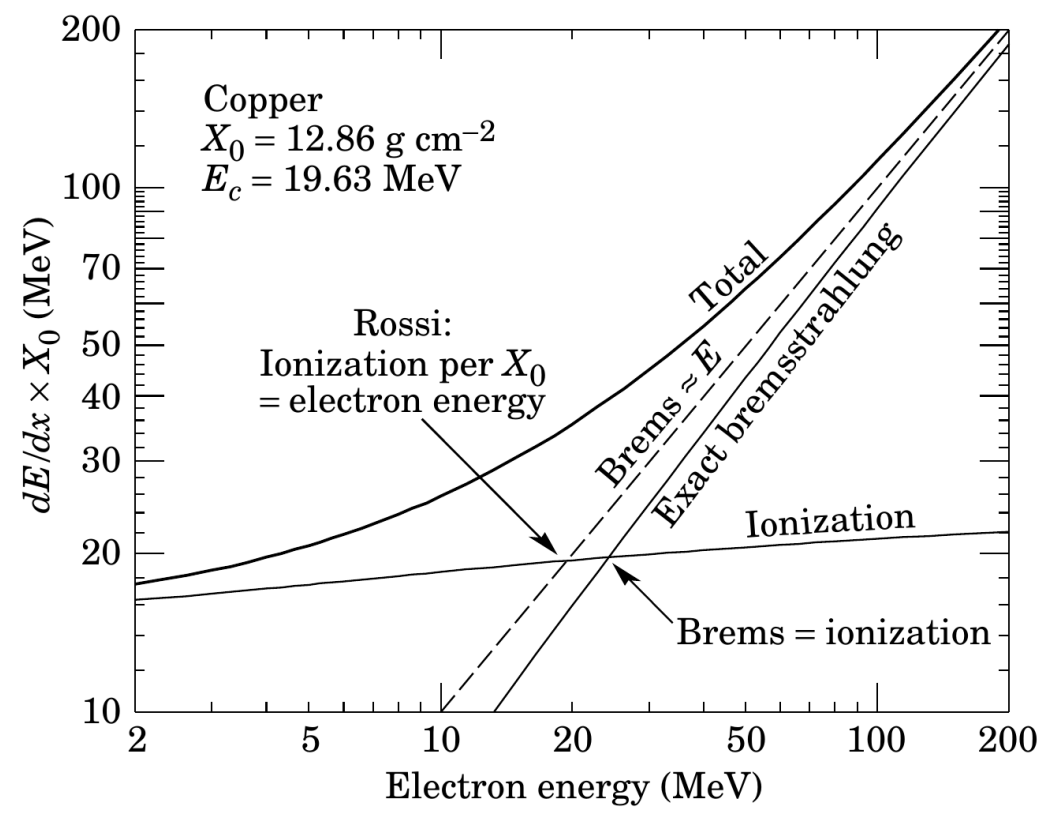

Fig. 1.2 Energy loss in copper as a function of electron energy [8]. For low energies, ionisation processes dominate, whereas for larger energies, radiative effects dominate.

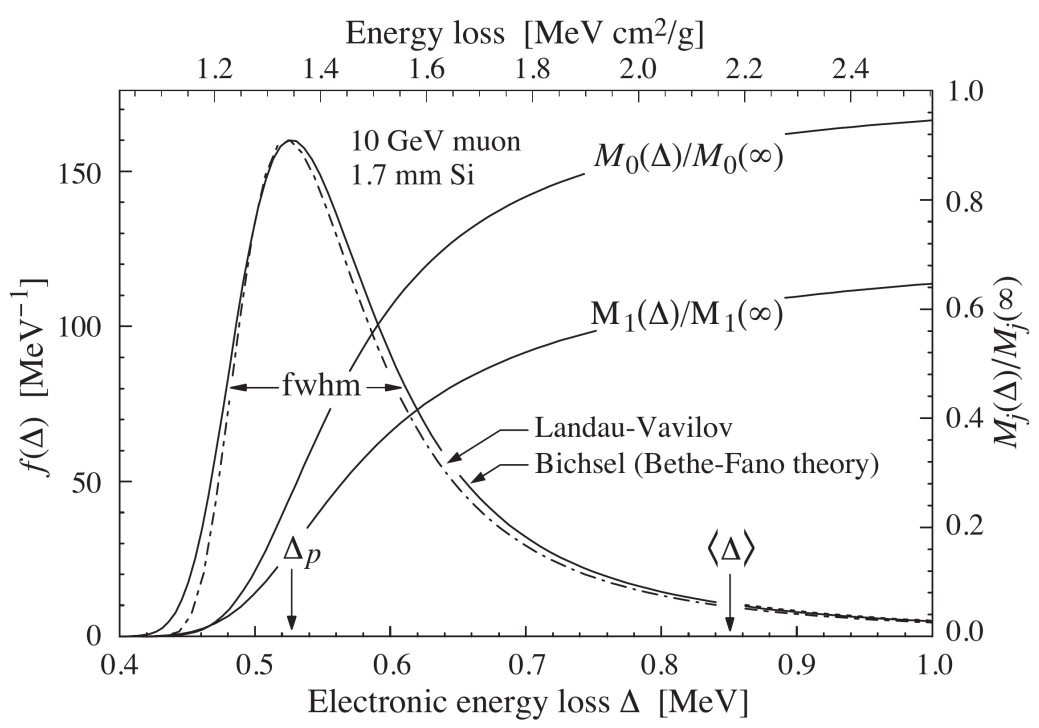

Fig. 1.3 Probability distribution of energy loss $\Delta$ from two theories (Landau-Vavilov and Bethe-Fano). Mean energy loss, $\langle\Delta\rangle$, and most probable energy loss, $\Delta_{p}$, are indicated. $M_{0}(\Delta)$ and $M_{1}(\Delta)$ are the cumulative 0th moment (mean number of collisions) and cumulative 1st moment (mean energy loss) in crossing the detector [8]. 


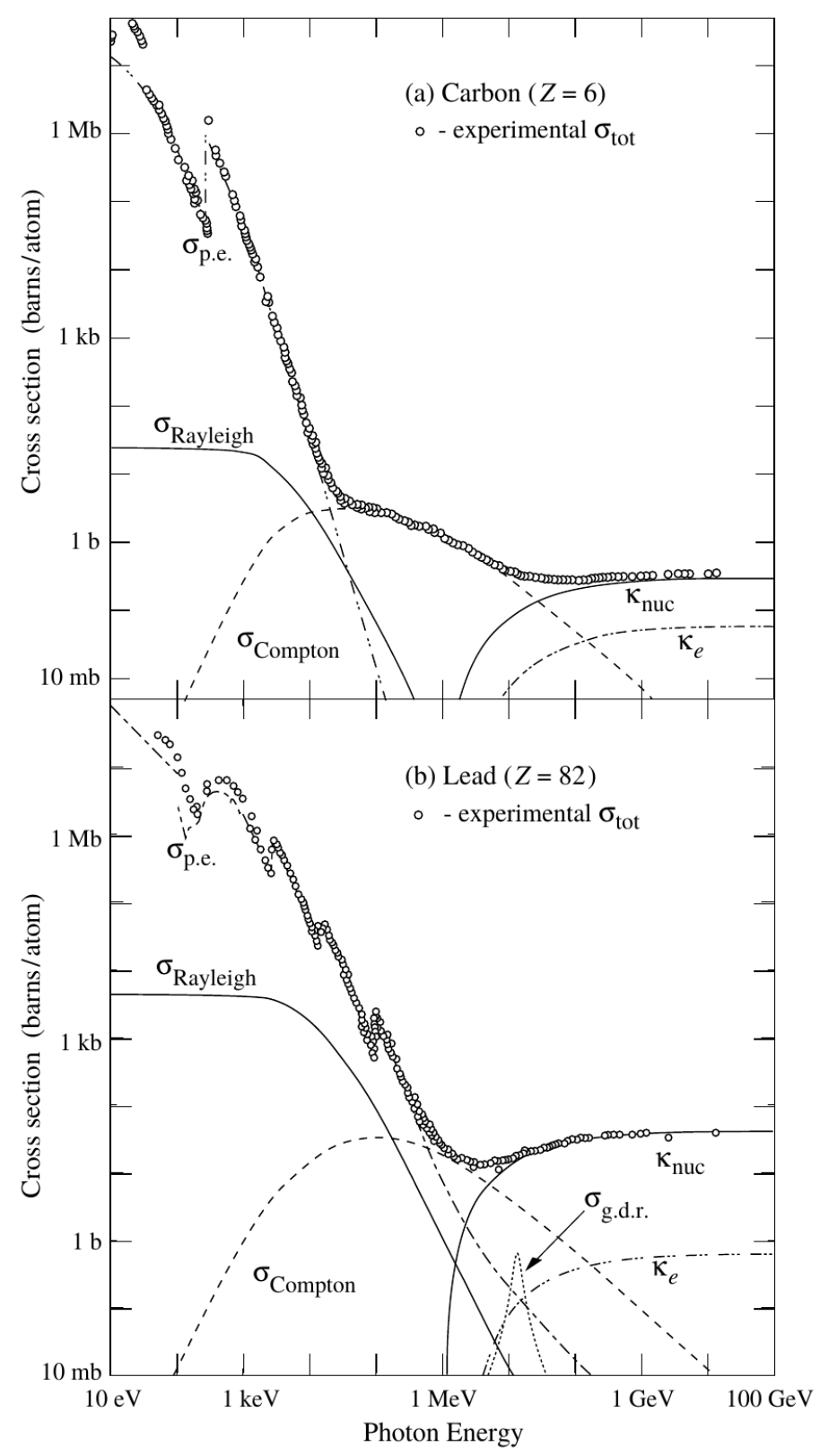

Fig. 1.4 Photon total cross-sections as a function of energy in carbon and lead [8]. Different processes are shown: $\sigma_{\text {p.e. }} \rightarrow$ photoelectric effect, $\sigma_{\text {Rayleigh }} \rightarrow$ Rayleigh (coherent) scattering, $\sigma_{\text {Compton }} \rightarrow$ Compton (incoherent) scattering, $\kappa_{\text {nuc }} \rightarrow$ nuclear field pair production, $\kappa_{e} \rightarrow$ electron field pair production, and $\sigma_{\text {g.d.r. }} \rightarrow$ photonuclear interactions. 
from one of its bound shells. The kinetic energy of the released electron is

$$
E=h v-\phi_{w}
$$

where $\phi_{w}$ is called the work function which represents the binding energy of the photoelectron in its original shell. The hole left by the ejected electron is quickly filled with another electron, releasing either an X-ray or an Auger electron. The resulting X-ray may interact again releasing more loosely bound electrons, possibly causing several electrons to be extracted from the atom as a result of one absorbed photon. This secondary release of electrons can be seen in Figure 1.4 where the cross-section shows sudden jumps. These jumps are attributed to the $K, L, M, \ldots$ shell electrons being ejected, the discontinuous behaviour relating to the specific binding energy of the shells.

At slightly higher energies, Compton scattering dominates the photon interactions. This occurs when an energetic photon is deflected through an angle $\theta$ by an outer shell electron, which recoils with some kinetic energy. The transfer of energy can range from zero to a large fraction of the initial photon energy. By considering the conservation of energy and momentum in the two body scattering problem, the relationship between the initial $(h v)$ and final $\left(h v^{\prime}\right)$ photon energy and the scattering angle $\theta$ is given by

$$
h v^{\prime}=\frac{h v}{1+\frac{h v}{m_{e}}(1-\cos \theta)}
$$

with the maximum energy transfer at $\theta=\pi$. For very small scattering angles, the energy transfer is minimal and goes abruptly to zero. Since it is impossible for the photon to transfer more than $h v$ energy, there is a sharp cutoff in the energy distribution, called the Compton edge.

If the photon energy exceeds $2 m_{e}$, production of an electron-positron pair is physically possible. Within the Coulomb field of a nucleus, the photon may disappear, creating a $e^{+} e^{-}$pair, each carrying as kinetic energy half of the excess energy above the required $2 m_{e}$ threshold (neglecting a tiny amount of recoil energy carried away by the nearby nucleus). The resulting charged particles will lose energy through ionisation and excitation before annihilating, releasing two back-to-back $511 \mathrm{keV}$ photons.

\subsubsection{Other Forms of Particle Interactions with Matter}

Other important mechanisms of particle interactions in dense materials are Rayleigh scattering, Cherenkov radiation, electronic and hadronic showers, and nuclear interactions. 
Photons may scatter coherently with the electrons in an absorber atom. This so-called Rayleigh scattering is usually not a significant mechanism for photon energy loss because almost no energy is transferred from the photon to the electrons. All of the transferred energy goes to causing the atom as a whole to recoil, and none goes to ionising or exciting the atom or any around it. This process is only significant in the very low photon energy regime, and even then is not very relevant regarding creating a useful signal in a detector.

Cherenkov radiation is important to neutrino physics as several major oscillation experiments use this mechanism as the primary detection channel. It occurs when a charged particle is travelling faster than the local phase velocity of light. In these interactions, photons are emitted at angle $\theta_{c}$ from the incident charged particle track, azimuthally isotropic around the trajectory. Due to the particle velocity $\beta$, this creates a cone of light with a particular opening half-angle, $\theta_{c}$ which is related to the index of refraction of the material, $n$, [21]

$$
\cos \theta_{c}=\left(\frac{1}{n \beta}\right)
$$

Other properties of the detected light cone are important for performing particle identification, for example, as electrons and muons project fuzzy and sharp, respectively, rings of light on the detector wall.

If a particle or photon has high enough energy when entering a dense absorber, it may initiate an electromagnetic cascade whereby pair production and bremsstrahlung processes create more and more electrons and photons with lower energy. The "shower" is vital in particle accelerator detectors as the total energy of the incident particle can be deduced by the shape of the resulting ionisation cone left behind in the absorber.

In the extremely high energy regime $\left(>10^{20} \mathrm{eV}\right)$, photons and electrons are more likely to interact with the protons and neutrons in a nucleus. These interactions are hadronic and can have many possible outcomes.

\subsection{Neutrino Interactions with Matter}

Neutrinos interact with normal matter via the exchange of a $W^{ \pm}$or $Z$ boson, which are called "charged current interactions" and "neutral current interactions", respectively. The simplest such interactions are scattering reactions, such as the neutrino-electron scattering

$$
\left(\vec{v}_{l}\right)+e^{-} \rightarrow \stackrel{(-)}{v_{l}}+e^{-}
$$


for any flavour of neutrino $l=e, \mu, \tau$ or antineutrino. This process has no energy threshold as the final state is the same as the initial state and the only effect is the transfer of kinetic energy from the neutrino to the electron. Therefore, these interactions are important in total neutrino flux experiments, such as solar and supernova neutrino detection. The interaction signature is an energetic electron which may be detected through ionisation, excitation, or radiation, as above. Neutrinos may also scatter elastically with nucleons:

$$
\stackrel{(-)}{v_{l}}+N \rightarrow \stackrel{(-)}{v_{l}}+N
$$

where $N=n, p$.

There is another class of processes where the neutrino interacts with either a charged lepton, nucleon, or a nucleus where the final state components are different from the initial state components. For example, muon neutrinos may interact with electrons via the quasielastic charged current process

$$
v_{\mu}+e^{-} \rightarrow v_{e}+\mu^{-}
$$

which is essentially the inverse of muon decay. This process is considered "quasielastic" because the initial and final states contain one charged and one uncharged lepton, but with changing flavours. Due to the amount of energy required to produce a muon, the neutrino energy threshold for this process is $10.92 \mathrm{GeV}$ [22]. In general, the initial neutrino energy threshold $E_{v}^{\text {th }}$ for an interaction with a target particle $A$ to release $i$ products of type $X_{i}$,

$$
v+A \rightarrow v^{\prime}+\sum_{i} X_{i}
$$

is given by

$$
E_{v}^{\text {th }}=\frac{\left(\sum_{i} m_{i}\right)^{2}}{2 m_{A}}-\frac{m_{A}}{2},
$$

where $m_{A}$ is the mass of particle $A$, and $m_{i}$ is the mass of particle $X_{i}$. Neutrinos and antineutrinos of all flavours $l=e, \mu, \tau$ may also interact with nucleons

$$
\begin{gathered}
v_{l}+n \rightarrow p+l^{-} \\
\bar{v}_{l}+p \rightarrow n+l^{+},
\end{gathered}
$$

but with different energy thresholds (see [22] for examples). Reaction 1.33 is a $\beta$ decay where the neutrino has been switched from the final state to the initial state, and 1.34 is known as inverse $\beta$ decay for $l=e$ and was used in the Reines and Cowan discovery of the neutrino [2]. Generally speaking, the quasielastic $\mathrm{CC}$ reactions on detector nuclei have small threshold neutrino energies so are commonly used in experiments. For example, the reaction 
$v_{e}+{ }^{40} \mathrm{Ar} \rightarrow e^{-}+{ }^{40} \mathrm{~K}$ has threshold energy of $2.02 \mathrm{MeV}$. These interactions typically leave the resulting nucleus or nucleon in an excited state, which decays producing further detection opportunities.

The final neutrino-matter interaction category is called deep inelastic scattering (DIS) and is denoted by the CC interactions

$$
\begin{gathered}
v_{l}+N \rightarrow l^{-}+X \\
\bar{v}_{l}+N \rightarrow l^{+}+X
\end{gathered}
$$

where $X$ denotes any set of final hadrons. Similarly, NC DIS is also possible:

$$
\stackrel{(-)}{v_{l}}+N \rightarrow \stackrel{(-)}{v_{l}}+X
$$

These interactions require very high neutrino energy and, thus, can produce very complicated final states which are difficult to reconstruct.

\subsection{Summary}

The current state of knowledge regarding neutrino oscillations and neutrino detection has advanced dramatically since 1930 when the neutrino was first postulated. While the above theory has attempted to provide a fundamental general background, more information is widely available which goes into much more depth regarding neutrino oscillations, particle interactions with matter, and neutrino detection (for example, $[8,22]$ ). Ultimately, the goal of this thesis is to overcome some of the challenges in building massive LAr TPCs for detecting neutrinos of all flavours and with a wide range of energies, and the theory presented here provides a strong motivation for performing the detector development. 



\section{Chapter 2}

\section{Liquid Argon Time Projection Chambers}

In 1975, Nygren and others [23, 24] proposed the time projection chamber (TPC), combining the multiwire proportional chamber [25] with the drift chamber [26, 27]. The TPC allows simultaneous readout of three-dimensional coordinates of each point on a particle track, which is especially important in high multiplicity events and is a significant improvement over previous $2 \mathrm{D}$ readout methods such as bubble and spark chambers. Furthermore, by choosing an appropriate interaction medium, accurate measurements of particle energy loss at every point along a track allow particle identification.

For the last 60 years or so, noble gases, liquids, and solids have been used as high energy particle detectors. Alvarez first suggested [28] that the scintillation and ionisation properties of liquid and solid argon [29,30] could be utilised in a continuous use high-resolution particle tracking detector. Rubbia was the first to suggest using liquid argon (LAr) as the interaction medium in a TPC for neutrino detection [31], which has been intensely studied since then. The high density of LAr and its excellent charge transport properties motivate the use of LAr as a TPC detection medium for neutrino and dark matter detection experiments.

This chapter will describe the components of a LAr TPC and operating principles. Also, the benefits and drawbacks of using LAr as a detection medium are discussed in the context of operating a successful TPC experiment. Finally, the history and current status of LAr TPCs in neutrino physics is presented, with a particular focus on the Deep Underground Neutrino Experiment (DUNE).

\subsection{LAr TPC Description and Operating Principle}

As illustrated in Figure 2.1, energetic charged particles interacting in LAr ionise the argon atoms, leaving behind an "image" of free electrons along the particle track. This image is drifted in a uniform electric field toward the readout anode in the TPC, which can be 
reconstructed as a three-dimensional image of the event using a two-dimensional readout plus drift time. Some TPCs include light detection for measuring the prompt scintillation light from the primary interaction, which gives a reference for converting drift time to a position along the drift. Details of the physics of these processes and some important considerations are discussed in Section 2.2.

The basic components of a LAr TPC include:

- a cathode charged with negative high voltage,

- an anode with grounded charge coupled readout, typically in the form of wires,

- a volume of LAr between cathode and anode in which interactions take place and ionisation charge is drifted,

- and a 3D readout of ionisation signals at the anode (2 spatial coordinates + drift time).

Field shaping rings are used to achieve a uniform electric field in the active volume. The rings are held at monotonically decreasing negative voltages to create a constant potential gradient from the cathode to the anode.

Most TPCs use a wire-based scheme for reading out charge signals [33], although there is significant current interest in using pixel-based readout in a LAr TPC (for example, [34]). A typical TPC readout consists of a Frisch grid [35] to shield the sense wires from induction from far away moving charges and several layers of sense wires oriented at different angles for $2 \mathrm{D}$ coordinate determination. The DUNE standard design uses four layers. A Frisch grid wire plane shields the sense wires from positive ion drift induction. Two "induction" planes, where the wires run at different angles to each other (e.g. $\pm 45^{\circ}$ ), measure bipolar induction signals by charge passing through the planes. Finally, one anode "collection" plane of wires (e.g. at $0^{\circ}$ ) measure unipolar signals by charge coming to rest at the end of the electric field lines [36]. By measuring charge induction signals from each of the three sense wires, an accurate 2D measurement of the location of ionisation can be made since there is usually only one possible point of intersection between the wires in all three planes.

By applying appropriate voltages to the consecutive wire planes, the electric fields can be designed such that no field lines terminate on the induction wires, i.e. electrons may pass through induction planes with $100 \%$ transparency. The requirement on the fields in two adjacent regions is given by [37]

$$
\frac{1-\rho}{1+\rho}<\frac{E_{2}}{E_{1}}<\frac{1+\rho}{1-\rho}
$$

where $\frac{E_{2}}{E_{1}}$ is the ratio of fields on either side of the induction wire plane, $\rho=\frac{2 \pi r}{a}, r$ is the wire radius, and $a$ is the wire separation distance in the plane. 


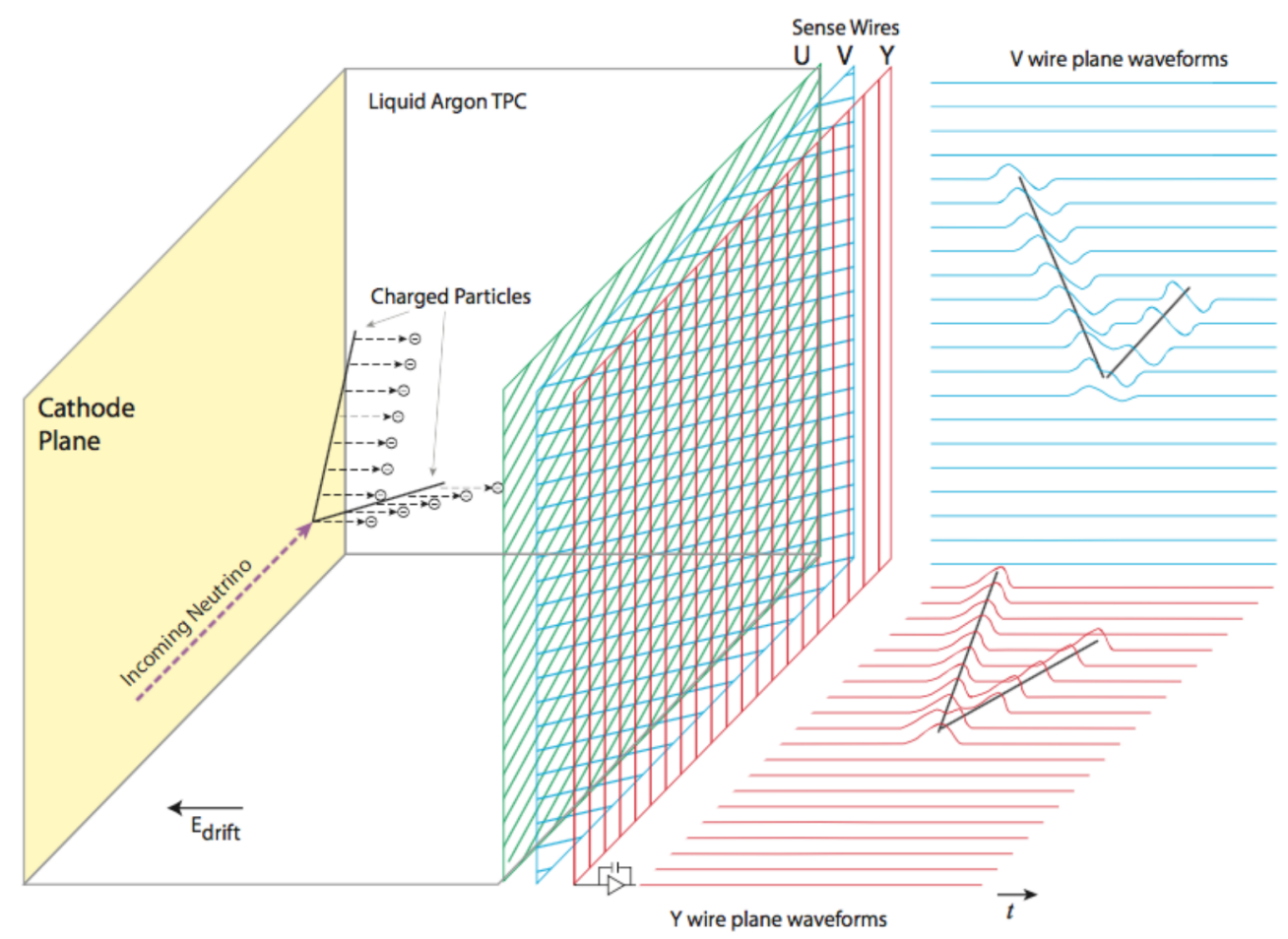

Fig. 2.1 Schematic of the elements and operation of a LAr TPC [32].

In addition to charge readout via wires, scintillation light is valuable in operating a LAr TPC. Photodetectors which are sensitive to vacuum ultraviolet (VUV) light provide event timing information, as well as rough position information as a result of varying attenuation of light to photodetectors at different distances from the original ionisation event location. While the scintillation light output in an event is proportional to the particle energy loss in the LAr, the light output is not typically used for calorimetric purposes as there is rarely enough sensitive detection area to attain a reasonable energy resolution for measurement [33].

\subsubsection{Dual Phase LAr TPC}

Under the influence of an electric field, primary ionisation electrons drifting in a noble gas gain energy and, if the kinetic energy is high enough, can cause further ionisation and excitation, releasing more electrons. The secondary electrons also gain energy and can further ionise and excite the argon, leading to an avalanche process. This multiplication process is suppressed in LAr as the maximum achieved gain was shown to be 150 [38], whereas gains greater than 700 are possible in the gas phase [39]. The idea then emerged [40] to combine the LAr TPC with the electron multiplication in GAr. That is, use LAr as a particle detection medium because of its high density and charge production and transport qualities, extract the 
electrons from the liquid into the gas phase, and amplify the signal using a strong electric field. This process results in an increased signal-to-noise ratio of the readout allowing smaller observable signals and longer drift distances in LAr. The idea has been successfully pursued by several neutrino and dark matter detection experiments (see [34, 41, 42]).

In a dual phase LAr TPC, it is typical for the readout of charge signals to be a strip-based projective readout (for example, [43]). In addition to the charge, primary and secondary scintillation light are collected by photomultiplier tubes (PMT), which is used to determine the coordinate of the ionisation along the drift direction (for example, [44]), as well as for other purposes such as $e^{-} / \gamma$ discrimination.

\subsubsection{Signal Generation}

Signals in a TPC are created by induction from moving charges near the readout electrodes. The Shockley-Ramo theorem $[45,46]$ states that the current induced, $i$, is given by

$$
i=Q \vec{v} \cdot \vec{E}_{0}(\vec{x})
$$

where $Q$ is the charge (assumed to be point-like), $\vec{v}$ is the velocity of the charge, and $\vec{E}_{0}(\vec{x})$ is a weighting field calculated assuming $1 \mathrm{~V}$ potential on the electrode and $0 \mathrm{~V}$ on all other electrodes. Typically, $\vec{E}_{0}(\vec{x})$ is non-trivial to calculate, and is usually determined through electrodynamic simulations. For example, Figure 2.3 shows a simulated weighting field for a LAr TPC under development.

\subsection{Physics of LAr Detectors}

LAr is well suited for use as a high energy particle detection medium for many reasons, including its high density, its very high electron mobility [48], its inertness resulting from a filled outer shell of electrons, and its natural abundance (1\% of air). Some properties of LAr are shown in Table 2.1. Some drawbacks include the strong dependence of the electron drift lifetime on the electronegative impurity concentration and the engineering requirements to purify and maintain large quantities for experimentation, but these problems are mostly solved $[49,50]$.

Generally, LAr is important because of its ability to absorb ionising radiation due to its short radiation length, indicating that charged particles are easily stopped by LAr. LAr also has a significant ionisation electron yield from moderately energetic particle interactions given its low energy required to create an ion pair and high stopping power. Also, LAr is mostly transparent to its VUV scintillation light $(127 \mathrm{~nm})$ due to its long attenuation length. 


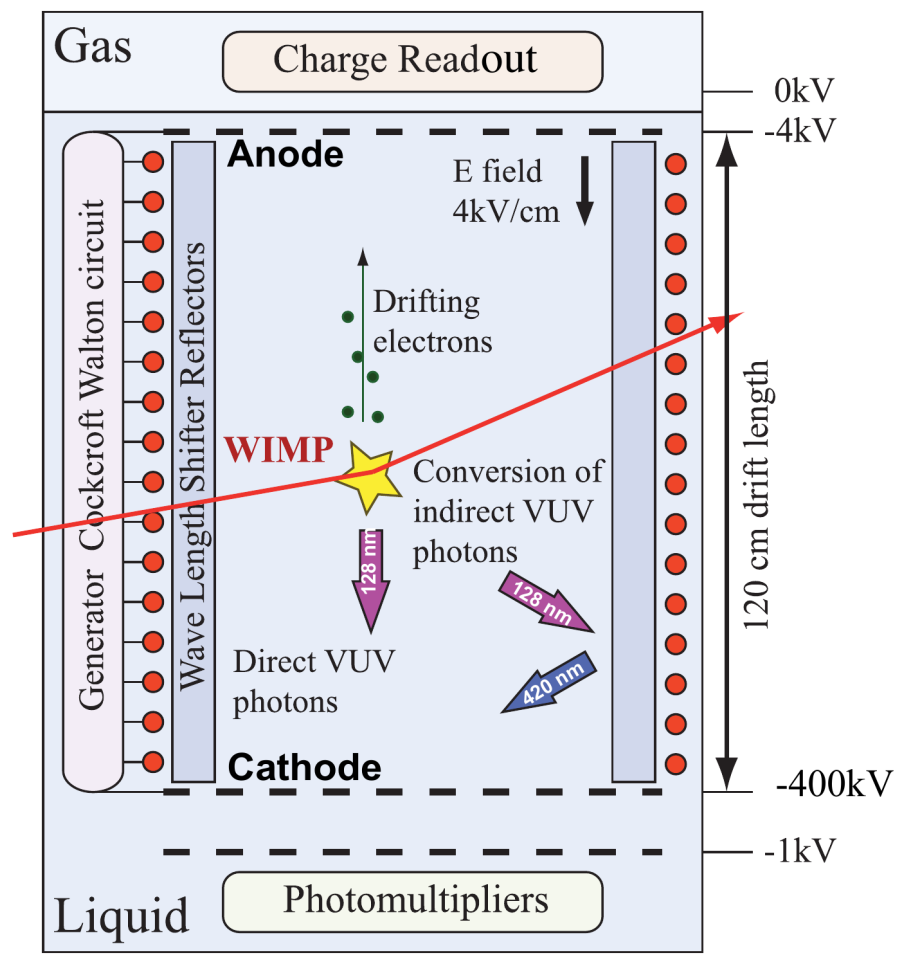

Fig. 2.2 Illustration of the ArDM two-phase argon TPC [44]. Red line indicates the trajectory of a primary particle interacting, leaving a trail of ionisation and a burst of light by the resulting energetic charged particle. The charge is drifted upwards by an electric field and extracted across the liquid-gas boundary to be read out. Amplification occurs in the gas phase, where additional argon scintillation light is produced. PMTs collect the light.

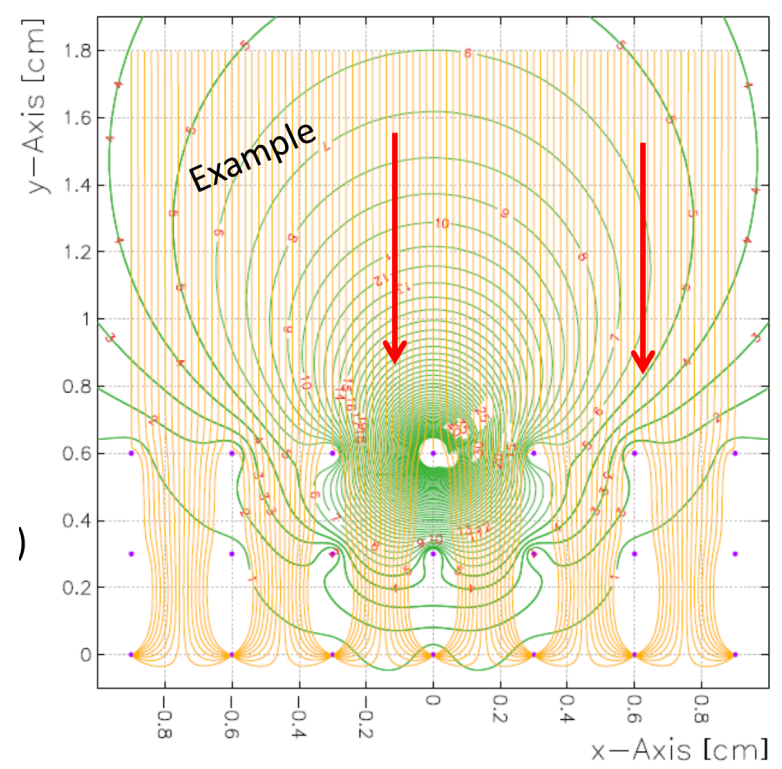

Fig. 2.3 Weighting field on a TPC induction wire (contours), and electric field lines when fully biased (yellow lines) [47]. 
Table 2.1 Physical properties of LAr $[51,52,53,54,55,56,57,58,59,60,61,62,63,64$, $65,66]$. $\dagger$ : Assuming density of $1.403 \mathrm{~g} \mathrm{~cm}^{-3} . \diamond$ : Assuming $50 \mathrm{ppb}$ impurity concentration, mainly $\mathrm{O}_{2}, \mathrm{H}_{2} \mathrm{O}$.

Atomic Number

Atomic Mass

Boiling Point

Melting Point

Index of Refraction (128 nm)

Density

Average energy per ion pair

Average energy per photon

Peak VUV scintillation wavelength

FWHM VUV scintillation wavelength

Singlet Decay Time

Triplet Decay Time

VUV Scintillation Attenuation Length ${ }^{\diamond}$

Stopping Power $\langle d E / d x\rangle(\mathrm{MIP})^{\dagger}$

Ionisation Energy

Radiation Length $^{\dagger}$

Moliere Radius ${ }^{\dagger}$

Nuclear Interaction Length ${ }^{\dagger}$

Mass Attenuation Coefficient for $1 \mathrm{MeV} \gamma$

Electron Critical Energy

Electron Mobility (Zero E-field)

Ion Mobility
18

39.948

87.3

83.79

1.38

$1.403 \quad \mathrm{~g} \mathrm{~cm}^{-3}$

$23.6 \pm 0.3 \quad \mathrm{eV}$

$25 \mathrm{eV}$

$127 \quad \mathrm{~nm}$

$\sim 10 \quad \mathrm{~nm}$

$7 \quad$ ns

1600 ns

$52.1 \mathrm{~cm}$

$2.105 \quad \mathrm{MeV} \mathrm{cm}^{-1}$

13.6-13.8 eV

$14 \mathrm{~cm}$

$9.043 \mathrm{~cm}$

$85.77 \quad \mathrm{~cm}$

$0.05762 \quad \mathrm{~cm}^{2} \mathrm{~g}^{-1}$

$32.84 \quad \mathrm{MeV}$

$518 \quad \mathrm{~cm}^{2} \mathrm{~V}^{-1} \mathrm{~s}^{-1}$

$6.61 \times 10^{-4} \mathrm{~cm}^{2} \mathrm{~V}^{-1} \mathrm{~s}^{-1}$ 


\subsubsection{Ionisation and Scintillation}

Charged particles which traverse a LAr cell transfer energy primarily through ionisation and excitation. The amount of energy lost by the incident particle follows the Bethe-Bloch equation 1.19. This energy is transferred primarily to atomic electrons in the medium, which excite and may even overcome the binding energy of the shell. This ionisation process results in a free electron and an argon ion. For an incident ionising particle, $R$, the formula is

$$
R+A r \longrightarrow e^{-}+A r^{+}+R^{\prime}
$$

Additionally, the particle may raise an electron to a higher energy state via

$$
R+A r \longrightarrow A r^{*}+R^{\prime}
$$

or through the recombination of free electrons with argon ions,

$$
e^{-}+A r^{+} \longrightarrow A r^{*}
$$

These excited states combine with neutral argon atoms creating excited dimers, which radiate VUV photons upon deexitation:

$$
A r^{*}+A r \longrightarrow A r_{2}^{*} \longrightarrow 2 A r+h v_{a}
$$

or via the three atom interaction

$$
A r^{*}+2 A r \longrightarrow A r_{2}^{*}+A r \longrightarrow 3 A r+h v_{m}
$$

The argon dimer $A r_{2}^{*}$ can be excited into two main states, the singlet ${ }^{1}{ }^{1} \Sigma_{u}^{+}$, or the triplet ${ }^{2}$ ${ }^{3} \Sigma_{u}^{+}$, which decay with characteristic times $7 \mathrm{~ns}$ and $1.6 \mu \mathrm{s}$, respectively [57]. The excimer state energies of the two flavours are nearly equal, making the deexcitation photons essentially indistinguishable based on their spectra [67].

The mean energy required to ionise LAr is $23.6 \pm 0.3 \mathrm{eV}$ [53]. A minimally ionising particle (MIP), such as a cosmic muon, deposits on average $2.1 \mathrm{MeV}$ per $\mathrm{cm}$ of track length. However, not all of this energy is used to ionise charge. The ratio of mean energy per ion pair to the ionisation energy of LAr is about 1.6 [68], meaning about $60 \%$ of the energy deposited is converted to ionisation. The remaining energy goes to produce scintillation light via the production of various excited states and multiply ionised atoms, and a tiny

\footnotetext{
${ }^{1}$ Total spin angular momentum of 0 , such that there is only 1 allowed value for the spin component.

${ }^{2}$ Total spin angular momentum of 1 , such that there are 3 allowed values for the spin component.
} 
fraction is dissipated as heat. On top of this, further light is produced when electrons and ions recombine.

\subsubsection{Ionisation Electron Drift}

Under the influence of an applied electric field, free electrons in LAr drift $[69,70]$. The drift velocity $v_{D}$ is defined in terms of the mobility $\mu$ and the applied electric field $E$, by

$$
v_{D}=\mu E
$$

Collisions with nearby atoms prevent acceleration of the electrons to velocities higher than $v_{D}$, analogous to "terminal velocity". To a drifting charge carrier, LAr becomes like a viscous fluid [71]. In LAr, the mobility itself is dependent on the applied electric field and the temperature of the bulk LAr. Theory suggests [70] the temperature $(T)$ dependence on the drift velocity, and hence the mobility, is $v_{D} \propto T^{-3 / 2}$, which can account for about 3-5\% of the variations in the measurements of drift velocity between similar experiments [65]. A recent global fit [65] of previously published measurements parameterises the mobility in terms of the applied field (in $\mathrm{kV} / \mathrm{cm}$ ) and temperature,

$$
\mu(E, T)=\frac{a_{0}+a_{1} E+a_{2} E^{3 / 2}+a_{3} E^{5 / 2}}{1+\left(a_{1} / a_{0}\right) E+a_{4} E^{2}+a_{5} E^{3}}\left(\frac{T}{T_{0}}\right)^{-3 / 2},
$$

where $T_{0}=89 \mathrm{~K}$, and the global fit parameters are given by

$$
\begin{array}{lll}
a_{0}=551.6 \mathrm{~cm}^{2} / \mathrm{s} & a_{3}=4.29 \mathrm{~cm}^{9 / 2} / \mathrm{kV}^{5 / 2} \cdot \mathrm{s} \\
a_{1}=7953.7 \mathrm{~cm}^{3} / \mathrm{kV} \cdot \mathrm{s} & a_{4}=43.63 \mathrm{~cm}^{2} / \mathrm{kV}^{2} \\
a_{2}=4440.43 \mathrm{~cm}^{7 / 2} / \mathrm{kV}^{3 / 2} \cdot \mathrm{s} & a_{5}=0.2053 \mathrm{~cm}^{3} / \mathrm{kV}^{3}
\end{array}
$$

The measured drift velocities and mobilities from the literature $[48,50,72,73,74,75,76$, $77,78,79,80,81,82,83$ ], along with the global fit on mobility is shown in Figure 2.4.

\subsubsection{Free Electron Attachment to Impurities}

The most important factor which limits the performance of a LAr TPC is the attachment of free electrons to electronegative impurities. Free ionisation electrons in the LAr attach to electronegative impurity molecules (such as oxygen and water), reducing their drift velocity, so they no longer contribute to the signal, thereby reducing the total charge collected at the 

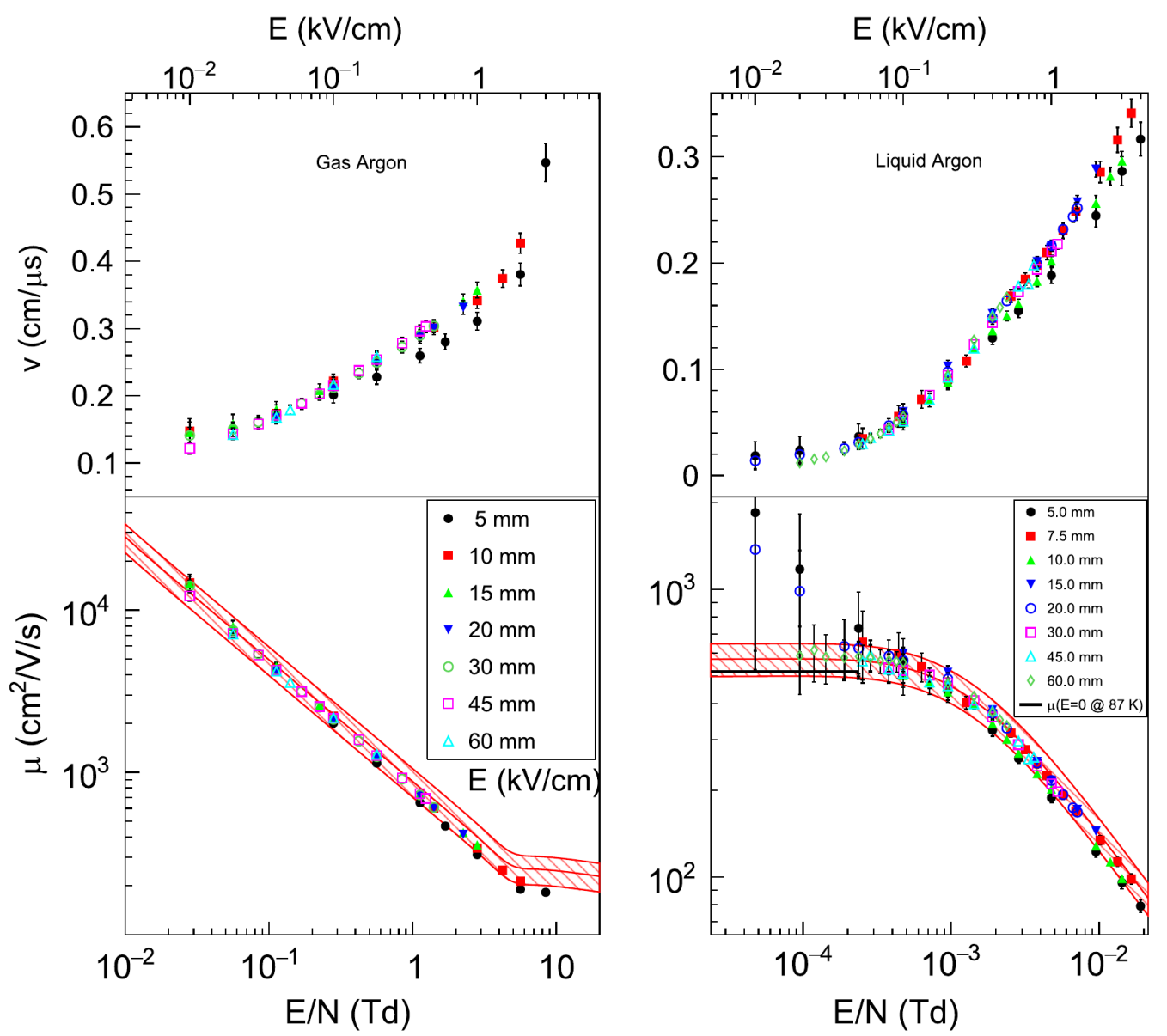

Fig. 2.4 Top: measured electron drift velocities. Bottom: electron mobility, shown with global fit (red band). Left: gaseous argon. Right: LAr. Measurements are shown for different drift distances. Top horizontal axis: applied electric field. Bottom horizontal axis: electric field divided by number density of argon atoms in units of $T d=10^{-17} \mathrm{~V} \mathrm{~cm}^{2}$. The expected mobility for zero field is shown as $518 \mathrm{~cm}^{2} / \mathrm{V} / \mathrm{s}$ [65]. 
anode. This reduction in drifting charge has a strong dependence on drift time and with the impurity species, in addition to the overall impurity concentration.

The attachment process occurs predominantly through the three body attachment reaction to impurity molecule $A B$ [84]:

$$
\begin{gathered}
e^{-}+A B \longleftrightarrow\left(A B^{-}\right)^{*} \\
\left(A B^{-}\right)^{*}+A r \longrightarrow A B^{-}+A r
\end{gathered}
$$

or by the dissociative reaction [35]

$$
e^{-}+A B \longrightarrow A+B^{-} \quad \text { or } \quad A^{-}+B
$$

The strengths of each of these reactions are largely dependent on the electron affinities of the atoms or molecules involved, and the energy of the electron [35].

The rate of electron attachment by impurities is given by [85]

$$
d[e] / d t=-k_{s}[S][e]
$$

where $[e]$ is the electron concentration, $[S]$ is the impurity concentration, and $k_{s}$ is the attachment rate constant for species $S$. Integration of this equation gives the exponential relationship for the time variation in electron concentration,

$$
[e(t)]=[e(0)] \exp \left(-k_{s}[S] t\right)=[e(0)] \exp \left(-t / \tau_{e}\right)
$$

The rate constant of electron attachment, $k_{s}$, is highly dependent on impurity species, $S$, and mildly dependent on applied electric field, as shown in Figure 2.5.

The lifetime parameter, $\tau_{e}$, of the exponential decay law in Equation 2.17 is related to the impurity attachment coefficients for each of the $i$ species:

$$
\tau_{e}=\left(\sum_{i} k_{i}\left[S_{i}\right]\right)^{-1}
$$

Some attachment coefficients have been measured for various impurity species, including $\mathrm{O}_{2}, \mathrm{CO}_{2}, \mathrm{SF}_{6}, \mathrm{~N}_{2}$, and $\mathrm{N}_{2} \mathrm{O}[85,86,87,88,89]$. Because most of these species exist in some concentration in commercial LAr, and are in some cases problematic to remove through purification, it is difficult to separate the effects of each individual impurity species, especially for extremely high purity $\operatorname{LAr}\left(\tau_{e}>1 \mathrm{~ms}\right)$. Therefore, it is common to quote the " $\mathrm{O}_{2}$-equivalent" impurity concentration, where oxygen here is chosen because it is a plentiful 
contaminant $(O(\mathrm{ppm})$ in commercial high purity LAr), and has a relatively high attachment coefficient. The reference value (i.e. widely used in estimating impurity concentrations) for the attachment constant for oxygen equivalent impurities, $k_{O_{2}}$, at $1 \mathrm{kV} / \mathrm{cm}$, is $5.5 \times 10^{10} \mathrm{M}^{-1} \mathrm{~s}^{-1}$ $\left(\sim 1.9 \mathrm{ppm}^{-1} \mu \mathrm{s}^{-1}\right)[85]$.

The electric field dependence of the attachment rate coefficient in LAr can be approximated by the empirical scaling relation [90]

$$
k_{O_{2}}=0.95 E^{-0.8}
$$

for electric field $E$ in $\mathrm{kV} \mathrm{cm}^{-1}$. Higher order dependencies of the attachment rate coefficient include the LAr temperature and additional electron attachment processes, such as neutralisation of electrons at the anode.

\subsubsection{Positive Space Charge}

Compared with the electron mobility in LAr of $518 \mathrm{~cm}^{2} \mathrm{~V}^{-1} \mathrm{~s}^{-1}$, the mobility of positive argon atoms in LAr is $6.61 \times 10^{-4} \mathrm{~cm}^{2} \mathrm{~V}^{-1} \mathrm{~s}^{-1}$, approximately six orders of magnitude lower. Therefore, on the electron drift time scale of a TPC, the ions can be considered as stationary. These positive ions may accumulate, given a high flux of ionising particles, distort the electric field as a whole, and attach to drifting electrons from future events. These effects are important in detectors with a high event rate, such as in particle colliders and detectors which are unshielded from cosmic rays.

The effect of space charge on the electric field in a TPC is derived in [91] to be

$$
E(x)=E_{0} \sqrt{\left(\frac{E_{A}}{E_{0}}\right)^{2}+\alpha \frac{x^{2}}{D^{2}}},
$$

where

$$
\alpha=\frac{D}{E_{0}} \sqrt{\frac{K}{\varepsilon \mu}},
$$

$E_{A}$ is the distorted field at the anode, $E_{0}=V / D$ is the electric field in the absence of space charge, $V$ is the voltage between anode and cathode, $D$ is the anode-cathode distance, $\varepsilon$ is the dielectric constant of the medium, $\mu$ is the positive ion mobility, and $K$ is the volume rate of creation of ion pairs. For values of $0 \leq \alpha<2$, the distorted field at the anode, normalised to the nominal field, can be solved numerically (see Figure 2.6). For the DUNE 35-ton TPC (see Chapter 3), $\alpha=0.789$, indicating a distorted normalised field at the anode as shown in 


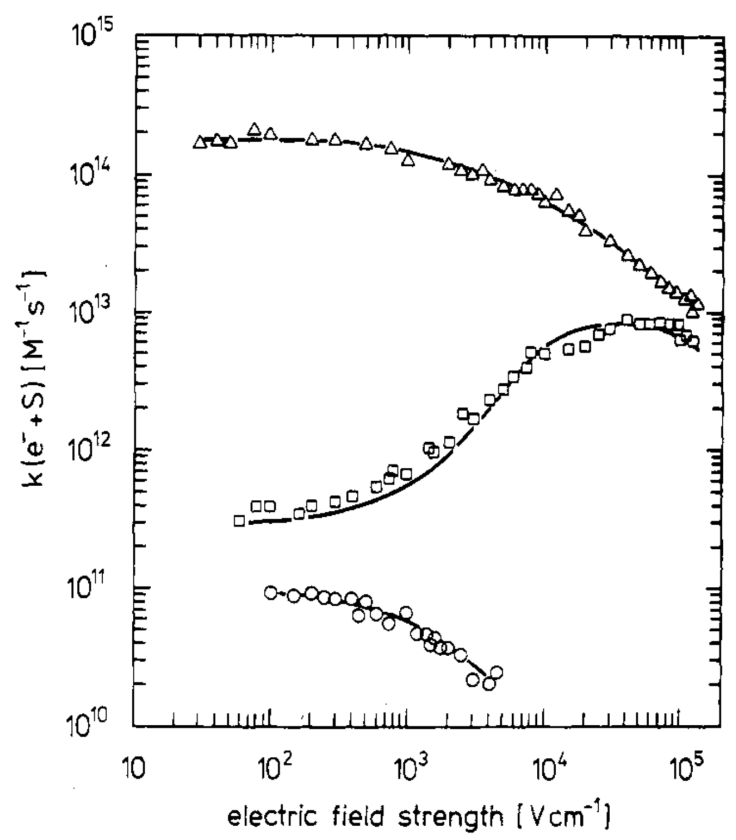

Fig. 2.5 Electron attachment rate constant in $\mathrm{LAr}_{\text {to }} \mathrm{SF}_{6}(\triangle), \mathrm{N}_{2} \mathrm{O}(\square)$, and $\mathrm{O}_{2}(\circ)$ [85].

Figure 2.7, decreasing by $11 \%$ from the nominal field at the anode (drift distance of $0 \mathrm{~cm}$ ) and increasing by $19 \%$ at the cathode (drift distance of $222 \mathrm{~cm}$ ).

\subsubsection{Electron Diffusion}

Electrons in a cluster of ionisation charge in LAr undergo Brownian motion as their energies are not high enough to cause further ionisation (i.e. they are "thermalised"). This motion causes the clusters of drifting electrons to diffuse between the point of ionisation and the anode readout (see Figure 2.8). Electron diffusion, therefore, impacts the operation of a TPC by reducing the position resolution of the interaction location, as the charge may be spread out over multiple electrodes in the anode. Also, the cluster diffuses along the drift direction, so the pulsed current induced in the anode spreads out in time.

Usually, the density of electrons in an ionisation cluster is too low for Coulomb repulsion to take place between electrons, so the cluster diffuses according to the Einstein-Smoluchowski relation of kinetic theory [92, 93]:

$$
D=\frac{\mu k T}{e}
$$

where $D$ is the diffusion coefficient with units of [Distance $]^{2}[\text { Time }]^{-1}, k T=0.0075 \mathrm{eV}$ is the electron temperature in LAr, $e$ and $\mu$ are the electron charge and mobility, respectively. 


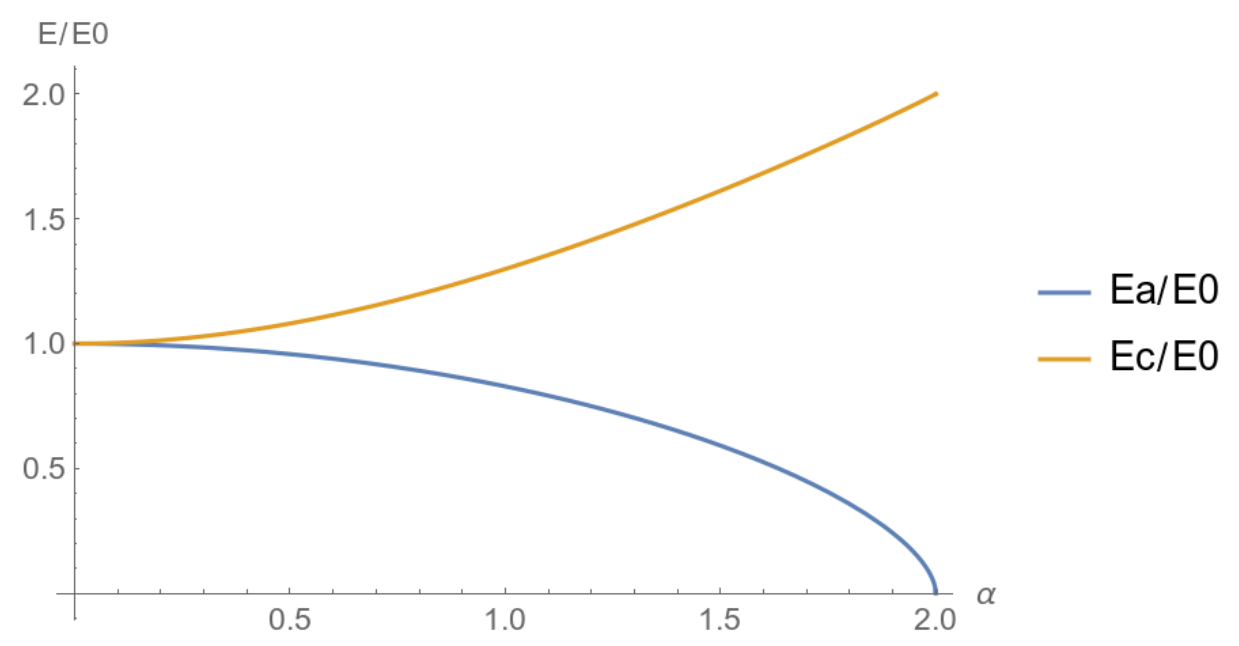

Fig. 2.6 Space charge distorted electric field at the anode (Ea/E0) and cathode (Ec/E0), as a function of $\alpha$.

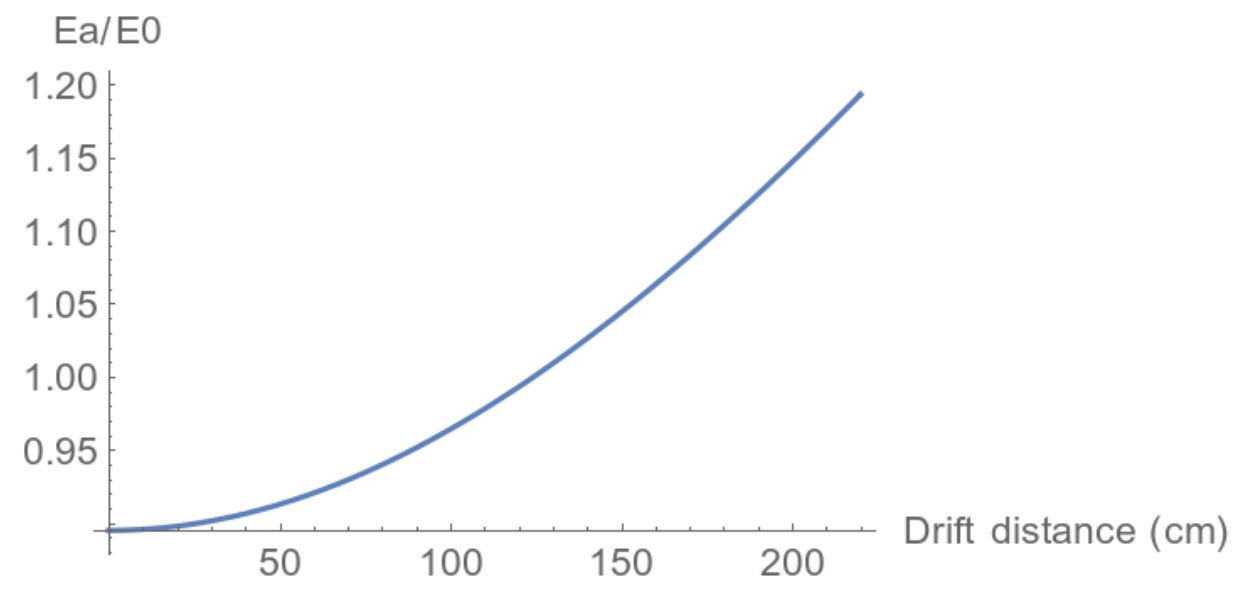

Fig. 2.7 Space charge distorted field at the anode for the DUNE 35-ton TPC. 


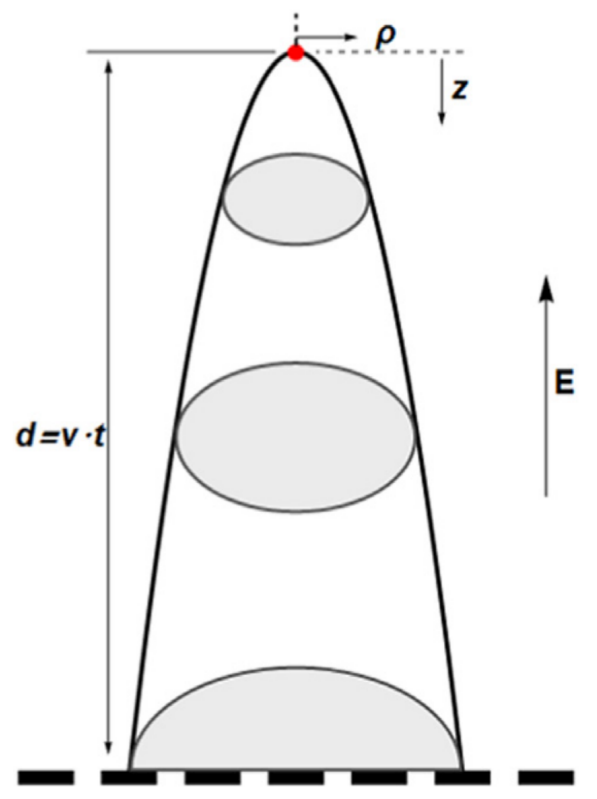

Fig. 2.8 Illustration of the diffusion process starting from a point source (top) and drifting across a distance $d$ in an electric field $E$ [65].

Because the size of the charge cluster increases over time, the resolution of reconstructing the original location of ionisation worsens with drift time.

Since $\vec{\mu}$ depends on the electric field applied $\vec{E}$ (Section 2.2.2), the diffusion of electrons behaves differently in directions parallel and perpendicular to the drift. This behaviour is described by Fick's equation:

$$
\frac{\partial n}{\partial t}=D_{L} \frac{\partial^{2} n}{\partial z^{2}}+D_{T}\left(\frac{\partial^{2} n}{\partial x^{2}}+\frac{\partial^{2} n}{\partial y^{2}}\right)-v \frac{\partial n}{\partial z}-\lambda v n
$$

which predicts an electron distribution function which is a 3D Gaussian [65]

$$
n(\rho, z, t)=\frac{n_{0}}{4 \pi D_{T} t \sqrt{4 \pi D_{L} t}} \exp \left(-\frac{(z-v t)^{2}}{4 D_{L} t}-\lambda v t\right) \exp \left(-\frac{\rho^{2}}{4 D_{T} t}\right)
$$

where $\rho^{2}=x^{2}+y^{2}$ is the coordinate perpendicular to $\vec{E}, z$ is the drift distance and is parallel to the direction of $\vec{E}, \lambda^{-1}$ is the mean free path taking into account the electron lifetime, $v$ is the drift velocity along $\vec{E}, D_{L}$ is the longitudinal diffusion coefficient (parallel to $\vec{E}$ ), and $D_{T}$ is the transverse diffusion coefficient (perpendicular to $\vec{E}$ ). The coefficients of diffusion then 
become:

$$
\begin{aligned}
& D_{T}=\frac{\mu k T}{e} \\
& D_{L}=\frac{k T}{e}\left(\mu+E \frac{\partial \mu}{\partial E}\right) \approx \frac{\mu \epsilon_{L}}{e},
\end{aligned}
$$

where $\epsilon_{L}$ is the "effective electron energy" at field $E$.

There are few experimental measurements of the diffusion coefficients, $D_{L}[65,94]$ and $D_{T}[95,96]$. The global fit on all measurements of $\epsilon_{L}$ is shown in Figure 2.9, and is parameterised according to

$$
\epsilon_{L}=\frac{b_{0}+b_{1} E+b_{2} E^{2}}{1+\left(b_{1} / b_{0}\right) E+b_{3} E^{2}}\left(\frac{T}{T_{1}}\right)
$$

where $T_{1}=87 \mathrm{~K}$ and best fit parameters are

$$
\begin{array}{ll}
b_{0}=0.0075 \mathrm{eV} & b_{1}=742.9 \mathrm{eV} \cdot \mathrm{cm} / \mathrm{kV} \\
b_{2}=3269.6 \mathrm{eV} \cdot \mathrm{cm}^{2} / \mathrm{kV}^{2} & b_{3}=31678.2 \mathrm{~cm}^{2} / \mathrm{kV}^{2} .
\end{array}
$$

The longitudinal diffusion coefficient is calculated by combining Equations 2.9, 2.27, and 2.26. At an electric field of $500 \mathrm{~V} / \mathrm{cm}, D_{L}=7.2 \mathrm{~cm}^{2} \mathrm{~s}^{-1}$ and, by extrapolation of existing data, $D_{T}=12 \mathrm{~cm}^{2} \mathrm{~s}^{-1}[65]$.

\subsubsection{Recombination}

While the ionisation electron yield of a charged particle interaction may be large, the total amount of free charge available for detection is significantly reduced. This is because a portion of the thermalised ionisation electrons recombine with positively charged ions near the point of ionisation. Recombination is, therefore, an undesirable property in a TPC as it reduces the observable signal size. The recombination probability depends on the applied electric field to quickly separate the positive ions from the electrons. Therefore, the effect can be reduced by applying larger fields. Recombination is a crucial factor in the event reconstruction and calibration process as it affects the calculation of the energy loss function of the incident particle, which determines the particle identification.

Onsager proposed [97] that the ionised electrons thermalise very close to the parent ion and recombine, within a sphere of influence defined by the Coulomb attraction. However, because the average ion-ion separation distance is much smaller than the average electron-ion separation distance, this type of recombination is disfavoured [35]. Jaffe proposed [98], 


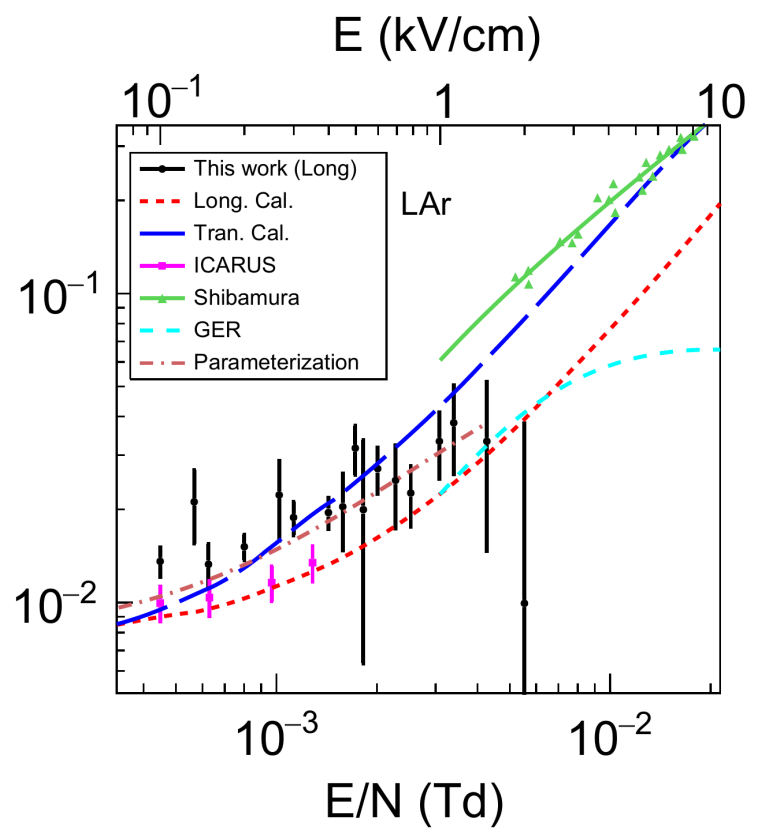

Fig. 2.9 Global fit on electron energy, $\epsilon_{L}$, for LAr [65]. Top horizontal axis: electric field. Bottom horizontal axis: electric field divided by number density of argon atoms in units of $T d=10^{-17} \mathrm{~V} \mathrm{~cm}^{2}$.

rather, that recombination depends on the overall density of electrons and ions along the ionisation trajectory, known as "columnar recombination". Assuming a Gaussian distribution of charges and equal electron and ion mobilities, the fraction of ionisation charge which survives recombination is given by

$$
\mathcal{R}_{J}=\left[1+\frac{\alpha N_{0}}{8 \pi D} \sqrt{\frac{\pi}{z^{\prime}}} S\left(z^{\prime}\right)\right]^{-1}, \quad z^{\prime}=\frac{2 r_{0}^{2} \mu^{2} E^{2} \sin ^{2} \phi}{\pi D^{2}}
$$

where $\alpha$ is a coefficient related to recombination, $D / \mu$ is the Einstein-Smoluchowski relation for diffusion [92, 93], $E$ is the electric field, $\phi$ is the angle between the electric field and the particle direction, $N_{0}$ is the linear density of electron-ion pairs, $r_{0}$ is the average electronion separation distance, and $S\left(z^{\prime}\right)$ defines the angular dependence in $z^{\prime}$. Equation 2.30 is commonly referred to as "Birks' model".

Since the mobility of electrons is orders of magnitude greater than the mobility of positive ions, the formulation of the recombination process can be simplified [99] by assuming negligible electron diffusion and ion mobility in LAr. This so-called "Box model" is given by

$$
\mathcal{R}_{\mathrm{Box}}=\frac{1}{\zeta} \ln (\alpha+\zeta) \text { where } \zeta=k_{\mathrm{Box}} N_{0} / 4 a^{2} \mu E,
$$


where $N_{0} / 4 a^{2}$ represents the charge density in a box of size $a, \alpha$ is a fit parameter, and $k_{\mathrm{Box}}$ is a constant.

None of these models accurately reflect all experimental data. For example, the data of an early large LAr TPC experiment are well described by Jaffe theory [100], whereas the box model, modified to remove particular experimental running conditions, best describes the data of a recent LAr TPC at high ionisation density [101]. Further study is required to understand fully and model recombination for a broader range of electric fields and ionisation charge densities. A comparison of three models of recombination, each fit to the same data set, over a range of $d E / d x$ is shown in Figure 2.10. Note the difference in model predictions for an energy loss of $2.1 \mathrm{MeV} / \mathrm{cm}$ (MIP in LAr).

\subsubsection{Energy Resolution}

While the energy required to produce an electron-ion pair, or a photon, is well defined (see Table 2.1), statistical fluctuations in the number of electrons or photons produced causes uncertainty in determining the actual energy deposited in the LAr. These fluctuations are described by the Fano factor [102], which is defined as the degree to which the fluctuations deviate from the ideal Poisson distribution of number of electron-ion pairs produced. For LAr, the theoretical value is 0.107 [103], giving a maximum intrinsic energy resolution of $0.377 \%$ for a $1 \mathrm{MeV}$ ionising electron [104].

To date, the best-measured energy resolution in LAr is $2.7 \%$ (26 keV for a $976 \mathrm{keV}$ electron ionisation) [105]. Fluctuations in the number of $\delta$ electrons along an ionisation track (see Section 2.2.8.2) explain the loss of resolution as they have higher ionisation densities and, therefore, are less likely to survive recombination [106].

\subsubsection{Background Processes}

In addition to the charge transport properties of a LAr TPC, there are other physical processes which distort the event image projection and reconstruction. While the influence of recombination, attachment to impurities, space charge, and diffusion can be mitigated by changing the experimental conditions (e.g. increasing electric field, purification), certain background processes are inherent to LAr, and their effects cannot be avoided. For the two processes discussed below, the end product is the same-a spontaneously produced energetic electron—and, as a result, the energy resolution of the detector is reduced. 


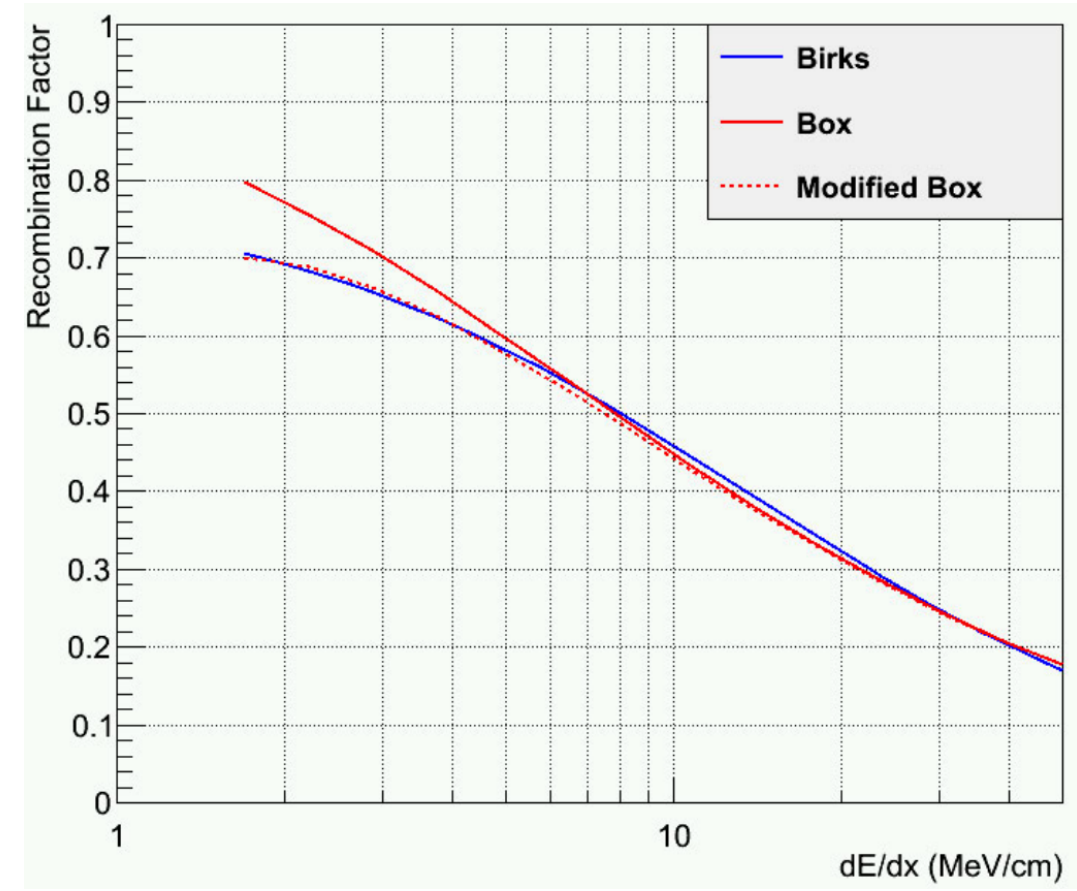

Fig. 2.10 Comparison of best fit recombination coefficients for three models using the same underlying data for each (data not shown).

\subsubsection{1 ${ }^{39}$ Ar Decay}

The decay of a naturally occurring unstable argon isotope is a significant background process in a LAr TPC for low energy, rare physics events such as dark matter interactions and supernova neutrino detection. The significance is not only because of the high event rate in a large detector but because the interactions of the daughter particles may mimic, for example, the observable scintillation signal from a low energy neutrino elastic scattering event [107].

The main isotopes of natural argon are ${ }^{40} \mathrm{Ar}(99.6 \%),{ }^{36} \mathrm{Ar}(0.33 \%)$, and ${ }^{38} \mathrm{Ar}(0.06 \%)$, which are all stable. However, because argon is produced by the distillation of liquefied air, and because of cosmic ray spallation, a long-lived unstable isotope, ${ }^{39} \mathrm{Ar}$, is produced via the $(n, 2 n)$ reaction. It is a $\beta^{-}$emitter with half-life of 269 years and $Q$ value of $565 \mathrm{keV} \mathrm{[108].}$ In atmospheric argon, the activity of ${ }^{39} \mathrm{Ar}$ is found to be $1 \mathrm{~Bq} \mathrm{~kg}^{-1}$ [107]. For the next generation LAr TPC experiment with total LAr mass of $O$ (kilotons), the resulting trigger rate from ${ }^{39} \mathrm{Ar}$ is a significant concern for low energy, rare physics event detection.

\subsubsection{Delta Electron Emission}

Until this point, the presented models of recombination and energy resolution assumed that ionising radiation creates a uniform density of charge along the particle track. However, 
the kinetic energy transferred to ionisation electrons is not a constant, and sometimes the electron can become energetic enough to cause secondary ionisation and scintillation. These randomly emitted electrons are commonly called "delta" (or just $\delta$ ) electrons.

Studies have shown that delta production can cause degradation in experimental energy resolution in LAr [109]. By considering a two-component coefficient (for primary and secondary ionisation) for certain particle interaction events, they were better able to explain inconsistencies between recombination data and theory.

Depending on the kinetic energy transferred, the track length of delta electrons can range from zero up to several tens of centimetres. As reported in [110], an example of an ionising muon track is shown in Figure 2.11 with several delta electron tracks emanating from random points along the track, travelling some distance, then thermalising. Excellent spatial resolution of detection of ionisation events can help to reduce the effects of delta electrons on energy resolution and recombination rates. For example, an experiment [111] which utilised electrostatic focusing was able to efficiently separate primary ionisation charge signals from secondary delta electrons, and measure their energy distribution.

\subsubsection{Particle Detection}

As given in Table 2.1, the radiation length of LAr is $14 \mathrm{~cm}$, and the nuclear interaction length is $86 \mathrm{~cm}$. Therefore relatively small volumes of LAr are excellent at stopping and absorbing radiation. The energy absorbed is proportionally and efficiently converted into ionisation charge and scintillation light as described in the sections above. Because of this, LAr is an exceptional calorimeter. Also, because diffusion is low and high electron velocities can easily be attained, good vertex resolution is possible, so LAr can also perform well as a high-resolution imaging medium given an appropriate detector design. In general, because of the low ionisation energy of LAr, the ionisation electron yield is high. Therefore, because different interacting particles have different energy loss functions, identification of particle species is possible by analysing directly the $d E / d x$ of the track (for example, [112]). Furthermore, multiplication of electrons is not strictly necessary to read out the signals from particle interactions which ionise the LAr. The scintillation light yield of LAr is also high, which allows simultaneous triggering of events in the detector while still leaving sufficient ionisation charge for inducing measurable currents in the readout instruments.

Due to its high $Z$ and high density, LAr is an excellent detection medium for various scattering, nuclear, or electromagnetic interactions between argon nuclei (or their electrons) and incident particles. The benefits of using LAr as a detection medium are numerous, and the drawbacks significant but surmountable. 

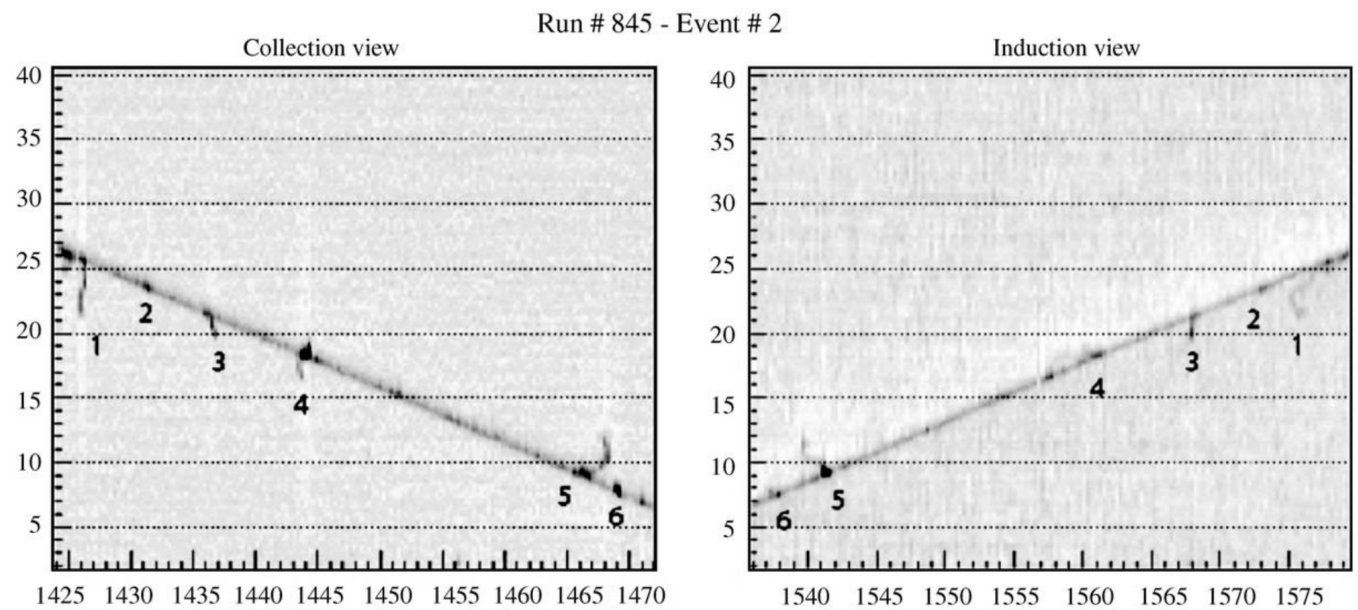

Fig. 2.11 Example event display showing ionising muon track with several delta electron tracks emanating from the primary particle, from two different views [110].

\subsection{Past, Current, and Future LAr TPCs and Their Appli- cations}

Since 1977, several LAr TPCs have been constructed and successfully operated, including prototypes for technology research and development as well as for physics experiments. Since the success of the first major large-scale LAr TPC, many current and future experiments are enthusiastically pursued. Much effort is being invested in technology development and construction which hints at the confidence that the scientific and funding communities have of the LAr TPC idea. It is seen as one of the best hopes for unravelling the remaining mysteries of the nature of neutrinos, dark matter, proton decay, and even the astrophysics of supernovae.

Single and double phase LAr TPCs detect particles in much the same manner. However, many of the engineering requirements to build a large-scale TPC differ between the two technologies. Each has benefits and drawbacks, but both are considered feasible options for the future of massive LAr TPCs for neutrino and other rare physics experiments.

\subsubsection{Past LAr TPCs}

In addition to many prototype LAr TPCs built around the world since the late 1970s, two main experiments stand out as they provided various neutrino physics results, as well as valuable technological progress toward the detector technology itself. Both of the experiments described here have had many years of successful running, have given many insights into neutrino interactions in LAr, and have demonstrated the feasibility of LAr TPCs for neutrino research. 


\subsubsection{ICARUS}

As mentioned above, the ICARUS T600 (Imaging Cosmic And Rare Underground Signals) [49] was the first large-scale operational LAr TPC instrumental in the development of the technology as a whole (see Figure 2.12). The TPC contained 476 active tons of LAr and was built deep underground at the Gran Sasso underground laboratory. It was rectangular in design and utilised a central cathode and two outer wire readout anodes. Each anode contained three planes of wires at $60^{\circ}$ from each other. In total, 55000 readout channels were instrumented for simultaneous readout. The TPC used a drift field of $500 \mathrm{~V} / \mathrm{cm}$, had a maximum drift length of $1.5 \mathrm{~m}$, and measured a maximum electron lifetime of $16 \mathrm{~ms}$ corresponding to a charge attenuation of $6 \%$ over the full $1.5 \mathrm{~m}$ drift [100]. An array of 74 photomultiplier tubes coated in tetraphenyl butadiene (TPB) was installed in the cryostat to measure scintillation light and to trigger events.

The T600 was placed in the CERN Neutrinos to Gran Sasso (CNGS) accelerator neutrino beam to measure neutrino oscillations at a baseline of $730 \mathrm{~km}$ with energies peaked around 10-30 GeV [113]. The experiment has published many results including:

- Experimental observation of an extremely high electron lifetime [114]

- Study of electron recombination in liquid argon [100]

- Precise 3D track reconstruction algorithm [115]

- The trigger system of the ICARUS experiment for the CNGS beam [116]

- Precision measurement of the neutrino velocity with the ICARUS detector in the CNGS beam [117]

- Search for anomalies in the $v_{e}$ appearance from a $v_{\mu}$ beam [113]

- Observation of long ionising tracks [110]

The detector finished running in 2013, was partially dismantled, refurbished, and has been shipped to Fermilab to be part of the short baseline neutrino programme (Section 2.3.2.2) to help understand the LSND [16] and MiniBooNE [17] neutrino oscillation results.

\subsubsection{ArgoNeuT}

ArgoNeuT [118] was a small ( $\sim 170$ L) LAr TPC at Fermilab whose main purpose was to measure various neutrino-nucleus interaction properties, such as neutrino-argon cross sections. The TPC was located in the Neutrinos at the Main Injector (NuMI) beamline near the MINOS experiment near detector and, over the course of the run, measured around 9000 charged current neutrino interactions [118]. The experiment has successfully measured neutrino-argon 


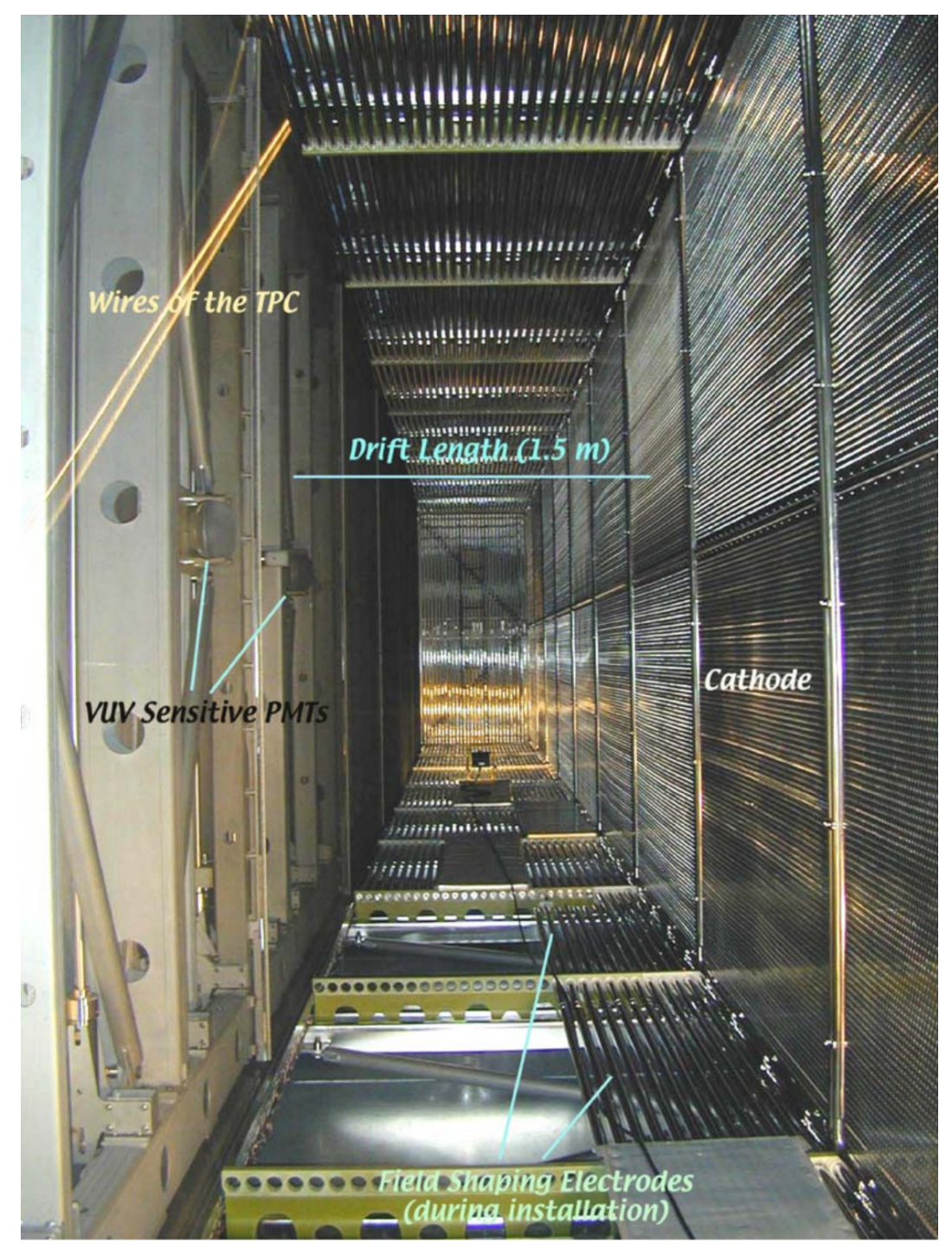

Fig. 2.12 Photo of a slice of the ICARUS detector showing anode wire planes (left), central cathode (right), field shaping rings, and VUV sensitive PMT [49]. 
cross sections [119, 120], neutrino-nuclear interaction parameters [121, 122, 123], as well as electron-ion recombination parameters in LAr [101]. An example neutrino event display is shown in Figure 2.13.

ArgoNeuT is an important milestone as it was the first LAr TPC in a neutrino beam in the United States. Information learned in this experiment has impacted the rest of Fermilab's LAr TPC development programme. In addition to the physics understanding gained by the experiment, the automated event processing and reconstruction developed by the experiment has morphed into what is now LArSoft [124], currently used by all other Fermilab-based LAr TPC experiments. Since the end of the ArgoNeuT run, the cryostat and detector have been repurposed into the LArIAT [125] experiment whose primary purpose is to study further aspects necessary for a large scale high precision neutrino detector. These aspects include studying particle identification, calibration of energy-to-charge conversion in LAr, and the signal generation and processing in LAr TPCs in general.

\subsubsection{Current and Future LAr TPCs}

Building on the success of ICARUS and ArgoNeuT/LArIAT, the current efforts toward single phase LAr TPC development are mainly focused on MicroBooNE and the Fermilab Short Baseline Neutrino (SBN) programme.

\subsubsection{MicroBooNE}

MicroBooNE (see Figure 2.14) is a currently running LAr TPC at Fermilab, which was built to investigate the short baseline neutrino oscillation anomalies of LSND [16] and MiniBooNE [17], as well as measure neutrino-argon cross sections, and further the development of technologies crucial in operating LAr TPCs such as reconstruction techniques. The detector contains 90 active tons of LAr, with a drift length of about $2.5 \mathrm{~m}$, and design applied electric field of $500 \mathrm{~V} / \mathrm{cm}$. The cryostat contains 32 TPB coated PMTs for scintillation light collection and triggering. Additionally, the detector utilises a calibration method whereby a $266 \mathrm{~nm}$ laser creates a straight line of ionisation in LAr to investigate space charge in the TPC. The detector is placed in the Booster Neutrino Beam (BNB) at Fermilab, approximately $500 \mathrm{~m}$ from the neutrino production target. The charge readout technology is almost geometrically identical to that of ICARUS T600: two induction planes of wires and one collection wire plane, at $60^{\circ}$ from each other, and with a plane-to-plane spacing of $3 \mathrm{~mm}$. MicroBooNE is different in that it utilises front-end readout electronics which operate submerged in the LAr. 

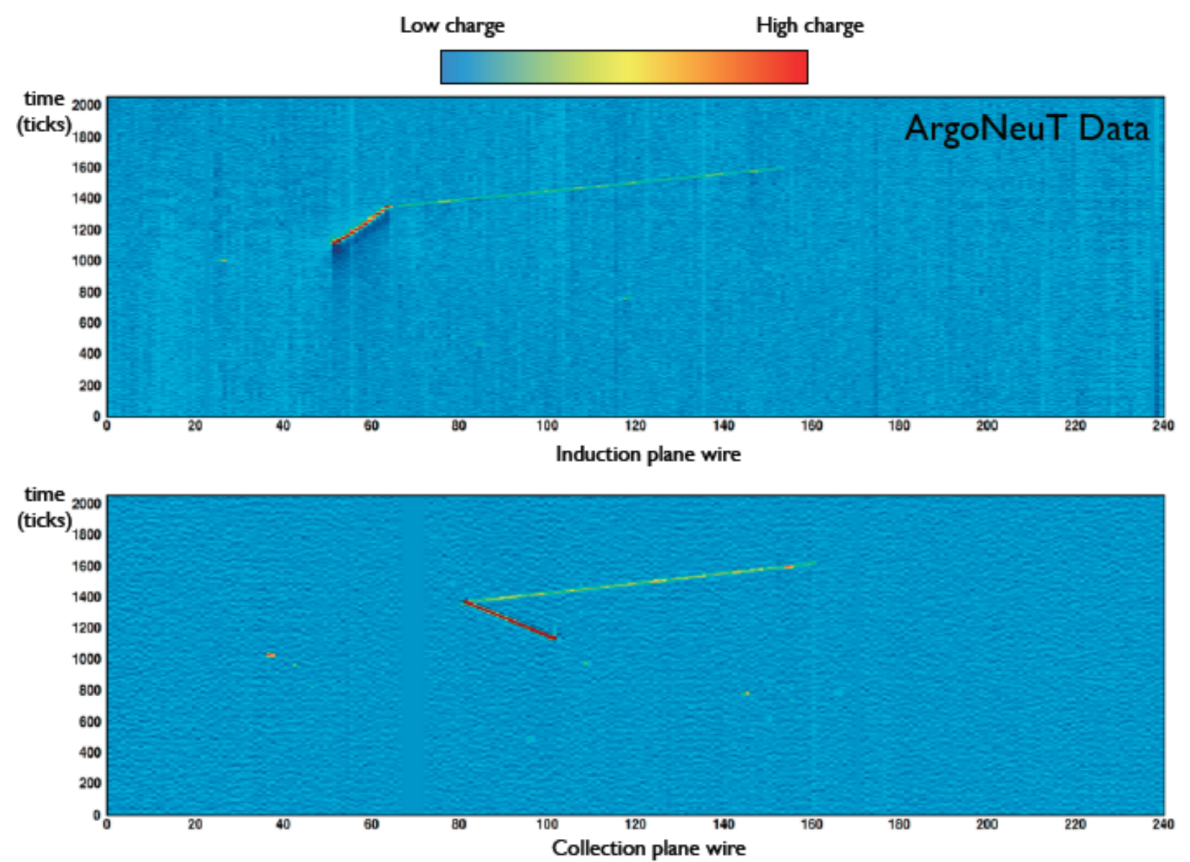

Fig. 2.13 A charged current $v_{\mu}$ event candidate from the ArgoNeuT LAr TPC [118].

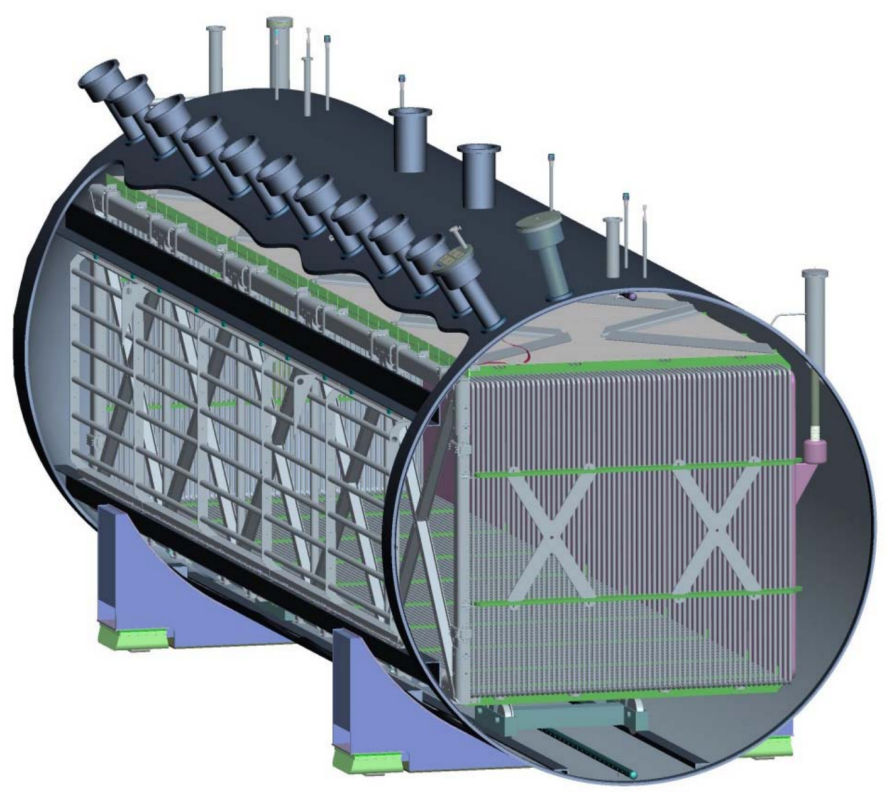

Fig. 2.14 Schematic of the MicroBooNE cryostat and TPC, showing major elements including field shaping rings, PMT mounting rack (left side, frame with horizontal bars), and anode wiring frame (left side of cryostat, frame supported by " $X$ " cross beams) [32]. 
The MicroBooNE detector began operation in summer 2015 and continues to measure particle interactions in LAr. Already, results have been published in many areas regarding the operation and reconstruction of events in the TPC, for example, [126, 127].

\subsubsection{SBN}

The Short Baseline Neutrino ( $\mathrm{SBN}$ ) programme is a planned three detector experiment which intends to explain the MiniBooNE/LSND anomaly by testing the "sterile" neutrino hypothesis. Of the three detectors, two have already been discussed: ICARUS T600 and MicroBooNE. As mentioned, the refurbished ICARUS T600 detector will be installed about $600 \mathrm{~m}$ from the neutrino production target in the BNB at Fermilab, and MicroBooNE will be operated in its current location with a baseline of approximately $500 \mathrm{~m}$. A third LAr TPC, called Short Baseline Neutrino Detector (SBND) [19] will operate about $110 \mathrm{~m}$ from the neutrino production target in the same beamline. SBND is currently being constructed and will have an active LAr mass of around 112 tons and a drift distance of approximately two metres. It will also have VUV light collection as well as nearly full coverage by scintillating muon counters to tag and trigger on crossing muons. Operation of the SBND detector is scheduled to begin in 2019 .

\subsubsection{The Deep Underground Neutrino Experiment}

Much of the current global effort in building and understanding LAr TPCs is in preparation for running the Deep Underground Neutrino Experiment (DUNE) far detector [36], a massive LAr TPC to be located 4850 feet underground at the Homestake Mine in South Dakota. The far detector complex is illustrated in Figure 2.15. The experiment is anticipated to consist of more than 40 kilotons of instrumented ultrapure LAr, separated among several massive cryostats, each containing around ten kilotons of LAr. The construction of the full far detector complex will be staged, beginning with a $10 \mathrm{kt}$ single phase LAr TPC with wire-based readout, and continuing with similar but not necessarily identical LAr TPCs including possibly a dual phase detector. As shown in Figure 2.16, the detector will be located in a high intensity, high energy neutrino beam produced at Fermilab, $1300 \mathrm{~km}$ away. Two near detectors will be built about $1 \mathrm{~km}$ away from the neutrino production target to reduce neutrino flux systematic uncertainties: a LAr TPC and a scintillation tracking detector.

The DUNE far detector reference design consists of a massive segmented TPC, illustrated in Figure 2.17. Each segment, defined by the size of a single anode plane assembly (APA), is $2.3 \mathrm{~m}$ wide and $6 \mathrm{~m}$ tall and has a drift distance of $3.6 \mathrm{~m}$ to the nearest cathode plane assembly (CPA). The full $10 \mathrm{kt}$ TPC consists of $25 \times 4 \times 2$ of such segments. For the one-third of 


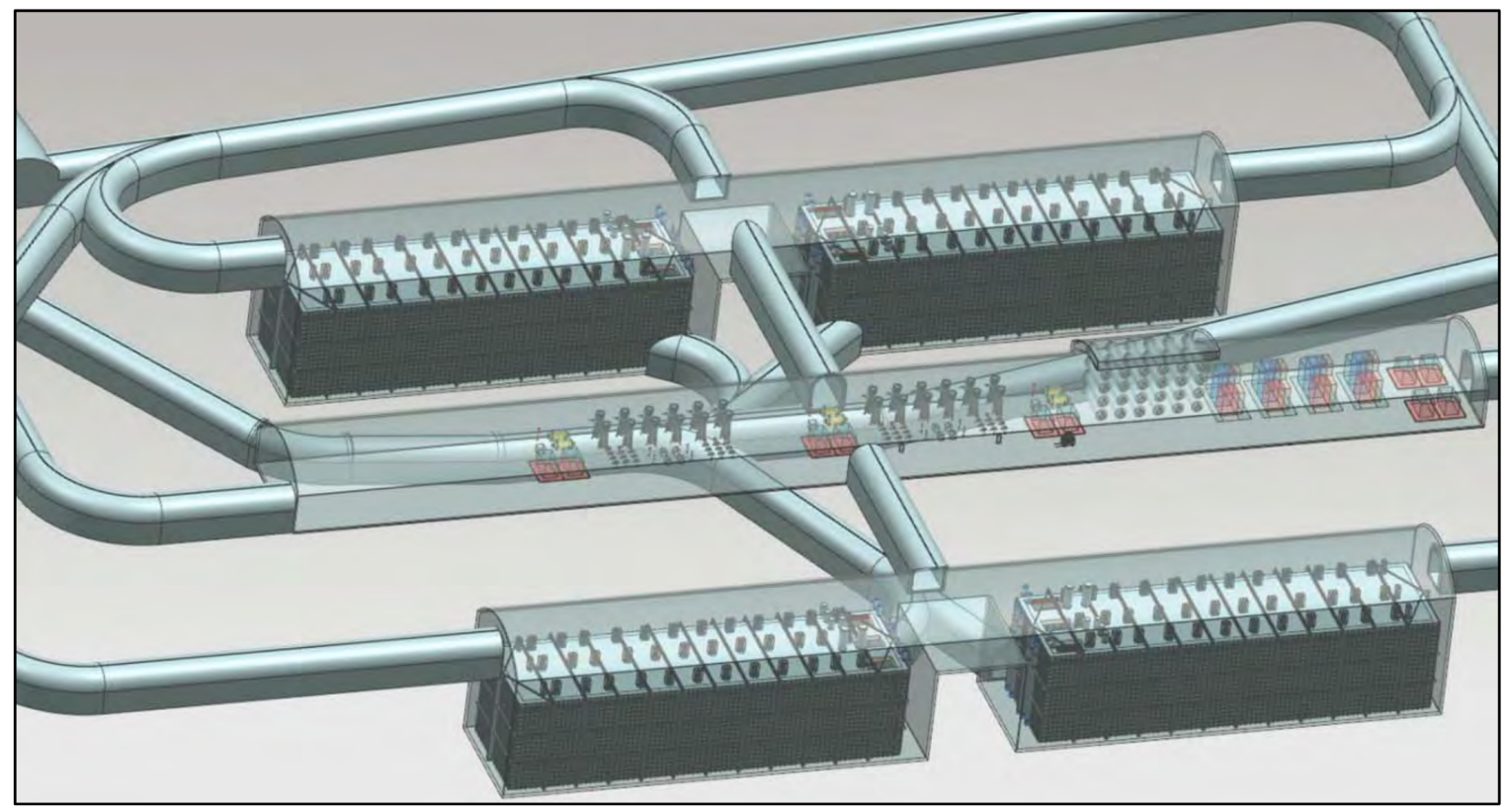

Fig. 2.15 Illustration of the DUNE underground complex, including four $10 \mathrm{kt}$ detector modules, and central service hall for cryogenics and electronics.

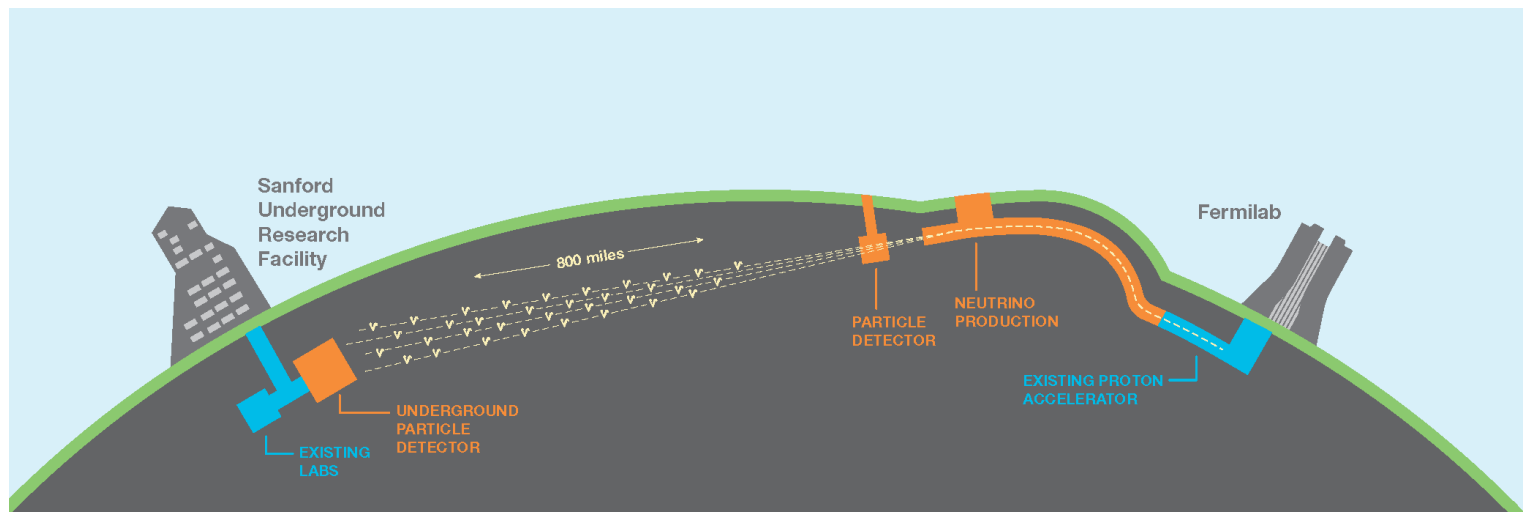

Fig. 2.16 The main DUNE detector components and schematic. 
APAs which are not adjacent to a wall, ionisation signals are simultaneously read out on both sides from two different drift volumes. A high voltage of $-180 \mathrm{kV}$ is applied to each CPA, providing a drift field of $500 \mathrm{~V} / \mathrm{cm}$.

Ionisation charge signals are read out by the TPC using induction and collection wires. In DUNE, four wire planes are used on each side of each APA: an uninstrumented grid to shield the wires from drifting ion charge signals, two collection planes at $\pm 35.7^{\circ}$ from the vertical, and one plane of collection wires at $0^{\circ}$ from the vertical. The interwire spacing in each plane and the interplane spacing are both about $4.7 \mathrm{~mm}$, which is expected to provide a position reconstruction sensitivity of about $(1.1,1.4,1.7) \mathrm{cm}$ in $(x, y, z)$. These and other relevant performance capabilities of the DUNE TPC design are summarised in Table 2.2.

To reduce the number of cryostat penetrations, and to keep the analogue signal path as short as possible, the amplification and digitisation of wire signals will be performed at LAr temperature, near the APAs. Another benefit of the cooling of the complementary metal oxide semiconductor (CMOS) components is the reduction of thermal noise in the semiconductors.

The DUNE far detector will contain a system of photon detectors to assist in the calorimetry of event reconstruction, as well as to provide event triggering via the scintillation light produced in ionisation events in the LAr. The photon detectors will be silicon photomultipliers (SiPM) with wavelength shifting waveguides to transform the VUV light closer to the sensitive region of the SiPMs.

It is intended for the DUNE far detector to utilise a detector calibration system based on ultraviolet lasers [128]. Such a system will be able to create straight "tracks" of ionisation at deliberate angles in the detector. Then the effects of electron lifetime, field non-uniformities, drift time, and even space charge can be calibrated very precisely.

Data acquisition (DAQ) in the DUNE far detector must accommodate all of the TPC readout, photon detector readout, calibrations, slow controls, and event timing. The DAQ system must have a high uptime and the capability to record events across the TPC elements for both low rate (e.g. beam neutrinos) and high rate (e.g. supernova burst neutrinos in the first couple of seconds following the explosion) events with little or no data compression. The DAQ must aggregate all detector component signals into a format readable offline during analysis.

In general, the scaling up of the LAr TPC to the kiloton scale has unknown consequences for the integral detector performance characteristics. Purification of such a large volume of LAr to achieve a $3 \mathrm{~ms}$ electron lifetime has never been attempted before and could present issues. Another untested factor at this stage is the long-term, low noise, functioning of cold electronics, which is currently being studied in a medium scale LAr TPC prototyping project, called protoDUNE [129]. This engineering prototype is meant to test, specifically, the issues 


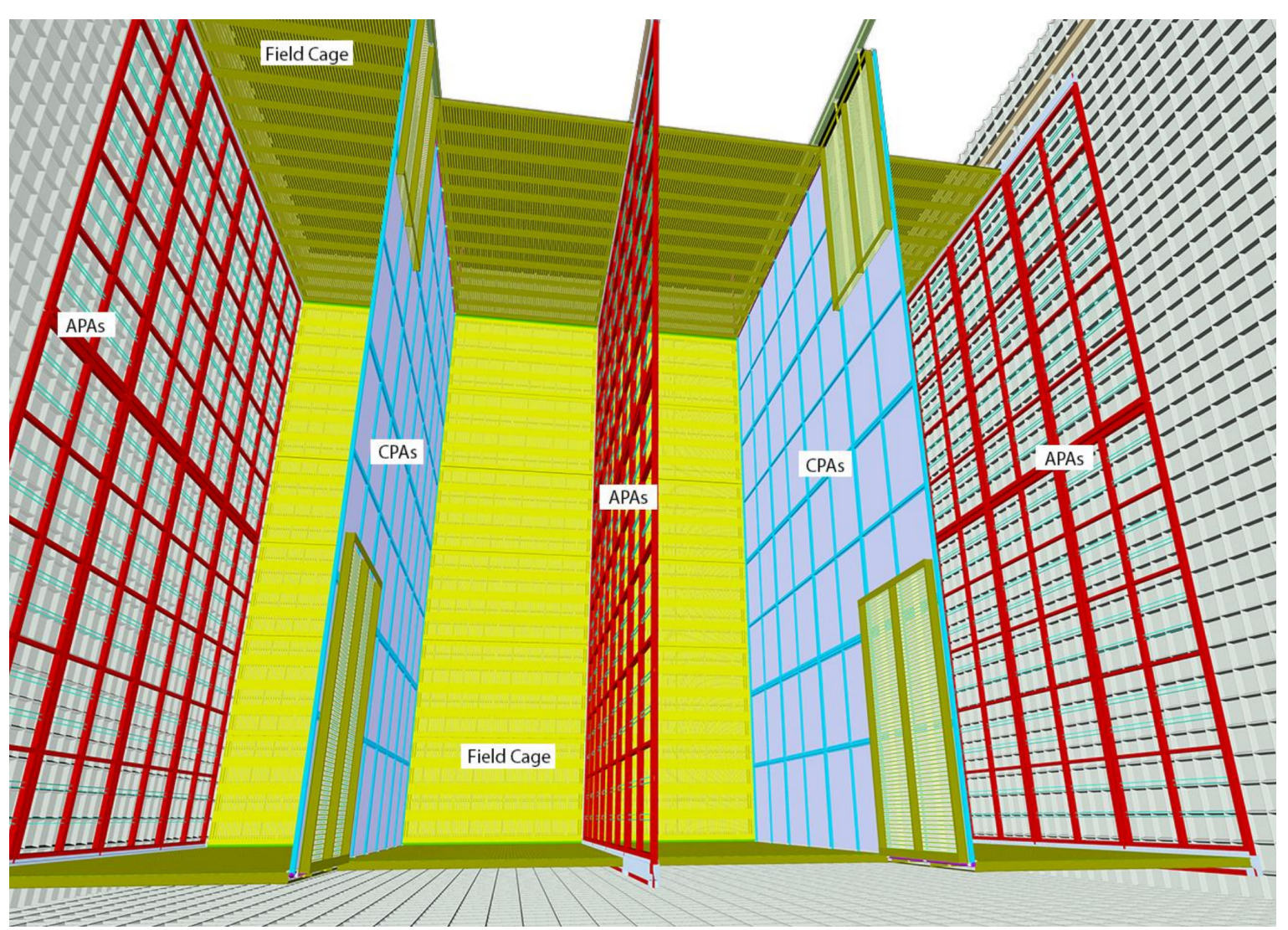

Fig. 2.17 Schematic of DUNE reference TPC design, showing APAs (red), CPAs (blue), and field shaping cage (green/yellow) [36]. 
Table 2.2 Summary of performance parameters for DUNE far detector [36]. References for "Achieved Elsewhere" values are in [36].

\begin{tabular}{llll}
\hline Parameter & Requirement & $\begin{array}{l}\text { Achieved } \\
\text { Elsewhere }\end{array}$ & $\begin{array}{l}\text { Expected } \\
\text { Performance }\end{array}$ \\
\hline Signal/Noise Ratio & $9: 1$ & $10: 1$ & $9: 1$ \\
\hline Electron Lifetime & $3 \mathrm{~ms}$ & $>15 \mathrm{~ms}$ & $>3 \mathrm{~ms}$ \\
\hline $\begin{array}{l}\text { Uncertainty on Charge } \\
\text { Loss due to Lifetime }\end{array}$ & $<5 \%$ & $<1 \%$ & $<1 \%$ \\
\hline $\begin{array}{l}\text { Dynamic Range of Hit } \\
\text { Charge Measurement }\end{array}$ & $15 \mathrm{MIP}$ & $15 \mathrm{MIP}$ \\
\hline Vertex Position Resolution & $(2.5,2.5,2.5) \mathrm{cm}$ & $(1.1,1.4,1.7) \mathrm{cm}$ \\
\hline $\begin{array}{l}e-\gamma \text { separation } \\
\text { efficiency }\end{array}$ & $>0.9$ & 0.9 \\
\hline $\begin{array}{l}e-\gamma \text { separation } \\
\gamma \text { rejection }\end{array}$ & $>0.9$ & 0.99 \\
\hline $\begin{array}{l}\text { Multiple Scattering Resolution } \\
\text { On Muon Momentum }\end{array}$ & $\sim 18 \%$ & $\sim 18 \%$ & $\sim 18 \%$ \\
\hline $\begin{array}{l}\text { Electron Energy Scale } \\
\text { Uncertainty }\end{array}$ & $\sim 5 \%$ & $\sim 2.2 \%$ & $>90 \%$ \\
\hline $\begin{array}{l}\text { Electron Energy Resolution } \\
\text { Energy Resolution for }\end{array}$ & $0.15 / \sqrt{E(\mathrm{MeV})}$ & $0.33 / \sqrt{E(\mathrm{MeV})}$ \\
Stopping Hadrons & $\oplus 1 \%$ & $10 \%$ & \\
\hline $\begin{array}{l}\text { Short Electron Track } \\
\text { Finding Efficiency }\end{array}$ & $>90 \%$ & & \\
\hline
\end{tabular}


related to scaling up the LAr TPC design in preparation for the construction and operation of the DUNE far detector.

Operation of the first DUNE far detector module is anticipated to begin in 2026, and the far detector complex is expected to reach an exposure of $120 \mathrm{kt}$ MW year by 2035 accounting for the staged construction of additional detector modules [36].

\subsubsection{Dual Phase Argon TPCs}

The main benefit of dual phase argon TPC technology is the ability to amplify the ionisation charge signal and create secondary scintillation light in the process. This is important especially for low energy event detection, such as nuclear recoils by hypothetical weakly interacting massive particle (WIMP) dark matter where transferred kinetic energy is in the range of 1-100 keV [130]. High-resolution tracking of charged particle interaction products for these rare low energy events helps to identify the physics and separate backgrounds [36].

Some examples of current dual phase argon TPCs include ArDM [41] and DarkSide [42]. The interesting events in these detectors will be recoils from WIMP scattering on argon nuclei. The ionisation from these recoiling charged particles is drifted in an electric field, extracted from the liquid into the vapour, and amplified by electron multiplication. In DarkSide, the amplified electrons are converted to light via electroluminescence and read out with PMTs. In ArDM, the secondary scintillation from the electron multiplication is read out with PMTs, in addition to reading out the charge directly with a segmented anode.

WA105 at CERN [34] is, so far, the largest dual phase argon TPC with charge amplification and $2 \mathrm{D}$ strip-based projective charge readout in the gas phase. The $3 \times 1 \times 1 \mathrm{~m} \mathrm{TPC}$ reads out the amplified charge directly in addition to the primary and secondary scintillation light. Multiplication of the electrons occurs using a Large Electron Multiplier (LEM) which causes the drifting charge to avalanche, creating more electrons due to secondary ionisation as the primary electrons gain kinetic energy from the high electric field. A diagram of this process is shown in Figure 2.18. WA105 is a prototype for the scaling up of dual phase argon TPC technology for neutrino detection-possibly as an alternate design for one or more DUNE far detector $10 \mathrm{kt}$ modules.

Development of low capacitance pixel-based anode charge readouts is actively pursued for two-phase LAr TPCs, as an alternative to wire-based readout (see Figure 2.19). This scheme has many benefits over the DUNE style wire-based readout. For example, both projected views are symmetric and unipolar. Also, their low capacitance allows greater channel density without necessarily increasing the number of overall channels, increasing the vertex resolution of the event. 


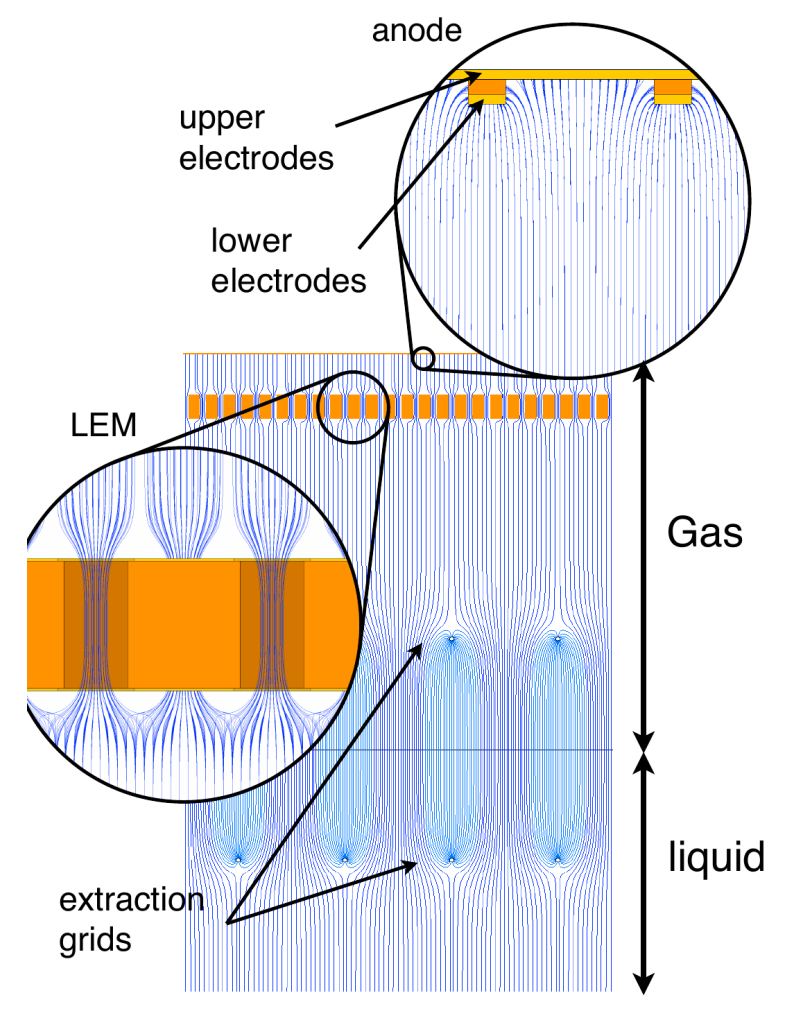

Fig. 2.18 Schematic of charge readout in the WA105 TPC [131]. The high density of field lines across the liquid-gas barrier is required to extract charge into the multiplication region efficiently, and the extremely high density of field lines in the LEM causes the drifting electrons to avalanche, multiplying the charge to be read out by the anode strips.

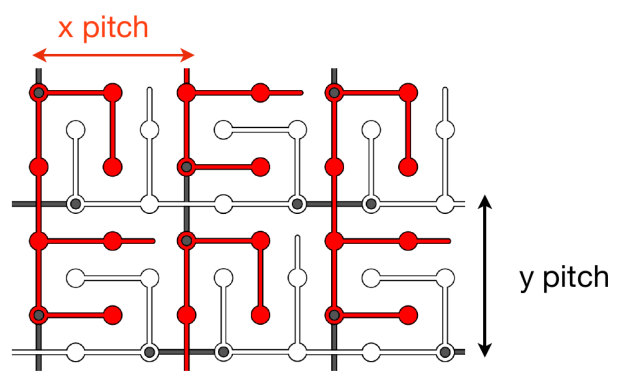

Fig. 2.19 Diagram of low capacitance pixel-based charge readout anode [132]. 


\subsection{Summary}

LAr is an excellent detection medium for energetic particles because of its ability to absorb energy and convert it into a detectable charge or light signal. It is a good calorimeter due to its excellent energy resolution, and its high electron mobility allows drifting of charge over large distances. There are several significant drawbacks to using LAr as a detection medium. These include, for example, the attenuation of charge by low concentrations of electronegative impurities, the presence of unstable ${ }^{39} \mathrm{Ar}$ decays, and the inability to achieve significant electron multiplication even in high electric fields. Two-phase argon TPC technology exists to overcome this last limitation.

Within the past four decades, aspects of building, operating, and analysing the data from LAr TPCs have been extensively studied in numerous prototypes and several full physics experiments. The LAr TPC concept has been chosen by the DUNE collaboration as the detector technology most suitable for performing the most sensitive neutrino oscillation measurements in history. The DUNE 35-ton LAr TPC discussed in the next chapter is an example of a prototype used to test some of the physics and engineering challenges of DUNE.

LAr TPCs will be important in particle physics for many decades to come. Still, there is much research and development needed to better understand particle interactions in LAr and the behaviour of free charges in a LAr TPC. 


\section{Chapter 3}

\section{The DUNE 35-ton Prototype Detector}

The Deep Underground Neutrino Experiment (DUNE) consists primarily of a massive liquid argon (LAr) time projection chamber (TPC) being developed and constructed in the next two decades. The 35-ton Prototype experiment was conducted to test and solve some of the initial challenges associated with scaling up the size of the DUNE LAr TPC. This chapter describes the 35-ton cryostat, detector, and experiment in detail, in preparation for the analysis of 35-ton data in Chapter 6. Also, a study by the author is presented in which the coordinate offsets between the lab frame and muon detector components were determined.

\subsection{The 35-ton Experiment}

The DUNE (née Long Baseline Neutrino Experiment) 35-ton Prototype experiment was separated into two phases, each with particular goals. The primary goal of Phase I was to test the suitability of the liquefied natural gas membrane cryostat for a high purity LAr experiment (see Figure 3.1), including thermal performance, leak tightness, and ability to achieve and maintain very high purity without evacuation [133]. Successful results make possible the construction of massive cryostats for the next generation of LAr TPC experiments.

Phase II of the experiment consisted of building and testing a TPC in the 35-ton cryostat (see Figure 3.2). The TPC was built with DUNE-specific design elements such as two-sided wrapped wire anode plane assemblies (APA), multiple drift directions, multiple drift volumes with more than one APA, and cold preamplification and digitisation electronics. It is important to note that the decision to install a TPC inside the cryostat was made after the final engineering design of the cryostat was finalised, which has significant implications for the performance of the detector as a whole (see Section 3.4.1). The goals of the Phase II experiment included (but were not limited to) studying the impact of gaps between the APAs, studying the electronics noise contribution from potential sources like pumps and acoustics, 


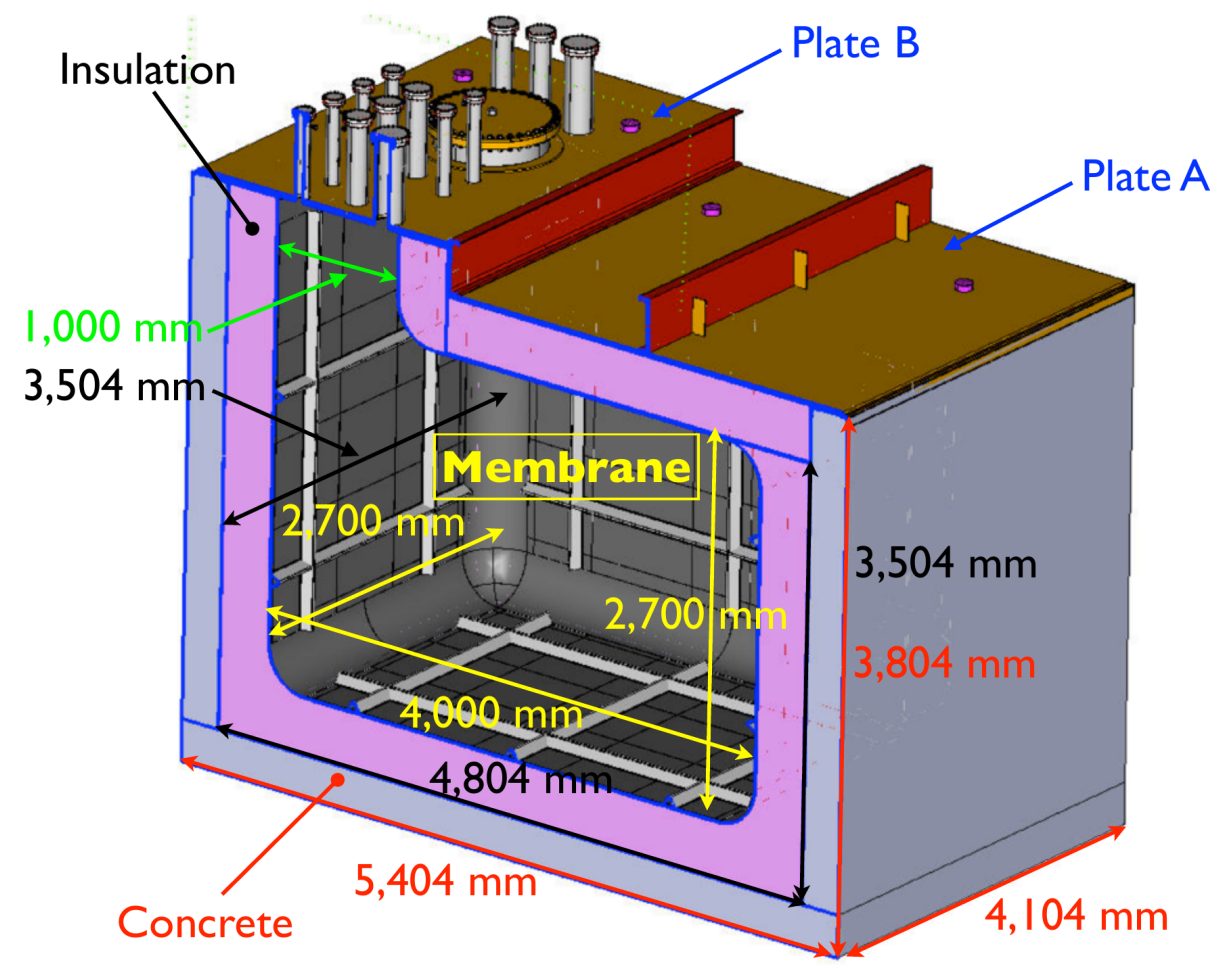

Fig. 3.1 Cutaway illustration of the 35-ton cryostat dimensions and construction details [134].

and studying the positive ion space charge distortion on muon tracks and its dependency on drift field and fluid flow.

\subsection{5-ton Detector Description}

The components of the 35-ton detector are described here and in more detail in [135]. For Phase I, only the cryostat, purification, and purity monitoring hardware were installed and used. For Phase II, the TPC, muon detectors, photon detectors (PD), and associated data acquisition (DAQ) hardware were added.

\subsubsection{Cryostat}

The 35-ton cryostat (Figure 3.1) was built using membrane cryostat technology originally developed by the liquefied natural gas industry. These cryostats are commonly built to keep large quantities (>250,000 $\mathrm{m}^{3}$ [134]) of predominantly liquefied $\mathrm{CH}_{4}$ below its $-162^{\circ} \mathrm{C}$ boiling point for transportation and storage, so the adaptation of the technology for storing DUNE-scale quantities $\left(\approx 7000 \mathrm{~m}^{3}\right)$ of $\operatorname{LAr}\left(-186^{\circ} \mathrm{C}\right)$ should be minimal. The primary motivation for using this technology is its cost-effectiveness in comparison to the traditional 


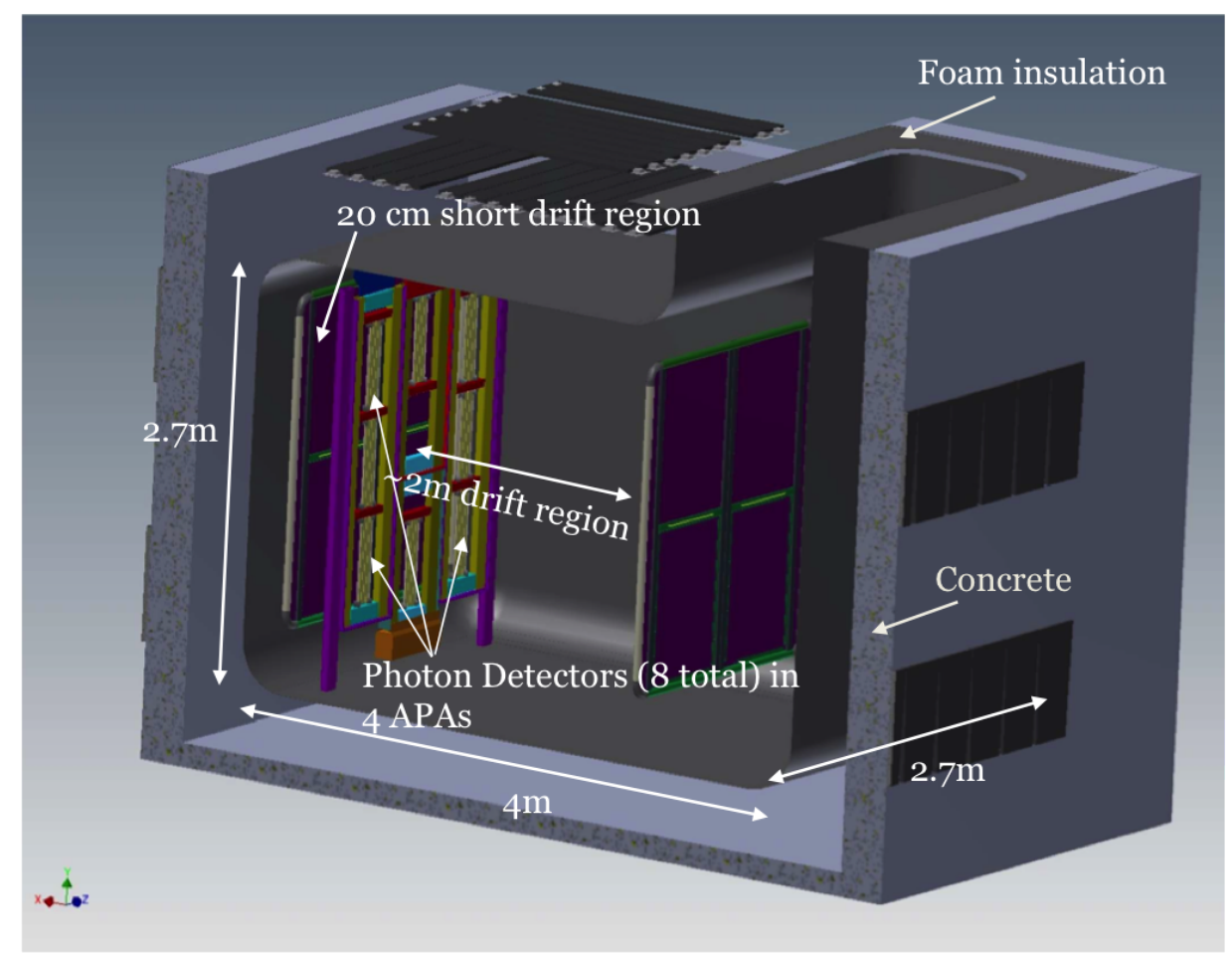

Fig. 3.2 Diagram of 35-ton TPC inside cryostat.

construction techniques for building scientific cryostats. The DUNE 35-ton cryostat has a total volume of about $29 \mathrm{~m}^{3}$ and could contain a total mass of 38.6 metric tons of LAr.

\subsubsection{LAr Purification and Monitoring}

The purification, liquification, and recirculation support hardware for the 35-ton were provided by the nearby Liquid Argon Purity Demonstrator (LAPD) [136]. The purification of LAr from electronegative contaminants has been extensively studied (for example, [137, 138, 139, $140,141]$ ) with the greatest reported electron lifetime ( $>20 \mathrm{~ms}$ ) achieved by using a copper and molecular sieve regenerable filter [142]. This purification scheme is used by LAPD and, hence, the 35-ton. Such a filter contains copper pellets which capture oxygen molecules by the adsorption reaction

$$
2 \mathrm{Cu}+\mathrm{O}_{2} \rightarrow 2 \mathrm{CuO}
$$

and molecular sieve to absorb water molecules in the microporous structure. The filter is regenerable through careful heating. For the copper, flowing hydrogen at an elevated temperature across the filter causes the reverse chemical process

$$
\mathrm{CuO}+\mathrm{H}_{2} \rightarrow \mathrm{Cu}+\mathrm{H}_{2} \mathrm{O} \text {. }
$$


For the molecular sieve, the elevated temperature simply evaporates the trapped water. By continuous recirculation of the LAr volume through the regenerable filter, extremely high argon purities are achievable without evacuation.

The primary measurement of argon purity in the 35-ton used the ICARUS style purity monitor (PrM) design [81], which is a gridded ionisation chamber, see Figure 3.3. A photo of one of the 35-ton PrMs is shown in Figure 3.4. In these devices, electrons are created at the cathode and allowed to drift through the test volume of LAr by the application of a uniform electric field. As a result of electronegative impurities, not all of the charge created at the anode reaches the cathode. So, the total charge measured (integrated current) on the anode and cathode are compared, thus revealing the electron lifetime in the sample:

$$
\frac{Q_{A}}{Q_{C}}=e^{-t / \tau}
$$

where $\frac{Q_{A}}{Q_{C}}$ is the ratio of measured charges, $t$ is the drift time, and $\tau$ is the measured lifetime. The lifetime is related to the impurity concentration, as described in Section 2.2.3.

In the 35-ton Prototype, five PrMs were installed to measure the argon purity at different locations within the cryogenic system [143]. One was located in the argon stream immediately following purification, before being pumped back into the cryostat. The other four were placed at different locations and elevations inside the cryostat to measure possible purity gradients.

\subsubsection{TPC}

Motivated by the DUNE far detector design requirements, the 35-ton TPC was segmented with multiple APAs and multiple drift directions. The 35-ton design consisted of two detector volumes, with a long drift $(2.2 \mathrm{~m})$ and a short drift $(0.3 \mathrm{~m})$, with outer cathodes and central anode, see Figure 3.2.

The TPC was segmented into eight distinct drift regions, front and back for each of the four APA frames. This geometry provided distinct gaps between sensitive readout regions between APAs, two vertical gaps between side-by-side APAs, and one horizontal gap between stacked APAs. All APAs were $0.5 \mathrm{~m}$ wide; the two tall ones were $2 \mathrm{~m}$ tall, the centre upper APA was $1.2 \mathrm{~m}$ tall, and the centre lower APA was $0.9 \mathrm{~m}$ tall. The electronics for reading out the wires were mounted on one end of each APA. In comparison to the DUNE far detector APA design, the 35-ton APAs had a slightly narrower aspect ratio, but similar in that there were still horizontal and vertical gaps between sensitive regions. 


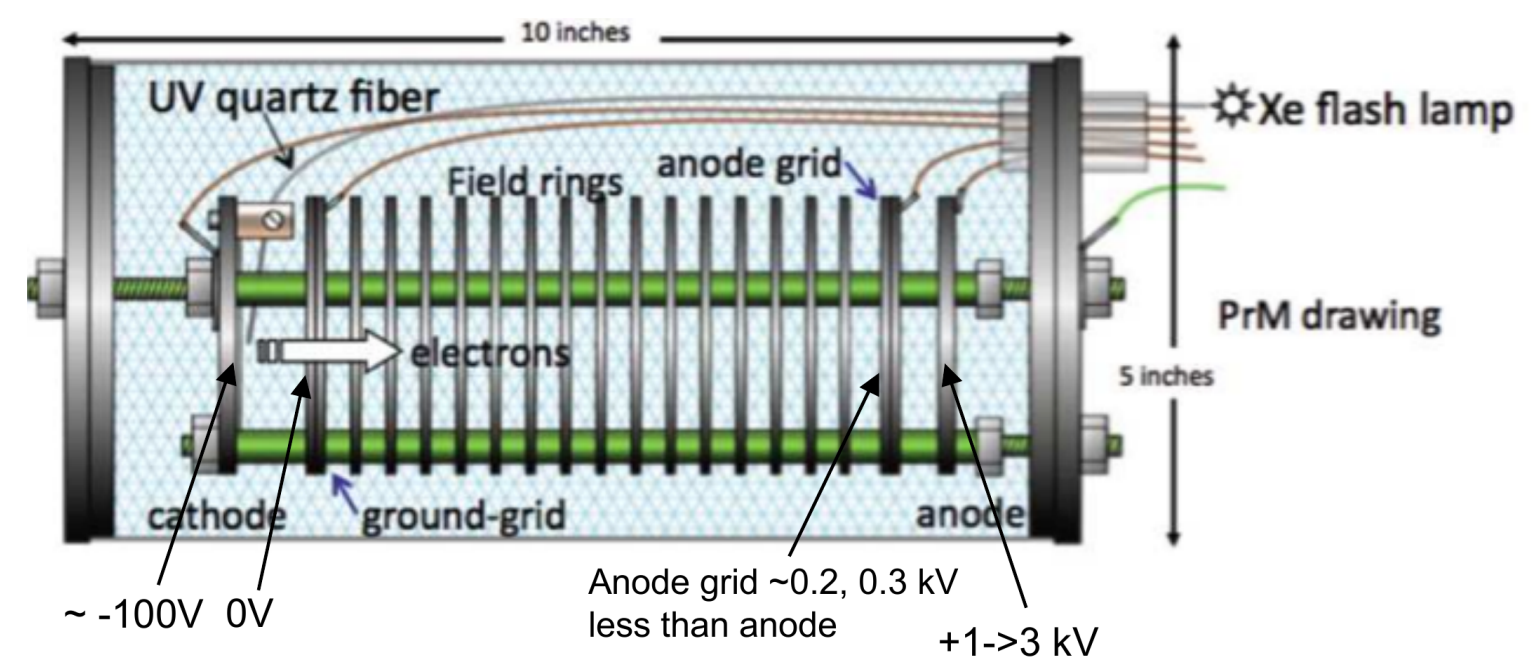

Fig. 3.3 Diagram of an ICARUS style purity monitor.

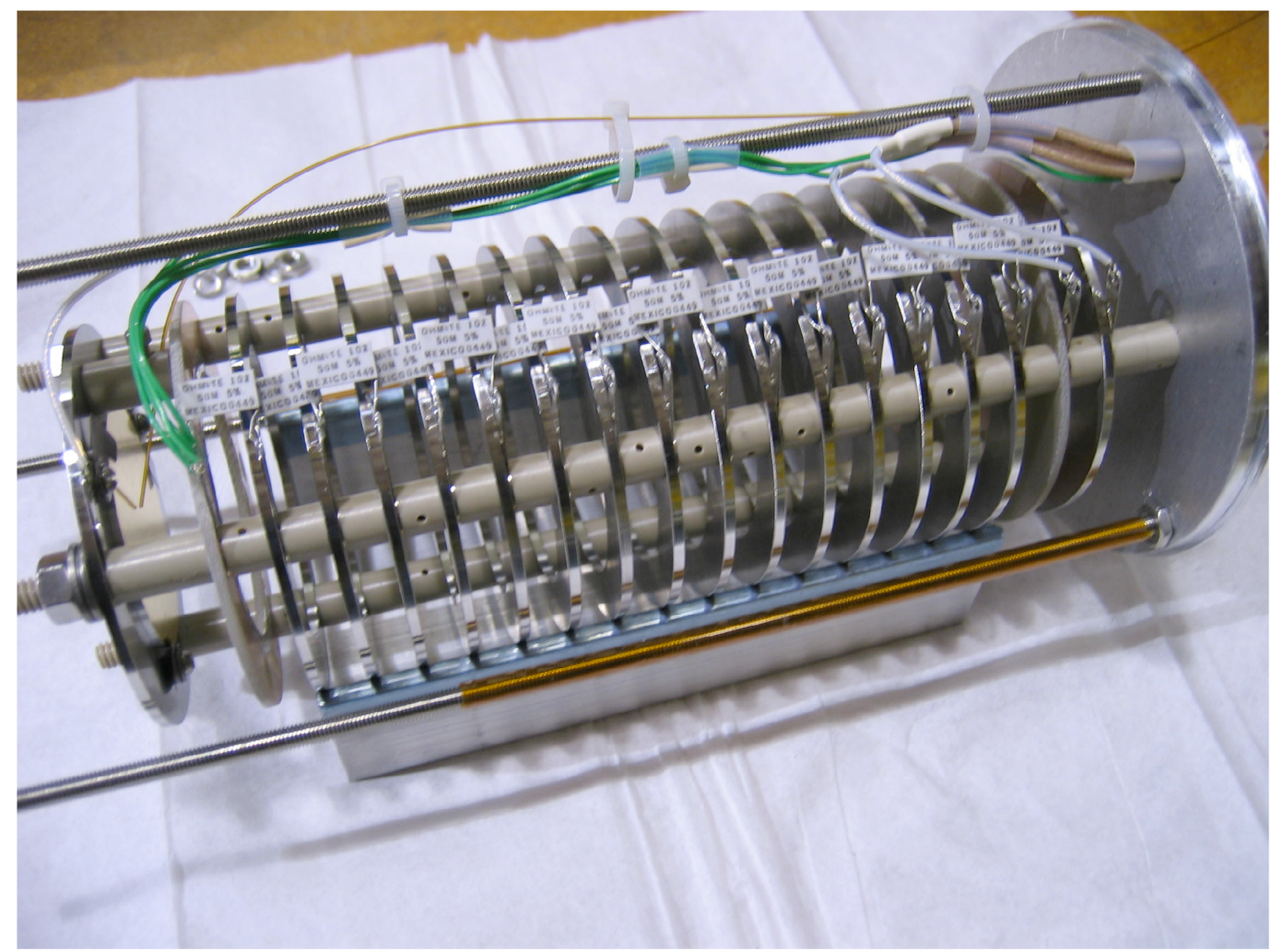

Fig. 3.4 Photo of a 35-ton purity monitor. 
The detector coordinate system was defined as in Figure 3.5 such that the origin is located at the same $y$ position as the centre of the horizontal gap, at the $z$ location of the very first collection plane wire on APA 0 , and at the $x$ location of the first induction plane wire on the long drift side of APA 0 . The electric fields were aligned along the $x$ axis, perpendicular to the APA planes. Detector hall "South" pointed roughly along the $+x$ direction, while "West" pointed roughly along the $+z$ direction.

The wire planes on each APA were designed such that the collection wires lie parallel to the $y$ axis, and the induction plane wires at $+45^{\circ}$ and $-45^{\circ}$ to the collection wires (Figure 3.6). The collection plane wires, or " $Z$ " wires, were separated by $4.5 \mathrm{~mm}$ in the $y z$ plane, and the two induction planes, " $U$ " and " $V$ ", had wire-to-wire spacings of $5 \mathrm{~mm}$ each. A fourth uninstrumented plane of wires, the "grid plane", was installed on each APA to shield the first induction plane from current induction due to charge drifting from very long distances. The separation distance between consecutive wire planes was $4.8 \mathrm{~mm}$. To reduce the number of readout channels, the induction plane wires wrapped from one side of the APA to the other, sometimes (in the case of the tall APA frames) more than once. The double wrapping of some wires caused ambiguous readout because there was more than one point where one wire from each plane intersects the same $y, z$ location. While this was the case for the 35-ton Prototype, the recently updated DUNE far detector design uses $\pm 35.7^{\circ}$ induction plane wrapping angles to prevent these ambiguities from occurring.

On each APA, there were 144 of each $U$ and $V$ induction plane wires, each reading out signals from both sides of the APA. Each side of each APA had $112 \mathrm{Z}$ collection wires, each reading only the signals from that side of the APA. Thus, the entire detector had 2048 total instrumented wires: 896 collection plane wires and 1152 induction plane wires.

The electric fields inside the detector were designed such that electrons in the drift regions experience a uniform field over the entire drift distance. Each plane of APA wires is biased such that the electric field lines never terminate on any wire except for the collection plane. This is accomplished by choosing the electric fields in these interplane regions such that Equation 2.1 is satisfied.

\subsubsection{TPC Data Acquisition}

The induced currents on each of the $U, V$, and $Z$ wires were amplified and digitised by front-end electronics mounted on the APA frame, submerged in the LAr. The preamplifiers had selectable shaping time and gain settings. For the majority of the 35-ton run (for the entirety of the data sample presented in Chapter 6), a shaping time of $3 \mu$ s and gain of $14 \mathrm{mV} / \mathrm{fC}$ was used. Signals were digitised at a rate of $2 \mathrm{MHz}$, each $500 \mathrm{~ns}$ analogue-to-digital (ADC) sample time is denoted as a "tick". A voltage offset ("pedestal") was added to each 


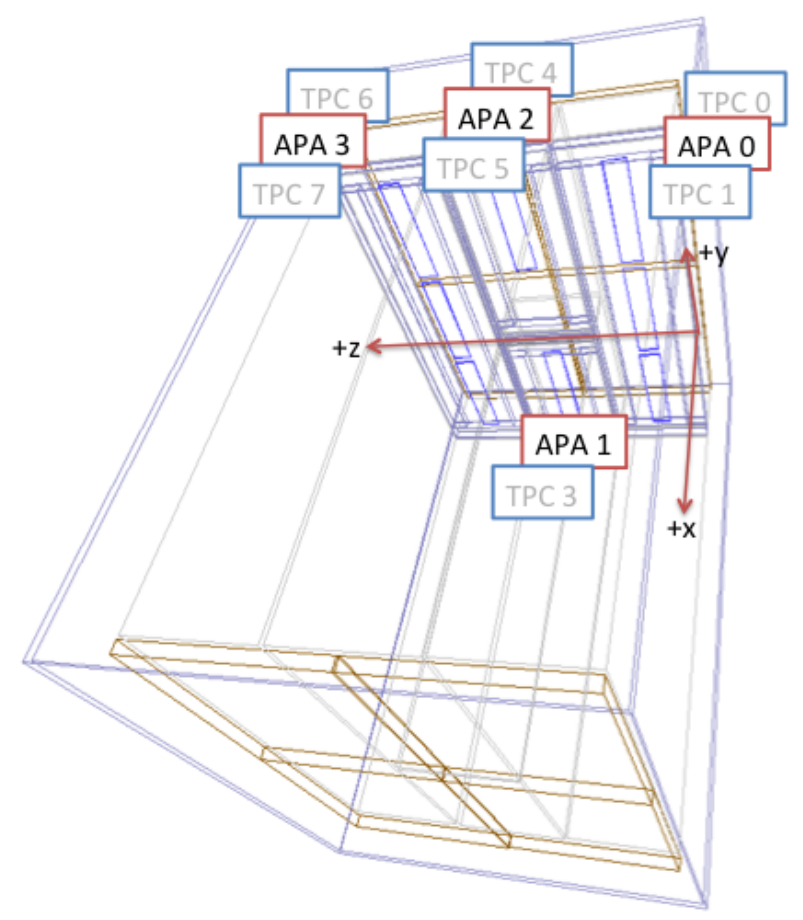

Fig. 3.5 TPC coordinate and numbering system.

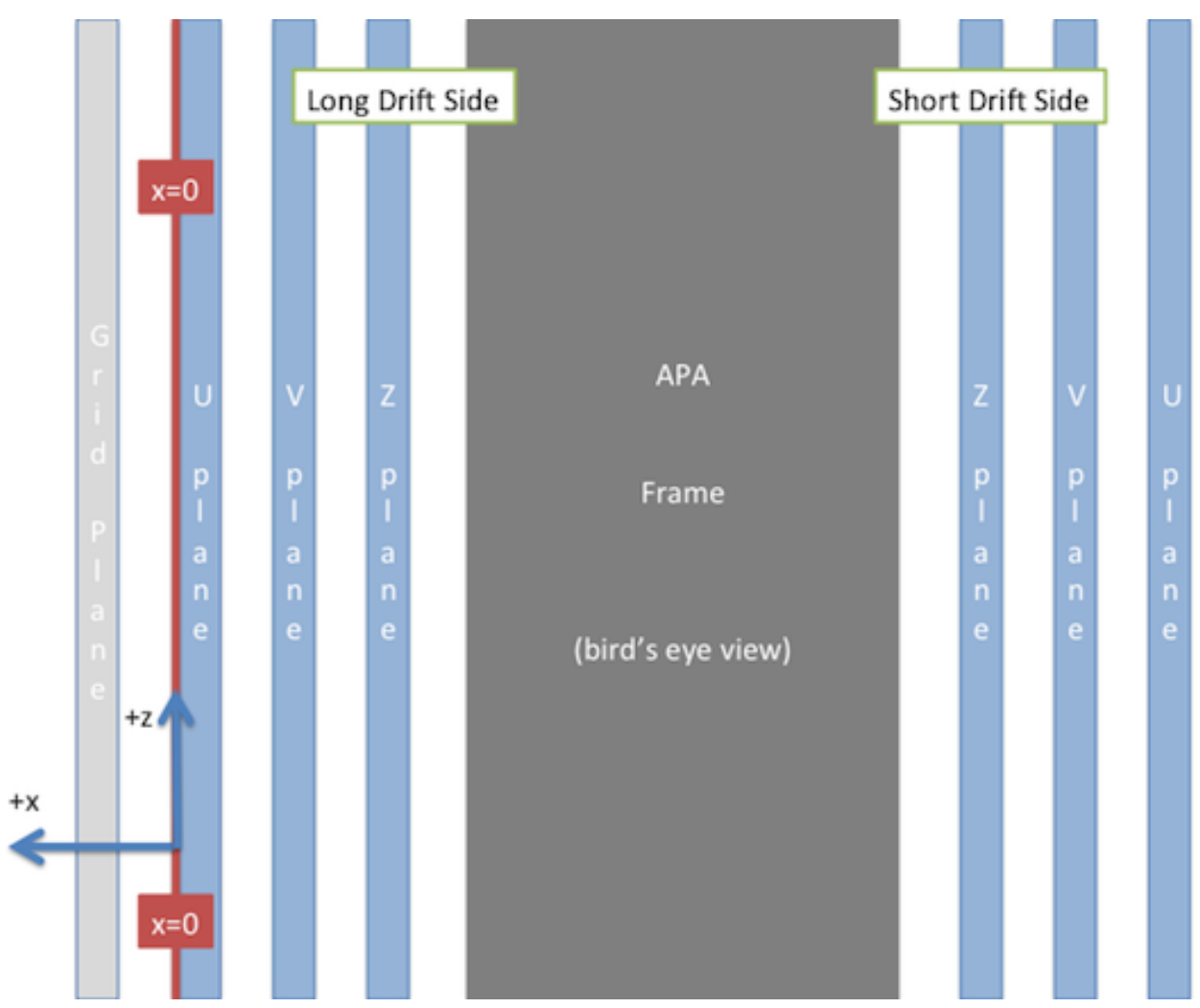

Fig. 3.6 Diagram of wire planes on APAs in the 35-ton Prototype TPC. 
signal to make digitisation of bipolar signals more straightforward. Digitised signals were sent out of the cryostat to so-called Reconfigurable Computing Elements which handled the triggering, buffering, and formatting of the data for storage. The electronics and the offline format (i.e. analysable by LArSoft) were interfaced using the artdaq framework [144], which stored the data in ROOT [145] format.

Each triggered readout from the TPC, called an "event", consisted of 15000 ticks, or $7.5 \mathrm{~ms}$. The trigger signal came from the muon detector system (described in Section 3.2.5) which signalled the TPC readout to build an event. Each event consisted of 4000-5000 buffered ticks before the trigger and the remainder of the 15000 total ticks following the trigger to give sufficient time for the triggering particle signal to be drifted to the anode and read out.

\subsubsection{Muon Detectors}

Because the 35-ton Prototype detector was not in any test beam, it was instrumented with 92 muon scintillation counter detectors for event triggering on cosmic particles. Figure 3.7 shows the locations and numbering scheme of all installed counters. 44 of the counters were designed to tag throughgoing muons at shallow angles, and the remaining placed above the cryostat to create a telescope to tag downward going muons. The triggering scheme was designed to tag specific coincidences of muon counters:

- Trigger ID 110 - Telescope: at least one event in each of the four planes of muon counters above the cryostat, 44-92.

- Trigger ID 111 - East-Lower / West-Upper: at least one event in counters 6-15, and at least one event in counters 28-37.

- Trigger ID 112 - North-Upper / South-Lower: at least one event in counters 22-27, and at least one event in counters $0-5$.

- Trigger ID 113 - South-Upper / North-Lower: at least one event in counters 38-43, and at least one event in counters 16-21.

The time threshold for recording a coincidence between two measured counter signals was $483.75 \mathrm{~ns}$, which is 40 clock cycles of the timing unit.

The telescope muon counters were not used in any analysis due to the high trigger rate and because vertical-going muons would pass nearly parallel to the collection plane wires. This event topology was very difficult to reconstruct because, in each event, nearly all of the event charge would be deposited on just a few collection plane wires, giving very large and possibly long charge deposits. Therefore, the horizontal triggering muon counters were used. 

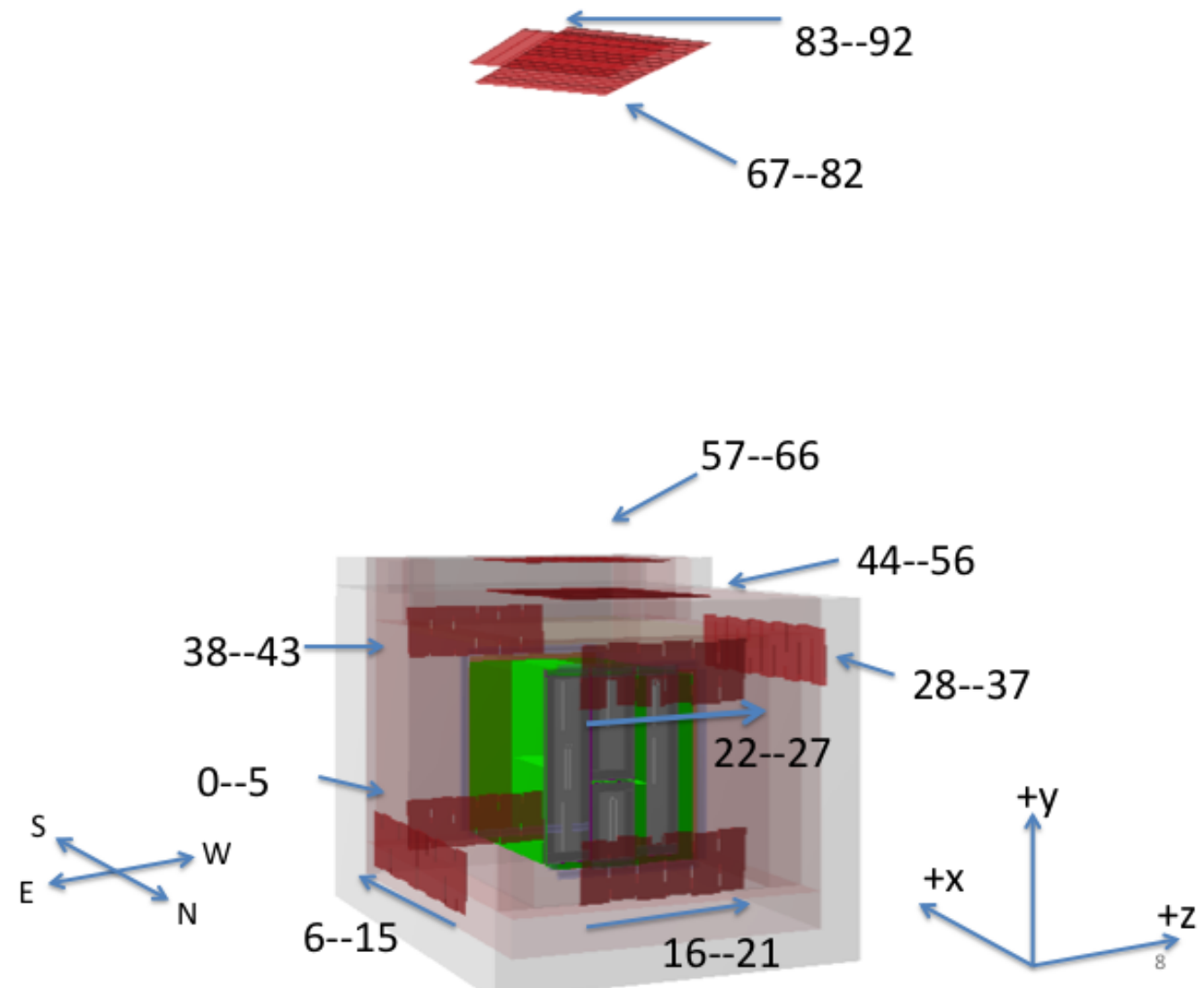

Fig. 3.7 Muon counters location and numbering with respect to the 35-ton cryostat and TPC, with the detector coordinate system. 
Each muon counter on the vertical cryostat walls was trapezoidal, $63 \mathrm{~cm}$ tall, $27.2 \mathrm{~cm}$ wide at one end, and $32.5 \mathrm{~cm}$ wide at the other end. The sensitive area of each counter, $O\left(10^{3}\right) \mathrm{cm}^{2}$, was large with respect to the position resolution of the TPC, $O(1) \mathrm{cm}^{2}$, making accurate positioning of crossing muon tracks ineffective from the muon counter system alone.

Particles invoking any of triggers 111,112 , or 113 were nearly always guaranteed to traverse the active region of the TPC. Few exceptions include counter pairs $6 \& 28,6 \& 29$, $7 \& 28,37 \& 15,0 \& 27,38 \& 21,5 \& 22$, and $43 \& 16$, where some fraction of triggering events would pass through inactive regions (e.g. outside of the field cage) because of the high granularity of space covered by each muon counter. East-West triggers (ID 111) were used for most analyses because triggered events nearly always passed through the full width of the TPC, exposing each collection plane wire to the minimal possible track length, which gave the least probability for the ADCs to be saturated.

\subsubsection{Photon Detectors}

A system of wavelength shifting light guides instrumented with silicon photomultipliers (SiPM) was installed within the APA frames to measure the primary scintillation light from particle interactions in the LAr [146]. Eight separate PDs were installed in the 35-ton TPC, shown in Figure 3.8. Each SiPM was read out by a designated SiPM Signal Processor, which read the analogue waveforms, performed preamplification, and digitised the signals for readout.

Part of the goal for the 35-ton PD system was to test possible detector geometries for vacuum ultraviolet (VUV) photon collection for use in future DUNE prototypes and experiments. Therefore, PDs 1, 3, and 7 consisted of acrylic rectangular light guide bars, coated in a Tetraphenyl Butadiene (TPB) solution to shift the wavelength of light from the $127 \mathrm{~nm}$ emitted by LAr scintillation to the visible range, to which the SiPMs mounted on the ends were sensitive. PD 2 used an acrylic plank, also coated with TPB, with an embedded bundle of wavelength shifting fibres. PD 5 used the same waveguide four-bar design as PDs 1,3 , and 7, but with a different method of TPB coating. Finally, PDs 0,4 , and 6 consisted of bundles of wavelength shifting fibres placed around a TPB-coated radiator. The performance of the PD system is described in [146].

\subsection{Phase I}

Construction of the cryostat was completed in 2012, the cryogenic support system completed in 2013, and the full Phase I run completed in 2014. Rather than being evacuated, the cryostat 


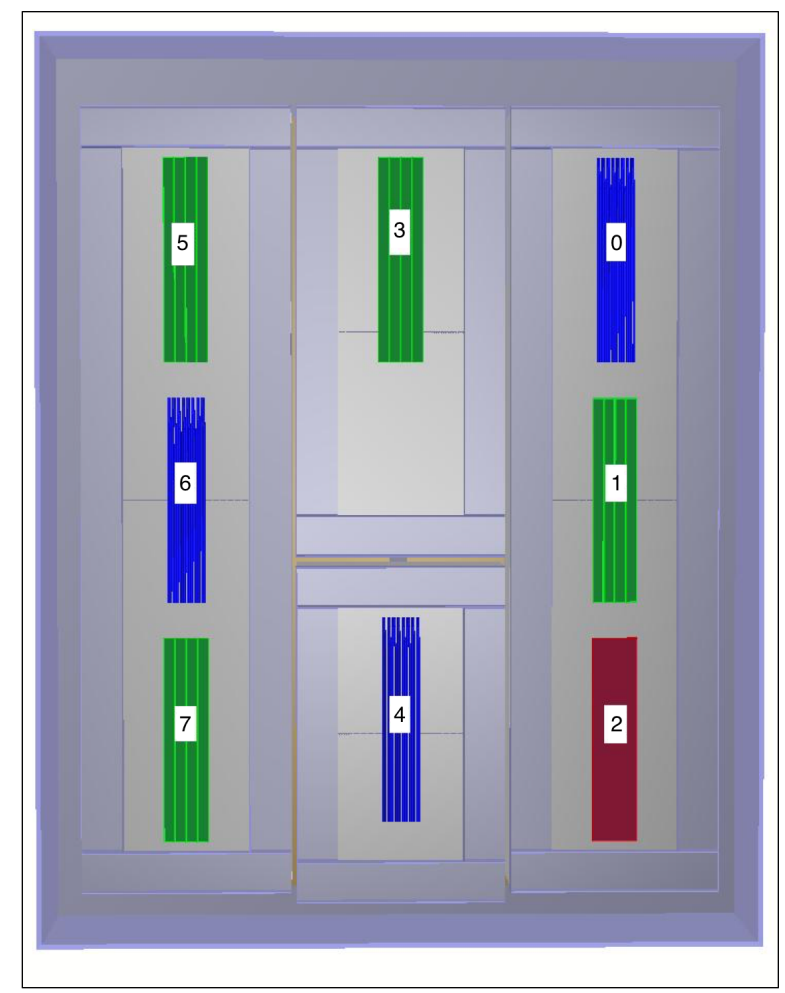

Fig. 3.8 Locations and numbering scheme for the PDs in the 35-ton TPC.

was purged with argon gas before filling with LAr. Cooldown occurred at less than $10 \mathrm{~K}$ per hour by a vapour-phase system whereby cold and room temperature argon gas were sprayed simultaneously at varying rates to slowly and evenly cool all components to $-186^{\circ} \mathrm{C}$. Once at the appropriate temperature, the cryostat was filled with pure LAr which was continuously recirculated through the purifiers to remove any impurities introduced by the cryostat or detector components.

For most of the Phase I run, the measured electron lifetimes are shown in Figure 3.9. Following the initial turn-on of the recirculation pump, and another instance where the LAr was topped off, within 6 LAr volume changes the lifetime was consistently measured above $1 \mathrm{~ms}$. The required minimum purity of $1.4 \mathrm{~ms}$ lifetime was achieved after 14 volume changes of the recirculating LAr. The LAr volume and minimum purity were maintained for approximately two months when Phase I was completed, indicating the successful accomplishment of the phase goals. 


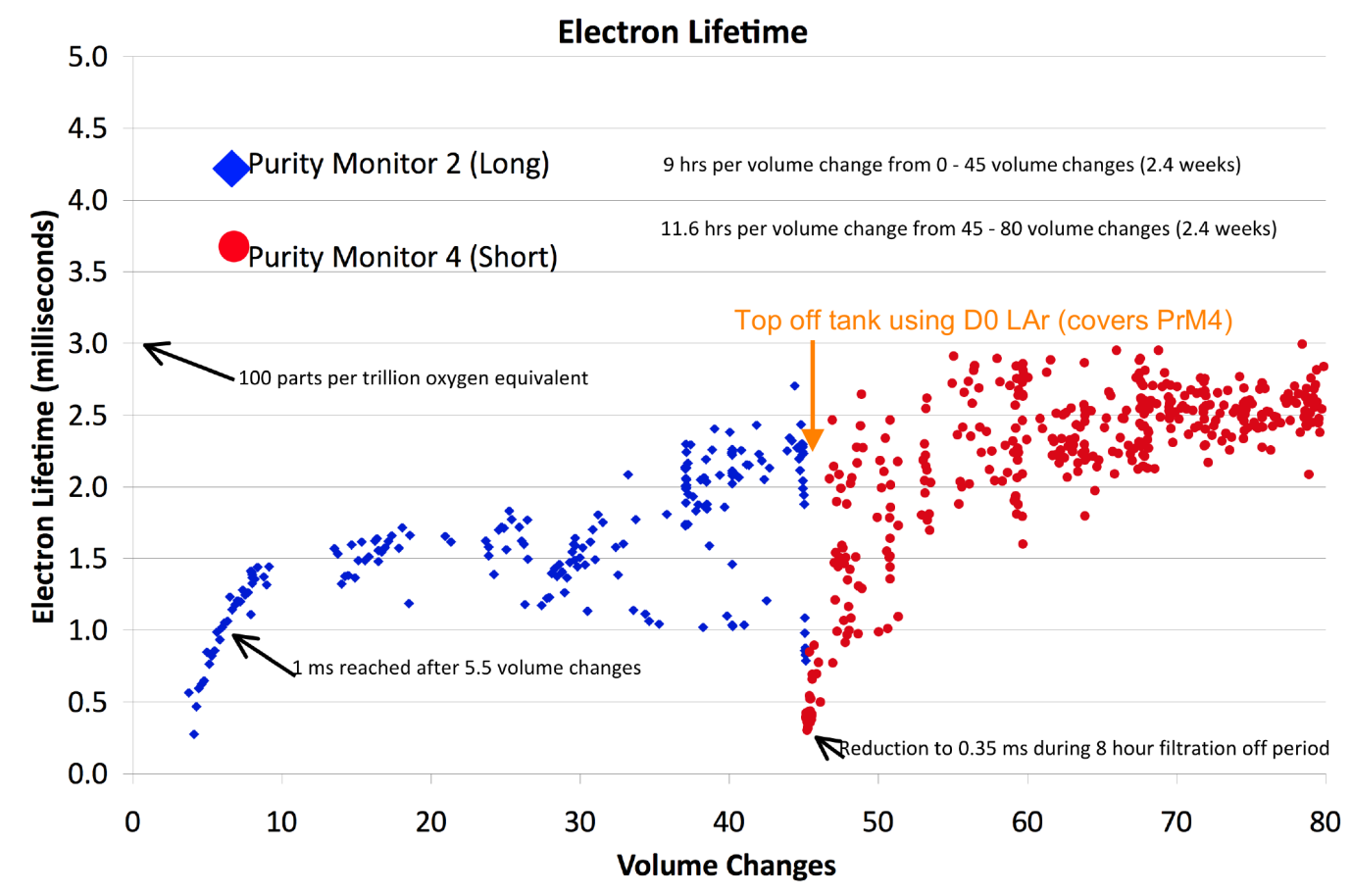

Fig. 3.9 35-ton Phase I electron lifetime as measured by the PrMs.

\subsection{Phase II}

The 35-ton Phase II run began in January 2016, following the installation of the TPC and other detector components, and ended 19 March 2016. During the run, the drift field was $250 \mathrm{~V} / \mathrm{cm}$, half of the nominal field for the full DUNE far detector design. The electron lifetime of the argon, as measured by the PrMs, was around $3.0 \mathrm{~ms}$ for the duration of the data taking run. The required electron lifetime was achieved in a membrane cryostat with a fully instrumented detector, an important milestone for the next generation LAr TPC experiments. The 35-ton also showed stable high voltage operation with no indications of field non-uniformities in particle tracks. Sufficient data were recorded to accomplish basic studies such as $[135,146]$

- photon detector / TPC event time resolution

- argon electron lifetime (see Chapter 6 of this work)

- signal-to-noise ratio

- analysis of charge loss in APA gaps and between wire planes of the same APA

- study of event timing based on charge diffusion

- analysis of timing differences between APAs 
in addition to fine-tuning the LAr TPC simulation and analysis software to be used in subsequent experiments.

There were several operational, construction, and electronics issues which interfered with the data taking, including broken wires, cold failure of some front-end electronics, a long shutdown of all detector components, electronics degradation during the shutdown, and high electronic noise in the data. The run ended abruptly when a pipe failure caused unrecoverable air contamination of the full LAr supply, effectively reducing the electron lifetime to zero and saturating the purifiers with water ice.

\subsubsection{Noise}

The electronic noise was a significant factor of consideration and effort during the 35-ton Phase II run. Despite the expectation for the thermal noise to be low due to cryogenic cooling of the application specific integrated circuits (ASIC), the noise was higher than observed during testing. There were two distinct noise states for the detector. At worst, the noise RMS exceeded 200 ADC counts on every channel throughout the detector, called the high noise state (HNS). This was in contrast to the low noise state (LNS) where the RMS averaged about 30 ADC counts per channel. For context, the front end electronics processing of a minimally ionising particle (MIP) signal would typically create a pulse with amplitude of 100 ADC counts on a particular collection plane channel. Such a signal would be completely overshadowed by noise in the HNS, but still above a twice the noise RMS in the LNS. The detector would normally run in the LNS until, spontaneously, it would switch into the HNS until the front end boards were power cycled. The cause of the HNS was never determined, although it was observed that the detector would only enter this state while the drift field was applied and the detector submerged in LAr.

The analysable data were all taken while the detector was in the LNS. The FFT of an example event from this dataset is shown in Figure 3.10 where it is clear that there are distinct frequencies of noise affecting every channel, and wide variations in noise power between channels.

There are components of the noise which are correlated across all 128 channels on each front-end motherboard which has been attributed to a voltage regulator for the board's ASICs. In general, the main source of uncorrelated noise is attributed to the poor isolation between the detector and building grounds allowing a small leakage current. 


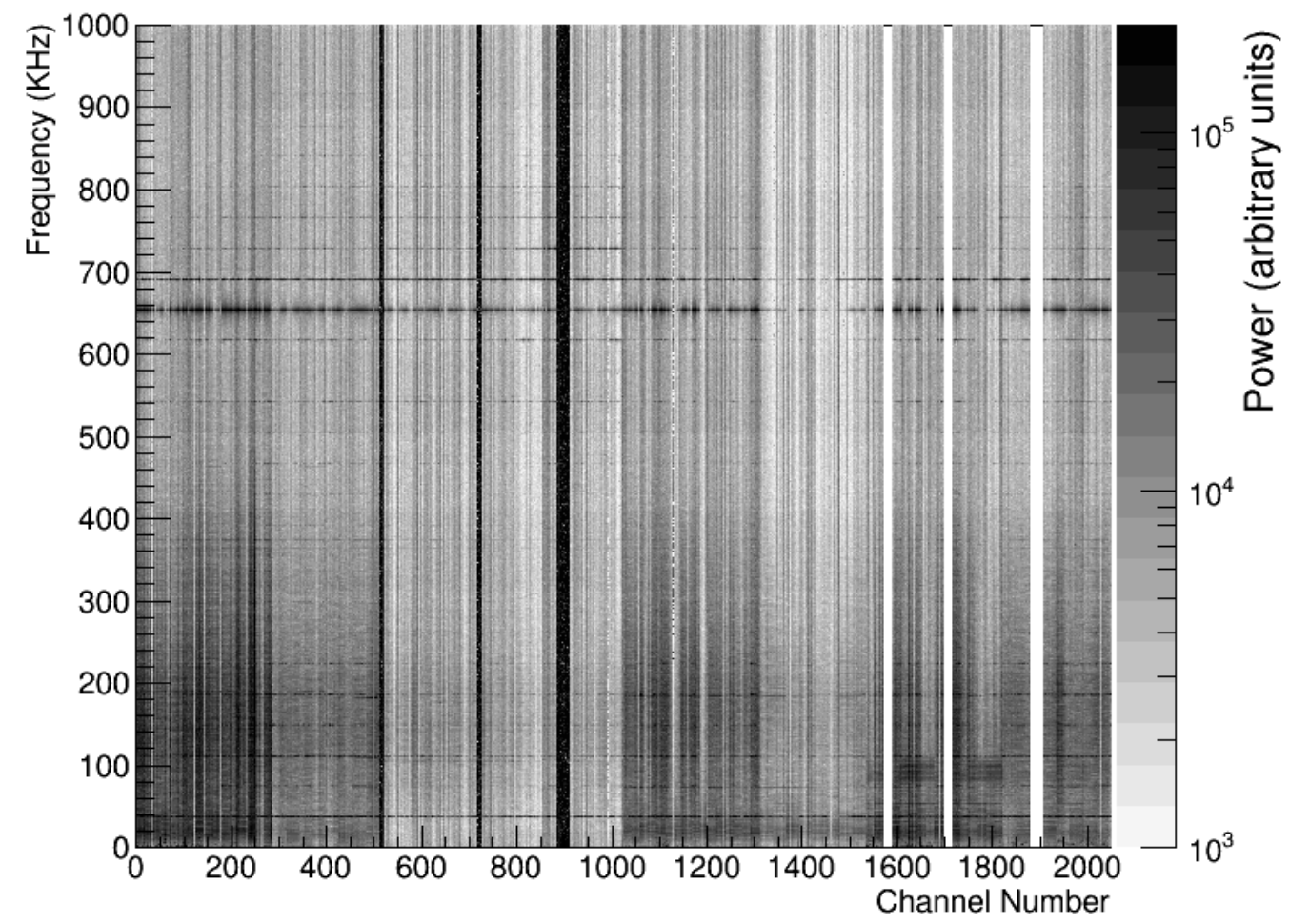

Fig. 3.10 The Fourier transform of the ADC values on all channels in the LNS.

\subsubsection{Stuck Bits}

Some of the data were corrupted at the bit level. Specifically, the low six bits of the 12-bit ADC would occasionally be incorrectly reported as $0 \times 0$ or $0 \times 3 F$, which caused the number represented by the upper six bits to be either higher by one or lower by one, respectively, than it would be if not stuck. The probability of this happening depends on many factors including temperature, channel, input current, and proximity of the true ADC value to the nearest $\mathrm{ADC}$ where the lowest six bits were $\mathrm{Ox} \boldsymbol{0}$. Once stuck, a channel may have remained stuck for several ticks at a time until the bits were completely cleared. The fractions of stuck ADCs in a waveform varied from $20 \%$ to $80 \%$, depending on the factors above.

\subsubsection{Purity}

Of the four PrMs in the 35-ton cryostat, the two long PrMs with drift time of $1.4 \mathrm{~ms}$ were more suited to measuring electron lifetimes greater than $1 \mathrm{~ms}$. Of the two, PrM 2 was thought to give the most representative electron lifetime of the whole cryostat for several reasons. First, it was mounted more than a metre from the surface of LAr once filled. This is significant because outgassed impurities are known to dissolve into the LAr, which, in 
principle, corresponds to an unrepresentative higher impurity concentration near the liquid surface. Second, given the locations of the LAr recirculation pump inlet and outlet ports, fluid flow simulations showed the best mixing of impurities and LAr temperatures were at the location of PrM 2.

The PrM 2 data (Figure 3.11) shows the measured electron lifetimes over the course of the Phase II run. Various external events are clear in the plot, such as initial purification which began on 14 February, the Fermilab power outage on 5 March, and the pipe break on 19 March when the lifetime suddenly dropped. For the data set used in the analysis, described in Section 6.1.3 and highlighted in red in Figure 3.11, the representative mean electron lifetime from the PrM system is $2.8 \pm 0.1$ (stat.) \pm 0.5 (syst.) ms. Results from LAPD [136], which tested the same devices and measurement technique, estimate a systematic uncertainty of about $5 \%$ on the $Q_{A} / Q_{C}$ measurement, which translates to about $10 \%$ error in reported electron lifetime.

\subsection{Calibrating Muon Counter and TPC Coordinates}

The muon counter system was used extensively in data analysis to apply fiducial cuts for searching the detector for relevant signals, and for event $T_{0}$ determination. For these cuts to effectively isolate the signals from the background, the relative coordinates of the muon counter system and the TPC must be understood. In particular, the location of the TPC in the lab frame was known less precisely than the location of the muon counters, because the TPC was not accessible for survey once the cryostat was closed.

Upon first look at the 35-ton data, discrepancies were observed between the reconstructed through-going muon track and the assumed region of acceptance bounded by the triggered muon counters. These manifested as offsets between the two coordinate systems. Because the analysis initially used only collection plane wires, this discrepancy was more difficult to observe independently of other possible systematics, such as timing offsets between the TPC and muon counters. Also, since the collection plane wires were parallel to the $y$ axis and spanned up to $2 \mathrm{~m}$ of distance, the $y$ coordinate shift between the two reference frames was indeterminable.

Possible discrepancies in coordinate systems were determined in two ways. First, by extrapolating reconstructed tracks from the Track Hit Backtracker (Section 5.3.4) in the TPC frame, toward the location of the muon counters on the wall where they were mounted, differences in position were observed. A grid search method was applied where the counter locations were shifted along the $x$ and $z$ directions, and the number of track intersections was counted. Within the grid search, the relative locations of counters within each grouping are 


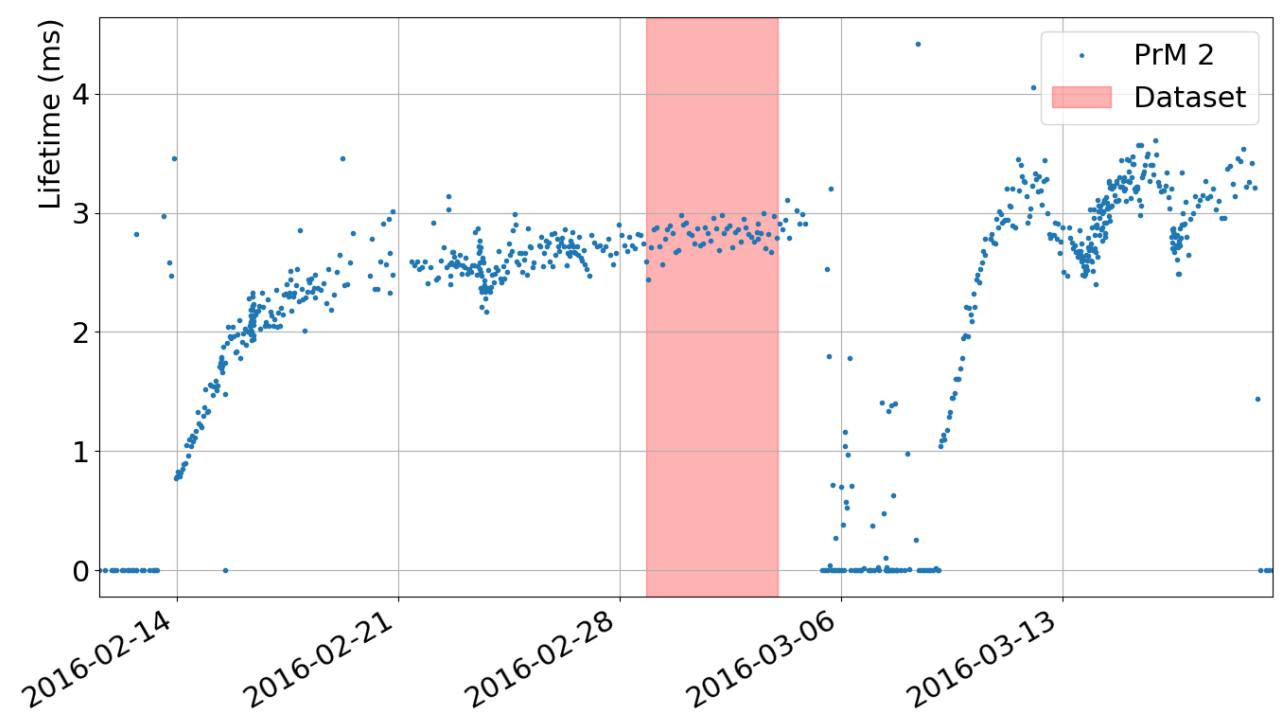

Fig. 3.11 Measured electron lifetime by PrM 2 over the course of the Phase II run. The shaded region represents the times when the analysed data were taken.

constrained, which is motivated by the physical layout of the linear grouping. Therefore, the offset is applied to the entire grouping of counters, rather than to each counter. The results of this are shown in Figure 3.12 for North and South counters separately. Both the $z$ and $x$ most probable offsets (the location with the highest number of track intersections) are within one standard deviation of zero.

Another method for determining the $z$ offset of the North-South muon counters is independent of both the $x$ offset and full event reconstruction. In this method, only events which are perpendicular to the APA are considered. First, every ADC in every channel waveform is standardised using the noise RMS for that channel. In the absence of signal, given random noise, every wire is equally likely to contain the largest standardised ADC value on every tick. But, in the presence of a long charge deposit from a perpendicular track, the wire which collects the charge is more likely to contain the largest ADC. Therefore, for every tick in an event, the location of the collection plane wire with the largest standardised ADC value is histogrammed for each counter pair. This premise is independent of hit reconstruction, which is known to fail for these types of particle tracks in the TPC. The results of this analysis are shown in Figure 3.13. The excess of events for the particular counter pair, fit to a Gaussian, illustrates the true location of the muon counter in the TPC frame. The $z$ coordinates of the North-South muon counters are found to be consistent between reference frames by comparing the Gaussian peaks with the measured locations of the counters in the 


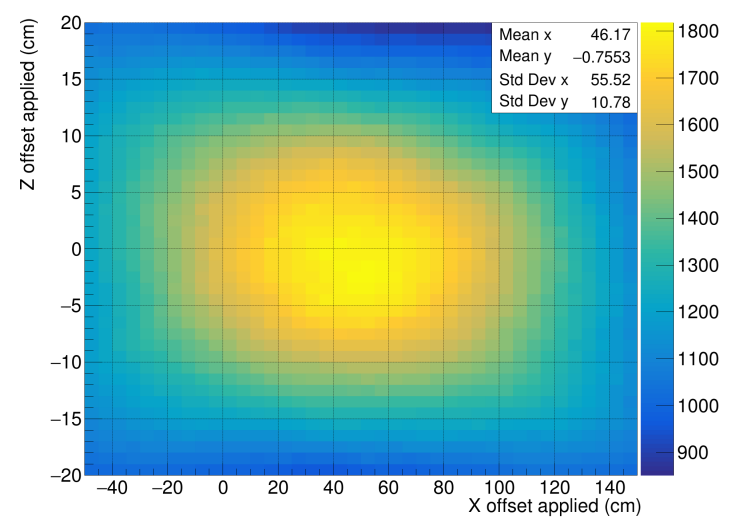

(a) South Counters (combined)

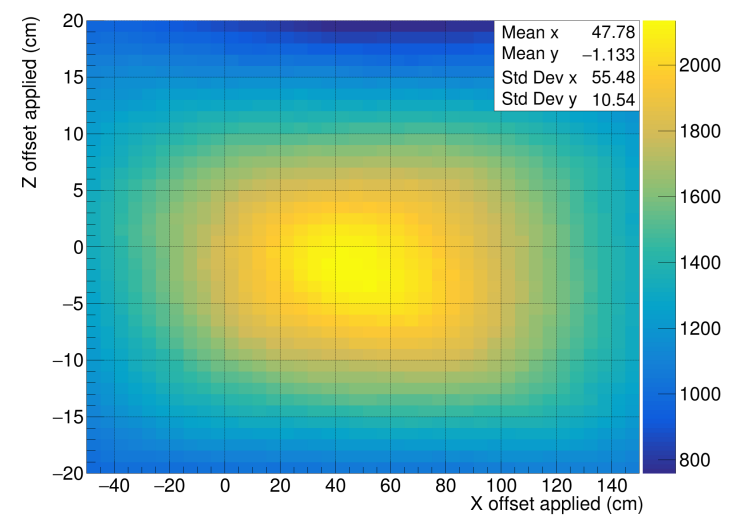

(b) North Counters (combined)

Fig. 3.12 Number of intersections of muon counter and reconstructed track for a given grid search offset applied in $x$ and $z$.

lab frame (dashed lines). For counter pairs $0 \& 27,38 \& 21,5 \& 22$, and $43 \& 16$, the scintillator paddles straddle the edge of the TPC, so triggered muons may not necessarily be read out by the detector, resulting in the narrow distributions shown.

Both methods of measuring the offset agree in that they predict the TPC $z$ coordinates to be consistent with the North-South muon counter $z$ locations. For the grid scan method, the North counter grouping is found to be offset from the TPC by $-1.1 \pm 10.5 \mathrm{~cm}$, and the South counter grouping by $-0.8 \pm 10.8 \mathrm{~cm}$. For the $x$-independent method, the measured $z$ coordinates reflect the offset to be consistent with zero (see Table 3.1). The two independent methods of measuring the coordinate offset both produced the same results, indicating with high likelihood that there is no significant difference between the TPC and North-South muon counter reference frame $z$ coordinates.

Table 3.1 Measured $z$ offset using $x$-independent method. *Note that counters $0,5,16,21$, $22,27,38$, and 43 straddle the edges of the TPC and are not reliable in measuring the $z$ offset.

\begin{tabular}{l|l} 
Counters & Measured Offset \\
\hline $0,21,27,38$ & $7.15 \mathrm{~cm}^{*}$ \\
\hline $1,20,26,39$ & $1.28 \pm 7.54 \mathrm{~cm}$ \\
\hline $2,19,25,40$ & $-1.03 \pm 9.24 \mathrm{~cm}$ \\
\hline $3,18,24,41$ & $0.18 \pm 11.29 \mathrm{~cm}$ \\
\hline $4,17,23,42$ & $-1.85 \pm 8.85 \mathrm{~cm}$ \\
\hline $5,16,22,43$ & $-11.29 \mathrm{~cm}^{*}$ \\
\hline
\end{tabular}




\subsection{Summary}

The 35-ton Prototype cryostat and TPC experiment were both valuable tests of the engineering and physics developments needed for scaling LAr TPC technology up for DUNE. The membrane cryostat was shown to keep high purity LAr for an extended period, even with various detector components installed. Additionally, lots of data were taken with the TPC, allowing ongoing studies of the physics, and also the validation of the engineering and systems designs. For example, ongoing investigations include tests of the PD system [146], intersystem timing validation, spatial offsets between APAs, and diffusion-based hit time determination [135].

Despite the issues uncovered during the Phase II run, such as high noise and broken pipes, the experiment was seen as a success in that the critical goals of the prototype were fulfilled. For example, studies have been completed which investigate the impact of gaps between the APAs and the electronics noise contribution from various sources [135]. One of the essential physics measurements is the determination of LAr purity using TPC data, which is described in Chapter 6. 

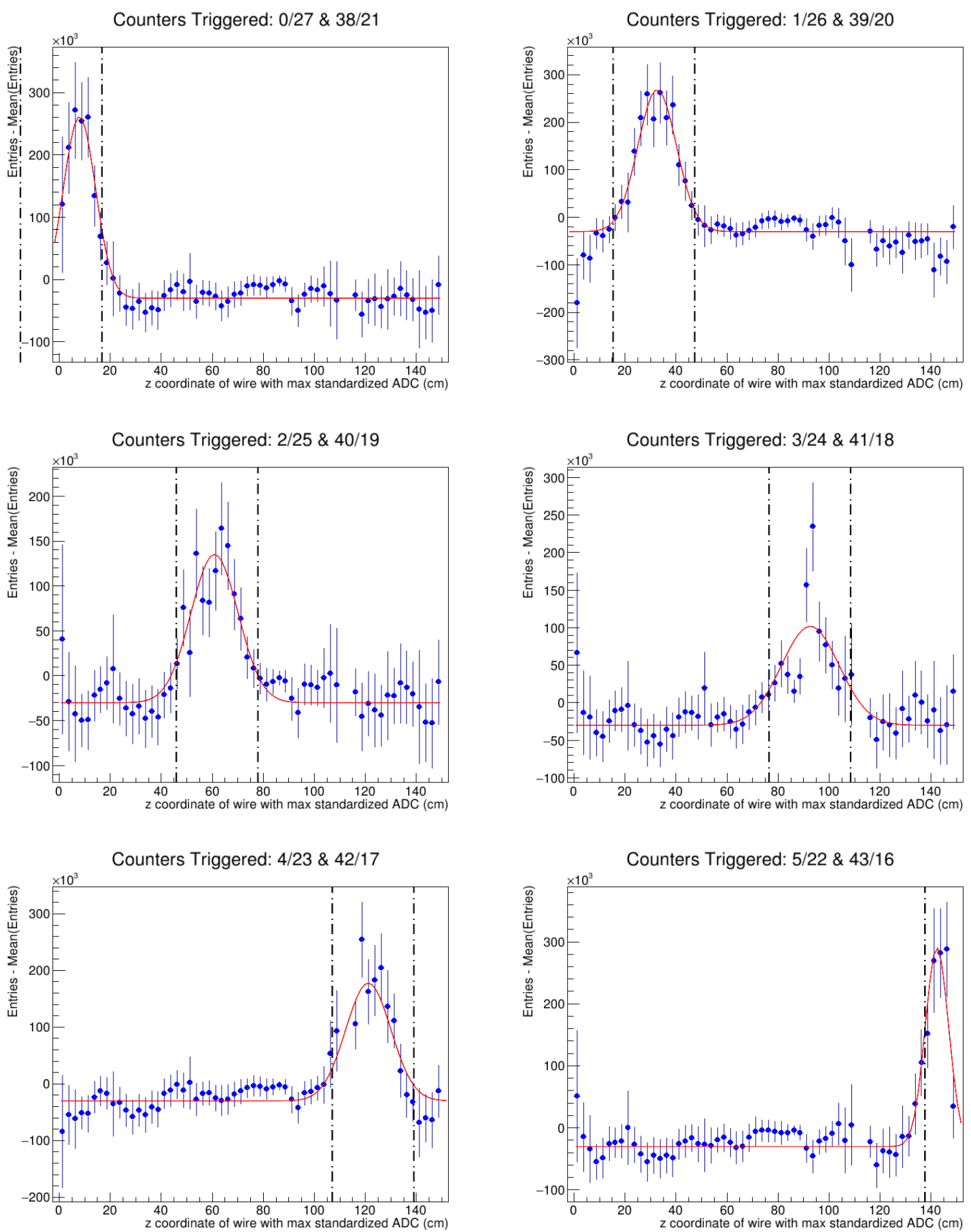

Fig. 3.13 Measured $z$ position of North-South muon counters by finding the wire with largest standardised ADC value for each TDC tick. Distributions are fit to Gaussian to obtain central value. Dashed lines indicate independently measured $z$ boundaries of the corresponding muon counter pairs. 



\section{Chapter 4}

\section{Cameras In The 35-ton Cryostat}

As liquid argon (LAr) time projection chambers (TPC) get larger and more complex, having a visual look at the inside of the cryostat after it is sealed is indispensable. The large forces applied on and energy transfers between the detector and cryogenic components during cool down, during application of high voltage (HV), and over time as the components settle and degrade may cause physical changes in the detector. These changes might be visually easily diagnosed if it were not for the fact that the cryostat is sealed. The obvious solution is to use cameras to monitor detector components in situ. However, this is a challenge because most modern electronics are known to fail at extremely low temperatures, and injecting additional heat into the cryostat is usually unacceptable.

This chapter recounts the development a system of cryogenic cameras installed in the Deep Underground Neutrino Experiment (DUNE) 35-ton Prototype cryostat. The full installed system is described in detail, as well as the collected results and studies of long-term camera behaviour in the cold. This system is the first such array of cameras developed for use in a large LAr cryostat, capable of functioning without extra heating. Much of this work is published by the author [147].

\subsection{Motivation}

There are several motives for using cameras in the cryostat of a LAr TPC experiment. First and foremost is the diagnosis of HV breakdown. In 1961, the electric breakdown field in LAr was measured to be $1.4 \mathrm{MV} / \mathrm{cm}$ [148]. However, recent research [149] has shown that for the current generation of LAr TPC experiments with long ( $\gtrsim 1 \mathrm{~m})$ drifts and very high argon purities, the breakdown field could be an order of magnitude lower, depending on anode-cathode material and geometry, and argon purity. Figure 4.1 shows the wide range of experimental measurements of the breakdown field [150]. For example, the 35-ton TPC 
has a drift distance of just over $2.2 \mathrm{~m}$, and a nominal design electric field of $500 \mathrm{~V} / \mathrm{cm}$, so the voltage supplied to the cathode via the feedthrough could be as high as $120 \mathrm{kV}$. If the HV supply line were near any grounded surface, or if a sharp edge created localised high fields more than $100 \mathrm{kV} / \mathrm{cm}$, a discharge could readily occur. These discharges can be especially dangerous because the sheer amount of energy released has the potential to cause irreversible damage to critical components of the detector. Because the research in this area is still ongoing, such breakdowns are not very well understood or predictable. Future experiments such as DUNE have even longer drifts, requiring much higher supply voltages, increasing the breakdown risk even further. Therefore, visual confirmation of the location of such breakdowns in the prototype detector can provide invaluable insights into the causes, motivating further improvements in the engineering of $\mathrm{HV}$ components.

Within the field, there is significant interest in the observation and visual diagnosis of electric discharge in TPCs. For example, SBND and protoDUNE are planning on installing HV monitoring cryogenic camera systems. A video of a discharge in a LAr TPC could assist in determining the location of the spark and could also differentiate between sparking and other situations in which the HV suddenly drops, which would be otherwise impossible to determine by simply monitoring the HV power supply. Such a system must be capable of running for long periods of time at LAr temperature without additional heating, and would not interfere with other critical detector components.

Another motive for using cameras in a LAr TPC experiment is the monitoring of various internal cryogenic support subsystems during cooldown, which, again, would be otherwise impossible to observe except through the warm-side process monitors. There is tremendous value in visual observation in the diagnosis of an engineering failure.

For all of the above reasons, a system of cryogenic-capable cameras was developed and installed in the 35-ton cryostat for the Phase II run. The details of the development, full system components, and operation are discussed in the following sections.

\subsection{Camera Selection}

The first and most important obstacle in developing a system of cryogenic cameras is to select a device which is capable of operating at LAr temperature. Such capability is not available on any off-the-shelf camera models, except by chance. While visible spectrum imaging devices exist that operate at cryogenic temperatures (e.g. in astronomical telescopes), they are not standard components accessible to most consumers.

Standard electronics fail at cryogenic temperatures for many reasons, and the cause of a particular failure is rarely predictable. For even the simplest camera circuits, the 


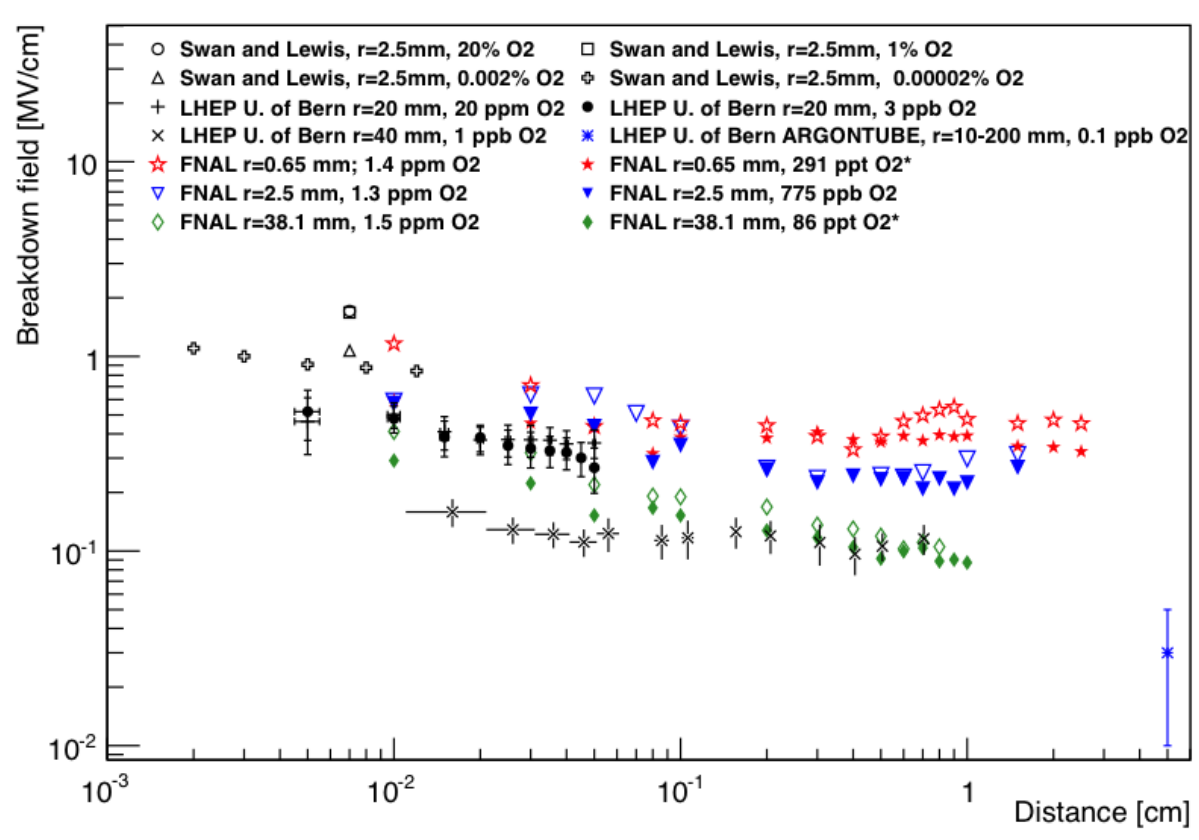

Fig. 4.1 Measured electric breakdown fields compiled from many studies [150].

electronic components include resistors, capacitors, crystal oscillators, and flash memory. Past studies $[151,152,153]$ have shown these types of components to function on their own at cryogenic temperatures, but the circuit is not guaranteed to work as a whole. Thus, it is not apparent that any particular piece of electronics will function successfully at cryogenic temperatures. It is thought that physical forces, including thermal shock, are more likely to cause failure due to damaged connections than degradation of the underlying semiconductor performance (e.g. hot carrier degradation [154]).

As part of previous unpublished work by a summer student at Sheffield, motivated by the use of digital cameras to directly detect noble liquid scintillation light, several complementary metal-oxide semiconductor (CMOS) cameras were found to function when submerged in liquid nitrogen $\left(\mathrm{LN}_{2}\right)$. These cameras happened to be small in size and electronically basic (no extra functionality besides collecting an image and transmitting it in analogue format). In particular, the most successful device was a car reversing camera, which was initially chosen for its wide angle lens, a low-temperature rating of $-40^{\circ} \mathrm{C}$, mechanical simplicity, and small size. These aspects were the basis of the search for an appropriate camera model for the 35-ton HV monitoring system.

Both charge coupled device (CCD) and CMOS cameras were considered for use in the system, the primary difference being the nature of the "shuttering" mechanism. In a CCD camera, all pixels are read out at once, leaving the entire sensor insensitive to light during the readout period. However, in a CMOS camera, only one row of pixels is read out at a time, 
leaving the other rows able to continue recording light. Therefore, the CMOS sensor was anticipated to be better suited to record short bursts of light since a large fraction of the total pixels is active at any given time.

Twelve different models of car reversing cameras were tested for cryogenic performance, some which had CCD image sensors and some which had CMOS sensors. Ten were generic unbranded car reversing cameras intended for original equipment manufacturer (OEM) use, and the other two were the high resolution, branded models Sony XAR800C and Kenwood CMOS-220. Each camera was individually tested to operate at room temperature, but only three still transmitted a recognisable image when at $77 \mathrm{~K}$. As an example, Figures 4.2, 4.3, and 4.4 show the three working cameras at room temperature and submerged in $\mathrm{LN}_{2}$. Figure 4.5 shows the Sony camera and its failure mode once cold. Of the three working cameras, OEM camera \#3 was chosen, whose characteristic warm and cold images are shown in Figure 4.4.

The chosen camera had a CMOS image sensor with $712 \times 486$ pixels, a PAL formatted video signal, a rolling shutter frame rate of 50 frames per second, 8-bit RGB colour output, and a $107^{\circ}$ viewing angle. Eight such cameras were purchased for use in the 35-ton HV monitoring camera system.

\subsection{Camera System Description}

The full multiple-camera system for installation in the 35-ton cryostat (see Figure 4.6) consisted of many different components. These include the cameras themselves, stainless steel (SS) housings for the cameras, cold and warm cables, mounting brackets inside the cryostat, DC power supply, resistive temperature device (RTD) readout, video acquisition, control software, and the interface with the rest of the 35-ton equipment.

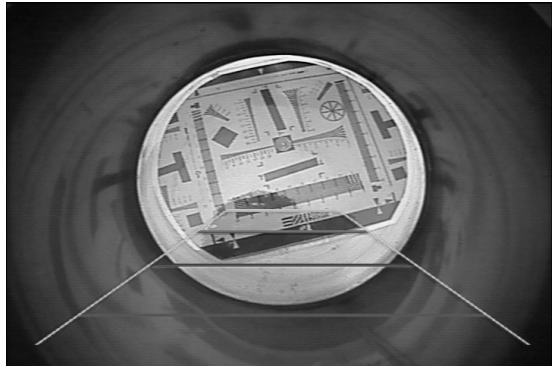

(a)

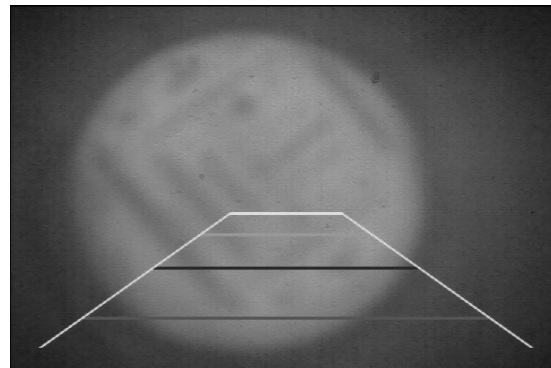

(b)

Fig. 4.2 OEM camera \#1 (a) at room temperature and (b) submerged in $\mathrm{LN}_{2}$. 


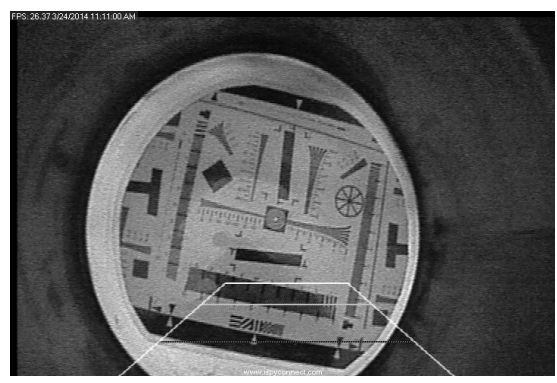

(a)

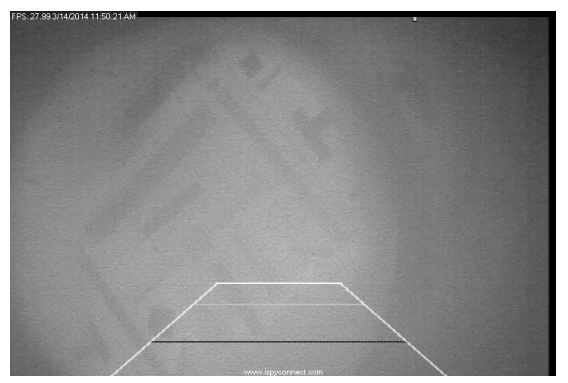

(b)

Fig. 4.3 OEM camera \#2 (a) at room temperature and (b) submerged in $\mathrm{LN}_{2}$.

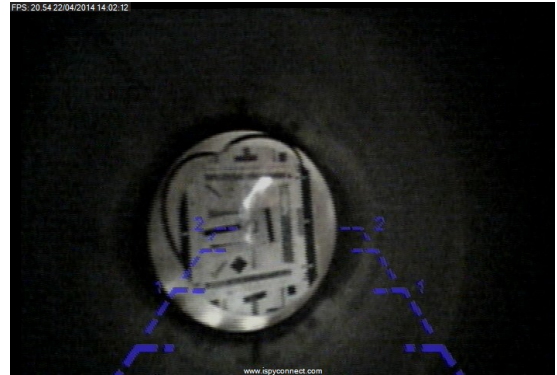

(a)

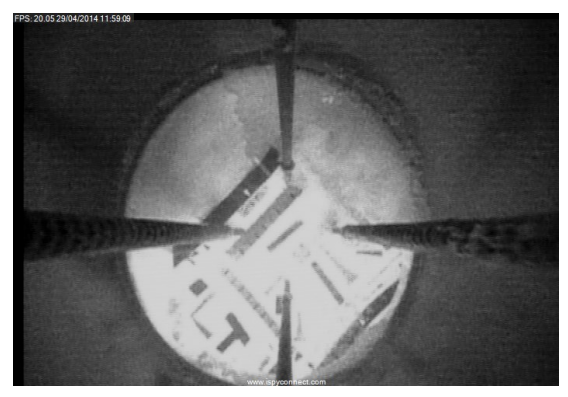

(b)

Fig. 4.4 OEM camera \#3 (a) at room temperature and (b) submerged in $\mathrm{LN}_{2}$.

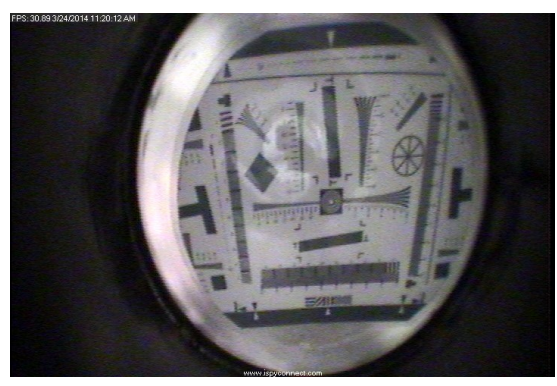

(a)

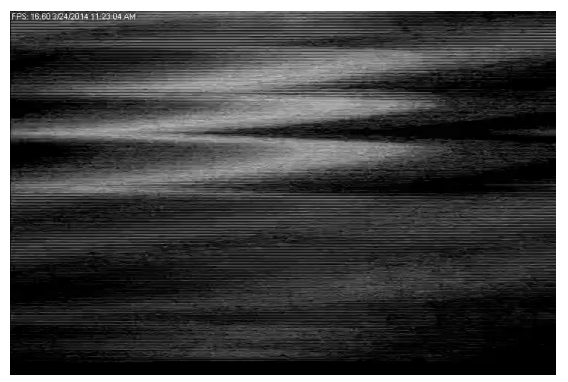

(b)

Fig. 4.5 Sony XAR800C (a) at room temperature and (b) submerged in $\mathrm{LN}_{2}$. 


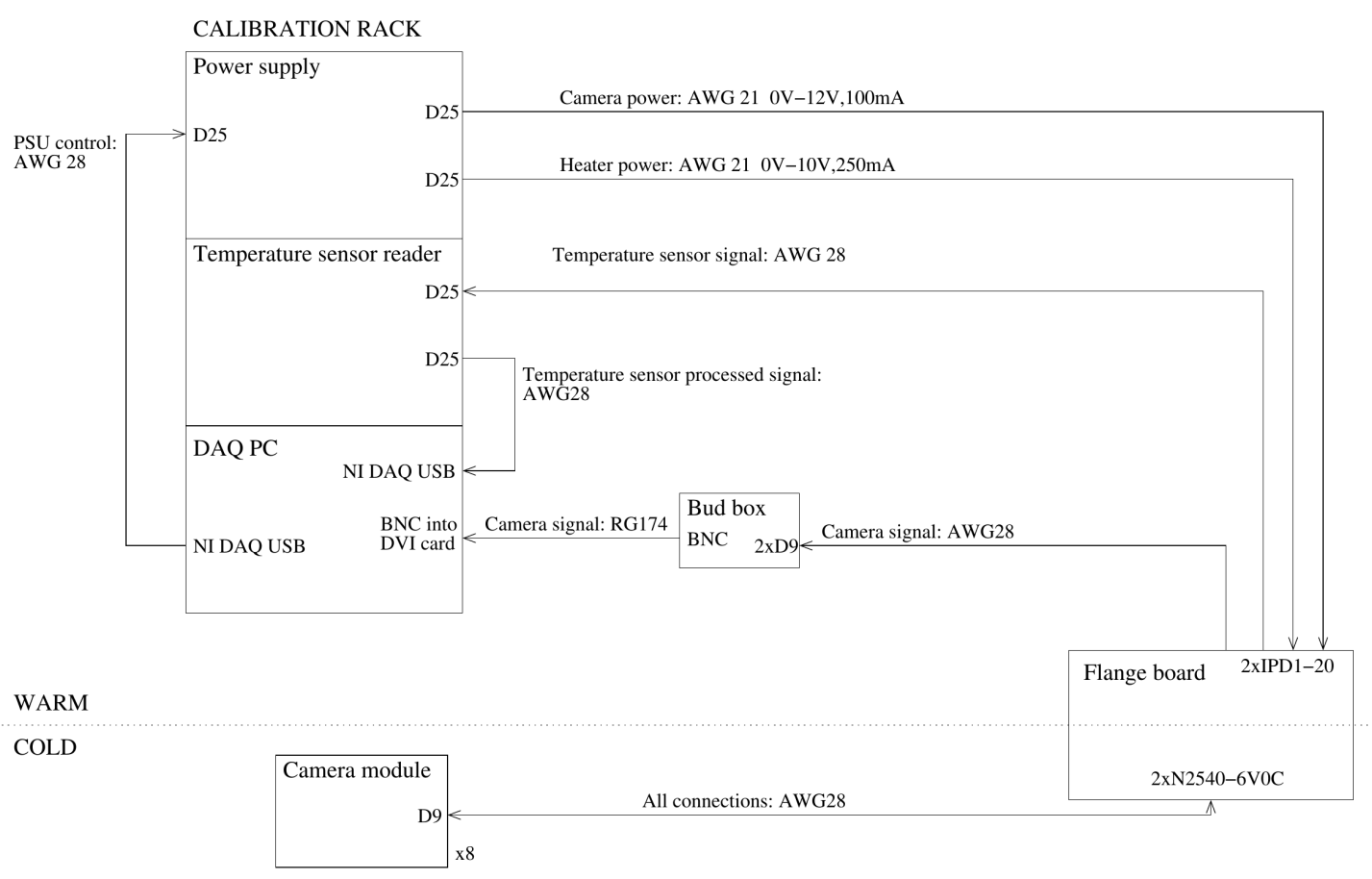

Fig. 4.6 Camera system block diagram including specific cable gauge and connector information.

\subsubsection{Camera Modules}

Since maintaining such a high level of purity in the LAr is crucial to TPC operation, the camera system must not outgas electronegative impurities to the environment. Because the cameras used were purchased from an OEM distributor, there was no control over the materials used to make up the camera packaging, and therefore it was impossible to control for outgassing of impurities into the cryostat. Thus, it was necessary for the system to be separated from the pure LAr by a vacuum-tight barrier.

The cameras were placed inside SS housings, which prevent contamination of the LAr by the camera materials and provide a robust, convenient, and easily installable system. The housing itself was made from standard Conflat-40 components (see Figure 4.7): a double-sided barrel connector, a glass window, and a 9-pin D-subminiature connector flange. All elements were held in place by a custom designed polytetrafluoroethylene (PTFE) mount which fits snugly in the barrel holding the camera lens up against the glass window (see Figure 4.8).

In each housing, the camera was mounted with two $20 \Omega$ thick film power resistors on either side to provide backup heating (more details in Section 4.4). The resistors were connected in series to be powered with $10 \mathrm{~V}$, providing $2.5 \mathrm{~W}$ of heating power. A PT100 


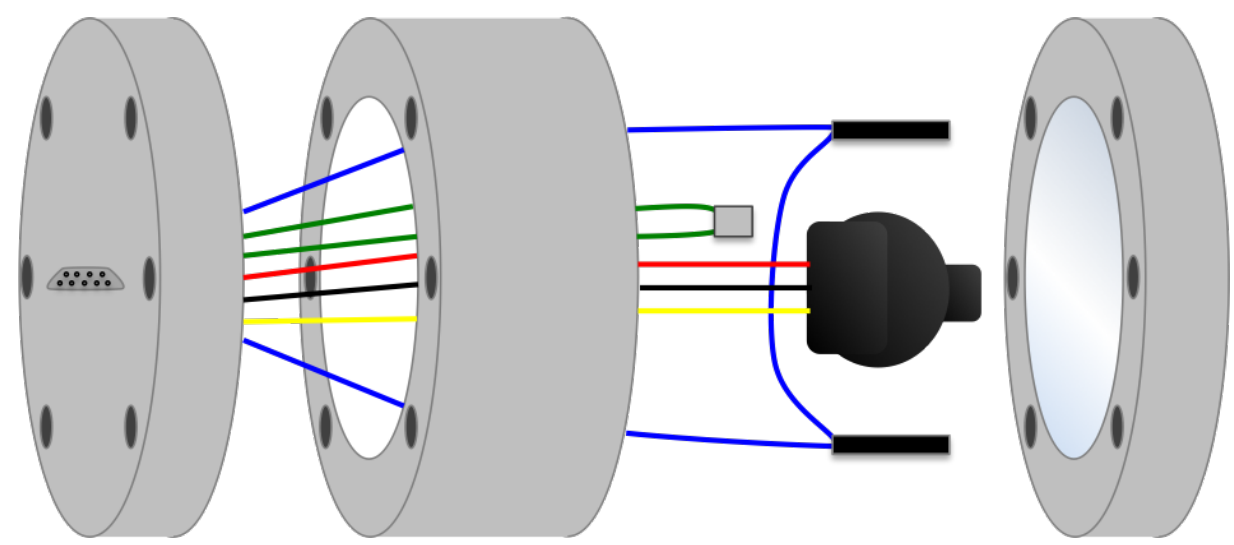

Fig. 4.7 Exploded diagram of camera and components in housing. From left to right: Conflat-40 flange with 9-pin D-subminiature feedthrough, double sided barrel connector, PT100 temperature sensor (green wires), camera centred (red, yellow, black wires), heating resistors (blue wires), flange with glass window.

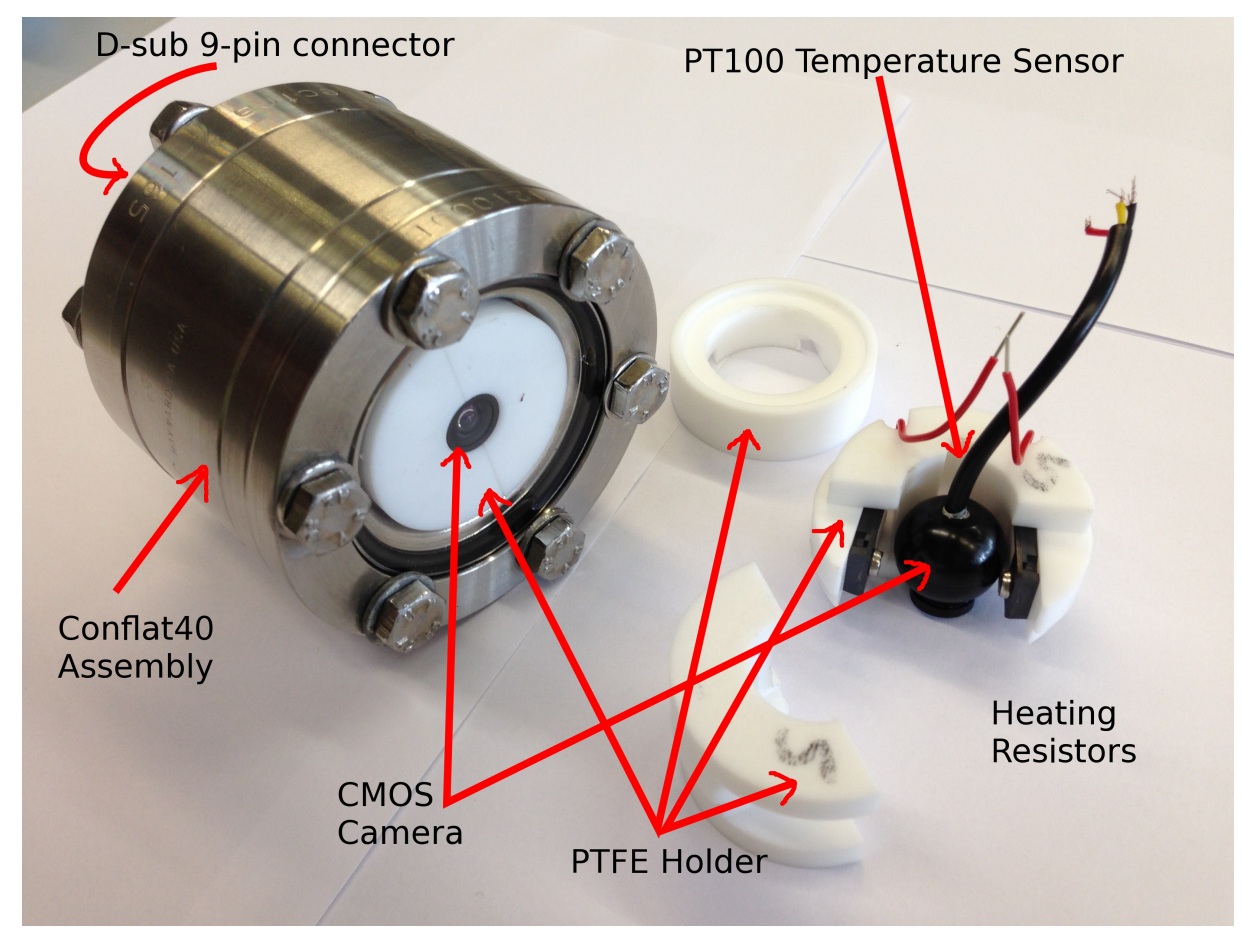

Fig. 4.8 Left: a complete, sealed camera module. Right: camera innards, pulled apart for internal component identification. 
sensor was mounted on the camera inside the housing to monitor the internal temperature. The sensor had a precision of $\pm 0.5^{\circ} \mathrm{C}$ and was connected in the four terminal configuration which was least sensitive to line resistance due to the long signal path from the modules to the readout system. Each camera module was sealed in a helium environment, and leak checked to ensure vacuum tightness. With all components, each camera module had a mass of $1.2 \mathrm{~kg}$.

\subsubsection{Mounting Bracket}

To mount the camera modules inside the 35-ton cryostat, an SS holder was designed and built (see Figure 4.9). The most straightforward place to install all of the cameras was on various cryogenic support system 3 inch diameter steel pipes. The cameras could then be mounted closer to their targets without having to weld anything, as well as having full hemispherical rotability to allow fine tuning of the field of view orientation.

\subsubsection{Cables}

To prevent contamination of the LAr through outgassing, PTFE jacketed cables were used to transmit signals from the camera modules to the electronic feedthrough on the cryostat. Each signal and return path were twisted pairs to provide shielding for the signals, as well as to protect the other sensitive electronics in the TPC from possible electromagnetic pollution from the camera signals. At several points on the path between the cryostat interior and the data acquisition (DAQ) system, the eight camera module signal and power wires were untangled for easy connections to each subsystem. The untangling of signal wires is illustrated by the connector descriptions in Figure 4.6. For example, the "bud box" converted two 9-pin D-subminiature connectors to 8 BNC sockets for secure connections to the DAQ.

\subsubsection{Power Supply}

To provide the DC power to the cameras $(12 \mathrm{~V})$ and the resistor heaters $(10 \mathrm{~V})$, a customdesigned power supply was built by the Electronics Workshop at the University of Warwick with the capability of 10 output channels for each $12 \mathrm{~V}$ and $10 \mathrm{~V}$ (see Figure 4.10). Each channel was controllable by a digital TTL pulse so each camera and heater could be individually powered on or off at any time.

\subsubsection{PT100 Conditioner}

The four-wire PT100 temperature sensors required special signal conditioning to be read out as a voltage which is proportional to the temperature. A custom designed PT100 signal 


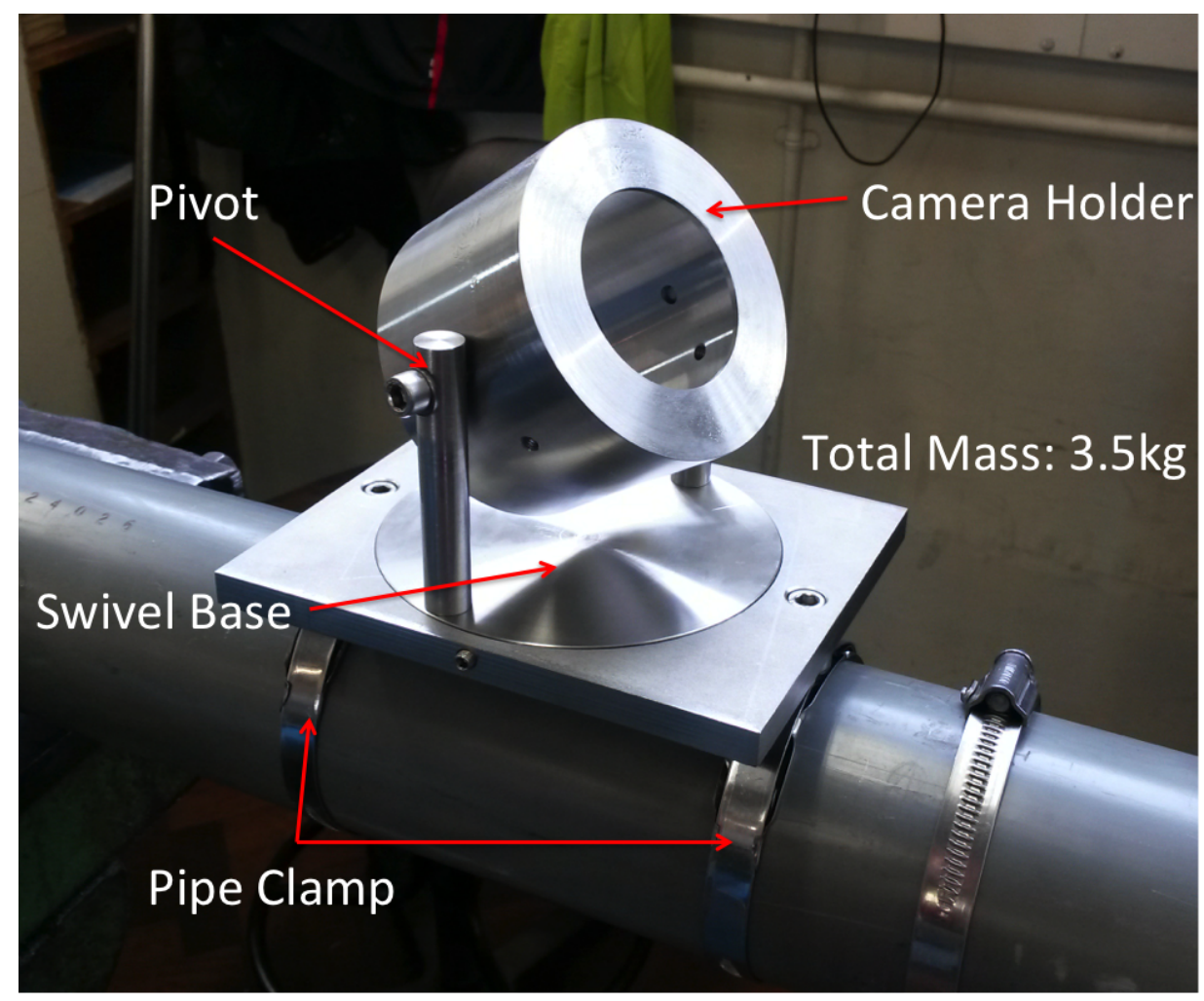

Fig. 4.9 Mounting bracket components description.

conditioner was built at the Electronics Workshop at the University of Warwick with the capability of measuring 10 four wire PT100 sensors (see Figure 4.11).

\subsubsection{Data Acquisition}

The DAQ and system control were both located in an electronics rack. The video acquisition system consisted of a digital video recorder (DVR), model Swann DVR83425H, sold as a home security video recorder. The DVR had eight channels of video recording and 1 TB of hard disk drive storage for recording videos. The system included built in triggering software which allowed automatic recording of videos and timestamps when any change above a certain threshold, including light flashes due to sparks, was detected. In addition to the auto-recording feature, live streams were available from each camera, which was used for real-time monitoring of the cooldown and ramping up the TPC HV.

All other system components were controlled via a Windows PC, which interfaced with the external temperature and power control signals through two National Instruments USB-6009 control units with voltage measurement and digital pulse output capabilities, as shown in Figure 4.6. The USB-6009 units controlled the custom power supply with digital 


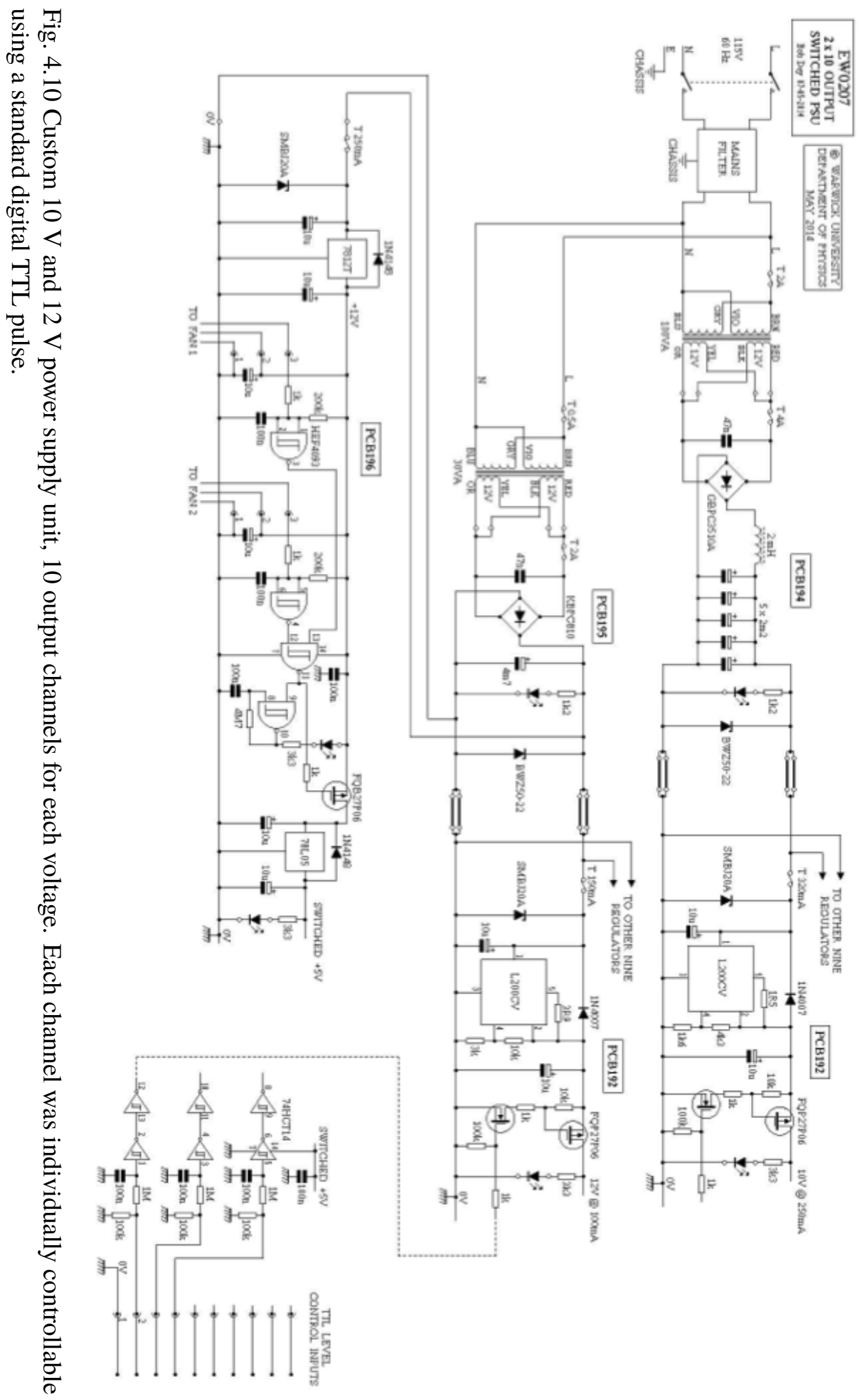




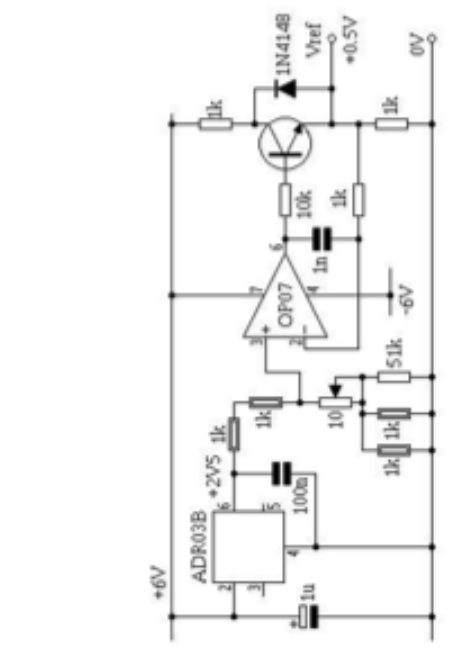

$\frac{0}{\tilde{U}}$
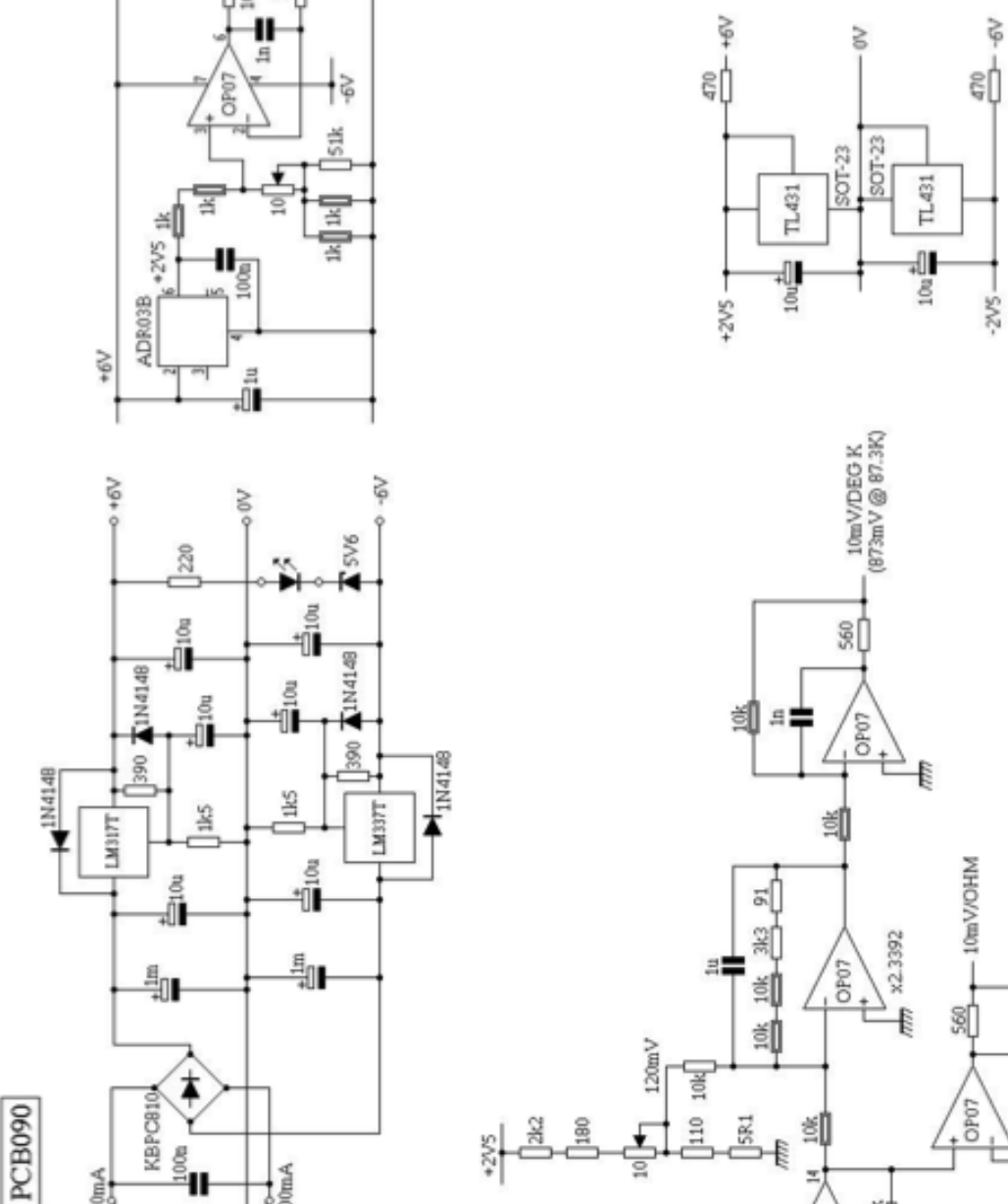

옹

政 
output signals and the custom PT100 reader by measuring analogue voltage. This subsystem was controlled with a LabView VI which provided the user interface to the whole system. The heating resistors were manually controlled, and the temperatures in each module were continually monitored and displayed via the LabView program.

The entire system had remote operation capabilities through the Remote Desktop system, which allowed direct control from any computer with a Fermilab IP address.

\subsection{Camera Performance Studies}

The behaviour and performance of the camera system when subjected to various conditions was studied before installation in the 35-ton cryostat. These studies revealed many features of the cameras and even motivated late stage system modifications (i.e. the inclusion of heating resistors).

The cameras were first tested regarding the heat load provided to an LAr system due to self-heating during normal operation. Figures 4.12 and 4.13 show how the temperature of a camera and local environmental change during camera power on and after power off without the use of the heating resistors. Ignoring slight calibration errors between identical temperature sensors, the equilibrium temperature of the camera in the LAr system was $12 \mathrm{~K}$ warmer, but the local temperature in the vicinity of the camera did not rise appreciably. The lack of temperature rise on the housing is due to the high thermal conductivity of the SS camera housing evenly dissipating the internal heat over the large surface area.

It was observed during testing that while a camera may operate at LAr temperature if powered on before cooling down, cycling the DC power source off and on again would cause the camera to fail. Once at a sufficiently higher temperature, however, the camera would revive and turn on again with no apparent permanent damage to the electronics. The cause of this was unknown, but suspected to be due to the electronics' tendency to self-heat during operation which keeps the circuit at a high enough temperature to operate successfully but would fail if the temperature were allowed to drop further by turning off the power.

While the cameras were chosen for their ability to function at LAr temperature without the aid of additional heating, due to this failure mode, a $2.5 \mathrm{~W}$ heater was included in the camera system as a fail-safe only (see Section 4.3.1). To test the heater's effect on the surrounding LAr, in particular, to check if the internal heating would cause bubbles, which could, in turn, cause electric breakdown [155], the local temperatures in and around the camera housing were measured while submerged in LAr. Figure 4.14 shows a $77 \mathrm{~K}$ increase in internal temperature following switch on of the heater, yet no appreciable change in temperature outside of the steel housing. The heat generated in the steel housing is diffused into the 


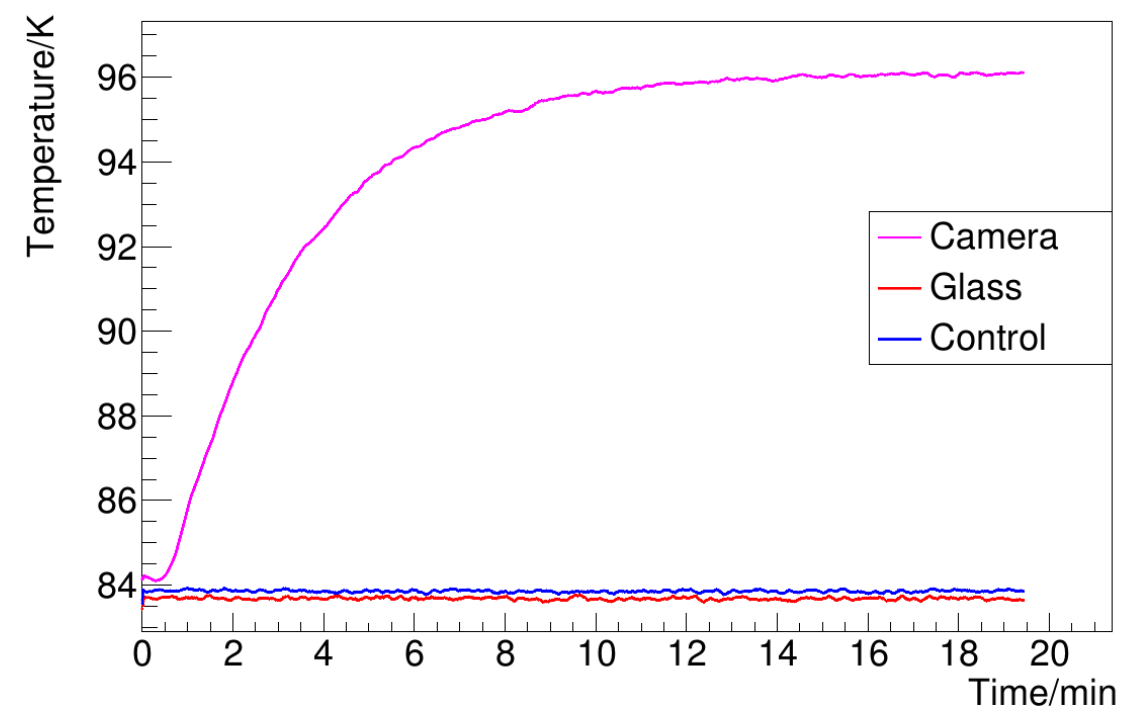

Fig. 4.12 Measurement of camera self heating from power on in LAr. Local temperature of the camera inside the housing, on the outside of the glass window, and in the LAr away from the camera housing.

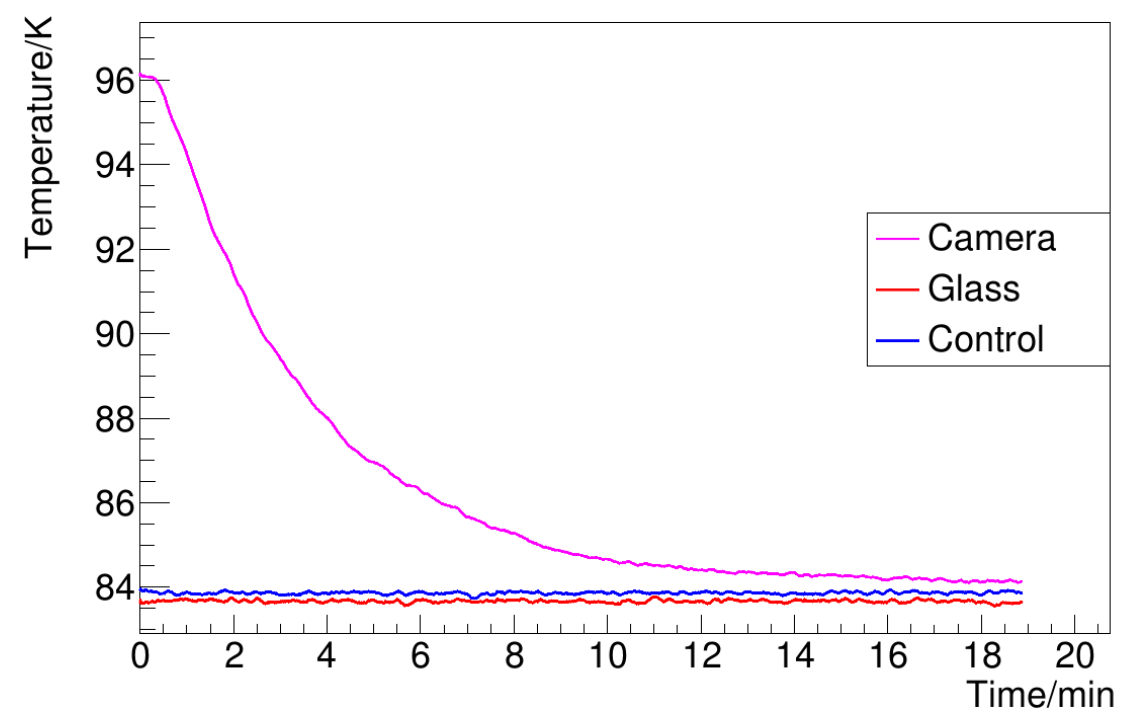

Fig. 4.13 Measurement of camera returning to ambient temperature after power switch off in LAr. Local temperature of the camera inside the housing, on the outside of the glass window, and in the LAr away from the camera housing. 
surroundings, which provides the cooling power to prevent bubbles from forming around the camera module.

The chosen working camera was then tested for changes in frame rate and voltage supply requirements between warm and cold operation. Neither the total frame rate $(20 \mathrm{~ms} /$ frame $)$ nor pixel rate was changed by cooling the camera to $77 \mathrm{~K}$. However, the camera did lose colour sensitivity.

A test of the long-term stability and operation of the camera was performed while keeping it submerged in $\mathrm{LN}_{2}$ for 96 hours continuously. No visible changes were observed in the image quality of the camera nor were any of the future operations seemingly adversely affected. More extended tests were not performed due to the impracticality of maintaining an $\mathrm{LN}_{2}$ or LAr bath for such a long period. The risk of accelerated semiconductor degradation at cryogenic temperatures is acknowledged, but without the facility to test the camera directly, the expectation remained for the cameras to operate at the same level of performance for the duration of the full 35-ton Prototype Phase II run.

The resolution of the camera is important in determining the limits of observation, given the viewing distance within the cryostat. The minimum resolving distance was measured by shining two spots of light at the camera at a camera-source distance of $10 \mathrm{~cm}$ and varying light spot separation distance. The resolution did not appear to be affected by cryogenic temperatures (see Figure 4.15) as the two spots were still differentiated at a $2 \mathrm{~mm}$ separation, but indistinguishable at $1 \mathrm{~mm}$ separation, in both warm and cold tests. However, increased optical attenuation and diffusion in LAr are observed by the reduced intensity of light recorded in the cold.

A similar test was performed at room temperature, whereby the camera-source distance varied from $10 \mathrm{~cm}$ to $2 \mathrm{~m}$, to quantify the resolution for distances similar to those in the 35-ton cryostat. In each case, the minimum distance resolvable between two features was recorded. The resolution, $\theta_{R}$, was calculated as

$$
\theta_{R}=\tan ^{-1}\left(\frac{\text { camera-source distance }}{\text { feature size }}\right) .
$$

For the chosen camera, the mean resolution across all separation distances was $\theta_{R}=2.89 \pm 0.26$ degrees.

The timing sensitivity of the camera must be known to record an image of a light-producing event in the detector. The time span between the start and end of the output light of a spark can vary depending on the distance between anode and cathode, and the total charge released in the breakdown event. The rolling shutter nature of the camera overcame this uncertainty because each pixel on the CMOS chip collected light for nearly $100 \%$ of the time (485/486, 


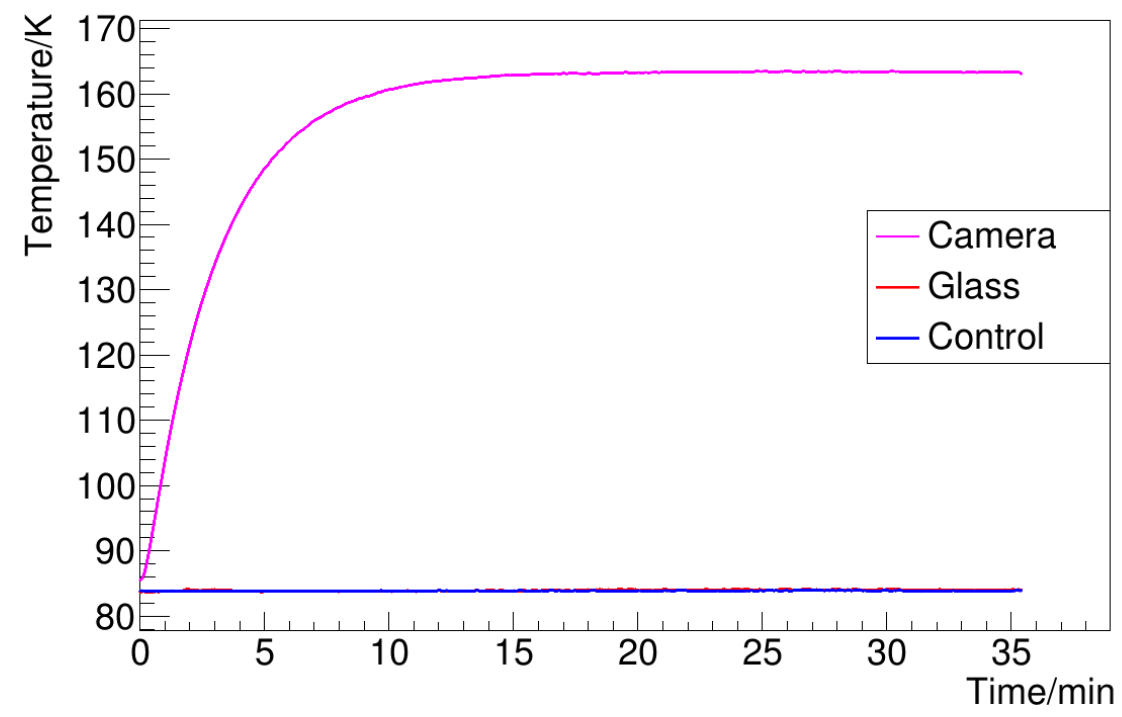

Fig. 4.14 Local temperatures in and around the camera housing following switch on of the internal heater in LAr. The line representing the "Glass" temperature is hidden behind the "Control" line.

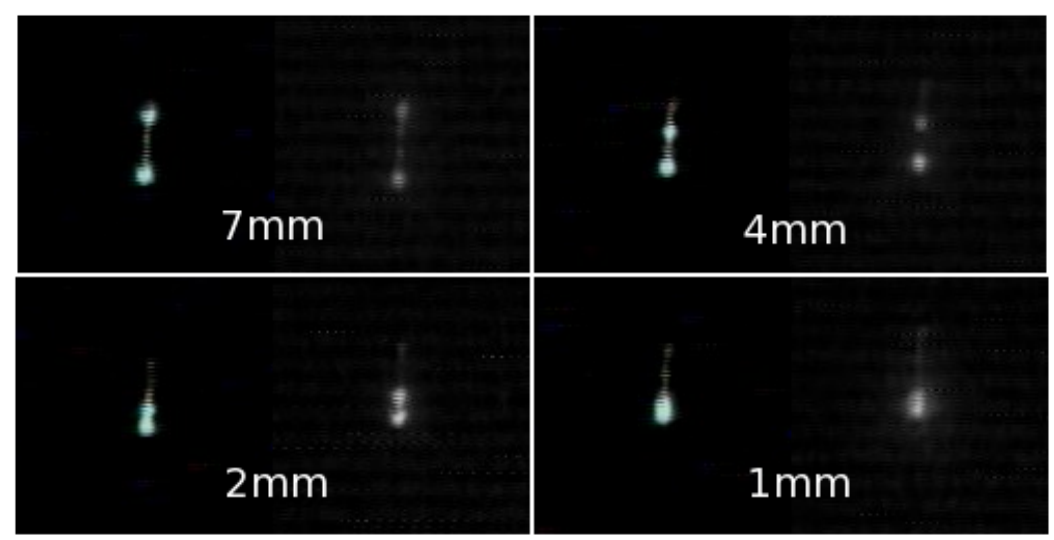

Fig. 4.15 Camera stills taken in warm (left) and cold (right) at a distance of $10 \mathrm{~cm}$ from the light source, and with light spot spacings of $7 \mathrm{~mm}, 4 \mathrm{~mm}, 2 \mathrm{~mm}$, and $1 \mathrm{~mm}$. 
to be exact), the only downtime being when the row of pixels was being read out. So, a light flash of any duration was discoverable, as long as the total light incident on the chip was above the noise threshold during that time. During testing, LED light pulses of $20 \mathrm{~ns}$ duration were consistently detected with the camera, which is a much shorter time than the expected duration of a spark light flash, $O(\mathrm{~ms})$.

To test the camera's response to a spark, an HV was applied across a printed circuit board (an anode-cathode distance of $1.4 \mathrm{~mm}$ ) until breakdown occurred both in air and submerged in $\mathrm{LN}_{2}$. Photos of the spark are shown in Figure 4.16. In both cases, the camera, located about $10 \mathrm{~cm}$ away, recorded a bright flash of light for three frames $(60 \mathrm{~ms})$ and was able to trigger the automatic recording successfully.

One significant change in the camera performance between warm and cold operation was the loss of colour encoding from the video signal in the cold. The cause of this was unknown but suspected to be a partial failure of the onboard colour digitisation circuitry (part of the PAL encoding process) in the cold. This loss of colour is seen in Figure 4.17 where the ratios between the colours have equalised, resulting in grayscale images.

\subsection{System Operation and Results}

Eight cameras were installed in the 35-ton cryostat, all outside of the TPC field region, six monitoring for $\mathrm{HV}$ breakdown on the cathode plane, HV feedthrough, and various high field locations in the cryostat. The other two cameras were installed to observe the cryogenics operation of the cooldown sprayers and the phase separator. Fields-of-view of the eight cameras are shown in Figure 4.18.

Following installation in the cryostat but before cooldown, all eight cameras were characterised at room temperature. Two sources of light in the detector provided calibration images (see Figure 4.18): a halogen light bulb provided white light similar to natural sunlight, and a set of LEDs provided red light.

During filling, the cameras were successfully used to monitor the argon liquid level [156] and continued to function when fully cooled to $86 \mathrm{~K}$. All eight cameras continued to operate for the entire run, approximately 50 days at LAr temperature. Despite several occurrences of detector hall power outages and deliberate power cycling during noise hunting, all cameras continued to function following each shutdown and power-on.

The operation of the cameras in the cold revealed degradation of image quality during initial cooldown and continuous slow degradation over the full cold operation. Even though during testing the resolution of the cameras did not noticeably change from warm to cold operation, several aspects of the picture quality degraded in the 35-ton. First, the low light 


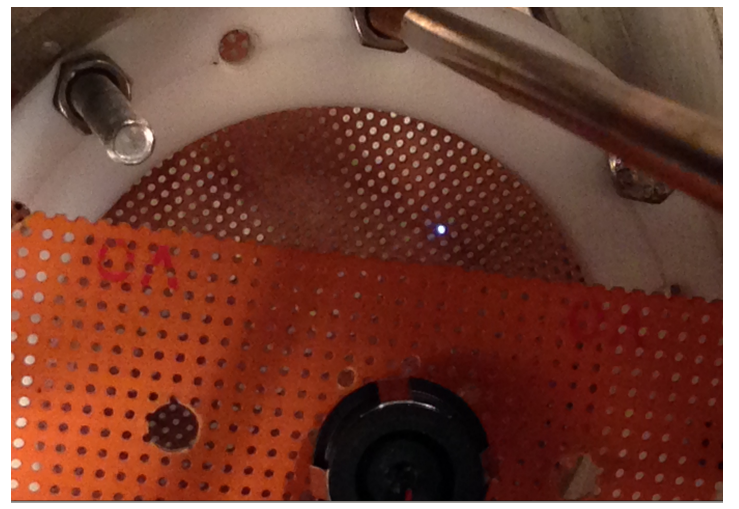

(a)

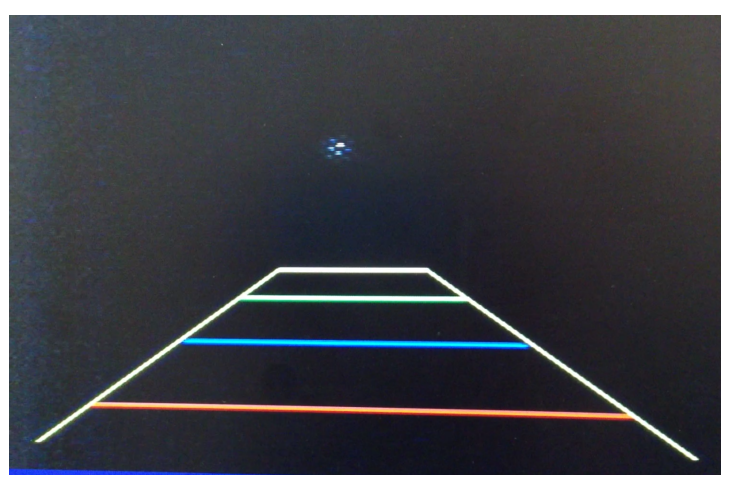

(b)

Fig. 4.16 (a) Photo of spark and equipment taken with normal digital camera. The cryogenic camera can be seen at the bottom of the image. (b) Photo of spark taken by cryogenic camera while submerged in $\mathrm{LN}_{2}$.

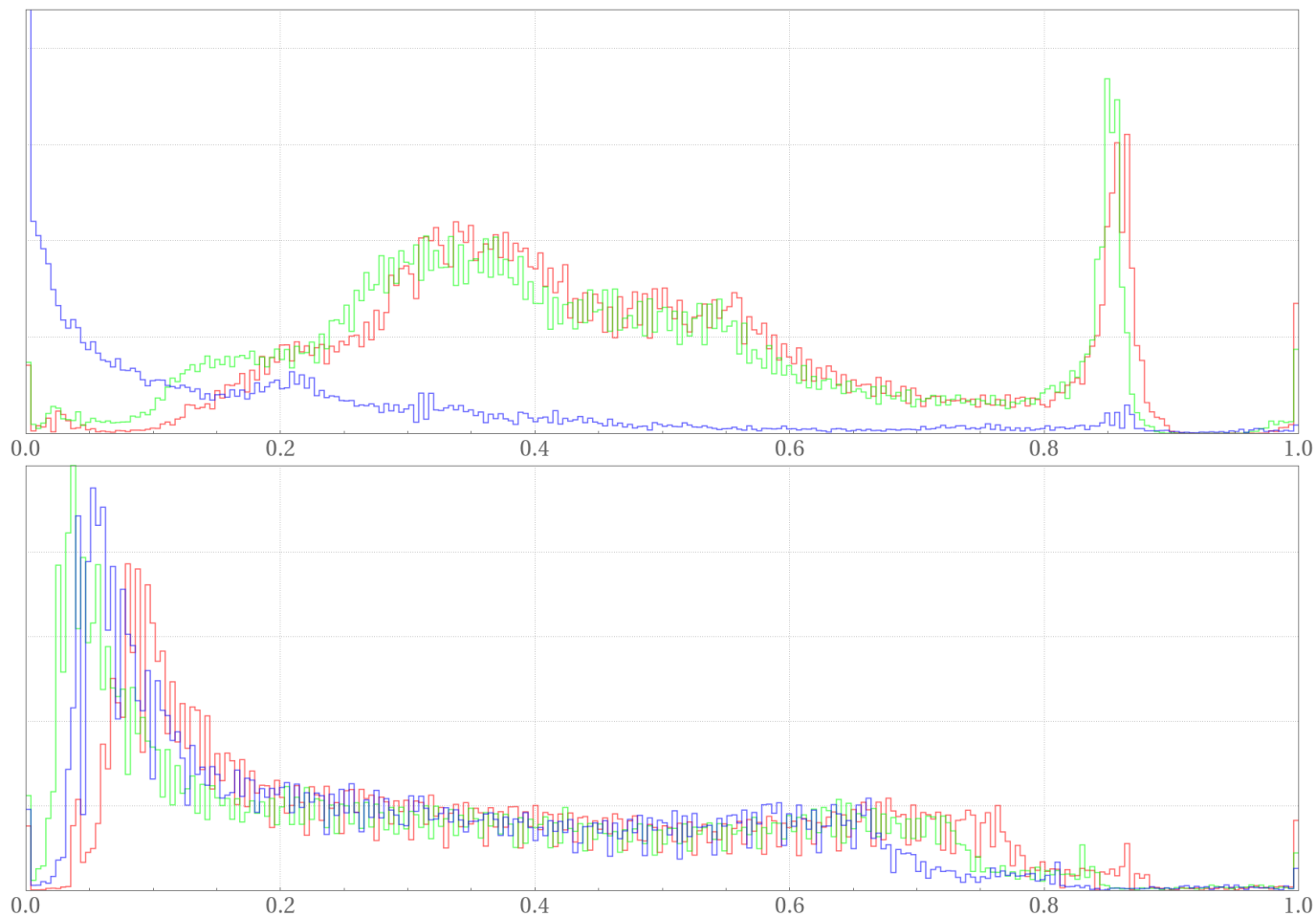

Fig. 4.17 RGB histograms of images taken before (top) and after (bottom) loss of colour encoding due to cooling of the camera to cryogenic temperature. 


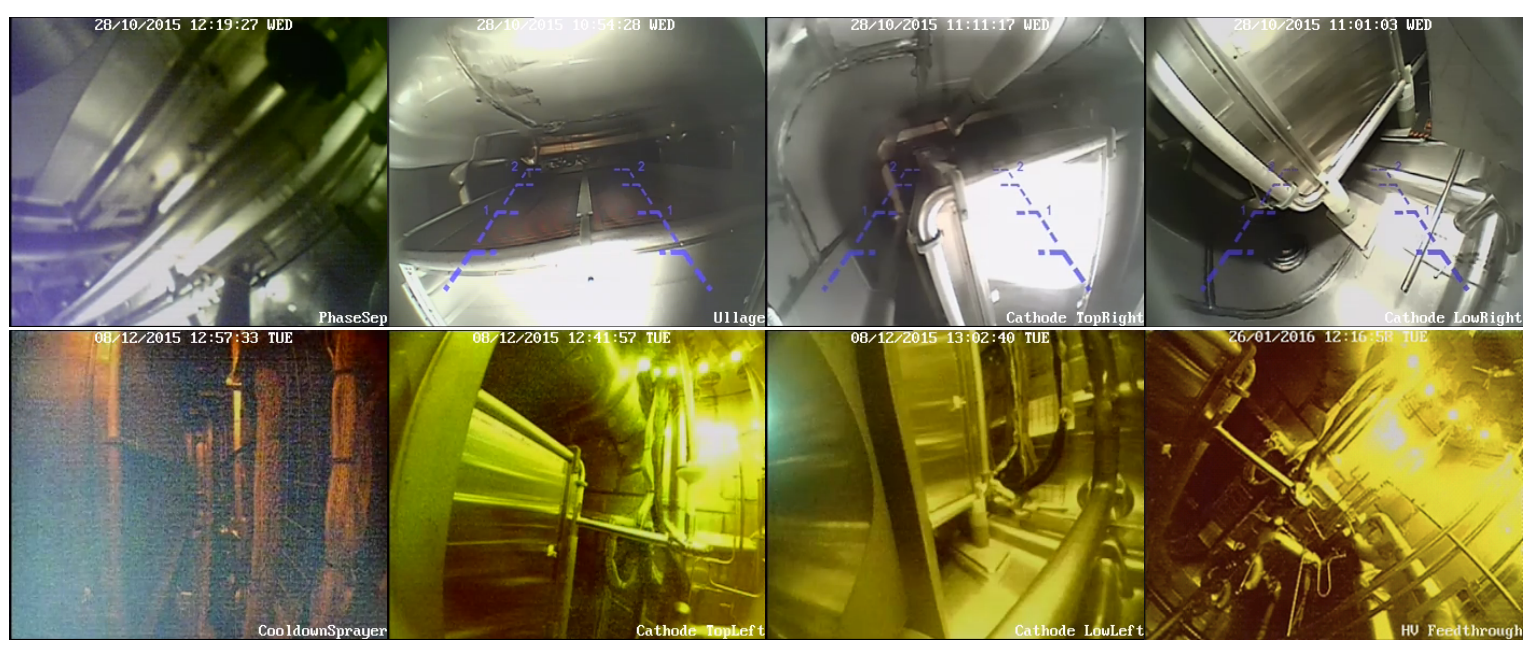

Fig. 4.18 Calibration images for system cameras. Upper (left to right): Phase separator, ullage, cathode top right, bottom right. Lower (left to right): Cooldown sprayers, cathode top left, bottom left, HV feedthrough. Top four images taken with halogen light illumination. Bottom four images taken with LED illumination. Images are left-right inverted.

level inside the cryostat led to the dominance of pixel dark-noise over possible dim signal light flashes. Also, with the monochromatic LED light source, the lack of colour depth caused various problems such as increased noise and subjectively poor image quality.

The degradation after cooldown and over time varied considerably across all eight cameras. For example, Figure 4.19 shows images taken from two cameras at three times during the experiment. It is interesting to note the smaller field of view when submerged in the liquid due to the higher index of refraction of LAr than GAr. Half of the cameras showed little post cooldown degradation, while the other half showed significant image quality issues, including increased noise, loss of colour depth, increased granularity, and loss of light sensitivity. Over the 50 day cold run, the main change in image quality was increased pixel noise rather than a degradation in resolution.

During the 35-ton Phase II run, there were six HV breakdowns detected by the HV power supply. Two happened during a period where the camera system was shut down. For the other four breakdowns, the camera system triggered and recorded video, however, no discernible light flashes were detected. There are several possible reasons for this:

- The breakdown produced visible light but did not occur inside the cryostat or in the field of view of any camera,

- The breakdown did not produce enough visible light within the camera field of view to be detected, or 


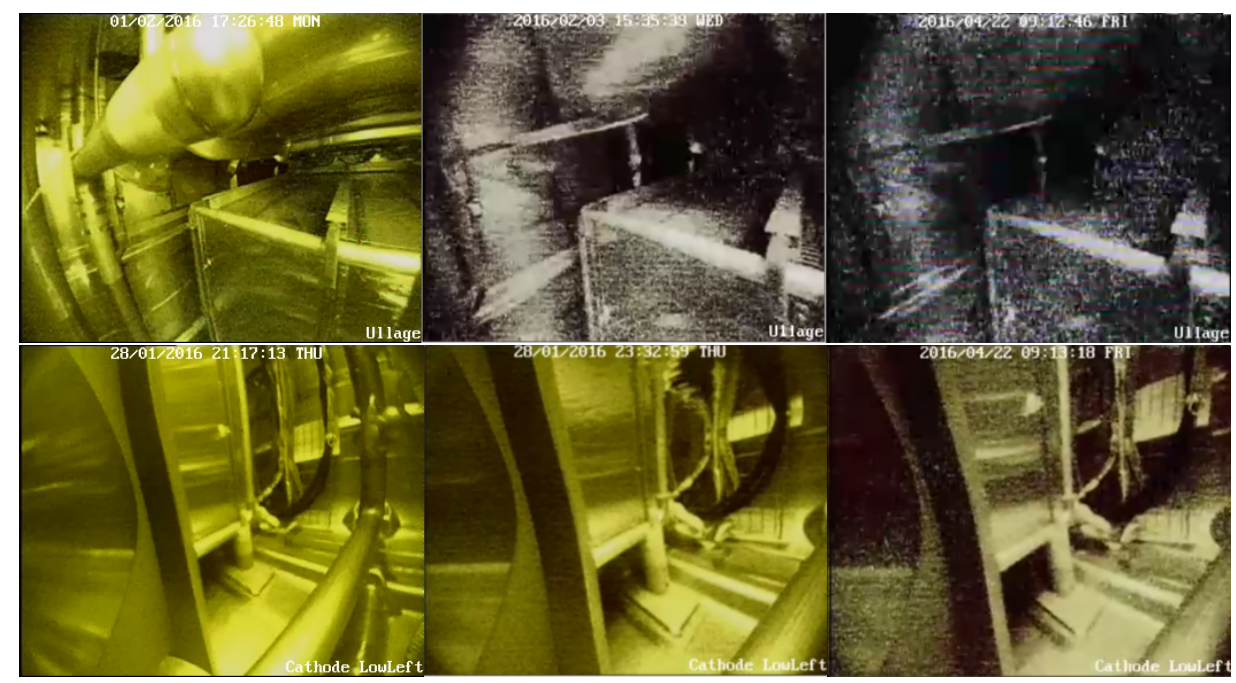

Fig. 4.19 Camera 1 (top) and camera 4 (bottom) images taken at different times. From left to right: before cooldown, immediately after cooldown, after 50 days of cold operation.

- The degradation of image quality was too significant for the detection of sparks above the pixel dark noise.

Because light-producing sparks were successfully recorded in a cryogenic liquid during testing, it is natural to assume that the cameras would have recorded video of a light-producing spark had it happened in the field of view of one of the cameras. If the breakdown occurred outside of the cryostat (for example, within a possibly damaged HV cable) the camera system would not have detected any light, but may still have triggered the automatic recording due to the electronic jitter caused by a large current injected into the common experiment ground shared by the data acquisition equipment.

After the Phase II run, a sensitive PMT was installed in the cryostat to test whether the breakdowns produced any light. If a light-producing spark occurred, it was expected that the PMT would have recorded a signal. Even if the spark occurred outside of the direct field of view of the PMT but still within the cryostat, the scattered light on the reflective cryostat walls should have produced a detectable light pulse. During the test, several HV breakdowns were recorded by the HV power supply, but no light was recorded by the PMT. Therefore, it is also possible that the breakdown events during the 35-ton Phase II run did not produce light at all.

\subsection{Summary}

A system of cameras that function at cryogenic temperatures is described and demonstrated in the 35-ton Prototype cryostat. Even though no light-producing HV breakdowns occurred 
within the field of view of the cameras, the system proved to be useful in many ways. As a first attempt to visualise the inside of a LAr cryostat during TPC operation, the system was successful. This system was the first ever camera array to be installed and successfully run in a LAr TPC cryostat without additional heating.

Future improvements are anticipated for implementation in other experiments, such as using higher quality cameras with increased resolution, less noise, and better camera-tocamera consistency in construction. It is understood that astronomical telescopes have already used cryogenically cooled CCD cameras for many years. If these technologies could be made accessible on a much smaller scale, it is predicted that major advances will be made in the cryostat monitoring systems for LAr TPCs. A long-term goal might be to use CCD or CMOS cameras inside a noble liquid detector to directly observe scintillation light. 


\section{Chapter 5}

\section{Simulation and Reconstruction of 35-ton Data}

To perform a detailed physics analysis of the argon electron lifetime in the 35-ton Prototype liquid argon (LAr) time projection chamber (TPC), especially in light of the particular running conditions of the 35-ton, the detector response and reconstruction performance must be well understood. This understanding is gained through simulating particle interactions in the TPC and analysing the performance of the reconstruction on those simulated events.

The detailed 35-ton simulation is described in this chapter, as well as the preprocessing of both simulated and real 35-ton data. Also, the hit reconstruction developed by the author is described, which is used in the argon electron lifetime measurement in the next chapter.

\subsection{5-ton Simulation}

The simulation of signals in the 35-ton TPC is performed within the existing software for LAr experiments at Fermilab. It is based on the art framework [157], which is a suite of software packages written in $\mathrm{C}++$ to assist in the processing of event-based physics data. For example, art coordinates the processing of events by incorporating software to handle parameter configuration, reading input data files, writing output files, managing data containers called "products", coordinating references between related data products, and other high-level functions as required by various experiments and analysers. It is not specific to LAr TPC experiments, nor does it perform the simulation or analysis work, which is done by the Liquid Argon Software (LArSoft) package. 


\subsubsection{LArSoft}

LArSoft [124] is a package of $\mathrm{C}++$ code, built within the art framework, which is specific to the simulation, processing, reconstruction, and analysis of data from LAr TPC experiments. In a sense, LArSoft is also a framework on which a LAr TPC experiment can build to include both shared (among all LAr TPC experiments) and specific (to the particular experiment geometry, reconstruction, etc.) code. In addition to specially developed software for these experiments, LArSoft depends on other standard packages in the high energy physics community, such as ROOT [145] and Geant4 [158]. The Deep Underground Neutrino Experiment (DUNE) specific repository, which includes the 35-ton code as well as code for the other DUNE prototypes and experiments, is called dunetpc [159]. Dunetpc contains the particular detector geometry descriptions for the 35-ton, reconstruction algorithms, data acquisition (DAQ) data processing framework (i.e. online-to-offline data formatting), and specific configurations to control various workflows and simulations. Only experiment specific software exists within dunetpc, and most of the generalised simulation and reconstruction software exists within the general LArSoft code.

The simulation of the 35-ton uses the LArSoft framework and its dependent software to create the datasets. Out of the particle generator (Section 5.1.1.1), transport simulation (Section 5.1.1.2), and detector simulation (Section 5.1.1.3) steps, only the configuration code is unique to this analysis. The data overlay step (Section 5.1.1.4) was adapted from code originally written for the MicroBooNE experiment to be relevant to the 35-ton experiment. All LArSoft readable configuration files are written in the Fermilab Hierarchical Configuration Language (FHiCL) format [160].

\subsubsection{Particle Generation}

The particle generator used in the simulation was chosen to reflect the source of cosmic particles in the 35-ton. The cosmic ray shower (CRY) generator [161] is configured to produce a single muon per event with momentum and direction sampled from a realistic parameterisation of the cosmic muon flux at the elevation of the Fermilab site. The single generated muon is produced at simulation event time 0 (rather than a random time, given expected flux, as would be the case in nature), to simulate the effect of the 35-ton DAQ event building based on a muon counter trigger. Additionally, muons are only saved if they pass through any two muon counters in the detector geometry, to simulate the real triggering scheme.

While it is unrealistic to assume only one muon occurs in the time of one TPC drift window $(\approx 3 \mathrm{~ms}$ ), generating a wholly realistic simulated cosmic muon flux is not desired 
here. Background cosmic particles, besides the primary muon, will be incorporated into the data-driven noise model, in Section 5.1.1.4 below.

\subsubsection{Particle Transport Through Matter}

The physical detector, cryostat, and experiment hall materials and geometry are described in a format which is readable by LArSoft, visualised in Figure 5.1. The simulation of particle transport through this geometry is accomplished using Geant4, which uses Monte Carlo methods to perform the simulation, and uses the theory set out in section 1.4. The FHiCL configuration given to this step defines the simulated electron lifetime.

\subsubsection{Detector Simulation}

The purpose of the detector simulation is to reproduce the impact of the particle interaction on the detector components and the signal generation. This step simulates the ionisation charge drift in the electric field and the effects of detector properties including diffusion, electron-ion recombination, and charge attenuation due to impurities, as defined by parameters in the previous simulation step. Also, the wire signals are created by the convolution of the field response, electronics response, electronic noise, and the charge impacting the particular wire signal.

For this analysis, no noise is added to the simulated signals. Because of the particular noise characteristics of the 35-ton, none of the artificial noise models included in the LArSoft package is sufficient to reproduce effects such as stuck bits, coherent noise, and run-to-run fluctuations in baseline and root-mean-square (RMS) noise, so a data-driven method of noise addition is developed.

\subsubsection{Data Driven Noise Overlay}

To better model the 35-ton noise, a data-driven noise addition model was incorporated into the signal simulation. Rather than attempting to reproduce the noise characteristics of the 35-ton data run artificially, actual data waveforms were merely overlaid onto the simulated wire signals.

The source of noise information was real 35-ton data waveforms. The same list of runs used in the data analysis (described in Section 6.1.3) was the source of input noise. The actual noise was taken from a subset of the full 15000 time-to-digital converter (TDC) tick waveforms output by the event builder. Each full waveform is structured as follows:

- Ticks 0-5000 - Pretrigger background noise, 


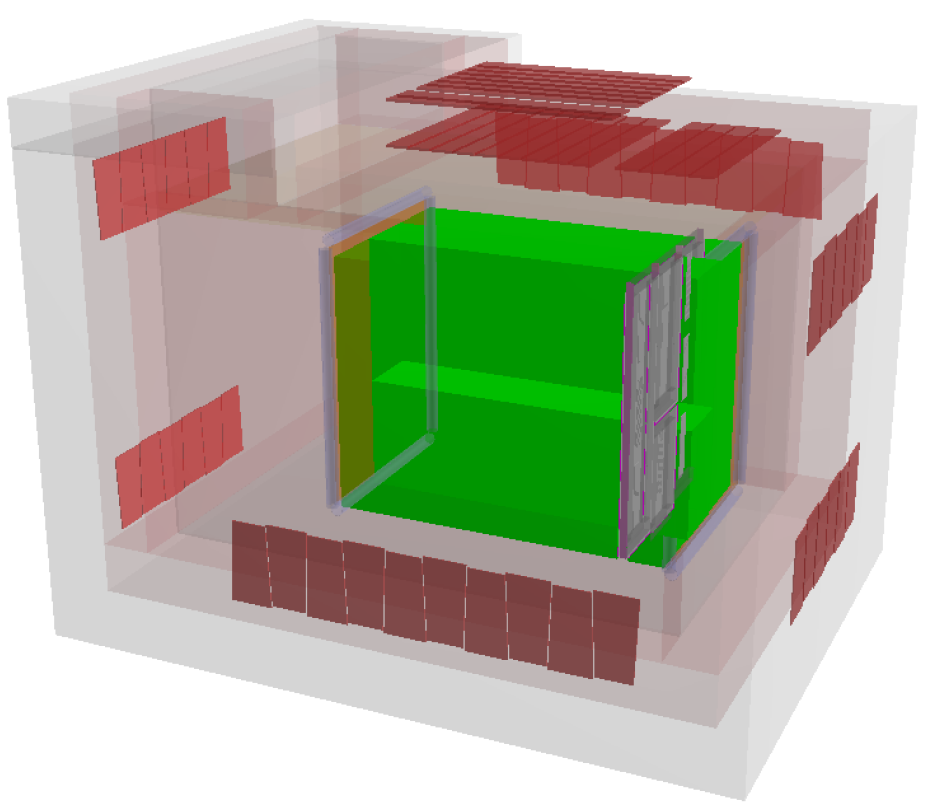

Fig. 5.1 35-ton detector geometry visualisation including cryostat, modular TPC, and muon counters.

- Ticks 5000-9024 - Single drift window, assuming drift velocity of $0.109 \mathrm{~cm} / \mu \mathrm{s}$, with muon counter coincidence trigger at tick 5000; data which are analysable,

- Ticks 9024-15000 - Posttrigger background noise.

Ticks 9800-15000 of each data waveform are guaranteed to be independent of the signal from triggered particle interactions, accounting for possible uncertainties in the drift velocity due to electric field fluctuations or timing offsets. These 5200 ticks of each waveform are used as the source of noise in the data-driven model, henceforth called "source noise".

Along with the electronic noise, the presence of background (untriggered) particle tracks which occur in these source noise waveforms is acceptable because they reflect possible backgrounds expected in any given real data waveform. Additionally, dead or missing channels in the data are not used in the simulation, accurately reflecting the varying run characteristics of the 35-ton.

The waveforms from the detector simulation stage ("source signal") are combined with the source noise waveforms using a scaled tick-by-tick addition. The simulated signal waveforms are scaled by a factor $\mu$, which represents a relative multiplier on the real signal-to-noise ratio $(\mathrm{S} / \mathrm{N})$ of 35-ton data, to simulate the effects of different possible levels of $\mathrm{S} / \mathrm{N}$. For example, $\mu=2.0$ represents twice the $\mathrm{S} / \mathrm{N}$ of the 35 -ton data, or in other words, twice as much signal. 
Further consideration of the $\mu$ parameter reveals the question of why it is not applied to the source noise analogue-to-digital converter (ADC) units since the desired outcome is a change in the effective $\mathrm{S} / \mathrm{N}$ of the simulated data. The reason for this is that the background particle tracks present in the source noise waveforms are not scalable relative to the electronic noise in the same waveforms. Therefore, to maintain the impact of background cosmic tracks on the event reconstruction, scalings are applied to the simulated signal waveforms only.

The main shortfall of this data-driven noise model is the treatment of stuck bits, summarised in Table 5.1. Given the two input waveforms, whether or not a source noise ADC is stuck, the addition of non-signal ADCs from the source signal waveform is accurate as in real data, as the non-signal ADCs are necessarily zero (since no noise was added during the detector simulation). Similarly, whether or not an ADC in the source signal is in the region of interest of a simulated particle interaction signal, the addition of non-stuck source noise ADCs will produce a well modelled summed ADC. However, if a stuck ADC from the source noise waveform is added to a simulated particle signal ADC in the source signal waveform, the resulting overlaid ADC has an error which is non-physical as the stuck source noise ADC does not accurately model the actual noise waveform.

There are many ways to deal with the issue of overlaying stuck bits, including keeping either source ADC and ignoring the other, adding the two source ADCs regardless of the stickiness of one, or devising a statistical method whereby simulating the stuck bits based on probabilities determines what the final output ADC should be. Of the methods considered, and taking into account computational speed considerations, the process where both ADCs are added regardless of stickiness was implemented. The reason this technique was chosen was that the apparent nature of the probability of any given ADC to be stuck in real data waveforms. For example, real signal ADCs which are closer to the nearest multiple of 64 are observed to be more likely to be stuck than those further away. Therefore, by adding a non-zero ADC from the source signal waveform to a stuck ADC in the source noise will likely push the simulated true signal further away from a stuck value, and in real data, may unstick the ADC anyway. These probabilities are not well understood in the 35-ton data, and therefore stuck bits have not yet been accurately reproduced in a simulation. While not perfect, this ADC addition scheme was maintained in this analysis.

Table 5.1 Accurate $(\boldsymbol{})$ or inaccurate $(\boldsymbol{X})$ simulated output waveform when ADCs of each type are added from both sources.

\begin{tabular}{r|c|c|} 
& Stuck Bit & Non-Stuck Bit \\
\hline Signal Region & $\boldsymbol{X}$ & $\checkmark$ \\
\hline Non-signal Region & $\checkmark$ & $\checkmark$ \\
\hline
\end{tabular}




\subsection{Signal Processing}

The 35-ton data were collected and stored during the run as described in Section 3.2.4 and the simulated waveforms were produced as in Section 5.1. Before detector characterisation studies or physics analyses were carried out, all waveforms were preprocessed, cleaned, and optimised. The standard 35-ton signal processing involved filtering of electronic noise, removal of bad or dead channel signals, mitigation of ADC artefacts, and deconvolution of signal shape and detector response. Real 35-ton data and simulated TPC signal waveforms were preprocessed in the same manner to minimise the systematic differences.

\subsubsection{Bad Channels}

During construction of the anode plane assembly (APA) frames, it was discovered that several wires had broken during the cooldown testing process. These wires were glued across a break between adjacent printed circuit boards so, during cooldown, thermal contraction caused the wires to break as there was no freedom given to move in response. Because they were glued, the wires maintained the original tension over the main part of the APA frame. However, an electrical connection was no longer present. The broken wires were jumpered to adjacent wires to maintain the electric field uniformity across the planes, which had the effect of making the signals recorded on the non-broken jumpered wires to be larger and more distorted than they would have been otherwise. Thus, without fully understanding the consequences on the signal shaping (future studies are planned), it was decided to remove both the broken and jumpered wire signals from the offline analysis.

For a very similar reason, wires located on the edges of each TPC were ignored. Gaps between TPCs and between the TPCs and field cage caused distorted electric fields. This design feature was implemented in the 35-ton to study the effects of the modular TPC design, as is inherent in the DUNE far detector design. The motivation for this was the desire to reduce charge losses in these regions where it is difficult or impossible to read out drifting charge. Dedicated studies are intended to understand these effects fully. However, the distorted field shaping in these regions causes unknown biases in the measured charge on these channels, and they are therefore ignored for this analysis.

As described in Chapter 3, the electronic noise throughout the detector varied significantly from run-to-run, event-to-event, and even channel-to-channel. For various reasons, channels which had unusually high noise, or particularly distorted signal, were deemed to be "bad". These channels were identified by the collaboration [162] using several metrics to determine the badness. The most straightforward of these is a cut on the baseline subtracted RMS of 
wire noise. Wires which consistently had RMS greater than 35 ADC units were ignored from further analysis.

Another metric for determining bad channels was the fraction of TDC ticks which were affected by stuck bits (Section 3.4.2). If more than $30 \%$ of all ADCs in any given waveform were identified as stuck, then the channel was removed.

During the Phase II run, the stability of simultaneously operating and reading out all 2048 wire signals from all 16 Reconfigurable Computing Elements, each reading out a single front end motherboard (FEMB), was poor. At any given time, several FEMBs had either failed or were turned off, each one responsible for reading out 128 channels. This caused significant interruptions in the reconstruction of events, as there were substantial contiguous regions in the TPC which were effectively uninstrumented.

Lastly, the remaining wire waveforms were manually inspected in the early stage of the detector commissioning to identify cases where it was obvious that the channel was dead. For example, wires may have had a reasonably low noise level and small fraction of stuck bits, but no signal pulse when adjacent channels had obvious signal pulses. Identification of bad channels using this method was conservative in that only the most obvious problems led to the channel being designated as bad.

The combination of all of these bad channel identification schemes and causes led to a minimum overall $26.5 \%$ of all channels to be unusable, including $29.2 \%$ of all collection plane wires. Channels which spontaneously appeared with a particularly high or low noise RMS in a given event were also ignored on an event-by-event basis.

For each data event, the list of bad channels varied depending on the particular noise and run conditions at the time. So, the same run based bad channel list was applied to simulated samples according to the data run which was used in the data-driven noise overlay step (Section 5.1.1.4). Therefore, the simulated samples contained only TPC channels which were used in the real data analysis.

\subsubsection{Pedestal Subtraction}

In the front end analogue-to-digital conversion of TPC signals, it was necessary to add an artificial voltage pedestal to prevent saturation given the bipolar nature of the noise and signal pulses. In the offline analysis, however, it was more convenient for the channel to have a baseline of 0 ADC units, and because negative ADCs could be more easily dealt with in software than in hardware. These pedestals were assumed to be constant over the course of several hours of real time, so every eight hours or so during the Phase II run, a "pedestal run" was recorded in which the median ADC value for every channel was stored in 
a database. During the preprocessing step of the data analysis, this database was accessed, and the pedestals subtracted from every waveform.

Because the data-driven noise addition model added real data ADCs, the resulting simulated samples contained pedestal-added waveforms specific to the data noise runs used. Therefore, the simulated waveform pedestals were subtracted in the same way as real 35-ton data.

\subsubsection{Stuck Bit Mitigation}

After identification of stuck bits in the data waveforms, an attempt was made to mitigate the effects of this phenomenon using software in the preprocessing step [163] (see Figure 5.2). The mitigation process was based on an interpolation of the true ADC values based on adjacent unstuck ADC values. The interpolation was limited to a maximum of five consecutive stuck ADCs. Otherwise, it might be possible to add features to the data which did not exist previously. The same method for mitigating stuck bits was applied to simulated waveforms as they contained real 35-ton stuck bits from the noise addition step.

\subsubsection{Coherent Noise Subtraction}

Coherent noise across 35-ton channels which shared the same voltage regulator was subtracted using a simple method. Tick-by-tick, the median ADC value was calculated for all of the channels connected to the same FEMB, then subtracted from all channels for that tick. This algorithm was implemented in the LArSoft framework for standard 35-ton data reconstruction [164]. This noise removal was effective in removing coherent noise from the data (see Figure 5.3). However, it caused some undesirable side effects. Most significantly, if there were real signal pulses at the same time on the related channels, the coherent noise subtraction would subtract out the real signal. The destructive filtering of the signal was not an uncommon case, as many triggered tracks were parallel to the APA plane, causing signals to exist at nearly the same times across adjacent channels. Because the current analysis requires exceptionally accurate ionisation charge measurement, regardless of track angle, this standard 35-ton signal processing step was not applied to data or simulated waveforms in favour of less destructive noise suppression methods.

\subsubsection{Deconvolution}

The purpose of deconvolution is to remove the effects of field and electronics response from the wire signals, and to recover the number of ionisation electrons which arrived at the readout 


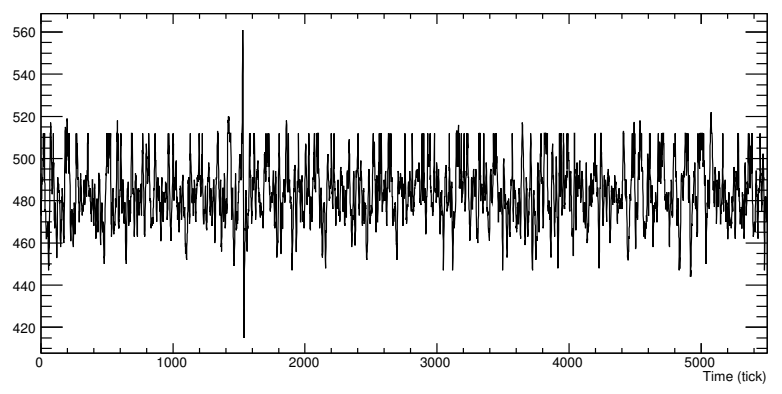

(a) Raw waveform before stuck bit mitigation.

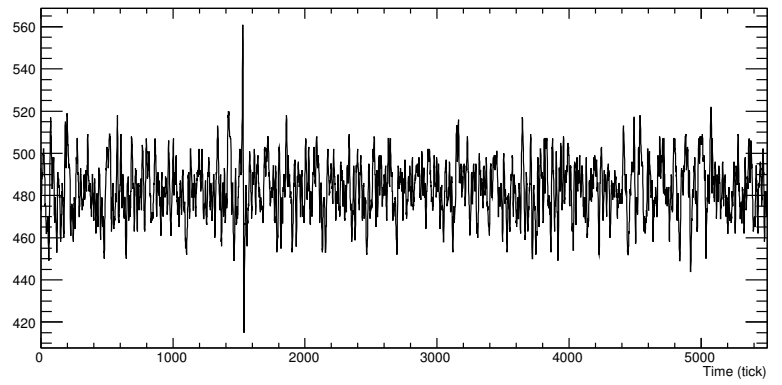

(b) Waveform after stuck bit mitigation.

Fig. 5.2 Example of stuck bit mitigation performance.

plane. Within LArSoft, this deconvolution process was implemented for the ArgoNeuT experiment [165] and adapted for the particular electronics parameters used in the 35-ton.

The field response function of the detector was determined by simulating the electric field and drifting electrons through the induction planes and toward the collection plane. The response of the channels to the drifting charge is a function of drift time and the electric field itself. The simulated response function is defined separately for each of the three readout planes, as shown in Figure 5.4 for the 35-ton.

The electronics response function was determined by the shaping and gain parameters of the preamplification and digitisation steps. Figure 5.5 shows the response function for different possible values of shaping time. For the 35-ton data run, a $3 \mu$ s shaping time was used. The gain of the preamplifier is reflected by the amplitude of the plot, which in Figure 5.5 is $4.7 \mathrm{mV} / \mathrm{fC}$, but for the 35-ton data run, was $14 \mathrm{mV} / \mathrm{fC}$.

The physical convolution of the response function $R\left(t, t_{0}\right)$ with the real signal $S(t)$ results in the measured signal,

$$
M\left(t_{0}\right)=\int_{-\infty}^{\infty} R\left(t-t_{0}\right) \cdot S(t) d t .
$$




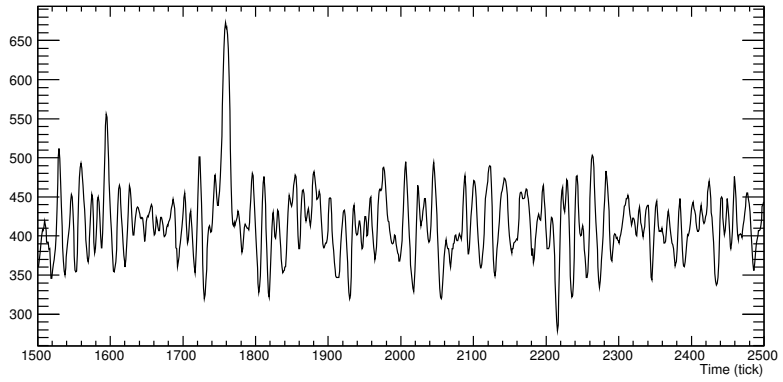

(a) Waveform after stuck bit mitigation but before coherent noise subtraction.

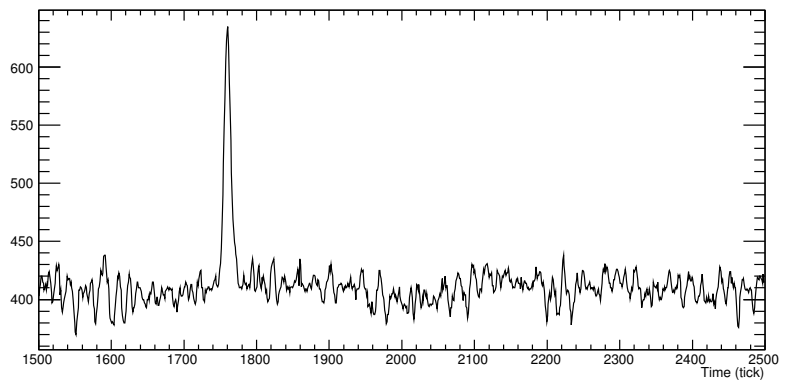

(b) Waveform after stuck bit mitigation and coherent noise subtraction (best case scenario).

Fig. 5.3 Example of coherent noise subtraction performance.

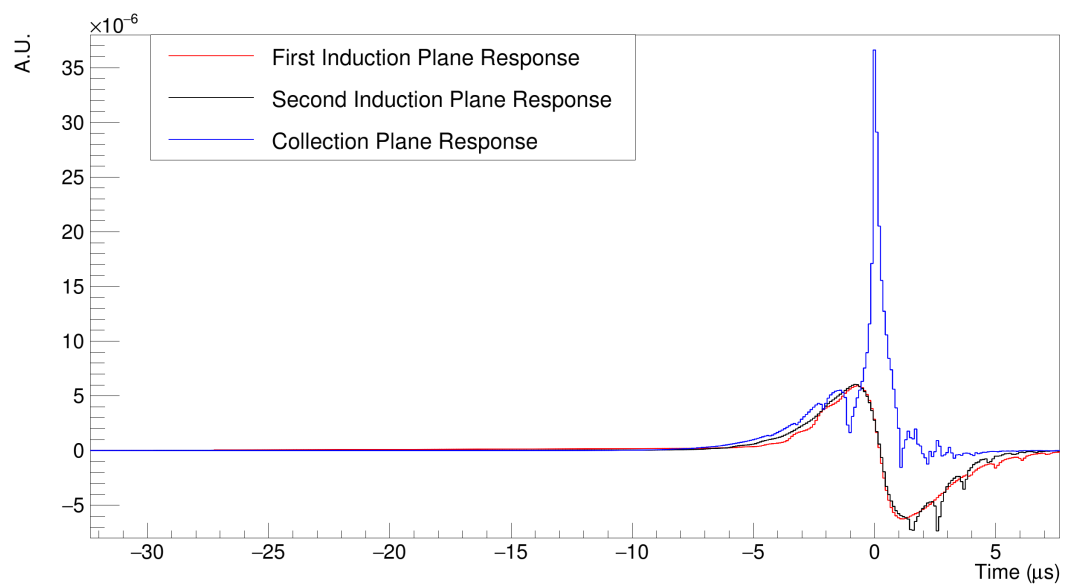

Fig. 5.4 Simulated field response of three wire planes. 
Electronics Response Function in Time Domain

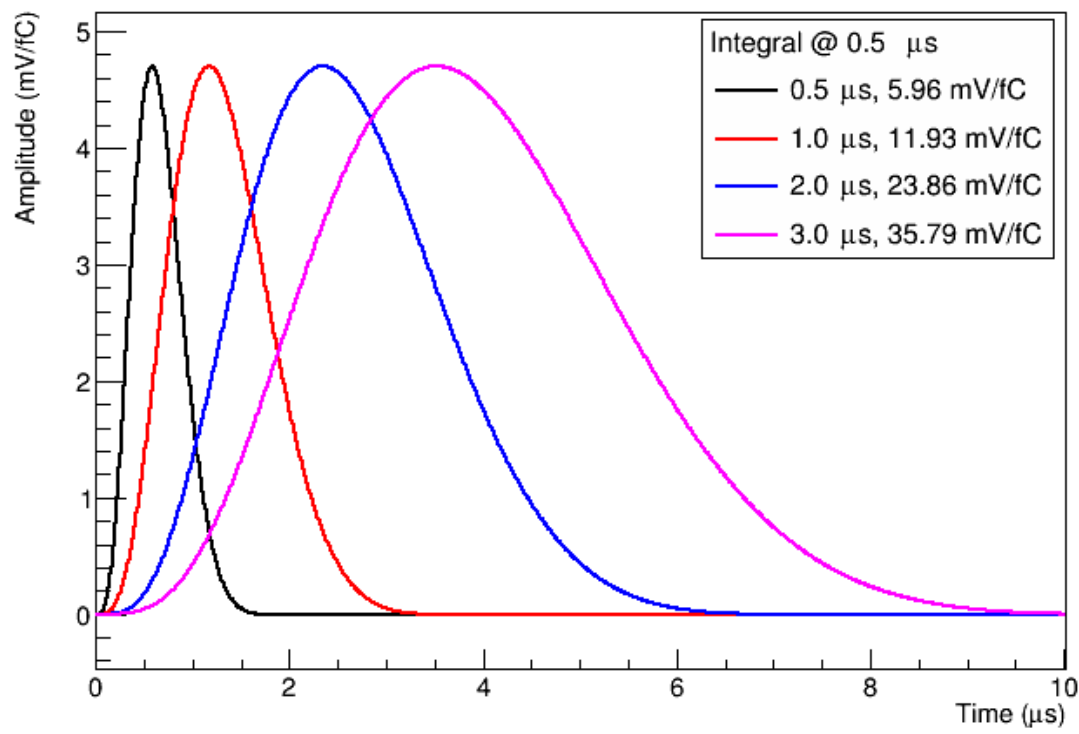

Fig. 5.5 Theoretical electronics response curves from delta pulse input at time 0. Amplitude shown here reflects gain setting of $4.7 \mathrm{mV} / \mathrm{fC}$.

Performing a Fourier transformation on both sides of the equation gives the equation

$$
M(\omega)=R(\omega) \cdot S(\omega)
$$

where $\omega$ is the frequency. Then a simple division of the measured signal by the response function (both in the frequency domain), and subsequent inverse Fourier transformation, can recover the true signal in the time domain. According to the Shockley-Ramo theorem (Equation 2.2), the induced current on the wire is proportional to the total charge of the incident electron cloud, its velocity, and the electric field in the direction of the velocity vector. Therefore the signal shape parameters extracted from the deconvolution gives directly the total number of electrons which act on a wire.

By introducing a filter function, $F(\omega)$, in the Fourier domain,

$$
S(\omega)=\frac{M(\omega)}{R(\omega)} \cdot F(\omega)
$$

the effects of electronic noise can be incorporated to get an improved estimator for the true signal, $S(t)$. For the 35-ton, a sigmoid filter function of the form

$$
F(\omega)=1-\left(1+e^{-(\omega-a) / b}\right)^{-1}
$$


was implemented [166]. The parameters $a$ and $b$ were determined by fitting the function to the frequency distribution of a wire signal. This filter function was chosen to allow low frequency signals (i.e. ionisation charge pulses on collection plane wires) and suppress high frequency noise components.

In practice, the Discrete Fourier Transform [167] is used to deconvolve the field, electronics, and noise response from the true signal since the measured signal was sampled at a fixed frequency of $2 \mathrm{MHz}$.

Deconvolution affects pulse integral and amplitude in a non-linear fashion, therefore the process could introduce an unwanted bias when the true ionisation charge is desired. Even considering the benefits of deconvolution (i.e. higher signal-to-noise ratio, better pulse timing, etc.), the bias introduced has a much more significant impact on the analysis because accurate charge reconstruction is necessary (implications of this non-linearity are explained in more detail in Section 5.3.1). For these reasons, the deconvolution of detector and noise effects is not applied to either real data or simulated waveforms.

\subsection{Hit Reconstruction}

The foundational step in the event reconstruction process is to separate and detect real signals from background noise in the raw waveforms. The signals expected in a wire readout TPC are pulse-like — called "hits" — and provide information about the time, location, and magnitude of the drift electron signals on the wires. The automated detection of hits is vital to the analysis of TPC signals, in general. The accurate calculation of hit parameters is important for nearly any physics analyses, including calorimetry. There are many approaches to hit reconstruction (or "hit finding"), the standard methods are included in LArSoft, and the non-standard algorithms described below were also written within the LArSoft framework for easy integration.

The same hit reconstruction algorithm can be used on both real data as well as simulated samples. Furthermore, because the user controls the simulation, the true ionisation hit parameters are known, and the performance of the simulation can be easily studied. Therefore, assuming accurate simulation of 35-ton data, the reconstruction performance results from the simulation can be extended to the interpretation of the real data reconstruction.

\subsubsection{Standard Hit Reconstruction}

By default in many Fermilab-based LAr TPCs, hit reconstruction is performed by the so-called "GausHitFinder" (GHF) [168]. The basis for this code is the hypothesis that any pulse can be 
modelled by a sum of Gaussian functions. The amplitudes and integrals of these Gaussians are well defined and fast to compute, making the algorithm desirable for fast and accurate hit reconstruction.

In this method, a pulse is found when the waveform rises above a predefined threshold ADC value. Once triggered, the algorithm searches for the endpoints of this pulse, to give a start and end point in time. Then, the pulse is fit to an increasing number of summed Gaussian functions, until the $\chi^{2} /$ N.D.F. falls below a predefined value.

Despite the widespread use and acceptance of this algorithm, for the 35-ton noise situation this method failed spectacularly due to the fixed hit finding threshold implemented for all wires. Figure 5.6b shows several features of this failure, including several wire signals with high hit multiplicity, implying that high random noise fluctuations were reconstructed as hits rather than being ignored as background. The reconstruction also bypassed some wire signals where hits should have existed, based on the existence of adjacent hits from a linear particle track. The low $\mathrm{S} / \mathrm{N}$ of the 35-ton and the variance of noise between channels and between events led to the dramatic increase in false hits created, reducing the purity of the hits collection. It also made further event reconstruction difficult due to the inability of the algorithms to distinguish between real hits from ionised charge and fake hits created from random noise fluctuations. Also, since for this analysis it was preferred to skip the deconvolution process in favour of retaining better hit charge calculations, the GHF was not useful because it was developed and optimised for use on deconvolved signals. Therefore, for this analysis, an alternate hit finding algorithm was developed.

\subsubsection{Robust Hit Reconstruction}

The "RobustHitFinder" (RHF) (see Figure 5.7) was developed specifically for the 35-ton experiment and the particular noise characteristics which made the standard hit finding techniques suboptimal. The RHF is based on three main premises:

- the expected event topology will always be a mostly straight particle track triggered by the external muon counters,

- the noise level is not constant across channels or events,

- and the hit parameters calculated on raw waveforms are less biased than if calculated on filtered and deconvolved waveforms.

The first premise specifically takes advantage of the unique 35-ton data source. Because the 35-ton was not in a beamline, the main source of particles was showers from high energy cosmic ray interactions in the upper atmosphere. The muon triggering system of 


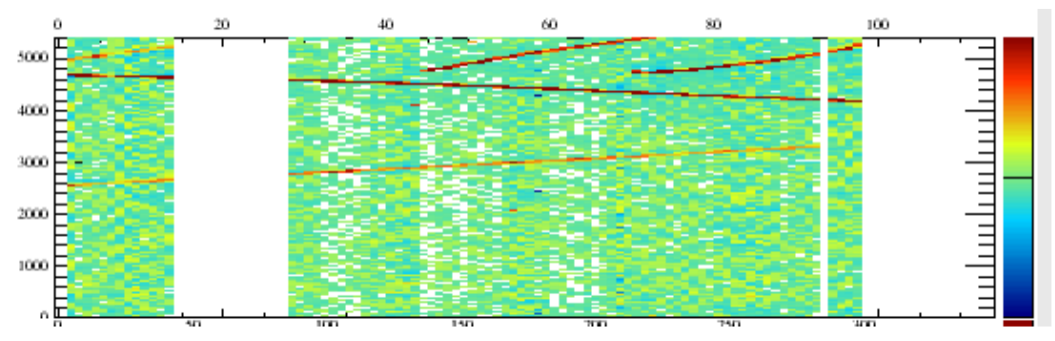

(a)

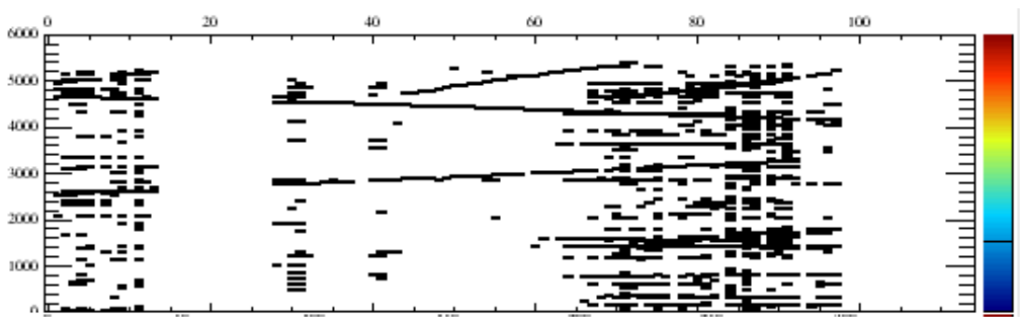

(b)

Fig. 5.6 Event displays showing time on the vertical, and wire number on the horizontal. (a) Signals on the wire are shown after deconvolution and frequency filtering. (b) Black dots represent reconstructed hits from the GHF.

the detector (Section 3.2.5) naturally only selected throughgoing muons which had mostly straight trajectories through the entire cryostat. The expected tracks of minimally ionising muons would be straight, with the occasional kink in the track due to multiple scattering, and possible track-bending due to space charge distortions of the drift electric field. Placing a prior expectation on the topology of the event helped especially to filter out interesting signals from background noise, even if the noise was particularly high.

The second premise above was addressed by using a different hit reconstruction thresholding scheme. While the GHF uses a fixed absolute amplitude threshold for all channels, the RHF uses a fixed standardised amplitude threshold. The ADC values in a waveform were standardised to the particular wire noise RMS value and baseline,

$$
\mathrm{ADC}_{\text {standard }}=\frac{\mathrm{ADC}-\text { Baseline }}{\mathrm{RMS}}
$$

and when the value raised above a predefined threshold (nominally, $2 \times \mathrm{RMS}$ ), a hit was recorded. This scheme has the benefit of more consistently dealing with high electronic noise which varied across the wires in the TPC, and for even lowering the threshold in the case where the wire noise was particularly low. Additionally, this accounted for differences in the absolute gains between wires, which could vary as much as 5-10\%. 


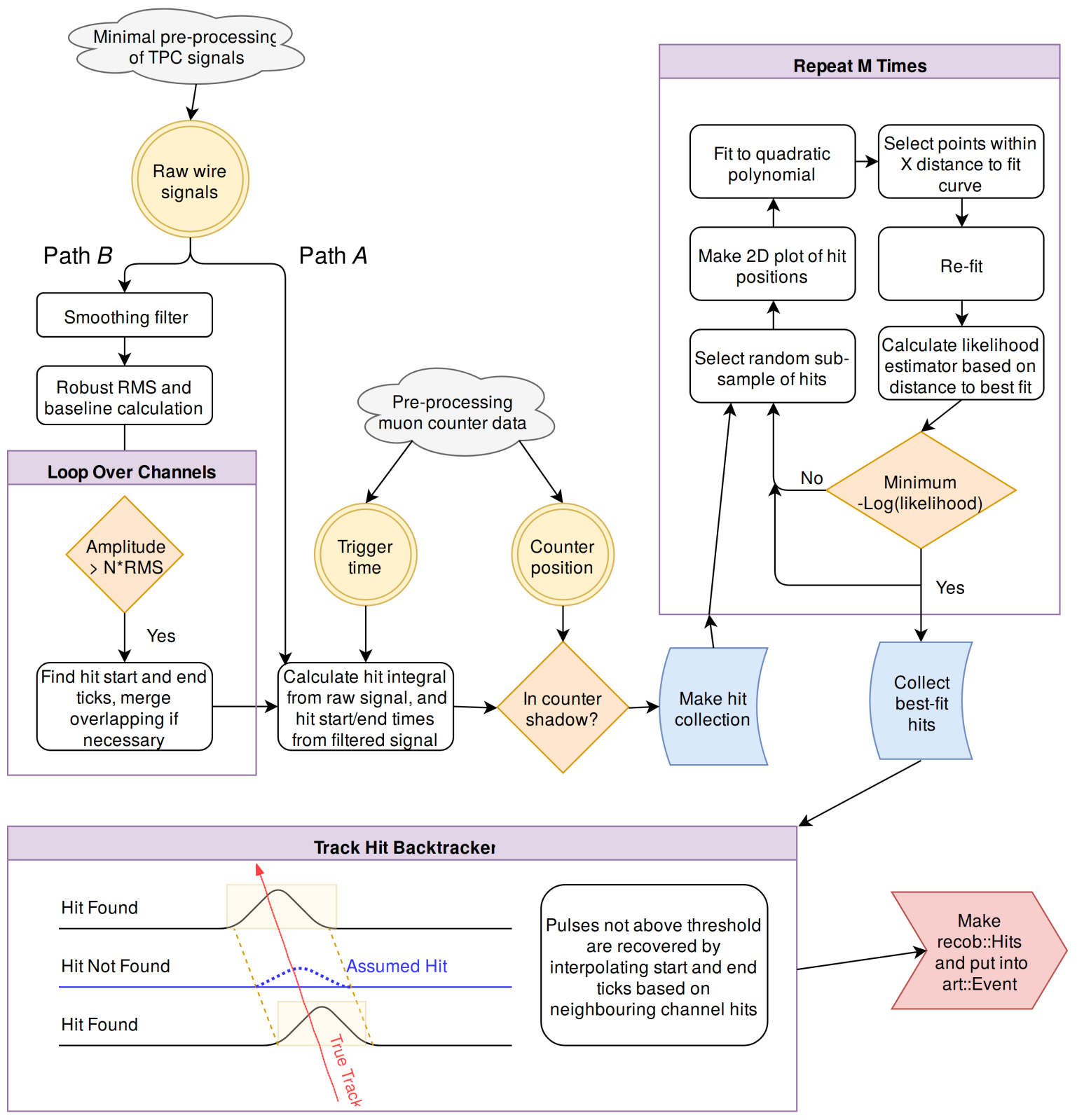

Fig. 5.7 Flow diagram of the RHF algorithm. 
The third premise addresses the non-linearities in reconstructed hit charge as seen in Figure 5.8a where the deconvolved pulse integrals, as measured by the GHF, did not linearly represent the true charge. The RHF, however, presented an excellent linearity between reconstructed pulse integral and true charge in Figure 5.8b, and therefore over the range of interesting hit charges, it was better to measure hit parameters from the unfiltered and un-deconvolved waveforms.

\subsubsection{Input Signals}

Collection wire signals input to the RHF were minimally preprocessed before applying the hit finding. Simply, if the channel was not in the bad channel list (Section 5.2.1), it was passed through the stuck bit mitigation (Section 5.2.3) and pedestal subtraction (Section 5.2.2) modules. No further noise removal or deconvolution was applied, to preserve the linearity of pulse integral with respect to input charge as much as possible. Stuck bit mitigation was retained because it is inherently a conservative process, where the five tick $(2.5 \mu \mathrm{s})$ limit on interpolation is shorter than the shaping time of the preamplifier. If the stuck bit mitigation was skipped, the integral or amplitude of any given pulse could have been skewed significantly since the stuck ADCs may have been as much as 64 ADCs $(23 \mathrm{mV})$ from the real value.

Induction wire signals were not used in the RHF for two reasons. First, the algorithm was only optimised to find unipolar pulses within a noisy signal. Use of induction wire signals would require field shape deconvolution in order to turn the bipolar pulses into unipolar pulses whose amplitudes are proportional to the number of induction electrons. Since this deconvolution process was not used, it was not possible to accurately calculate the hit size from the bipolar pulses. The second reason why induction wire signals were not used in this simulation and analysis is that the electronic noise on these channels was significantly greater than on the collection wire signals. This is simply because the induction plane wires were longer (since they wrapped around the APAs) and therefore had higher channel-to-channel capacitance.

\subsubsection{Hit Finding}

The hit finding algorithm proceeded along two parallel paths for every collection plane wire, copying the raw waveform to each path, $A$ and $B$ (see Figure 5.7). Path $A$ simply retained the input signal, without any other processing. Path $B$ began by performing noise filtering in the time domain. Here, a recursive single pole low pass filter [167] was used which has the form

$$
y_{n}=(1-a) x_{n}+a y_{n-1}
$$




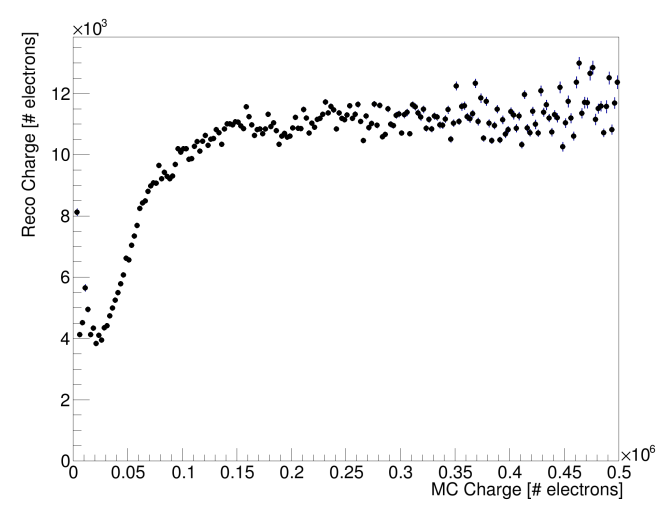

(a) Gauss Hit Finder.

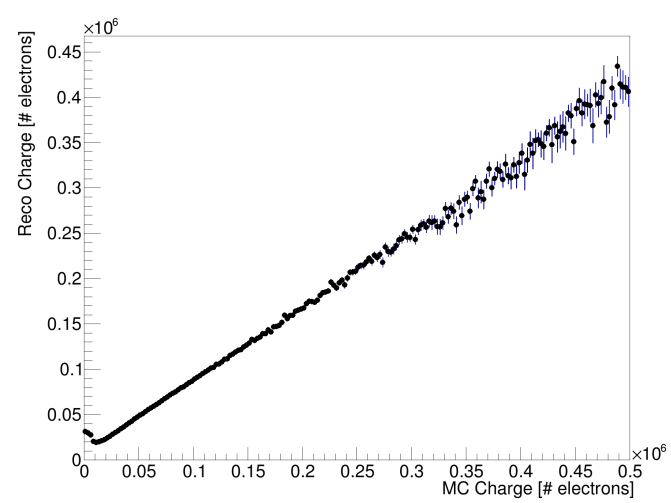

(b) Robust Hit Finder.

Fig. 5.8 Profile histograms of reconstructed hit integral vs. true hit charge for two algorithms.

where $y_{n}$ is the $n$ 'th sample of the output waveform, $x$ is the input waveform, and $a$ is a constant defined by $a=e^{-1 / d}$ where $d$ is the number of samples for the filter to decay to $36.8 \%$ of its original value. For the 35-ton, $d$ was set to 6 samples, giving a $-3 \mathrm{~dB}$ cutoff frequency of $53 \mathrm{kHz}$. This filter was applied twice to each waveform, once forward (in the direction of increasing TDC ticks), and once backward (decreasing TDC ticks) to reverse the positive phase introduced to the signal which is typical in a filtering application of this type in the time domain. This filter was designed to be conservative in the frequencies which are filtered since all signals were expected to have a pulse period much longer than $1 / 53 \mathrm{kHz}=18.87 \mu \mathrm{s}$ and should be retained. This filtering step was only implemented to make the finding of signals easier, rather than to reduce the impact of noise on the pulse integral and amplitude.

Before the standardisation of the ADC waveforms, the baseline and RMS of the filtered waveform were calculated. A windowed approach was taken to minimise the impact of signals on the RMS measurement. A window with 50 tick width was scanned across the waveform. For each window in the scan, the median ADC value and RMS were calculated and stored. The median of each measurement across all windows was calculated and set as the baseline and RMS of the channel. Standardisation of the filtered waveforms then occurred according to Equation 5.5.

A hit was found when the standardised ADC raised above 2.0. Given truly random noise, it was expected that $2.5 \%$ of the ADCs on the waveform would be above this threshold in the absence of real signal. This fraction of ADCs would, according to the hit finding procedure, get reconstructed as hits. Further steps were implemented to remove these extra fake hits (see Section 5.3.3).

Once a hit was triggered, the algorithm determined the start and end TDC ticks. A fixed hit width of 50 ticks before the hit trigger and 100 ticks following the hit trigger, or total 
$75 \mu$ s real time, was used. This width was expected to cover the possible true hit widths given the TPC drift velocity, the range of track angles with respect to the APA plane, and the electronics shaping time. This was implemented to reduce systematic errors caused by noise fluctuations. For instance, if a real pulse occurred at the same time as a positive fluctuation of the noise above the baseline, the pulse would artificially look larger and have a broader time base, and an automatic determination of the pulse leading and falling edge would be biased. By fixing the width, the hit parameters would be less sensitive to these types of biases, and, on average, the measured pulse integral would be accurate, assuming a correct baseline measurement. However, the variance of the measured hit parameters, compared with the true hit parameters, would be much more significant.

\subsubsection{Hit Parameter Calculation}

Once the begin and end tick values for each pulse were recorded for the filtered waveform in path $B$, these values were passed to the unfiltered waveform in path $A$, on which the hit parameters were calculated. Parameters calculated for each hit included:

- Peak Tick: Weighted mean tick between start and end of pulse. Weight function for tick $i$ is $w_{i}=\exp \left[\left(A_{i}-\sigma\right) / \sigma\right]$ where $A_{i}$ is the ADC value of tick $i$, and $\sigma$ is the waveform noise RMS.

- Hit Start Tick: Peak tick minus 50 ticks.

- Hit End Tick: Peak tick plus 100 ticks.

- Peak Time: Peak tick divided by the DAQ sample rate.

- Drift Time: Peak time minus muon counter trigger time.

- Hit Charge: $\sum_{\text {tick=start }}^{\text {end }}$ (waveform[tick] - baseline).

If there were two hits created on the same waveform where the first hit end tick was greater than the second hit start tick, the two hits were merged. At this point, the waveforms themselves were discarded from the reconstruction chain, and the reconstructed hit objects placed into a collection.

\subsubsection{Drift Distance}

Before further processing of the hit collection could be completed, information about the triggered event was gathered to calculate the drift distance. In particular, knowledge of the triggered muon scintillation counters' locations helped to define the region to search for valid hits. Trigger information was stored in a LArSoft data product, raw : : ExternalTrigger, 
which contained timing and physical hardware identification information. The timing information was used to define the event $t_{0}$, the time at which the particle interacted in the detector, to within one TDC sample time of $62.5 \mathrm{~ns}$. The coordinate of the ionisation along the drift direction was calculated taking into account the different electric fields in all drift regions, including between the induction and collection planes. The drift velocity between each pair of adjacent wire planes on each APA was assumed to be constant, an average drift velocity, to simplify the calculation. The drift coordinate $x$ at time $T=t_{\text {hit }}-t_{0}$ was calculated using the following equation

$$
x(T)= \begin{cases}\text { rejected, } & T<0 \\ v_{3}(T), & 0<T \leq \frac{l_{3}}{v_{3}} \\ l_{3}+v_{2}\left(T-\frac{l_{3}}{v_{3}}\right), & \frac{l_{3}}{v_{3}}<T \leq \frac{l_{3}}{v_{3}}+\frac{l_{2}}{v_{2}} \\ l_{3}+l_{2}+v_{1}\left(T-\frac{l_{3}}{v_{3}}-\frac{l_{2}}{v_{2}}\right), & \frac{l_{3}}{v_{3}}+\frac{l_{2}}{v_{2}}<T \leq \frac{l_{3}}{v_{3}}+\frac{l_{2}}{v_{2}}+\frac{l_{1}}{v_{1}} \\ l_{3}+l_{2}+l_{1}+v_{d}\left(T-\frac{l_{3}}{v_{3}}-\frac{l_{2}}{v_{2}}-\frac{l_{1}}{v_{1}}\right), & \frac{l_{3}}{v_{3}}+\frac{l_{2}}{v_{2}}+\frac{l_{1}}{v_{1}}<T \leq \frac{l_{3}}{v_{3}}+\frac{l_{2}}{v_{2}}+\frac{l_{1}}{v_{1}}+\frac{l_{d}}{v_{d}} \\ \text { rejected, } & T>\frac{l_{3}}{v_{3}}+\frac{l_{2}}{v_{2}}+\frac{l_{1}}{v_{1}}+\frac{l_{d}}{v_{d}}\end{cases}
$$

where variables $v$ and $l$ represent the drift velocity and the full distance between planes, respectively. Subscripts $d, 1,2$, and 3 represent the gaps between the cathode and grid, the grid and first induction plane, first induction plane and second induction plane, and the second induction plane and the collection plane, respectively. The slopes of the line segments in Figure 5.9 show the differences in velocity between the wire planes. If a hit had reconstructed time outside of the range defined by the TPC boundaries, it was considered unphysical and most likely due to a background event or noise, and was rejected.

Due to uncertainties in the drift velocity and cold dimensions of the TPC and wire planes, hits with reconstructed positions very near the boundaries of the TPC were usually treated with less confidence than ionisation hits from the middle of the TPC.

\subsubsection{Fiducialisation of the Event}

In addition to timing information from the triggered muon counter information, the physical location of the muon counters in the detector hall, with respect to the TPC, helped to fiducialise the search for "true" triggered hits and tracks, and ignore background cosmic events. The two-dimensional hit position was used along with the known positions and sizes of the counters to define a "counter shadow", a region within which the particle track must be contained. If a hit was reconstructed within a distance of $25 \mathrm{~cm}$ (slightly more than the half width of a muon counter) to a line which connects the centres of the two triggered muon 


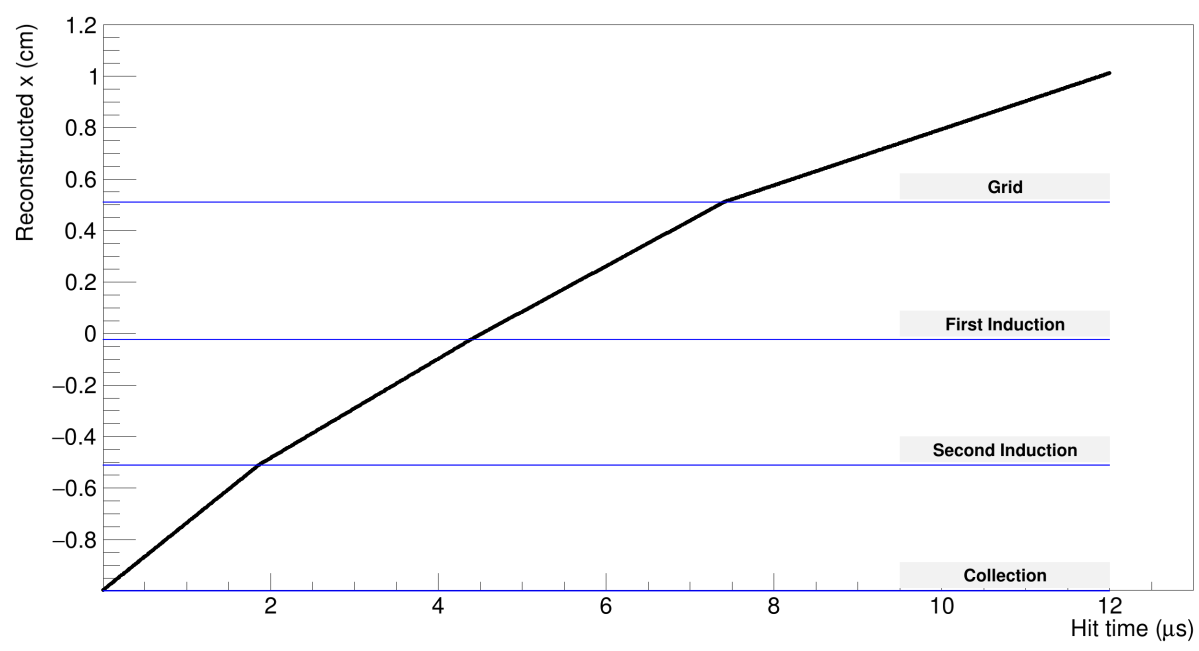

Fig. 5.9 Reconstructed drift coordinate versus hit time. The slope of the line represents the drift velocity in the particular region of the APA frame.

counters, it was accepted, while all other hits were assumed to be fake noise hits and removed from the hits collection.

\subsubsection{Track Finding}

The next step in the purification of the hits collection was the application of a robust fitting algorithm, Maximum Likelihood Estimator Sample Consensus (MLESAC) [169], adapted from the field of computer vision. The algorithm, which generalises the Random Sample Consensus estimator (RANSAC) [170], uses an iterative process to randomly sample from a set of 2D points (hits), compute a best-fit model, and maximise the log likelihood estimator (or minimise the negative log likelihood estimator). It is robust in the sense that outliers (below the threshold for model inclusion) are weighted much less than inliers (above the threshold for model inclusion). In extreme cases, outliers have zero weight and do not contribute to the likelihood function.

The algorithm, as implemented in the hit reconstruction for the 35-ton, began by mapping the hits collection onto a 2D projection of the TPC: drift coordinate $(x)$ vs. wire coordinate $(z)$. In this view, tracks could be seen as a linear or slightly curved grouping of hits extending from one wall of the TPC to another with background particle tracks and fake noise hits scattered randomly throughout, as in Figure 5.10. Confirmation of the detection of a triggered muon is possible by observing the track to be within the counter shadow region of acceptance for the event given the triggered muon counters. 


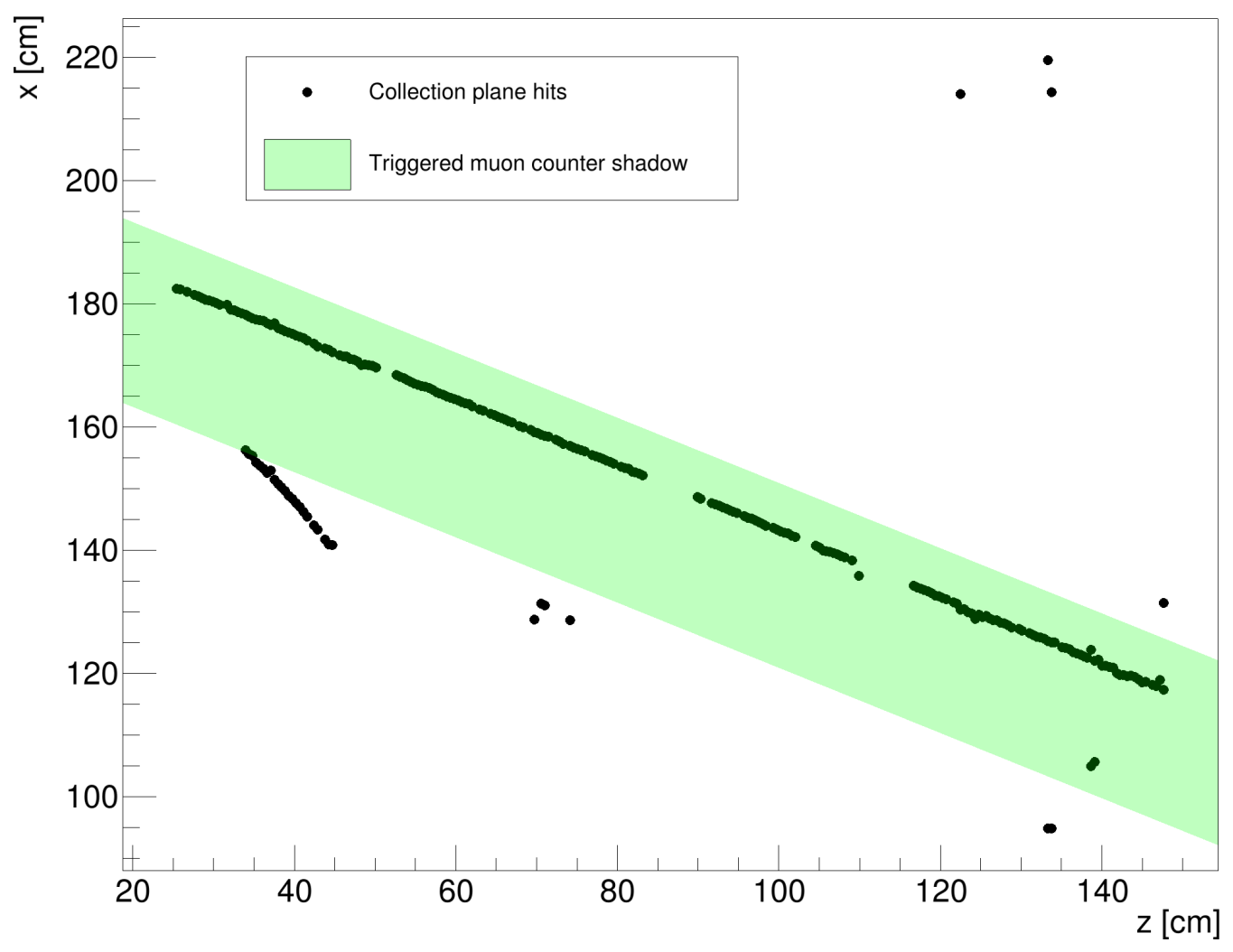

Fig. 5.10 Example event with 246 hits in the TPC, plotted at their detector $x$ and $z$ coordinates. Also shown in green is the region of acceptance for this event given the triggered muon counters. 
The algorithm proceeds iteratively to robustly determine a model which fits the data. On each iteration, three hits are randomly sampled from the total event hits population. This initial sample is represented by the red points in Figures 5.11 and 5.12. These points are fit to a second degree polynomial where the highest order term was limited to only very small possible values to allow track bending due to space charge distortions and multiple scattering of the muon in the detector. Following the fit to the sample points, the algorithm searches the remaining population points for any additional hits which occur within a predetermined distance from the initial best-fit curve (inliers, blue points in Figures 5.11 and 5.12). For the 35-ton, this threshold distance was set to be $3 \mathrm{~cm}$, which was chosen to take into account the temporal width range of the hits when converted to distance using the drift velocity and the range of track angles, while removing some of the delta ray tracks emanating from the original muon track. This threshold is indicated by the thin black lines on either side of the best fit red line in Figures 5.11 and 5.12. A newly expanded sample was created with the inliers (blue points), and refit to the same model (red line). Based on the high $(>1)$ sum of squared residuals per degree of freedom (S.S.R./N.D.F.), it is clear that the initial starting sample in Figure 5.11 does not represent a good reconstruction of the true muon track. However, a subsequent iteration of the algorithm (Figure 5.12) randomly selected a different starting sample, which led to a good model fit. This good fit has a small S.S.R./N.D.F. and is parallel to the shadow of acceptance region connecting the triggered muon counters, implying the correct muon track fit. The number of iterations for an event with $n$ reconstructed hits is $n \times 20$, which ensures a very high probability that at least one iteration of the random sampling process selects three real muon ionisation hits, essentially guaranteeing a good fit.

A maximum likelihood approach is used to score each best-fit model on each iteration of the algorithm for the given event. On each iteration, the score is the negative log likelihood function which combines the inlier and outlier probabilities of the $n$ data points:

$$
-L=-\sum_{i}^{n} \log \left[p_{i}^{\text {inlier }}+p^{\text {outlier }}\right]
$$

The likelihood of a datum to exist given that it is an inlier, $p_{i}^{\text {inlier }}$, is given by

$$
p_{i}^{\text {inlier }}=\gamma\left(\frac{1}{\sqrt{2 \pi} \sigma}\right) e^{-d_{i}^{2} /\left(2 \sigma^{2}\right)},
$$

which is based on a Gaussian assumption of errors on the true location of the hit in 2D space where $d_{i}$ is the distance of point $i$ to the best fit curve (the error on the coordinate), $\sigma$ is the standard deviation of the error on each coordinate, and $\gamma$ is a mixing parameter determined in a later step. To account for the presence of outliers randomly scattered across the sample 


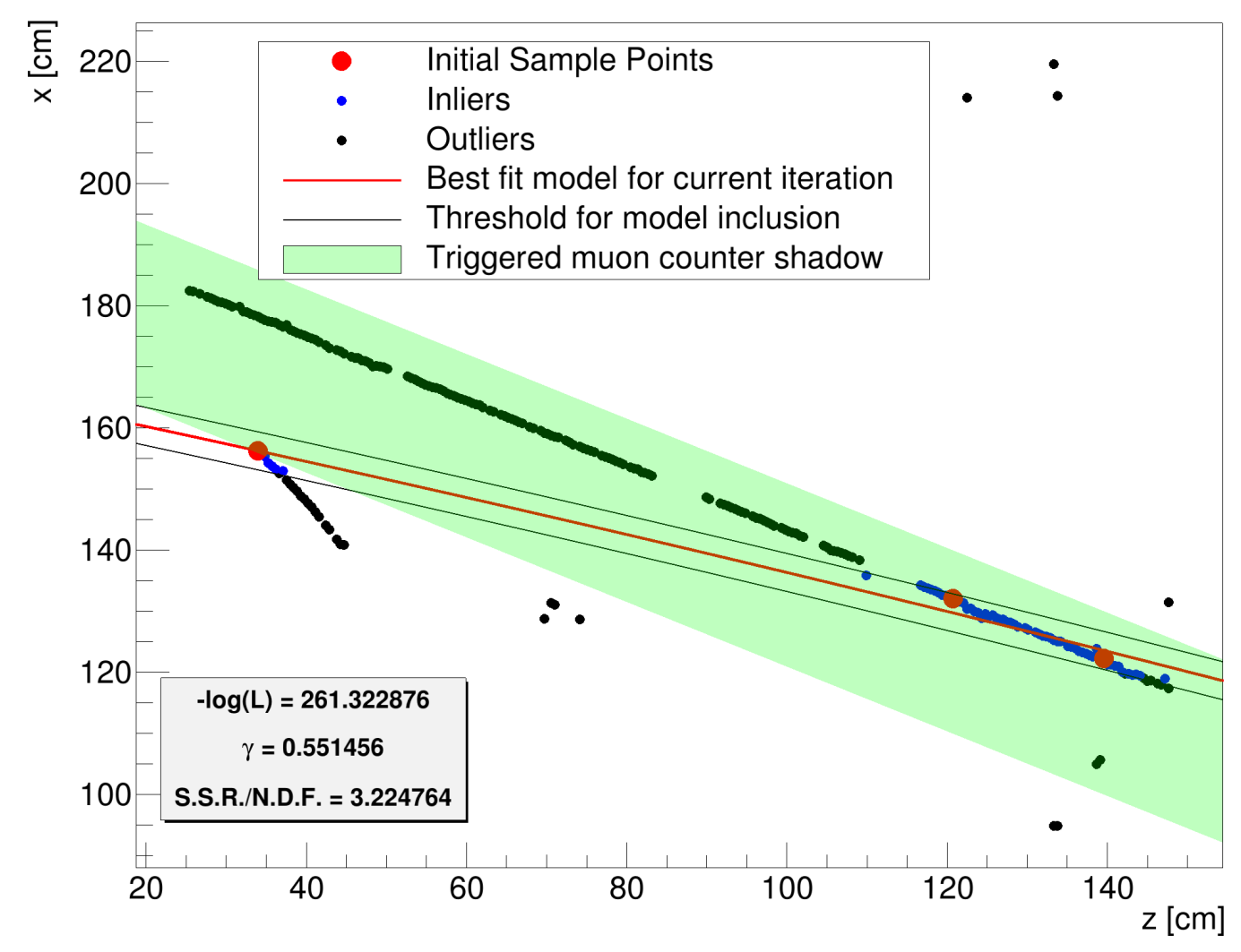

Fig. 5.11 Example iteration of the MLESAC algorithm applied to the event in Figure 5.10. Given the initial starting randomly sampled points (red points), the best-fit model (red line) does not represent a good fit to the actual physical event, indicated by a high sum of squared residuals (S.S.R.) per degree of freedom (N.D.F.). 


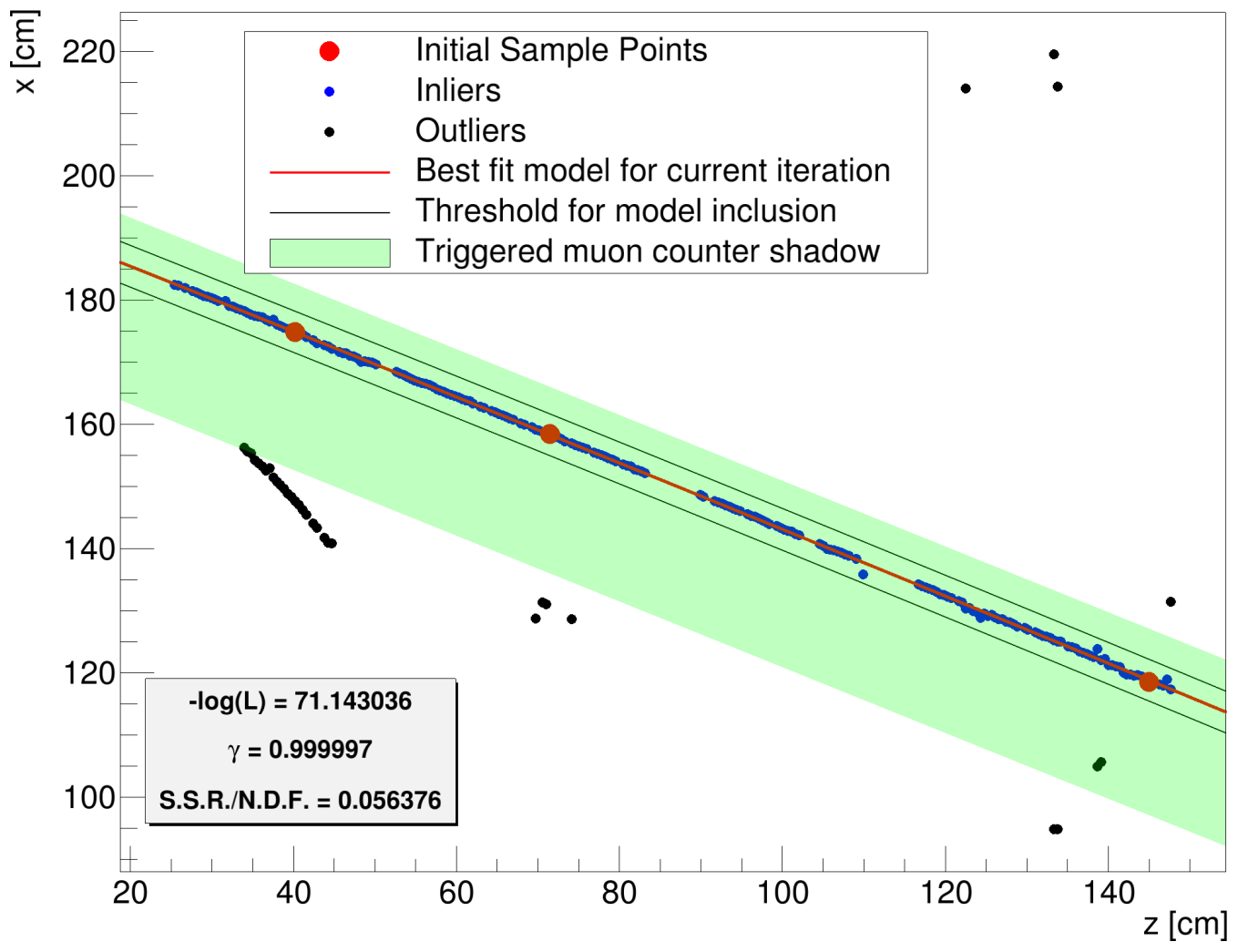

Fig. 5.12 Example iteration of the MLESAC algorithm applied to the event in Figure 5.10. Given the initial starting randomly sampled points (red points), the best-fit model (red line) represents a good fit, indicated by a low sum of squared residuals (S.S.R.) per degree of freedom (N.D.F.). 
space, the likelihood of a datum given that it is an outlier, $p^{\text {outlier }}$, is constant for all hits within the entire TPC,

$$
p^{\text {outlier }}=(1-\gamma) \frac{1}{v}
$$

where $v$ is the size of the search window. The search window is defined as the length of the hits population bounding box diagonal, or in other words the length of the TPC diagonal in $x-z$ space, $\sqrt{\left(x_{\max }-x_{\min }\right)^{2}+\left(z_{\max }-z_{\min }\right)^{2}}$.

The final remaining factor in calculating the likelihood function for the algorithm iteration is $\gamma$, which is interpreted as an estimate of the proportion of inliers in the data set. While the iterative model-fitting step above designates each point to be an inlier or an outlier based on a simple threshold of the absolute distance of each point to the best-fit line, this assumed proportion of inliers does not necessarily maximise the likelihood function. Adjusting $\gamma$ effectively changes the threshold for model inclusion by reweighting the Gaussian probability for inliers and the constant background noise probability for outliers. So, a point at a given distance from the model may have a higher probability of being an inlier or an outlier, depending on the value of $\gamma$ for which the likelihood function is maximised. This value of $\gamma$ depends ultimately on the distribution of data points and their uncertainties.

In the MLESAC process, the $\gamma$ factor is an unknown, and is determined using the method of expectation maximisation [171]. The process requires the introduction of a set of unknown variables, $\eta_{i}$, where $\eta_{i}=1$ if point $i$ is an inlier, and $\eta_{i}=0$ if point $i$ is an outlier. Denote the expected value of $\eta_{i}$ as $z_{i}$. The calculation is as follows:

1. Generate an initial guess, $\gamma=0.5$.

2. Estimate the expected value of $\eta_{i}$ for each point using the current estimate of $\gamma$, and the probabilities in Equations 5.9 and 5.10,

$$
z_{i}=\frac{p_{i}^{\text {inlier }}}{p_{i}^{\text {inlier }}+p^{\text {outlier }}} .
$$

3. Make a new estimate of $\gamma$ using the current expected values of $\eta_{i}$,

$$
\gamma_{\text {new }}=\frac{1}{n} \sum_{i}^{n} z_{i}
$$

4. Repeat steps 2 and 3 until convergence, usually less than 5 iterations.

When the $\gamma$ factor is deduced, the log likelihood function is calculated for the given iteration.

Once each of the random subsamples of data are scored using the negative log likelihood function in Equation 5.8, the minimum $-L$ across all random samples denotes the best fit. 
The hits which comprise the best fit sample (inliers) are taken as the "true" hits and the outliers are ignored as "fake" noise hits.

While the MLESAC implementation in the RHF was computationally expensive (more than $90 \%$ of total time to run, per event), it was effective in selecting real from fake hits. It was, therefore, possible to lower the hit finding threshold (i.e. allow more "fake" noise hits in the original hits collection) and still select the true hits on the track. The final threshold of $2 \times R M S$ was chosen by maximising the hit finding efficiency. Compared with the default threshold for experiments which use the GHF, 3.6 times the ideal noise RMS, this method gave a substantial improvement on hit finding in the presence of high noise. Details of hitand track-finding efficiencies and purities of the RHF are discussed in Chapter 6.

\subsubsection{Track Hit Backtracker}

While the MLESAC implementation had the effect of increasing the purity of the hits collection, the Track Hit Backtracker (THB) had the effect of improving the efficiency of hit finding. The major downside with using a variable hit threshold was that it still may be too high to find the smallest hits. For a minimally ionising particle, the energy loss per unit length has a Landau probability distribution function [172]. Since energy loss in LAr is proportional to ionisation charge, the hit charge also follows a Landau distribution. If the hit finding threshold were too high, it would be possible for the hits in the lower tail of the Landau not to be identified by the hit finder, whereas the large charge hits would always be found. Depending on the threshold, some fraction of hits along a track might be found, which is directly related to the hit finding efficiency. Short of lowering the hit finding threshold (which, again, would reduce the purity of the hits collection), it was still possible to recover the lost low charge hits.

The process of recovering the small charge hits assumed that the muon tracks which were found in the MLESAC step were not discontinuous. That is, it was expected that a charged particle which traversed the detector (along $z$ ) and ionised charge along the track deposited charge on every collection plane wire, regardless of the noise level on that wire. For example, if there were seven consecutive wires, the first three with MLESAC-determined true hits, one wire without any hits found, and then three more wires with true hits, it was assumed that the wire with no hits did actually have a charge deposit, but the signal it generated was too small to be triggered by the hit finder. Given this assumption, the THB attempted to recover these hits by interpolating the location (start and end ticks) of the missing hits based on adjacent "true" hits (also called "found" hits). The recovered hits from this process were denoted as "assumed" hits. 
To be conservative in these assumptions, the THB never extrapolated. That is, to be "assumed", a channel without hits must have had adjacent channels with hits on both sides in $z$. Therefore, even with this method, it was not possible to recover all of the hits lost within the noise if the wire without a hit was an edge wire on the APA.

The integrals and peak times of the "assumed" hits were calculated in the same way as the "true" hits to reduce further systematic errors between the two types of hits.

\subsection{Event Reconstruction}

In normal reconstruction, the hits collection (stored in LArSoft as recob: : Hit objects) would be passed to further modules which perform more and more complex tasks to fully reconstruct the event. These steps include:

- Clustering. Grouping hits into 2D objects which represent the ionisation of a single physical entity,

- Tracking. Grouping 2D clusters into 3D tracks if the grouping is track-like (i.e. linear),

- Showering. Grouping 2D clusters into 3D showers if the grouping resembles a shower rather than a track,

- T0. Using optical or trigger information to determine event interaction time

- Particle Identification. Event level reconstruction where tracks and showers (and even the absence of tracks and showers in the case of uncharged particles) are used to identify and label the particles which caused the event in the detector,

- Calorimetry. Analysis phase reconstruction step where $d Q / d x$ and prior calibrations are used to determine track $d E / d x$.

The electron lifetime analysis required low level hit reconstruction only, as it depended only on the drift distance of the ionisation. So for this analysis, all of these additional steps in the reconstruction chain were unnecessary.

\subsection{Summary}

The detailed simulation of the 35-ton detector and electronics was performed to most accurately reflect the particular conditions of the 35-ton Phase II run. The algorithm to reconstruct hits in the detector was primarily motivated by the specific noise characteristics of the 35-ton, in light of the unsuitability of the standard LArSoft reconstruction algorithms to provide 
efficient and accurate hit parameter calculation. Both the simulation and reconstruction described in this chapter were performed with the goal of reducing the systematic errors and uncertainties in the argon electron lifetime measurement presented in the next chapter.

The reconstruction algorithms developed for the 35-ton data are, in principle, applicable to any LAr TPC, especially those with high electronic noise which degrades the data quality. Nevertheless, several possible improvements can be made to the algorithms which may make the reconstruction more generalisable. For example, the algorithm is currently only able to detect long straight particle tracks. It would be significantly improved with the ability to reconstruct shorter particle tracks (e.g. electrons) or showers. The addition of this, and other, functionality is the subject of further study. 


\section{Chapter 6}

\section{Analysis of 35-ton Electron Lifetime}

One of the fundamental detector characterisation studies for liquid argon (LAr) time projection chambers (TPC) is the analysis of the argon electron lifetime. The achieved lifetime has direct consequences on the overall physics capability of the detector, and its measurement is needed to calibrate the particle energy loss in argon. While the study is simple to complete, confounding factors such as excessive electronic noise dramatically complicate the analysis. Nevertheless, if the lifetime can still be measured under challenging circumstances, the operational requirements of the detector and data acquisition system might be relaxed.

As part of the 35-ton Prototype phase II experiment (see Chapter 3), the electron lifetime of the LAr was measured in two ways: with dedicated purity monitors, and using TPC data. This chapter presents the measurement of the electron lifetime of the 35-ton using TPC data. Also, the simulated samples produced according to the steps in the previous chapter are analysed to determine the performance of the reconstruction algorithms. The simulation is then used to better understand the systematic uncertainties in the data lifetime measurement.

\subsection{Electron Lifetime Analysis}

Free electrons in the LAr attach to electronegative impurities (such as oxygen and water), reducing their drift velocity, so they no longer contribute to hits, thereby reducing the total charge collected at the anode. As described in Chapter 2, the electron lifetime, $\tau$, is defined by the exponential decay of the collected charge at the anode, $Q_{\mathrm{c}}$, with drift time, $t$,

$$
Q_{\mathrm{c}}=Q_{0} e^{-t / \tau}
$$

where $Q_{0}$ is the charge liberated in the ionisation after recombination. For example, a reduction of charge collected of $Q_{\mathrm{c}} / Q_{0} \approx 51 \%$ is expected for a drift of $2.2 \mathrm{~m}$, an electric 
field of $250 \mathrm{~V} / \mathrm{cm}$, and an electron lifetime of $3 \mathrm{~ms}$. $\tau$ is related to the concentration of electronegative impurities in the argon by the empirical relation

$$
\tau=\left(\sum_{i} k_{i} n_{i}\right)^{-1}
$$

where $n_{i}$ are the number concentrations of the $i$ impurity species, and $k_{i}$ are proportionality factors which describe the relative strengths of attenuation affinity by the different species. Given a typical value for $k$ (e.g. $k_{O_{2}}$ in Section 2.2.3), the concentration of electronegative impurities for a lifetime of $3 \mathrm{~ms}$ is $O(\mathrm{ppt})$. Measuring $\tau$ is important in quantifying and correcting for the overall effect of impurities inside the TPC on the drifting ionisation charge. Therefore, measuring the electron lifetime with a high accuracy is a required calibration task for the next generation of massive liquid argon TPCs.

Complementary to the dedicated purity monitors, the electron lifetime of the liquid argon was studied using cosmic ray muon tracks in the TPC. Several experiments have successfully measured the electron lifetime using methods similar to the one described here $[50,114,118,128,142,173]$.

\subsubsection{Method}

Successfully reconstructed hits from the Robust Hit Finder (RHF) (Section 5.3.2) were used, along with the parameters of the track created by the track hit backtracker (THB) (Section 5.3.3), and the triggered muon counters to define the hit charge per unit length of track, $d Q / d x$. In discrete form (i.e. hit-by-hit), $d Q / d x$ becomes $Q / \gamma$ where $Q$ is the reconstructed hit integral. Other methods for determining the lifetime instead use the hit amplitude, however, this metric is less robust as it is more sensitive to fluctuations in the waveform baseline. To correct for differences in track angle in relation to the collection plane wires, $\gamma$ is defined as

$$
\gamma=\frac{\delta}{\cos \varphi \cos \theta}
$$

where $\delta$ is the wire spacing, $\theta$ is the angle between the track and the wire plane, and $\varphi$ is the angle between the track projection in the wire plane and the vector perpendicular to the wire, in the plane of the wire, as illustrated in Figure 6.1.

The reconstructed hit $d Q / d x$ values throughout the detector were aggregated over all events in the given data sample, then divided into bins of similar time over the full drift window which depends on the peak time of the hit with respect to the trigger time. In this analysis, the total drift distance, $220 \mathrm{~cm}$, was divided into 22 equal bins of $10 \mathrm{~cm}$, 


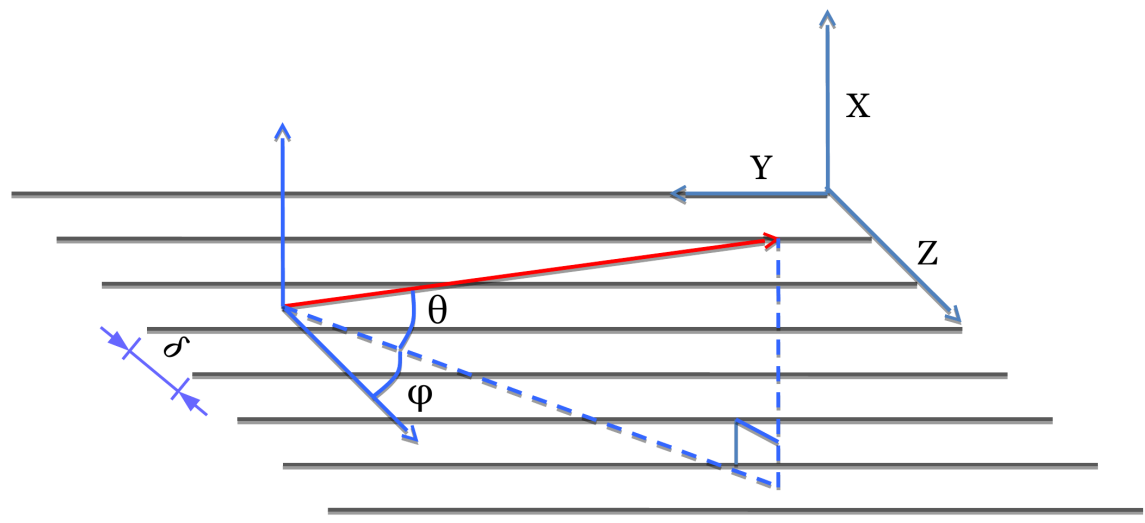

Fig. 6.1 Illustration of the variables which define $\gamma$ (Equation 6.3).

which correspond to $91.45 \mu$ s of drift time given the nominal electric field during the run of $250 \mathrm{~V} / \mathrm{cm}$, and the calculated drift velocity of $0.109 \mathrm{~cm} / \mu \mathrm{s}$. Distributions of $d Q / d x$ in each of these bins were fit to a Landau (muon energy loss due to ionisation) convolved with a Gaussian (detector resolution) to extract the most probable value (MPV) of the Landau.

The MPVs extracted from the fits in each of the bins was then plotted against the central value of drift time in that bin. The extracted MPVs were fit to Equation 6.1, where the electron lifetime was retrieved, along with fitting errors.

\subsubsection{Landau Gaussian Convolution Fits}

The convolution and fitting processes use the RooFit [174] toolkit, which is built into the ROOT framework [145]. First, the model was built using the RooFit class structure. An instance of RooLandau was created with two free parameters, $\mathrm{ml}$ for the Landau MPV, and sl for the Landau width. The RooLandau inherits from RooAbsPdf, a generic probability density function (PDF) class which can be fit to a data set. For the detector resolution, an instance of RooGaussian was created with one free parameter, sg for the Gaussian sigma or width, and one fixed parameter, $\mathrm{mg}$, with a value of 0 for the mean value of the function. The FFT was used to convolve the two PDFs in the RooFFTConvPdf class, which also inherits from RooAbsPdf. The method of convolution is simple and computationally efficient, aided by RooFit's optimised numerical integral caching process where the results of each integral were stored in memory for possible use in other iterations of the minimisation process.

The Landau Gauss Convolution PDF (shortened to "Landau $\otimes$ Gauss", or "L $\otimes$ G") was fit to each of the data $d Q / d x$ bins using the RooAbsPdf: : fitTo method with parameters to enable various options in the fitting process. First, the fit range was limited to $d Q / d x$ values which exclude the tails of the distribution. In particular, the low end of the fit was defined to 
be the first bin above $30 \%$ of the maximum histogram amplitude, and the high end at the last bin above $50 \%$ of the maximum amplitude. This range was chosen to include the rising edge of the PDF, which is important for fit convergence, and the peak of the distribution to determine the Landau MPV. Also, the range excludes hits with tiny charge, which have very low efficiency and charge reconstruction accuracy, as well as excluding the influence of delta electron ionisation, which has a most probable charge of roughly twice that of the Landau MPV from primary minimally ionising muons.

The fitting process uses the back end algorithms provided by Minuit2 (a $\mathrm{C}++$ implementation of the MINUIT package [175] which is built into ROOT), to perform the minimisation, and the MINOS error analysis package within Minuit2 to calculate the error matrix. These methods correctly calculate and propagate the errors in the data histogram, which are initially calculated by RooFit using the Poisson model for counting statistics. Therefore, the errors in fit parameters are expected to portray the statistical errors in the measurement accurately.

The $L \otimes G$ PDF was reparameterized in two ways to increase the stability of the convolution fits. First, the absolute values of the parameters were scaled to be within a reasonable range, from 0 to $O(10)$. For the parameters, this meant fitting the distributions with respect to $\mathrm{ml} / 1000, \mathrm{sl} / 100$, and sg/100 when using units of $\mathrm{ADC} / \mathrm{cm}$.

The other reparameterisation of the $\mathrm{L} \otimes \mathrm{G}$ fitting procedure involved the Landau distribution directly. As implemented by ROOT, the location parameter of the Landau function, $\mathrm{ml}$, is most closely related to the mean of the distribution (though the true mean of the Landau distribution is undefined as the area under the curve is infinite). However, for describing particle energy loss in a medium, the most probable value of the Landau is a more stable and useful parameter. According to the FORTRAN implementation of the Landau function in CERNLIB [176], the relationship between the location parameter and the MPV is:

$$
\text { MPV }=\text { Location }-0.22278298 \times \text { Width } .
$$

By reparameterising the Landau location parameter in terms of the width parameter, the true MPV could be extracted from the fits.

As a result of this reparameterisation, the statistical uncertainty of the MPV was propagated using the covariance between the location and width parameters:

$$
\sigma_{\mathrm{MPV}}=\sqrt{\sigma_{\text {Location }}^{2}+\left(0.22278298 \sigma_{\mathrm{Width}}\right)^{2}-2(0.22278298) \sigma_{\text {Location/Width }}}
$$

where $\sigma_{A}$ is the standard deviation of parameter $A$, and $\sigma_{A / B}$ is the covariance of parameters $A$ and $B$. 


\subsubsection{5-ton Data Set}

The dataset used in this analysis spanned five consecutive days of the Phase II run of the 35-ton, triggered on East-West crossing muons when the detector conditions were good. That is, when

- the electric field was stably maintained at $250 \mathrm{~V} / \mathrm{cm}$,

- the electron lifetime was greater than $2 \mathrm{~ms}$, as measured by the dedicated Purity Monitors (PrM),

- and the readout electronics were not in a high noise state.

Of the full data run, there were 20,395 events recorded during which these conditions were met. Various cuts (summarised in Table 6.1) were made to the data sample to account for non-ideal reconstruction and to select the most interesting events, leaving 17,490 analysable events.

For this data sample, only East-West muon triggers were used to accept tracks at small angles from the anode plane assemblies (APA). This reduced the effective track length acting on each wire signal, and in effect, reduced the average charge on each wire. These types of wire signals have a much better-modelled field response because of the short interaction time of each charge packet with each wire signal. Alternatively, if tracks with large angles were analysed, the effective track pitch length would be very long, indicating a significant absolute number of electrons would interact with the wires, over an extended period. Signals from these kinds of events are poorly modelled and highly sensitive to noise fluctuations.

The largest cut on the data sample was from poorly fitted tracks from the MLESAC step (Section 5.3.3). This was deliberately harsh to restrict the sample to only the tracks which were reconstructed within the fiducial volume of the detector, consistent with the triggered muon counter locations, and the sum of squared residuals per degree of freedom less than 3. The next cut removed channels which were located on the edge of each APA frame, as described in Section 5.2.1. Lastly, calculated hit $d Q / d x$ values outside of the range

Table 6.1 Number of data acquisition runs, triggered events, and both types of hits from the full initial data sample, following each subsequent data quality/acceptance cut.

\begin{tabular}{|l|r|r|r|r|}
\hline Cut & Runs & Events & "Found" Hits & "Assumed" Hits \\
\hline Initial Sample & 511 & 20395 & 3404957 & 1229788 \\
Successful Track Fits & 511 & 17490 & 2931704 & 1041427 \\
Non-Edge Channels & 511 & 17490 & 2907344 & 1041427 \\
$d Q / d x$ Within Range & 511 & 17490 & 2560360 & 812064 \\
\hline
\end{tabular}


$0 \leq d Q / d x \leq 6000 \mathrm{ADC} / \mathrm{cm}$ were truncated from each bin histogram, and excluded from the fits. Hits with integrals outside this region were located in the extreme low and high tails of the Landau, so were not interesting for this analysis.

The remaining distribution of hit $d Q / d x$ values (combined from all drift times) separated between "found" and "assumed" hits is shown in Figure 6.2. The total number of hits in each drift time bin, as well as the fraction of all hits which are "assumed" from the process in Section 5.3.4 are shown in Figure 6.3. The increase in "assumed" hit fraction with drift distance is consistent with the hypothesis that the reduction in hit charge resulting from attenuation by impurities causes the signals to reduce in size below the hit finding threshold.

\subsubsection{Preliminary Exponential Decay Lifetime}

Four of the 22 drift time bins of $d Q / d x$ are shown in Figure 6.4, along with the corresponding Landau $\otimes$ Gauss best-fit curve. In each bin, the fitting procedure returned a successful completion code, and accurate error matrix according to the MINOS algorithm. A plot of the fit $\chi^{2}$ /N.D.F. is shown in Figure 6.5, showing the success of the fits.

By plotting the extracted Landau MPV versus drift time in $\mu$ s (Figure 6.6), the attenuation of charge over the full drift is evident. At short drift times, the most probable $d Q / d x$ exceeded $2400 \mathrm{ADC} / \mathrm{cm}$ and decreased to around $1600 \mathrm{ADC} / \mathrm{cm}$ for long drifts. Within reasonable uncertainties on the observed bin MPV, the attenuation is almost exponential, with a slight upward bias on MPVs from long drift times. A fiducial cut of 5\% to 50\% of the full drift time was imposed to reduce bias due to incorrectly determined MPV for longer drift hits, and possible biases in the first bin nearest to the anode (further explanation is given in Section 6.4.5). An exponential fit to the values within the fiducial region gives a raw decay lifetime of $\tau_{\text {raw }}=4.24 \pm 0.10$ (stat.) ms. The systematic uncertainties on this measurement are discussed in detail in Section 6.5.1.

When comparing the lifetime measured by the TPC with the lifetime measured by the dedicated PrM system (Section 3.4.3), there is a discrepancy. The value reported by the PrM2 for the same data set $(2.8 \pm 0.1$ (stat.) \pm 0.5 (syst.) $\mathrm{ms}$ ) is consistent with the lifetime measurement presented here and its statistical uncertainty to within $2.4 \sigma$. The discrepancy is not altogether unreasonable, but still a possible sign of a significant error. Further insights into this discrepancy and the implied bias on the measurement of electron lifetime are gained through analysing the reconstruction performance of the algorithms. 


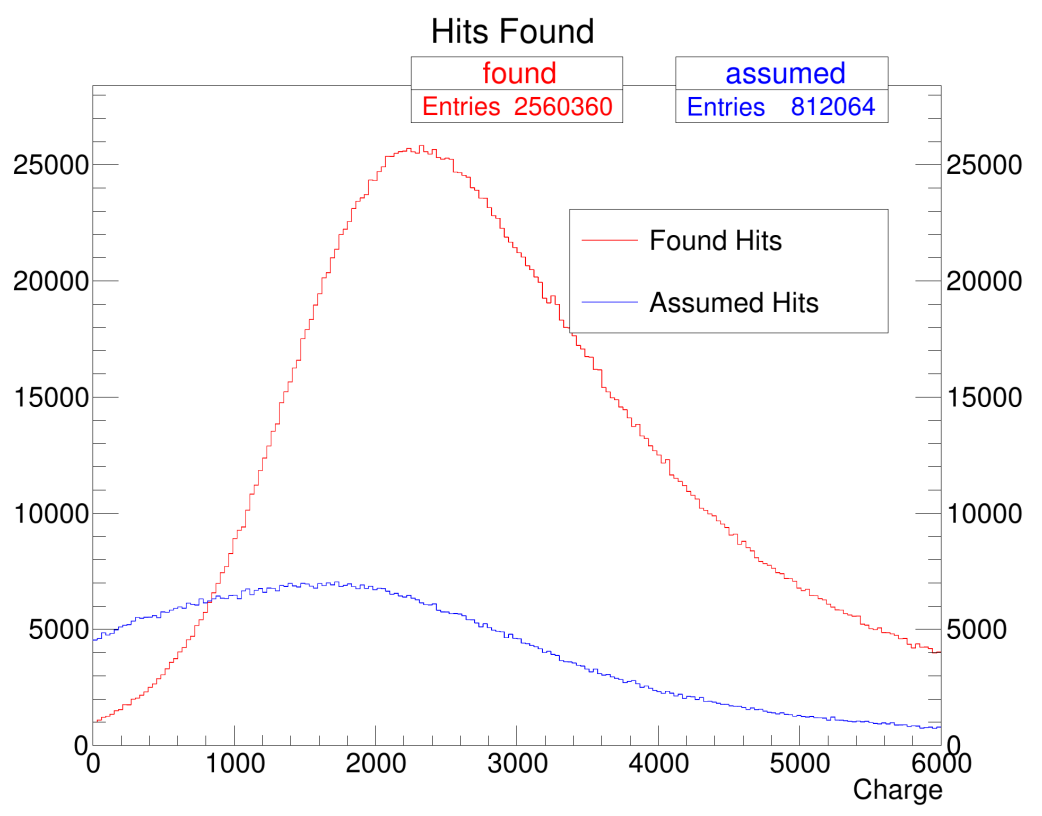

Fig. 6.2 Distributions of hit $d Q / d x$ for "found" and "assumed" hits across all drift times.

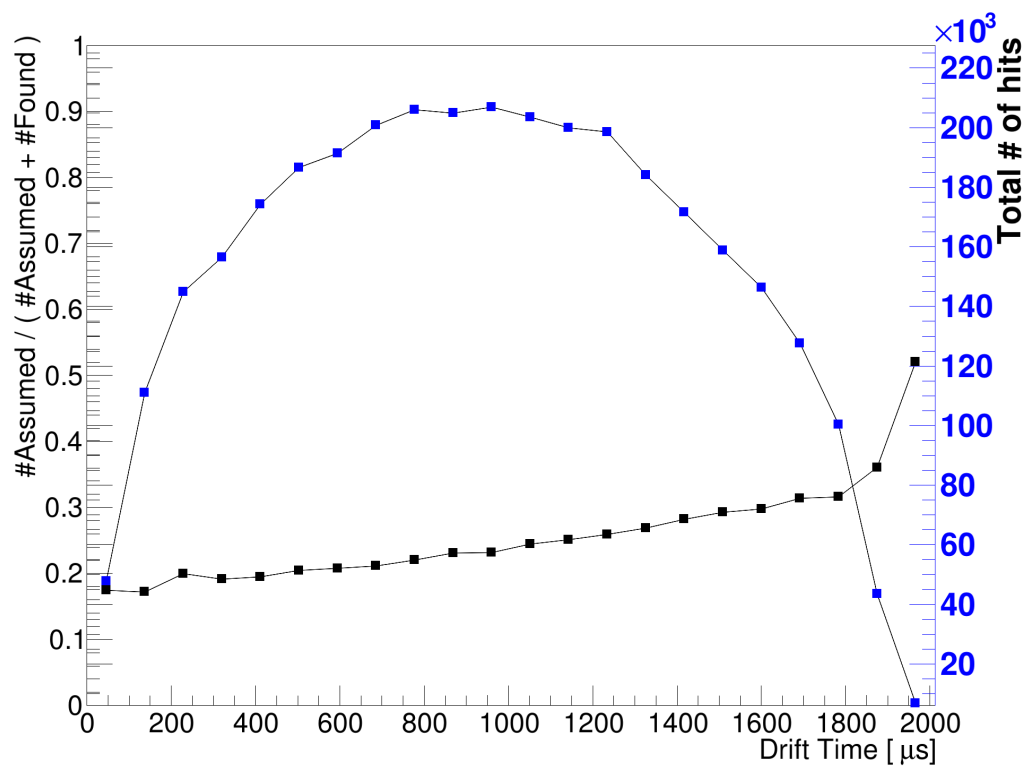

Fig. 6.3 Blue points: total number of hits included in each time bin over the full drift window. Black points: fraction of all hits which are "assumed". 


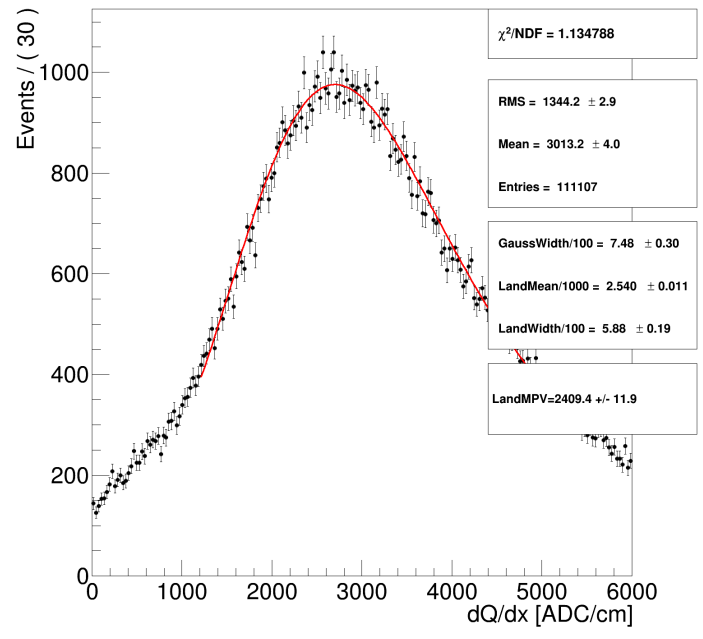

(a) Bin $1,91.5 \leq t<182.9 \mu \mathrm{s}$.

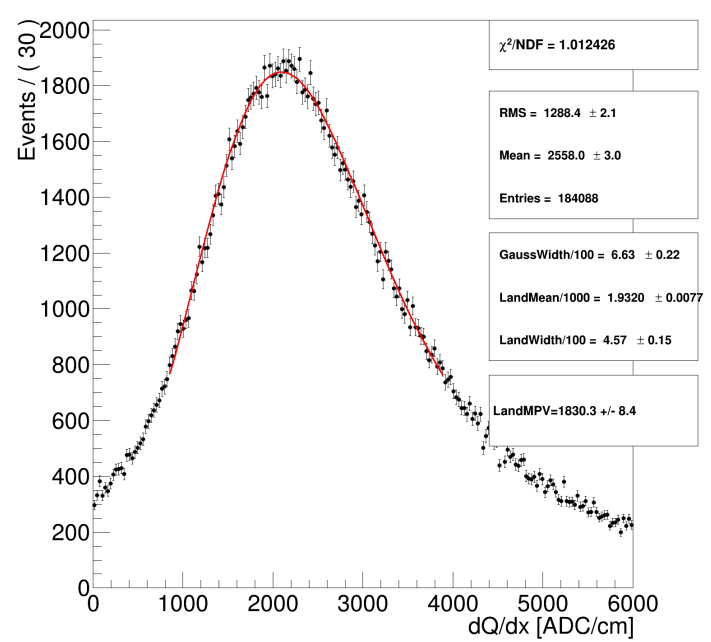

(c) Bin $14,1280 \leq t<1372 \mu s$.

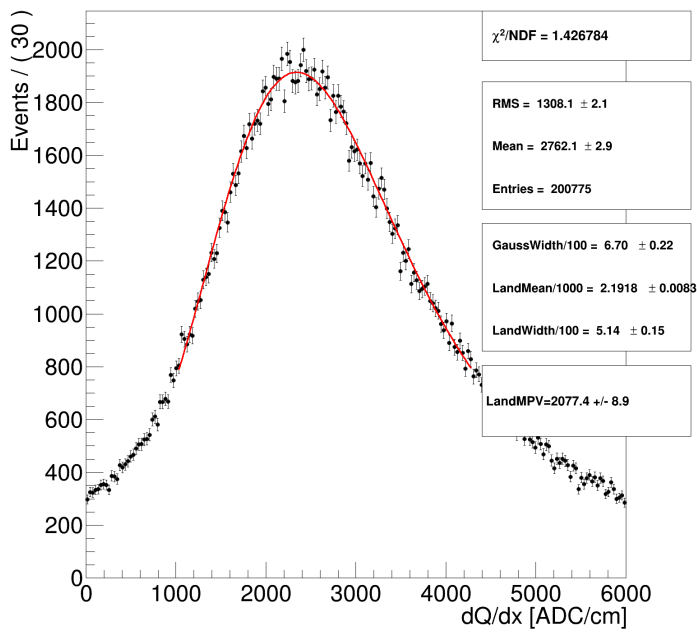

(b) $\operatorname{Bin} 7,640.2 \leq t<731.6 \mu s$.

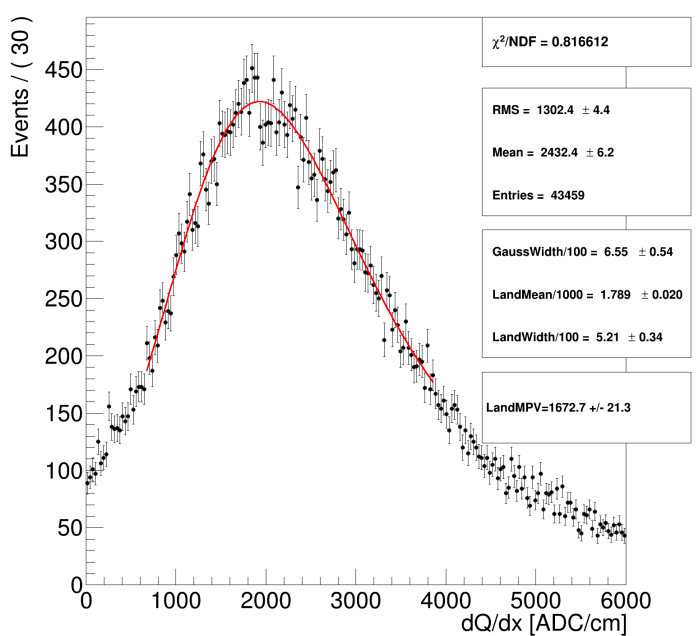

(d) Bin 20, $1829 \leq t<1922 \mu s$.

Fig. 6.4 Four (of 22 total) bins of $d Q / d x$ spanning different regions of the drift window. (a) and (d) correspond to hits originally near the anode and cathode, respectively. Landau $\otimes$ Gauss fits are shown as red curves, with best fit parameters included. 


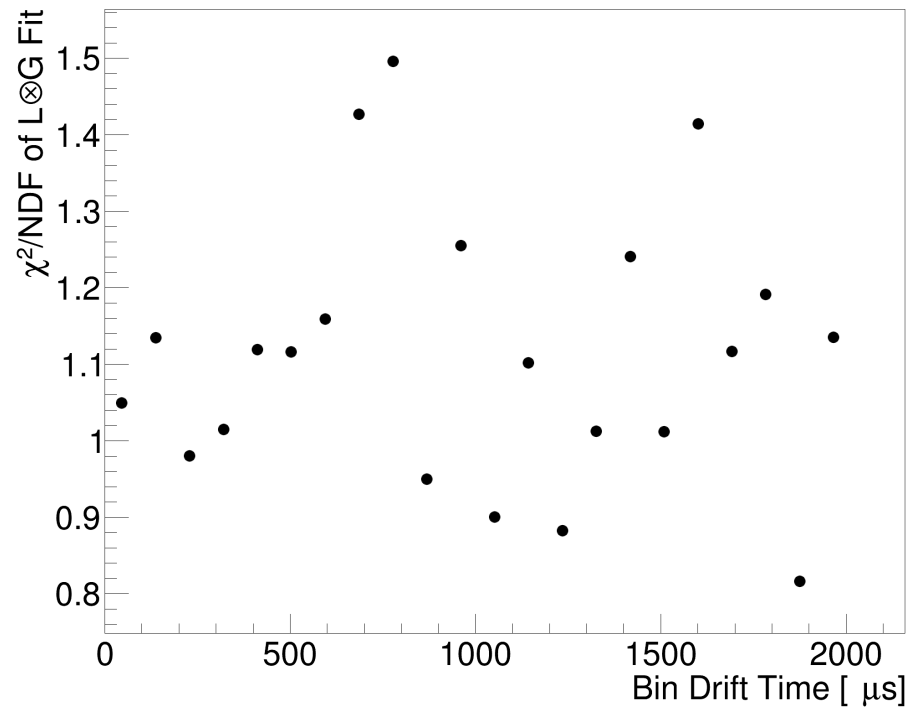

Fig. $6.5 \chi^{2} / \mathrm{NDF}$ of Landau $\otimes$ Gauss best fit curve to data.

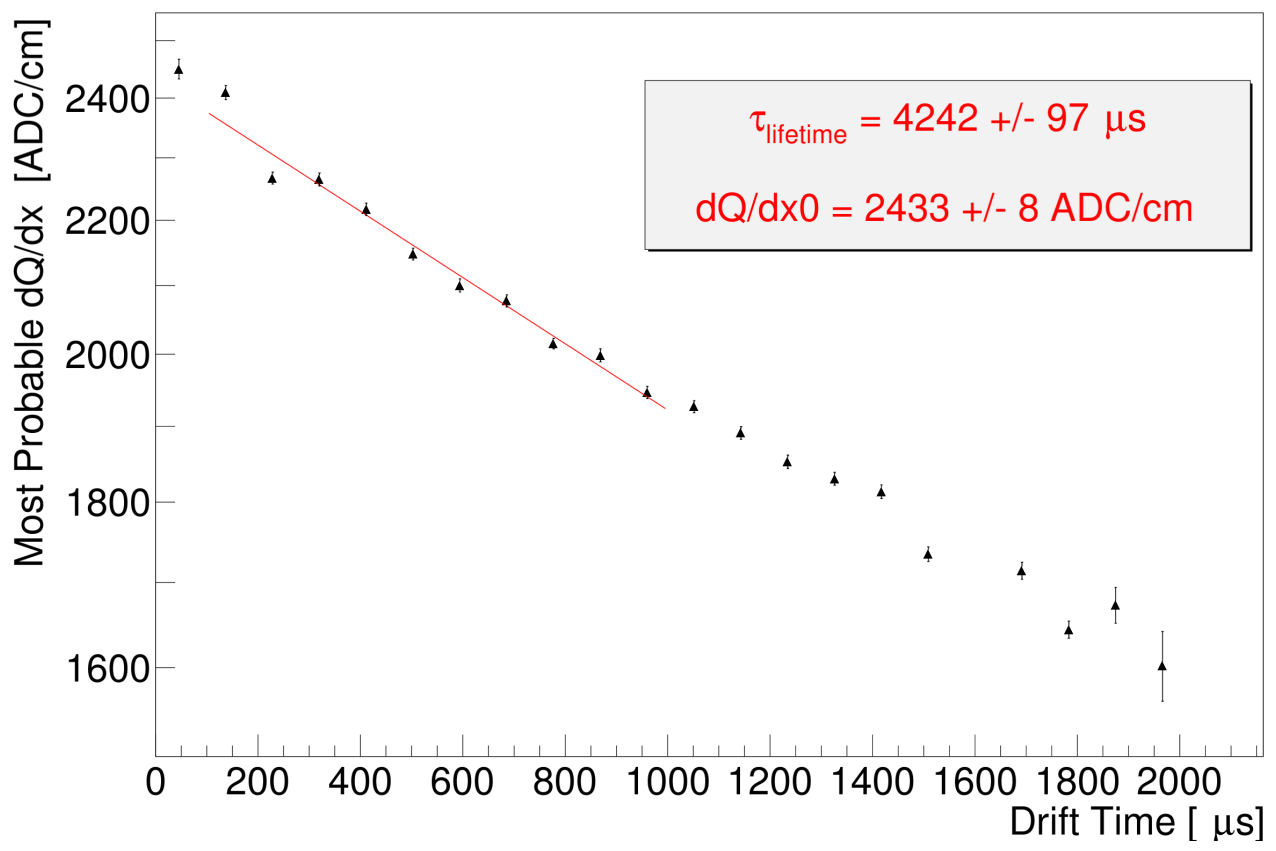

Fig. 6.6 Most probable hit $d Q / d x$ measured at the anode as a function of drift time, plotted on log scale. Exponential fit to fiducial range shown in red. 


\subsection{Simulation Reconstruction Performance}

The performance of the custom-developed reconstruction algorithms must be understood to understand the possible biases and systematic errors in the lifetime measurement presented above. There were many metrics used in evaluating the performance of the hit reconstruction for this electron lifetime analysis of the 35-ton detector. These metrics, when considered as a collective set of information, shed light on the biases alluded to at the beginning of this chapter. Within this context, the hit reconstruction efficiency and purity show how well the hit finder can determine whether an electronic pulse is a real ionisation charge hit or not. The hit charge efficiency and purity represent how well the hit reconstruction determines the correct hit charge. The detector resolution summarises the detector and reconstruction algorithms' combined response to noise and other factors.

To assess the performance of the reconstruction code at measuring true hits (as opposed to fake noise hits) and the accuracy to which hit charges are calculated, a module was written in LArSoft to compare the Monte Carlo Truth (MCT) information to the reconstructed hit ("Reco") information. The MCT information used was collected from the Geant 4 and detector simulation steps whereby the simulated ionisation charge in the detector along the particle track was associated with the detector channels which would be affected by such charges. So, each channel had a given true number of electrons at a particular time at which it interacted with the wire, given the drift velocity. This information was used to perform a reconstruction of MCT hits. To compare MCT hit charge (in units of [\# $\left.e^{-}\right]$) with the reconstructed hit integral (in units of [Integrated ADC]), a conversion was needed based on the particular characteristics of the 35-ton readout electronics:

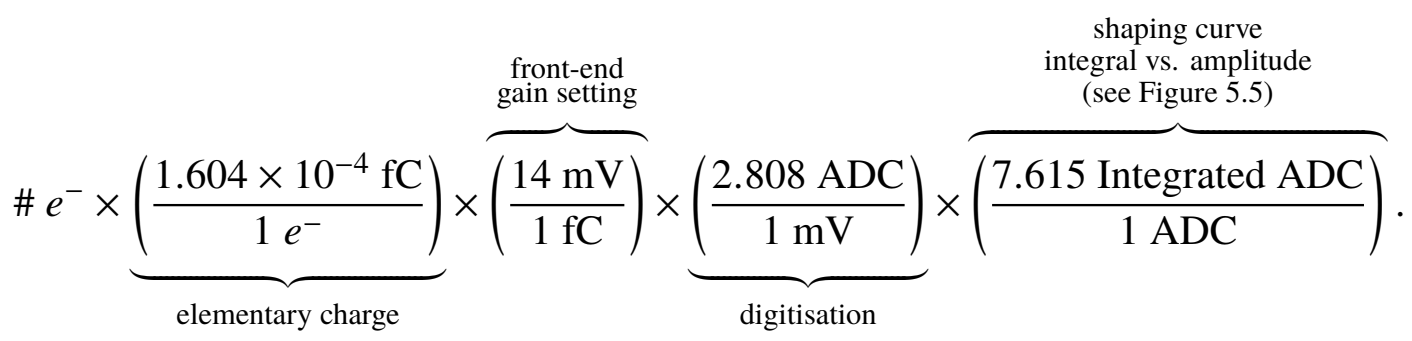

Reconstructed hits were compared with MCT hits by their relative waveform times and classified according to the truth table shown in Table 6.2. If at least one MCT hit was found between the start and end ticks of the reconstructed hit, the reconstructed hit was considered "true" and added to a collection of true positives (TP). Also, the charges of the associated MCT and reconstructed hits were collected and compared. If no MCT hit was found coincident with a reconstructed hit, that reconstructed hit was considered a false positive (FP), assumed to be either a noise pulse or background (non-primary muon) real charge hit. Finally, if there were 
MCT hits not associated with any reconstructed hits, these MCT hits were considered false negatives (FN), as the hit finder was not able to identify the pulse from the background noise. True negatives (TN) were not considered due to the infinite possibilities for successfully avoiding reconstructing hits which do not exist. The efficiency and purity statistics are then calculated:

$$
\text { Purity }=\frac{T P}{T P+F P}, \quad \text { Efficiency }=\frac{T P}{T P+F N} .
$$

\subsubsection{Simulated Samples}

It was expected that the primary factor which introduces bias in the measurement of electron lifetime was the level of noise in the detector. Therefore, simulated data sets were created for two different variables of detector parameters. Five different values of electron lifetime were simulated, ranging from $2.5 \mathrm{~ms}$ to $4.5 \mathrm{~ms}$ in $0.5 \mathrm{~ms}$ increments. To examine the bias as a result of noise, 16 different levels of the $\mu$ signal-to-noise ratio (S/N) scale factor (see Section 5.1.1.4 for the definition of $\mu$ ) were simulated, ranging from 0.5 to 2.0 in increments of 0.1 , for each value of the simulated lifetime. Therefore, 80 different simulated data sets were created for each parameter configuration, representing a wide range of possible scenarios for the real 35-ton detector. For each data set, around 35,000 events were simulated within the detector, giving sufficient statistics to measure the observed electron lifetime.

\subsubsection{Track Reconstruction Efficiency}

The track reconstruction efficiency is an essential metric in confirming the behaviour of the hit reconstruction in general. This efficiency is defined as the number of successfully reconstructed tracks (no cuts on track quality) divided by the number of muon coincidence triggers which are geometrically expected to produce a track in the detector. For most East-West muon counter coincidences, the particle tracks were guaranteed to have passed through the entire detector (apart from the very rare random coincidence trigger not due to a

Table 6.2 Truth table for comparing the existence of MCT and reconstructed hits.

\begin{tabular}{|c|c|c|c|c|}
\hline & & \multicolumn{2}{|c|}{ Reconstructed Hit } & \multirow{4}{*}{ Efficiency $=\frac{T P}{T P+F N}$} \\
\hline & & Yes & No & \\
\hline \multirow{2}{*}{ MCT Hit } & Yes & True Positive (TP) & $\begin{array}{c}\text { False Negative }(\mathrm{FN}), \\
\text { Type II Error }\end{array}$ & \\
\hline & No & $\begin{array}{c}\text { False Positive (FP), } \\
\text { Type I Error }\end{array}$ & True Negative (TN) & \\
\hline
\end{tabular}


single particle interaction), and were expected to be reconstructable. The track efficiency was affected by tracks which did not pass through the detector, as was the case with a few coincidences where the counters straddled the edges of the TPC, and by events which had high noise preventing the track from being successfully reconstructed.

Because the track efficiency depends on the muon counter coincidences, it is shown separately for every possible trigger. Figure 6.7 shows the track finding efficiency for the "nominal" simulated data set with electron lifetime of $3.5 \mathrm{~ms}$ and a $\mu$ parameter of 1.0. For the counter triggers which did not straddle the TPC edges, there was consistently greater than $99 \%$ efficiency. Thus, there was no reason to believe that any significant biases were introduced to the electron lifetime analysis due to the complete inability of a track to be reconstructed.

\subsubsection{Hit Reconstruction Efficiency and Purity}

The efficiency and purity (Equation 6.7) of hit finding are two primary metrics which were used to determine how well the reconstruction performed given the particular characteristics of signal and noise. They must each lie in the range 0 to 1 . In words, the hit finding efficiency represents the fraction of all MCT hits which are reconstructed. For example, a high efficiency would mean that the reconstruction found most of the real hits that exist. Similarly, the hit finding purity represents the fraction of all reconstructed hits which are associated in time with MCT hits. For example, a high purity would mean that, of the hits which are reconstructed, most of them were real hits. These measurements depend on many different aspects of the data, including $\mathrm{S} / \mathrm{N}$, drift distance of the charge packets, electron lifetime of the LAr, and track angle with respect to the APA plane, among others.

While the definitions of efficiency and purity are similar, they often have conflicting behaviours when improving and tuning the reconstruction. For example, by lowering the hit finding threshold, the hit finder is more likely to detect more of the true charge deposits which increases the efficiency, but at the same time, more fake noise pulses are reconstructed as hits, thereby reducing the purity. An ideal reconstruction maximises both, which usually requires delicate balancing.

In practice, the efficiency, $\epsilon_{S}$, and purity, $\phi$, of hit finding are calculated by the following formulae:

$$
\begin{aligned}
\epsilon_{S} & =\frac{\sum_{e} \mathrm{TP}_{e}}{\sum_{e} \mathrm{TP}_{e}+\sum_{e} \mathrm{FN}_{e}}, \\
\phi & =\frac{\sum_{e} \mathrm{TP}_{e}}{\sum_{e} \mathrm{TP}_{e}+\sum_{e} \mathrm{FP}_{e}}
\end{aligned}
$$




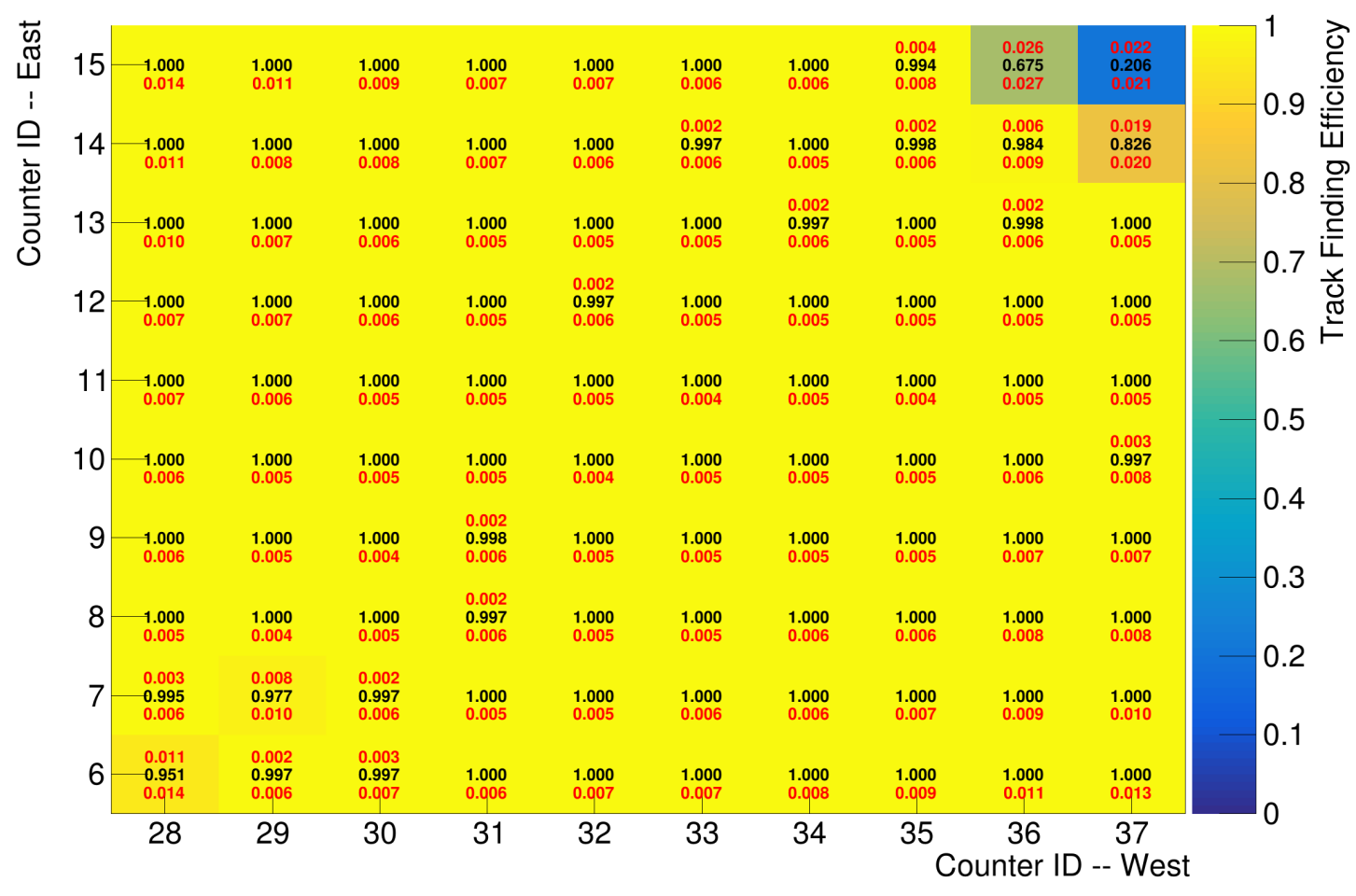

Fig. 6.7 Track finding efficiency (black text) and associated upper and lower binomial errors (red text) for all possible East-West muon counter coincidences in the simulated data set (3.5 ms electron lifetime, $1.0 \mu$ parameter). 
where the sums are over all $e$ events considered. The errors on these uncertainties are calculated by considering the efficiency and purity as a probability of a positive outcome of a Bernoulli trial [177]. Therefore, the likelihood function is given by the binomial distribution:

$$
P(k \mid \rho, N)=\frac{N !}{k !(N-k) !} \rho^{k}(1-\rho)^{N-k}
$$

where $P(k \mid \rho, N)$ represents the probability that $k$ events pass the cut, given that the true efficiency or purity statistic is $\rho=\epsilon_{S}, \phi$ and that there are $N$ events in the sample. Therefore, the probability of the true $\rho$ statistic, given the observed $k$ and $N$, is determined using Bayes' theorem:

$$
P(\rho \mid k, N)=\frac{1}{Z} P(k \mid \rho, N) \times P(\rho \mid N)
$$

where $Z$ is a normalisation constant and $P(\rho \mid N)$ is a prior probability assigned for the true $\rho$ to be found. In this simulation, the prior probability is uniform on the range 0 to 1 , and zero otherwise. Under these assumptions, the probability of the true $\rho$ given $k$ and $N$ is then

$$
P(\rho \mid k, N)=\frac{\Gamma(N+2)}{\Gamma(k+1) \Gamma(N-k+1)} \rho^{k}(1-\rho)^{N-k}
$$

which is a beta function. Under the uniform prior, this probability simplifies to $k / N$, which is represented in Equations 6.8 and 6.9. However, the errors on these values are calculated as the upper and lower 68\% intervals of the beta distribution in Equation 6.12. For this analysis, these intervals are calculated automatically within the TEfficiency: : Bayesian method in ROOT.

The efficiencies for all datasets are summarised in Figure 6.8 which shows the strong dependence of hit finding efficiency on the detector noise in the range from 0.5 to 2.0 times the 35-ton $\mathrm{S} / \mathrm{N}$. As expected, as the detector noise decreases ( $\mathrm{S} / \mathrm{N}$ and $\mu$ both increase), the hit finding efficiency increases significantly. Looking forward, given the steps already taken to model the 35-ton detector noise, it is expected that even small errors in modelling the 35-ton signals will significantly impact the reconstruction efficiency, purity, and overall analysis biases. The efficiency is also improved by longer electron lifetimes because there is less charge attenuation over the drift, leaving more charge for detection and reconstruction.

As was described in Section 5.3.3, the RHF increased the hit finding purity, $\phi$, by making assumptions on the event topology and source ionising particles through the implementation of the straight track finding algorithm. The overall effectiveness of these steps is shown clearly in Figure 6.9 where, for all simulated datasets, the hit finding purity is consistently greater than $94 \%$. The purity does not reach $100 \%$ for any of the simulated datasets, which 


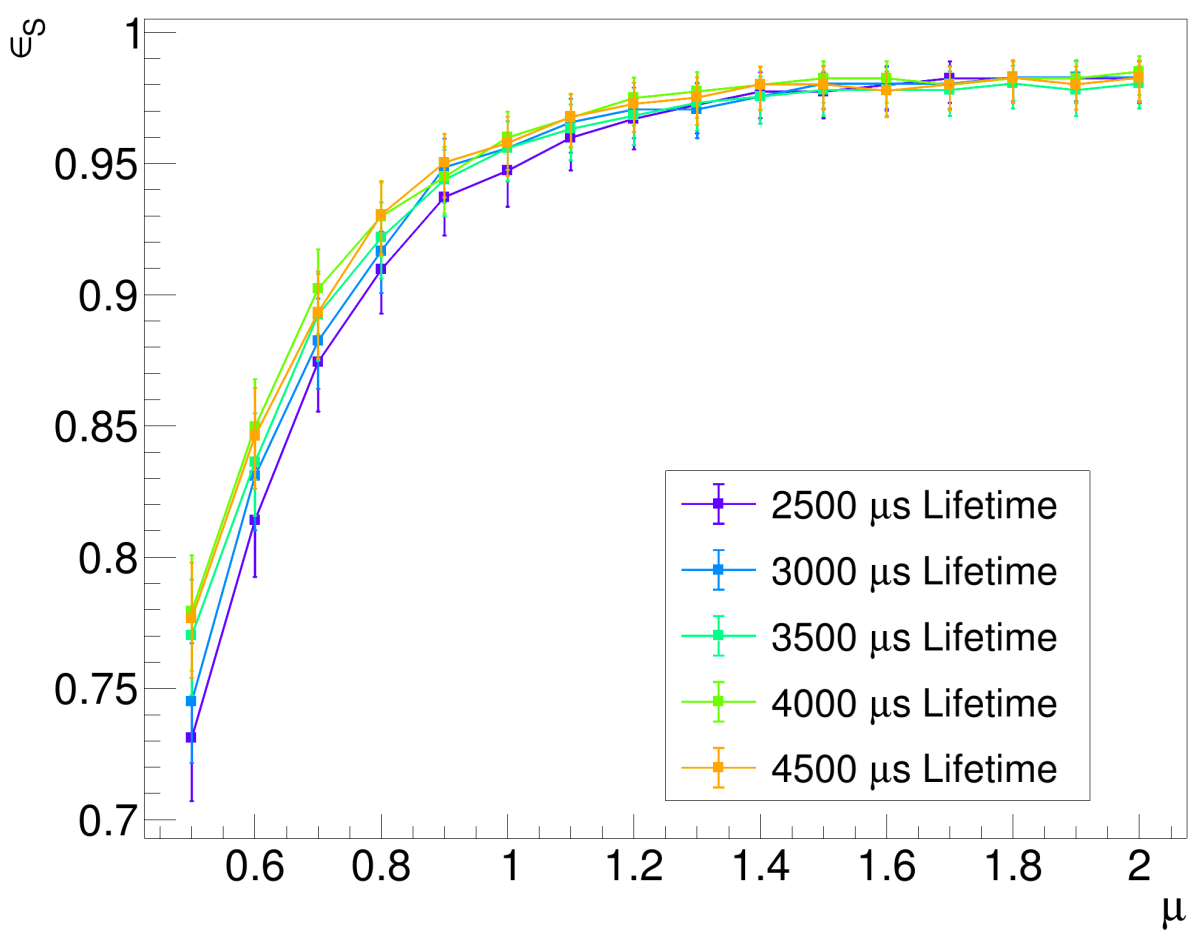

Fig. 6.8 Efficiency of hit finding as a function of $\mu$ for muon counter triggers 9 and 31 (44 $\pm 15 \mathrm{~cm}$ drift distance) and several values of $\tau_{\mathrm{MC}}$.

is assumed to be accounted for by the misassociation of delta electron tracks as part of the straight muon tracks.

The hit finding efficiency and purity also depend on drift distance, or equivalently, drift time. As shown in Figure 6.10, the efficiency decreases with drift distance as charge loss to impurities causes smaller amplitude signals from ionisation at long drift times. The relationship between efficiency and drift distance implies there is a benefit to applying a fiducial cut to the hits collected. That is, by setting a requirement that the hit finding efficiency must be higher than, say, 90\%, hits from a majority of the detector will be ignored, as there is not enough confidence that a representative sample of the $d Q / d x$ distribution is used. Deciding what this efficiency cut should be is arbitrary, and can lead to different conclusions altogether. The fiducial cuts applied in the analysis are discussed further in Section 6.4.5.

The hit finding purity also depends on drift distance, although to a much lesser extent than the efficiency. As stated earlier, in nearly all simulated datasets, the hit finding purity is close to ideal. Figure 6.11 shows the purity is consistently well above $90 \%$, regardless of drift distance, simulated electron lifetime, or $\mu$. No fiducial cuts based on the hit finding purity are seen as necessary or beneficial in reducing bias in the analysis. 


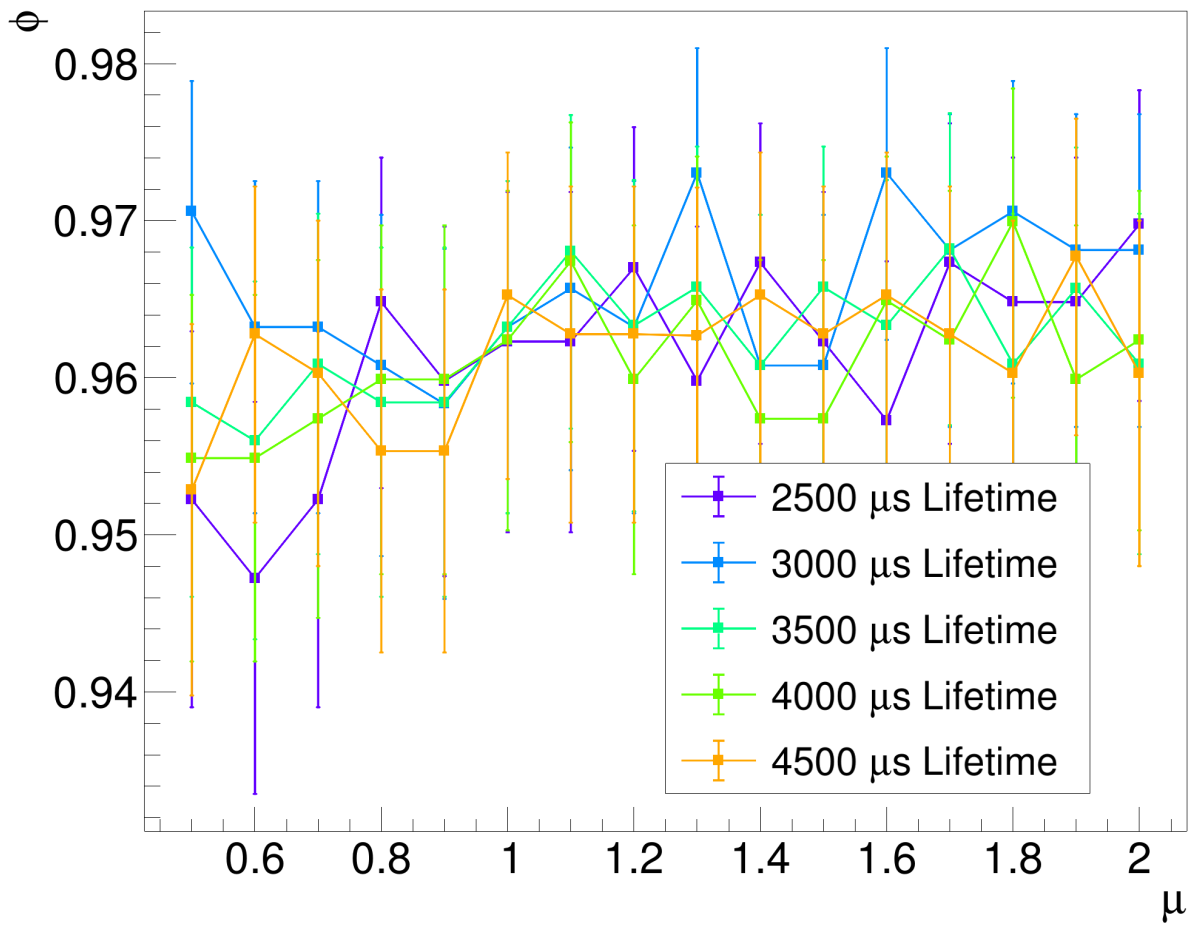

Fig. 6.9 Purity of hit finding as a function of $\mu$ for muon counter triggers 9 and $31(44 \pm 15 \mathrm{~cm}$ drift distance) and several values of $\tau_{\mathrm{MC}}$.

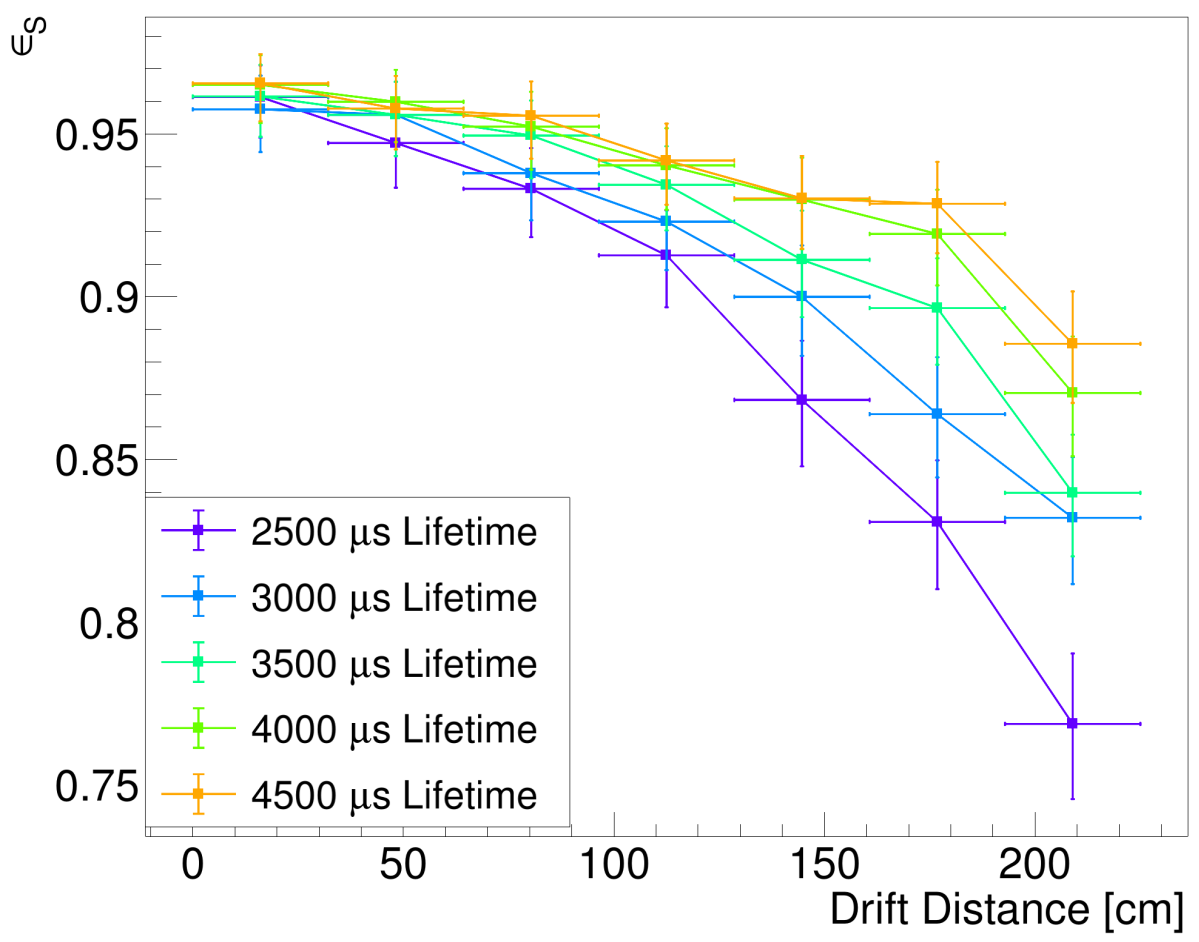

Fig. 6.10 Efficiency of hit finding in the simulation as a function of drift distance for $\mu=1.2$ and several values of $\tau_{\mathrm{MC}}$. 


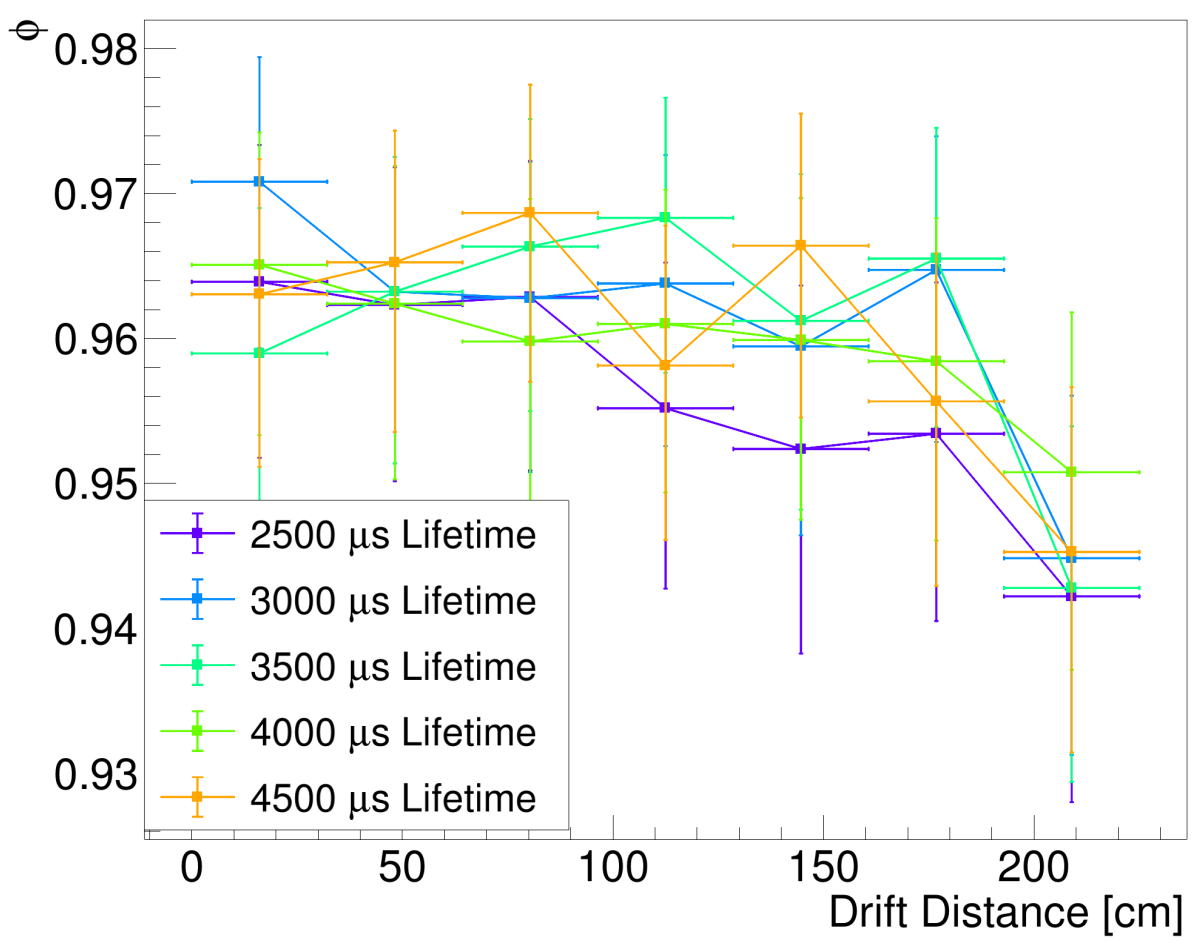

Fig. 6.11 Efficiency of hit finding in the simulation as a function of drift distance for $\mu=1.2$ and several values of $\tau_{\mathrm{MC}}$.

By separating out events based on the coincident muon counters, the dependence of hit finding efficiency and purity on track angle and drift distance is seen. Figure 6.12 shows the efficiency and error of the events for every possible muon counter coincidence in a representative data set, $3.5 \mathrm{~ms}$ lifetime and a $\mu$ parameter of 1.0 (counter ID numbers are referenced in Figure 6.13). Some interesting features are seen, apparent in the bottom left of the plot, where the efficiency is decreased. These counter coincidences correspond to events which cross TPC boundaries and are not entirely contained within the TPC over the entire range of $z$. Therefore, the hit finding efficiency is reduced. Also, the top right bin corresponds to events which are mostly outside of the TPC, leading to a nearly zero hit finding efficiency as very few of the hits exist within the TPC. The diagonals in this plot are also interesting. For example, the bottom left to top right diagonals correspond to events with equal track angles with respect to the APA. The top left to bottom right diagonals represent events with equal mean drift distances but different track angles, which are interesting because they show very little dependence on track angle with respect to the APA. The independence of hit finding efficiency and the track angle is not surprising as the analysis is performed on the hit level, not the track level. 


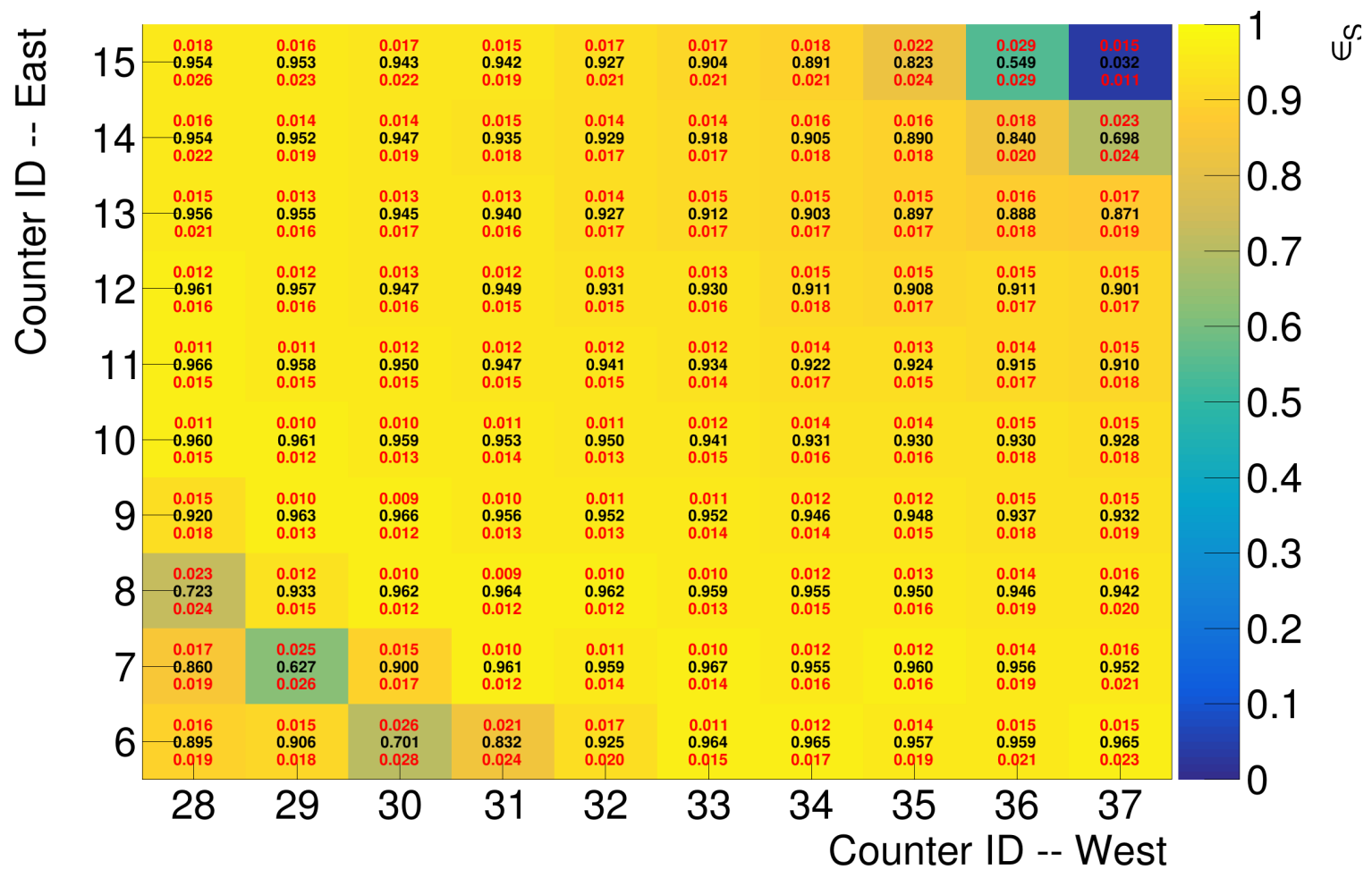

Fig. 6.12 Hit finding efficiency (black text) and associated uncertainty (red text) for events of all possible combinations of East-West counter coincidences ( $3.5 \mathrm{~ms}$ simulated lifetime and $1.0 \mu$ parameter).
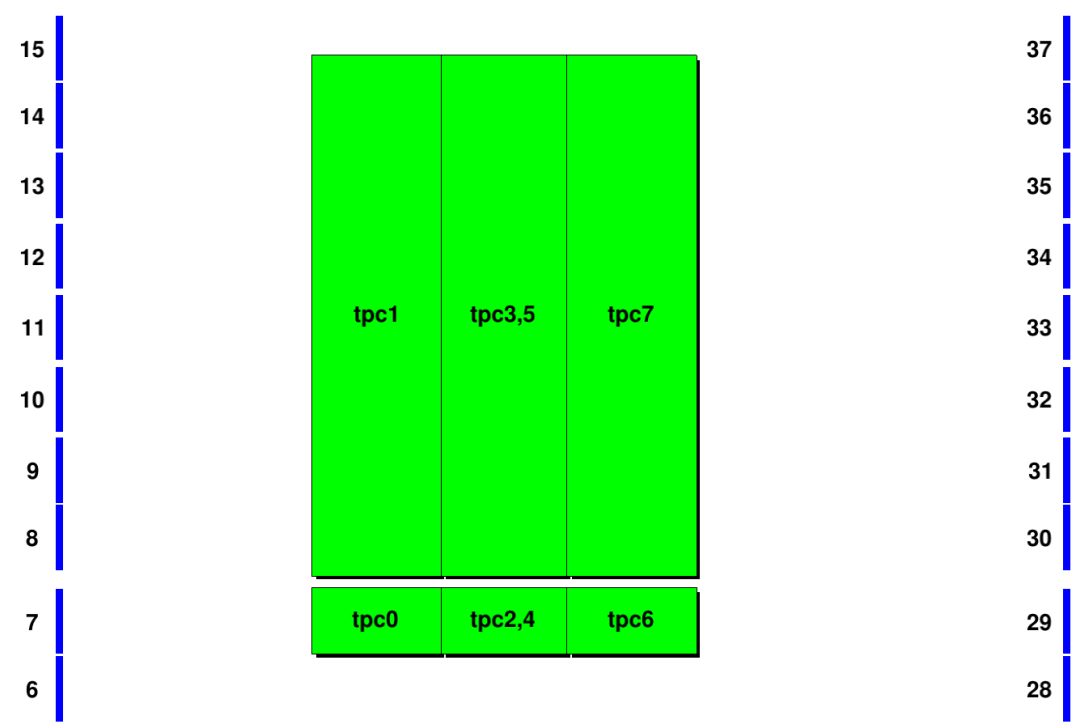

Fig. 6.13 Relative locations of East-West muon counters and the detector. 
The hit finding purity for all muon counter coincidences, shown in Figure 6.14, reveals what is expected from the previous plots of purity. Nearly perfect hit finding purity is shown for nearly all events, regardless of track angle, drift distance, or counter coincidence, except when the event is not contained within the detector.

To better apply the measurement of hit finding efficiency to the determination of the analysis bias, the efficiency must be known with respect to the hit charge. By combining the information gained in the previous plots in this section, it is expected that smaller charge hits will be more difficult to reconstruct, and therefore will be less likely to be found within the noise at all. Indeed, Figure 6.15 shows that the hit finding efficiency decreases with hit charge. The efficiency for small charge hits $\left(Q_{\mathrm{MC}}<1000\right)$ goes toward zero, as the hits fall below the hit finding threshold. For larger charge hits, there are much fewer statistics as the Landau tails off, but the efficiency tends toward 100\%, as expected. The plot also shows a histogram of $Q_{\mathrm{MC}}$ indicating that the Landau most probable hit charge lies very close to the efficiency cutoff. The cause of the short plateau in the $\mu=0.5$ graph at 1250 ADC in Figure 6.15 is unknown, but it is suspected to be an artefact of delta electron contamination in the high noise sample.

\subsubsection{Charge Resolution and Accuracy}

Accurate hit charge measurement is vital to any calorimetric measurement as the calculation of the number of ionisation electrons must be accurately reconstructed. Several metrics are used to compare the reconstruction of hit charge to the "true" hit charge. First, the correlation between the MCT hit charge and the RHF reconstructed charge is shown in Figure 6.16. The correlation coefficient of these two charge measurements is $r=0.999$, showing close agreement. The wide band on the profile histogram refers to the standard deviation of the hit charges in each bin, indicating the spread of values. The loss in hit finding purity for very small $Q_{\mathrm{MC}}$ hits is seen in this plot as the hook-like behaviour at low MCT charge where the reconstructed charge is consistently higher than the true charge. This is a result of several issues in reconstruction, including the THB step in the reconstruction where hit locations are assumed based on hits on adjacent wires. In these cases, the hit charge measured could be positive fluctuations in the wire noise, appearing as a reconstructed hit where there is no actual charge deposition. In any case, the reconstructed hit charge for hits with integral less than $200 \mathrm{ADC} / \mathrm{cm}$, or so, cannot be assumed to be accurate. 


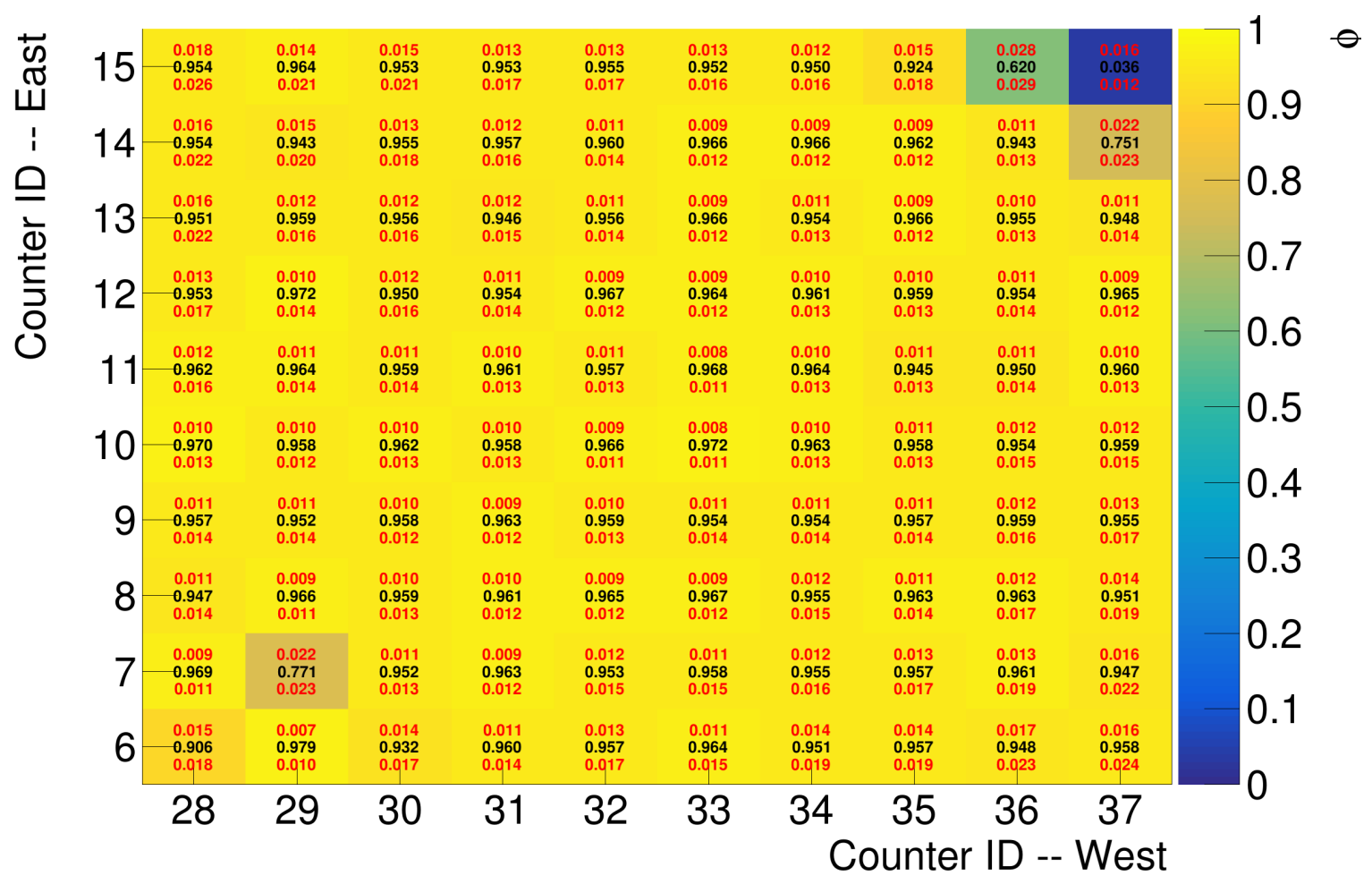

Fig. 6.14 Hit finding purity (black text) and associated uncertainty (red text) for events with all possible combinations of East-West counter coincidences ( $3.5 \mathrm{~ms}$ simulated lifetime and $1.0 \mu$ parameter). 


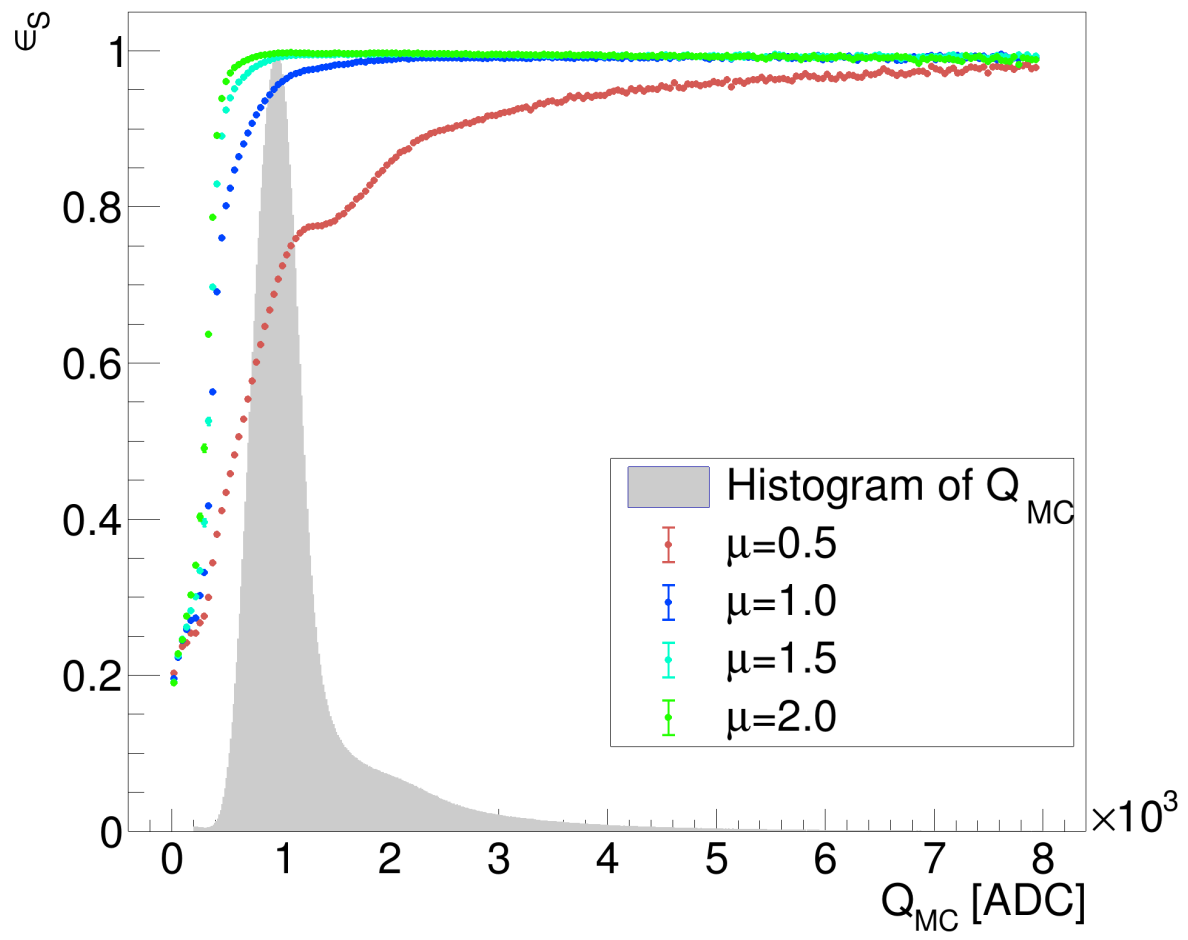

Fig. 6.15 Hit finding efficiency versus MCT hit charge for several values of $\mu$. The histogram (arbitrary normalisation) of $Q_{\mathrm{MC}}$ is shown for comparison on the same horizontal scale.

The charge residual, $R_{q}$, is another valuable metric for determining the performance of hit reconstruction. For this analysis, the charge residual is defined by

$$
R_{q}=\frac{Q_{\mathrm{Reco}}-Q_{\mathrm{MC}}}{Q_{\mathrm{MC}}}
$$

and shown in Figure 6.17. $R_{q}$ is interpreted as the accuracy of the hit reconstruction. For example, $R_{q}=0$ implies that the reconstructed hit has the same amount of charge as the true MCT hit-a perfect reconstruction. Deviations from this imply that charge was reconstructed differently than the charge that was simulated, an error in reconstruction. Figure 6.18 shows the mean standard residual for each bin of $Q_{\mathrm{MC}}$. Here, in the relevant range of $Q_{\mathrm{MC}}$ for the Landau MPV (around 1000 ADC), the hit charge reconstruction differs from the true charge by about $-2 \%$ for $\mu=1.0$.

The resolution, $\sigma_{q}$, is defined as the full width at half maximum of the residual histogram in Figure 6.17, and relates to the spread of residuals. The charge resolution with respect to the MCT hit charge in Figure 6.19 shows that for low charges, the resolution dramatically 


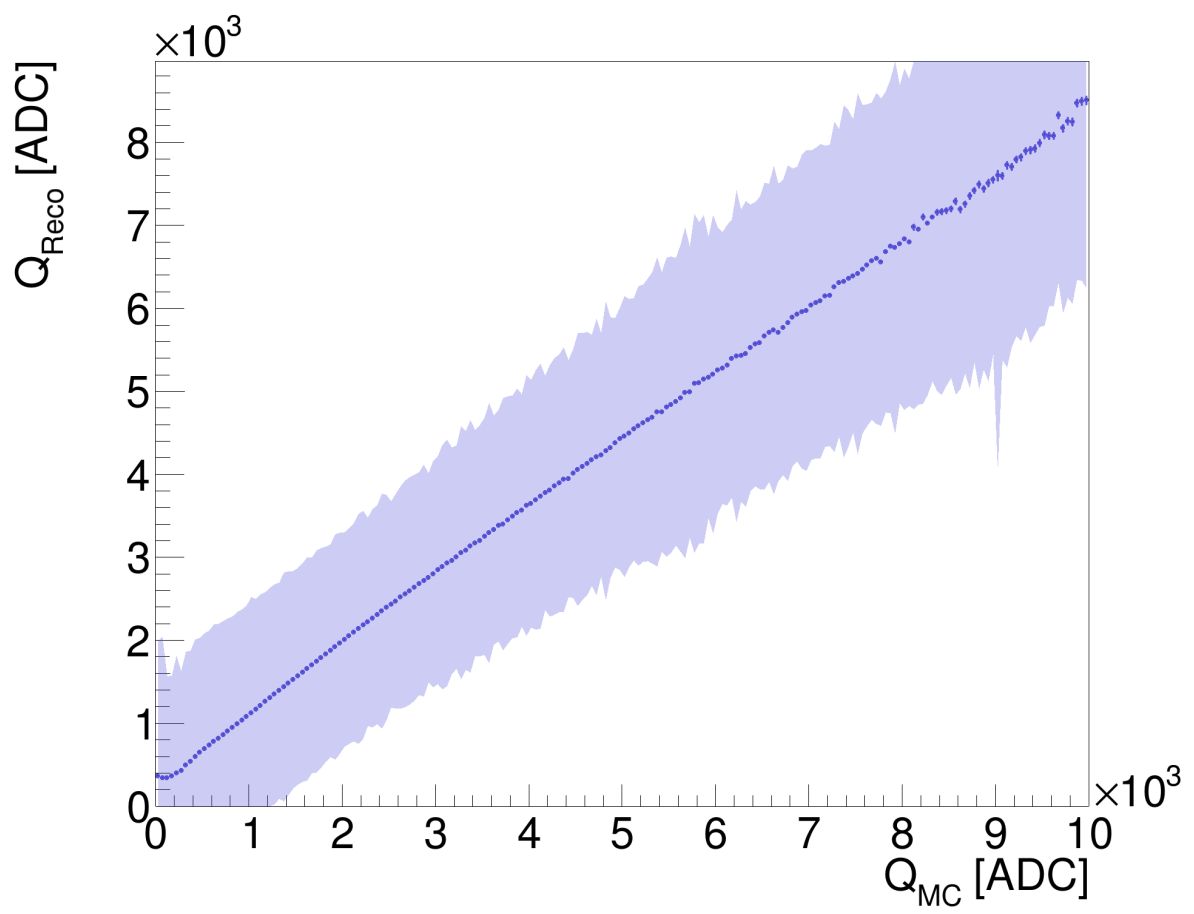

Fig. 6.16 Profile histogram of reconstructed hit charge given associated MCT hit charge. Error bars represent the standard error on the mean, the shaded region represents the standard deviation.

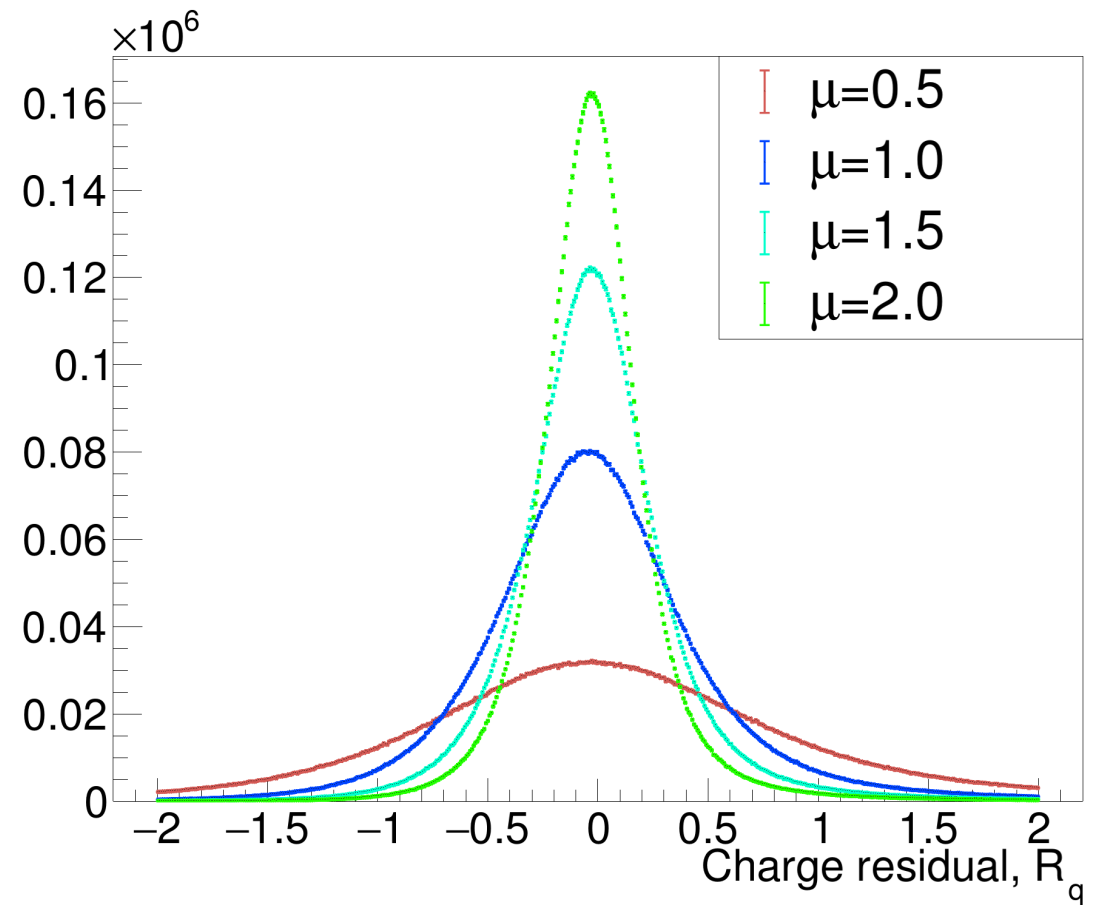

Fig. 6.17 Histogram of charge residuals, $R_{q}$, for several values of $\mu$. 


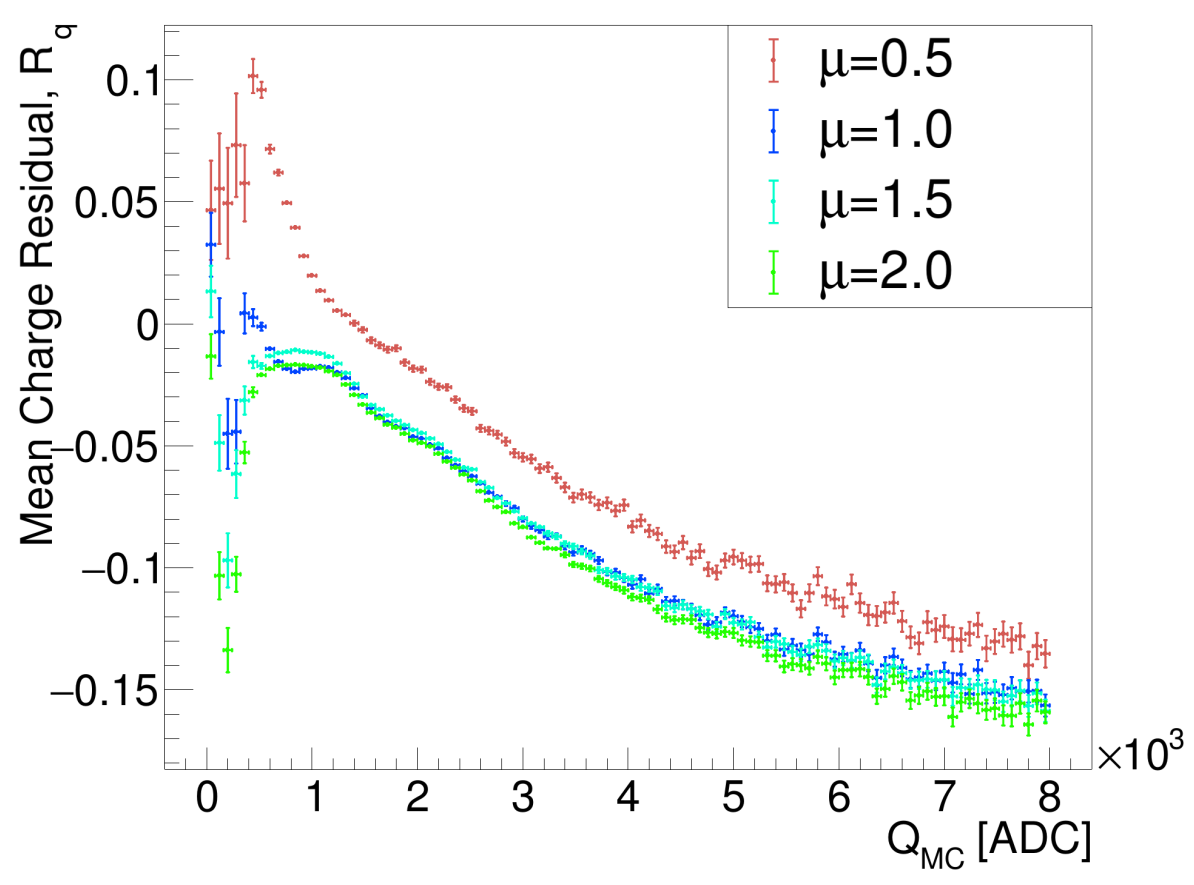

Fig. 6.18 Mean charge residual $R_{q}$ versus $Q_{\mathrm{MC}}$ for several values of $\mu$.

worsens, regardless of $\mu^{1}$. For $Q_{\mathrm{MC}}$ in the relevant region for the Landau MPV (about $1000 \mathrm{ADC}$ ), the charge reconstruction resolution is about $40 \%$ for $\mu=1.0$.

\subsubsection{Discussion of charge resolution}

The most striking thing about the charge resolution plot in Figure 6.19 is not the absolute values of the resolutions. It is that the charge resolution varies from roughly $70 \%$ to $30 \%$ in the range $400<Q_{\mathrm{MC}}<1500 \mathrm{ADC}$ for $\mu=1.0$, the relevant range of charges for the argon electron lifetime analysis. The varying charge resolution has significant implications on the method of analysis attempted in Section 6.1.1. In particular, the analysis assumes a model of hit charge to be Landau convoluted with a single Gaussian. The Gaussian is expected to account for all possible sources of uncertainty in calculating the hit charge including thermal diffusion fluctuations, digitisation response of the readout system, hit finding efficiency, electronics noise influence on hit reconstruction, or even microfluctuations in the electric field. The single Gaussian response is convoluted with the Landau across the entire range of hit charges, which is an inaccurate model as the charge resolution is dependent on the hit charge.

\footnotetext{
${ }^{1}$ Note that because $Q_{\text {Reco }}$ is allowed to be negative, resolutions greater than $100 \%$ are possible.
} 


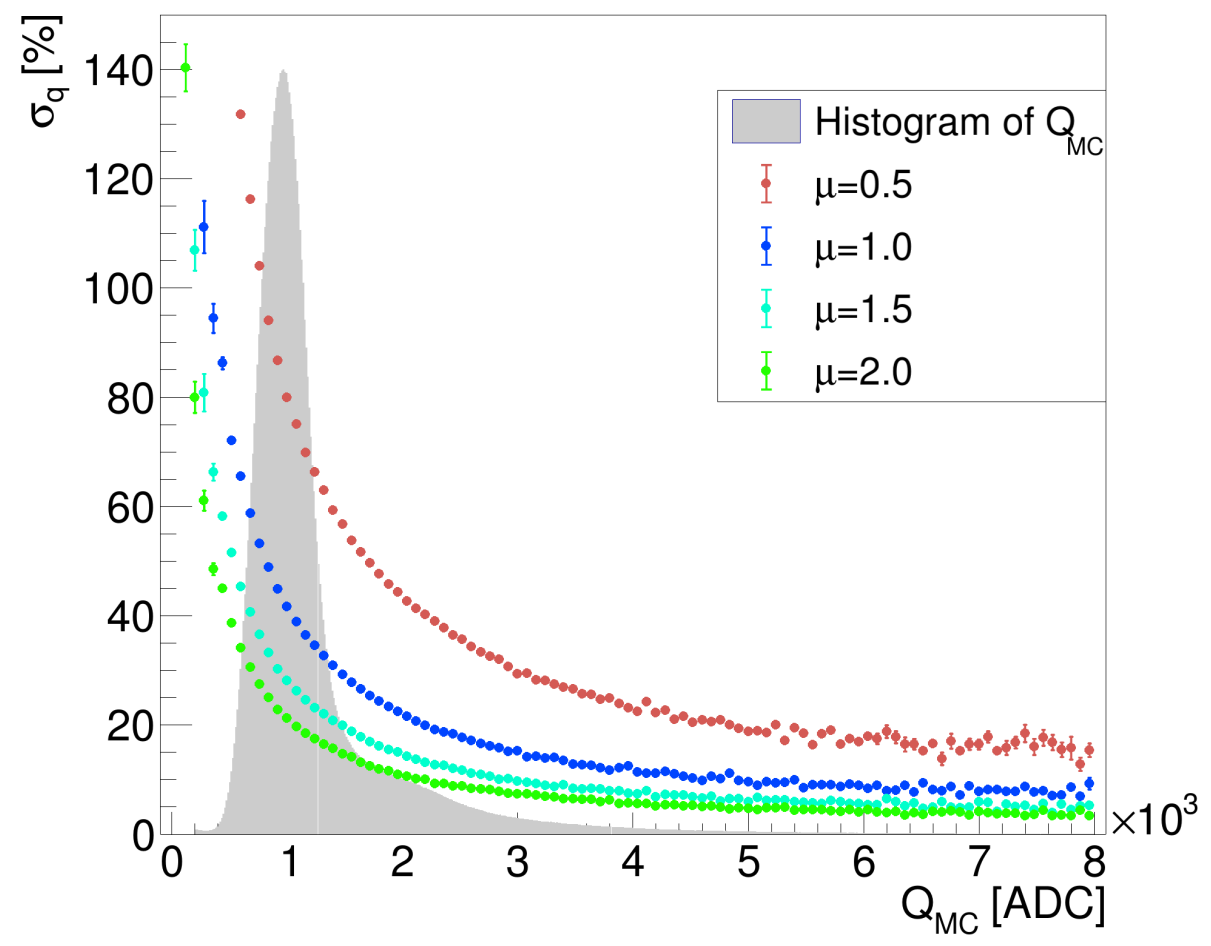

Fig. 6.19 Charge resolution vs. hit charge for several values of $\mu$. The histogram (arbitrary normalisation) of MC hit charge is shown in shaded grey for comparison.

An ideal calorimetric analysis will take all of these nonlinear fluctuations to the overall detector response into account when deconvolving the Landau form of particle energy loss in the detector from the detector response. While an ideal raw signal deconvolution would remove some of these effects, it is seen to introduce other biases to the hit charges at the same time. Especially with the 35-ton noise characteristics, the deconvolution and noise removal has not been fully developed and does not use a 35-ton-specific model of signal shape for the particular electronics. Also, the single Gaussian response function would have to be replaced by a charge-dependent model, introducing more variables into the $\mathrm{L} \otimes \mathrm{G}$ convolution.

The consequences of this model-dependent bias were not studied in detail. However, for this analysis, the $\mathrm{L} \otimes \mathrm{G}$ model described in Section 6.1.2 was retained, despite the possible uncertainties associated with the modelling of the binned charge distributions. It is assumed that any resulting bias present in the data was also reproduced in the simulation. Therefore, it is essential for the simulation to represent the collected 35-ton data accurately. 


\subsection{5-ton Data Reconstruction Performance}

As with the analysis of simulation reconstruction performance, the hit and track finding efficiencies of 35-ton data were calculated to shed light on the uncertainties in the electron lifetime measurement. However, since the true hit charge, hit time, and particle identification are not known a priori in data, some assumptions must be made on the event topology and geometry of the detector.

\subsubsection{Track Reconstruction Efficiency}

As in Section 6.2.2, for the full 35-ton data set, the track reconstruction efficiency by counter coincidence is shown in Figure 6.20. For all coincidences which guarantee tracks in the detector due to geometry, there is greater than $98 \%$ track finding efficiency.

\subsubsection{Hit Reconstruction Efficiency}

The measurement of hit reconstruction efficiency for 35-ton data could not be completed in the same way as in the simulation, as there was no "truth" information to compare. Nevertheless, by making several minor assumptions about the expectation of hits to exist in the detector, measurement of the efficiency was still made. The premise for the efficiency measurement for data, which is based on the same assumption which motivates the use of the THB algorithm in the reconstruction, is that the ionisation events in the detector leave continuous mostly straight deposits of charge throughout the entire detector from East to West. Thus, it is expected that every single vertical collection plane wire signal in the given TPC volume will be subject to current induced by drifting charge, with one specific exception explained below. By eliminating those wires which were expected to give no signal (because they were included in the bad channel list, or physically impossible due to the detector geometry), an efficiency, $\epsilon_{D}$ was calculated,

$$
\epsilon_{D}=\frac{\text { Number of wires with at least one hit reconstructed }}{\text { Number of non-bad wires }} .
$$

To illustrate the calculation of real hit efficiencies, a cartoon diagram of a typical East-West triggered muon event is shown in Figure 6.21 where the detector $z$ coordinate and wire number increase from right to left, and the $y$ coordinate increases from bottom to top. The triggered muon track is represented by the red line and passes through two muon counters, which are always located at the same $y, z$ coordinates. The white hatched boxes represent the TPC drift volumes, and the vertical hatches themselves represent the direction of collection 


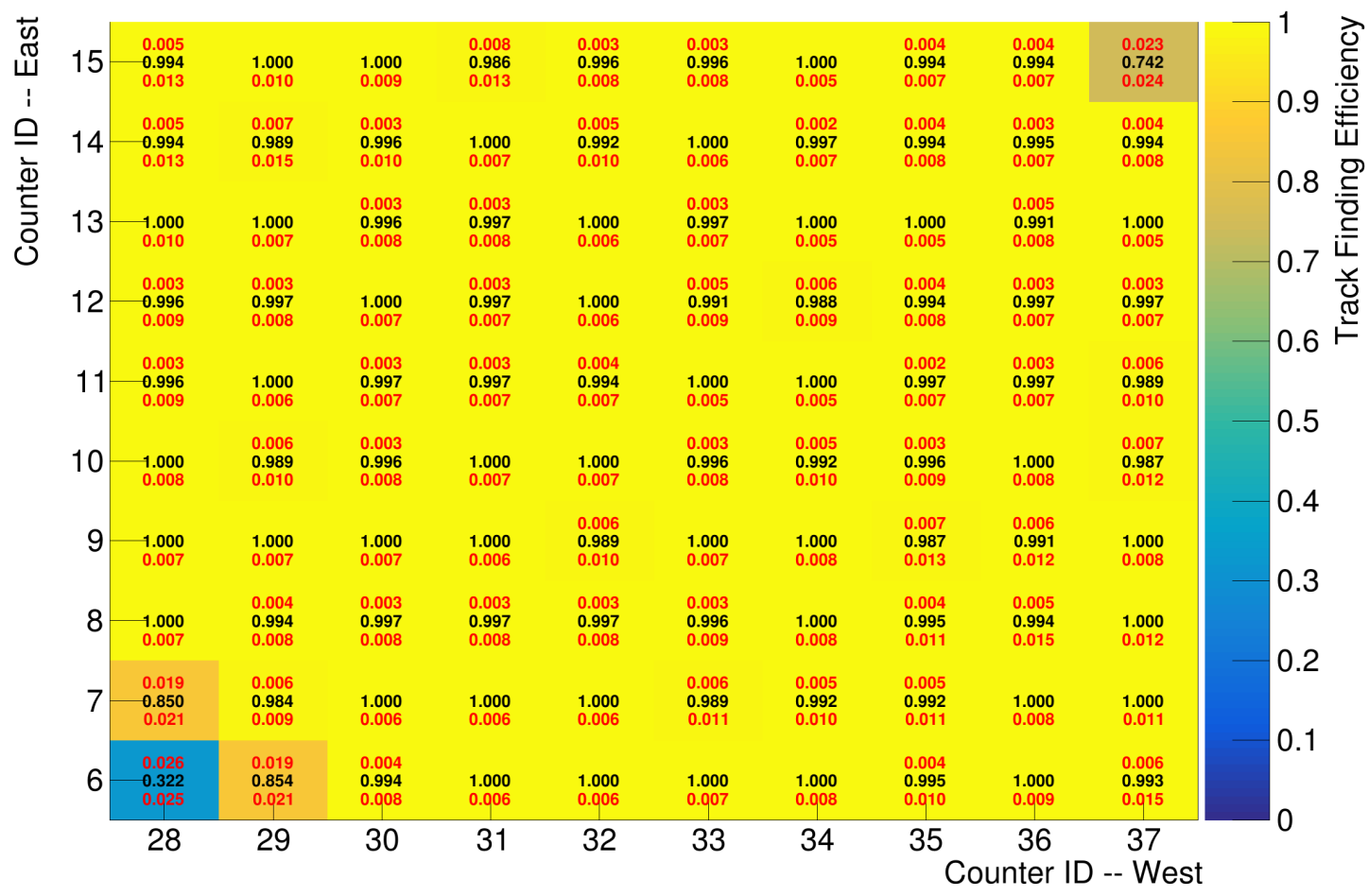

Fig. 6.20 Track finding efficiency (black text) and associated upper and lower binomial errors (red text) for all possible East-West muon counter coincidences in the full 35-ton data set. 
plane wires on each APA. For each of the tall APAs (in TPCs 1 and 7 in the diagram, but also TPCs 0 and 6 for the short drift), every straight track crosses every collection plane wire. So, apart from the bad wires, every wire in these APAs should record a charge pulse. Thus, the hit finding efficiency in these TPCs is straightforward to calculate, as given in Equation 6.14.

For TPCs 2, 3, 4, and 5, which are stacked vertically making a horizontal gap, the hit finding efficiency must be calculated more carefully. In particular, because the height of the sensitive part of each muon scintillation counter is about $30 \mathrm{~cm}$ in $y$ and is not further segmented, triggered tracks can pass through the detector across a range of about $30 \mathrm{~cm}$ in $y$. Therefore, the $z$ location of the track's crossing point over the horizontal gap varied, leading to the track crossing varying numbers of collection plane wires in each of the stacked TPCs across many events. So the denominator of Equation 6.14 for each APA was not straightforward to calculate.

A method was devised to overcome these difficulties which attempts to determine the $z$ location of the horizontal gap crossing to allow a reasonable calculation of the number of expected hits in any given event, on average. This proceeded by first considering that the collection plane wires on each (bottom and top) APA are stacked, and therefore they read out the same $z$ coordinate but at different $y$ coordinates. So, the $y$ position of any given track is single-valued in $z$. That is, it makes no sense for a hit to exist on both of the two wires which share the same $z$ coordinate. Even further, hits must be on consecutive wires on each APA, with the track beginning in TPC 3 and ending in TPC 5, as wire number increases. These characteristics are illustrated by several example events in Table 6.3 where stacked wires are shown as single characters, with Xs denoting hits on the wire. In example (a), it is reasonable to assume that the track crosses the horizontal gap somewhere between wire numbers 21 and 50 , however, due to relatively low efficiency in this region, a more accurate determination of the crossing point is not possible. In general, the location of the gap crossing is determined as the midpoint between the last wire hit in TPC 3, and the first wire hit in TPC 5, to spread losses in efficiency equally between the two TPCs. Example (b) shows a much better case, where the crossing point is very clearly at the $z$ position of wire number 8. Examples (c) and (d) show situations where it is not possible to determine the crossing point, as the same logic cannot be applied and the efficiency is neither calculated nor included in future plots. If the horizontal gap crossing was determinable, the number of non-bad wires up to the gap crossing (for TPC 3 ) and the number of non-bad wires after the gap crossing (for TPC 5) was used in the denominator of Equation 6.14.

The number of hits found on each wire in each TPC is shown in Figure 6.22. For the data-taking runs described in Section 6.1.3, half of the TPC 1 collection plane wires were turned off, so no hits were found in that region. For TPCs 3 and 5, the slanting histogram 


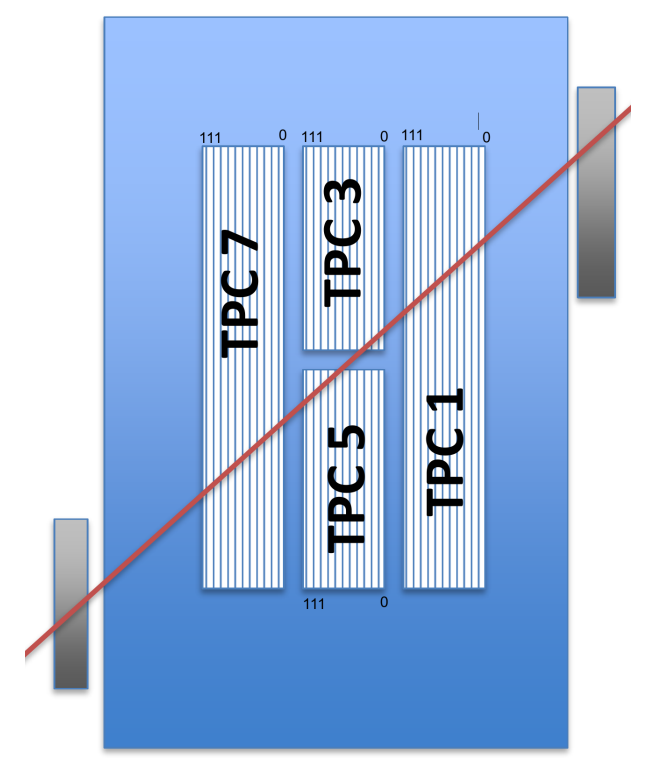

Fig. 6.21 Cartoon diagram of typical East-West muon event in the 35-ton detector. In this view, wire numbers (edges labelled) increase from right to left.

Table 6.3 Examples of reconstructed events which cross the horizontal gap between TPCs 3 and 5. "l" = Edge wire, " $"$ " = Bad wire, "X" = Hit found on wire, "." = Hit not found on wire.

\begin{tabular}{|c|c|c|}
\hline (a) & $\begin{array}{l}\text { TPC } 3 \\
\text { TPC } 5\end{array}$ & 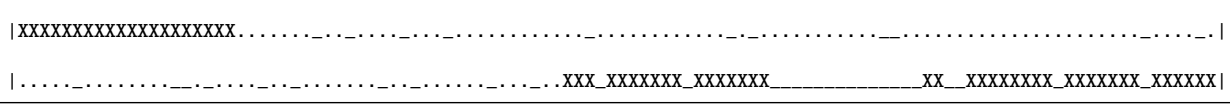 \\
\hline (b) & $\begin{array}{l}\text { TPC } 3 \\
\text { TPC } 5\end{array}$ & 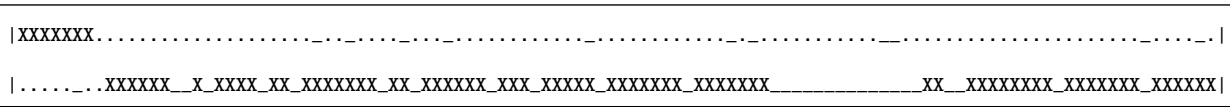 \\
\hline (c) & $\begin{array}{l}\text { TPC } 3 \\
\text { TPC } 5\end{array}$ & 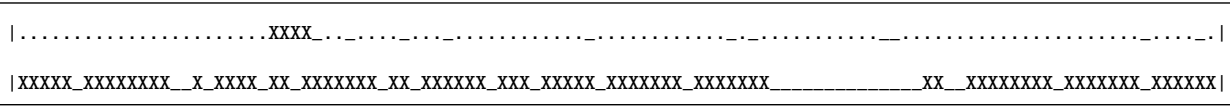 \\
\hline (d) & $\begin{array}{l}\text { TPC } 3 \\
\text { TPC } 5\end{array}$ & 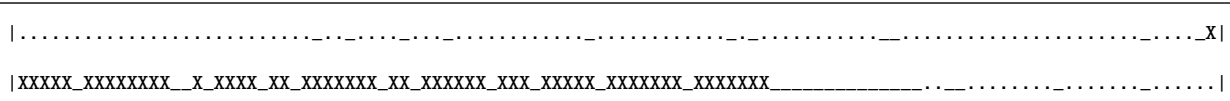 \\
\hline
\end{tabular}


is a reflection of the geometrical properties of the tracks crossing the horizontal gap. For example, in most cases, the track crossed halfway across the gap, depositing charge in the first half of TPC 3 and the last half of TPC 5 (stacked in the figure as in the real geometry). However, the variation in the $y$ coordinate of the tracks and the resulting variation in the $z$ coordinate of the gap crossing led to the slanted behaviour of the hit histograms.

For the full 35-ton data run analysed here, the hit finding efficiency is shown by wire number in each TPC in Figure 6.23. For most wires, the hit finding efficiency is higher than $80 \%$ which is consistent with the hit finding efficiency for simulation as in the blue curves in Figure 6.15. The phenomenon where the efficiency drops on wires near the edges of the APA is explained by the conservative nature of the THB in assuming the existence of non-"found" hits. As described in Section 5.3.4, a hit was "assumed" on a wire only if it had neighbouring wires on both sides which contain a reconstructed "found" hit. Therefore, the nearer a wire was to the edge of an APA, the less likely it was to have a wire with a "found" hit on both sides for interpolation of the hit location.

\subsection{Comparing Data With Simulation}

The combination of the track and hit finding efficiency and purity, as well as the charge resolution information is used to reduce the bias of the electron lifetime measurement further. Several significant sources of measurement bias, as well as two of the methods used to mitigate the effects of the bias, are described here.

\subsection{1 "Assumed" hits in data and simulation}

One comparison made between the reconstruction of data and simulation is the production of "assumed" hits by the RHF. These hits are not found because their amplitude is below the threshold, but their presence is assumed by neighbouring "found" hits, as described in Section 5.3.4. Between data and simulation, the fraction of all hits which are "assumed" should be equal on every wire, on average. Figure 6.24 shows that there are slightly more assumed hits in data than in simulation, meaning that each wire is reconstructed similarly, but not identically, in both data sets.

\subsubsection{Reducing bias by correcting signal scale factors}

While all aspects of the 35-ton electronic noise were directly reproduced in the simulation, uncertainty remains in the collection wire signal simulation. While the convolution of the induced signal current with the detector effects attempted to reproduce the signal in 

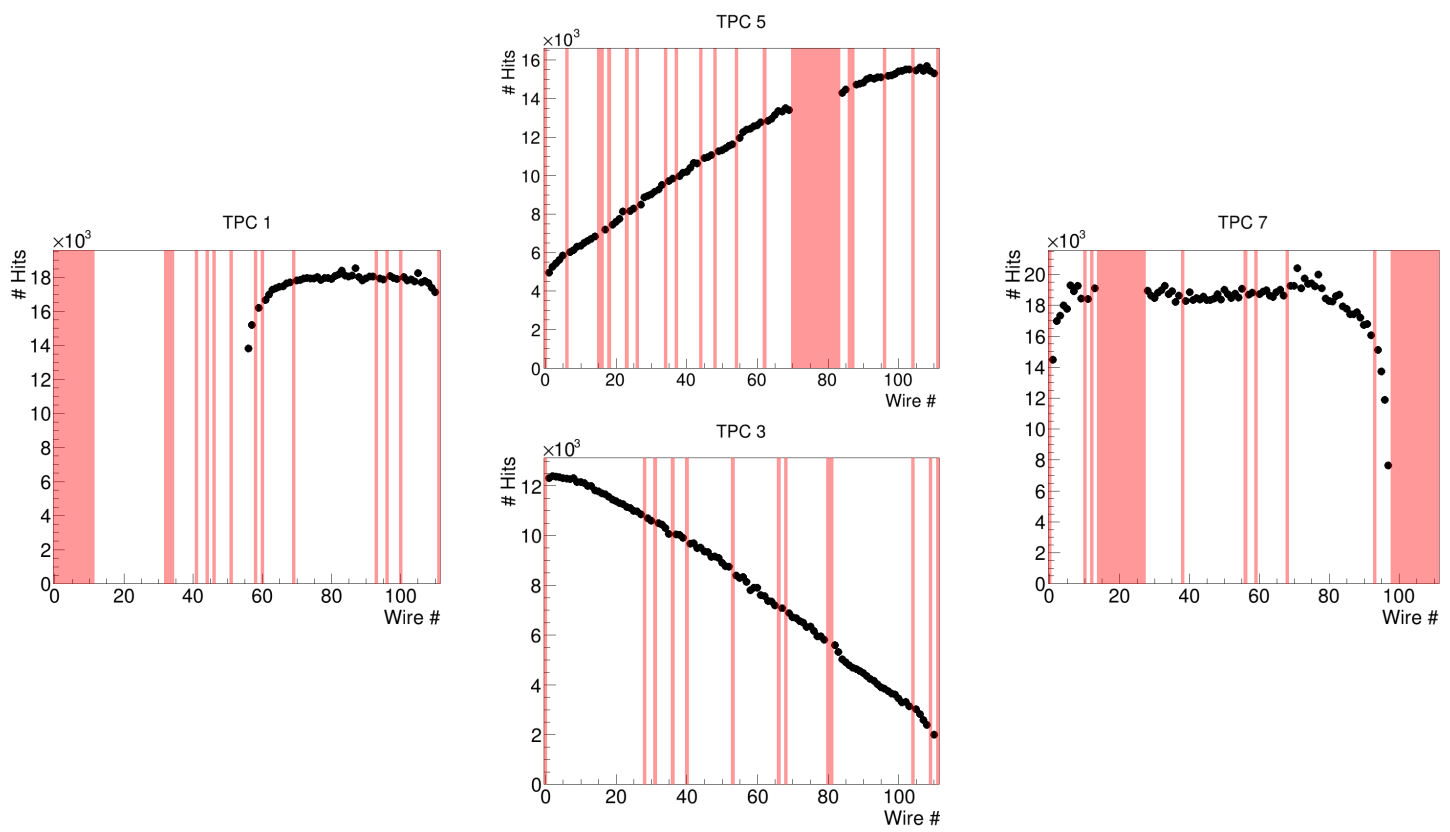

Fig. 6.22 Number of hits on each wire in each long-drift TPC. Red regions denote bad wires.
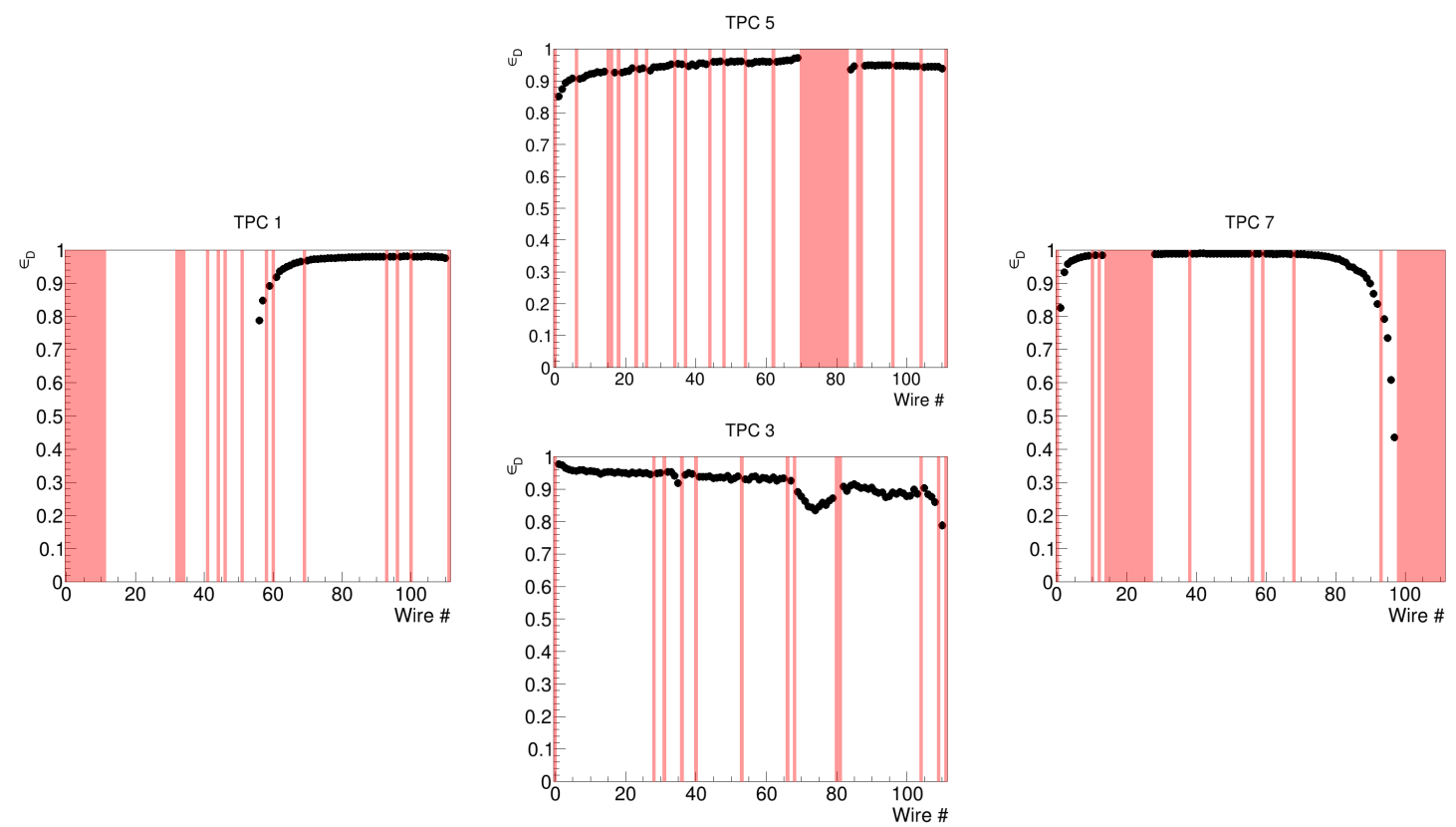

Fig. 6.23 Hit finding efficiency on each wire for each of the long-drift TPCs. Red regions denote bad wires. 


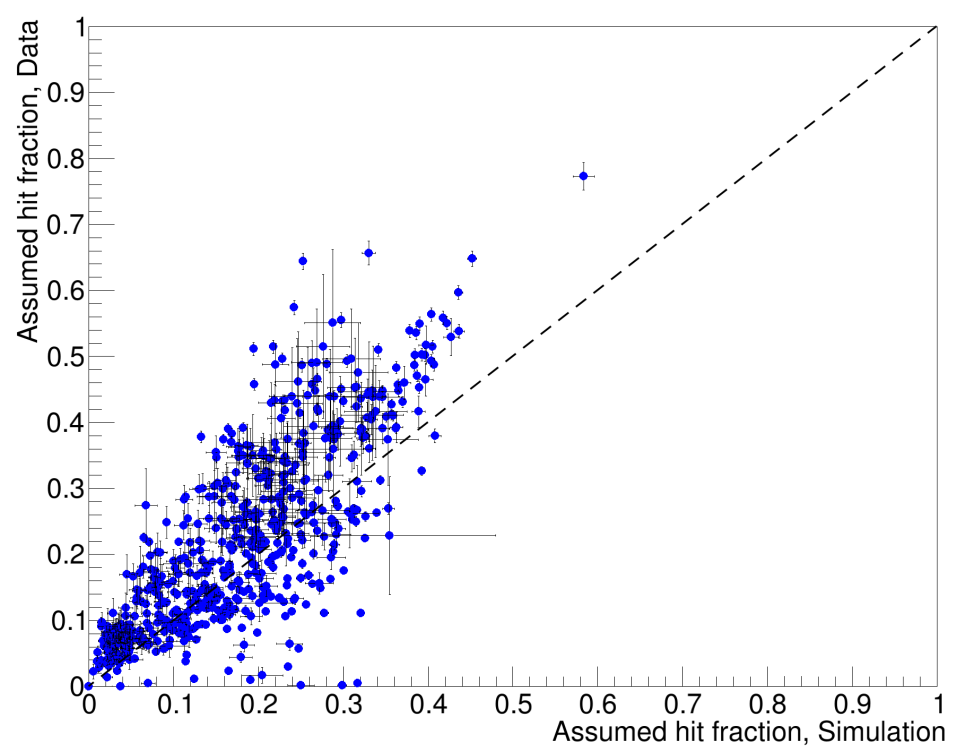

Fig. 6.24 "Assumed" hit fraction for data and simulation for every collection plane wire. The dashed line shows equal "assumed" hit fraction in data and simulation.

simulations accurately, there is evidence of additional factors which systematically influence the signal shaping in the 35-ton data. For example, differences in the actual amplification gain setting across wires, between ASICs, and over a period of time can cause offsets in the calculation of the total reconstructed hit charge on that wire. Additional effects, such as nonlinearities in digitisation or differences in pulse shaping characteristics due to electronics noise affect the most probable hit charge on a particular wire, over the course of the data run.

The intercept parameter in the exponential fit (Equation 6.1), $(d Q / d x)_{0}$, gives the most probable charge deposited in the TPC in the absence of charge attenuation due to impurities. Comparison of 35-ton data $\left((d Q / d x)_{0}=2433 \mathrm{ADC} / \mathrm{cm}\right)$ and the simulated data with $\mu=1$ $\left((d Q / d x)_{0}=2099 \mathrm{ADC} / \mathrm{cm}\right)$ shows that the simulation did not accurately model the true $\mathrm{S} / \mathrm{N}$ of 35 -ton data. Figure 6.25 shows that scaling up the amplitude of signal simulation by an additional $15.3 \%$ more closely reproduced the 35 -ton data. This bias was mitigated by using the data sets with $\mu=1.2$ in comparing the simulation with real data. The remaining differences between simulation and 35-ton data were included in the overall estimation of systematic uncertainty.

\subsubsection{Data, Simulation, and MC Truth hit charge}

It is useful to compare real data to simulated data, but also simulated data to MCT information, over the full drift length. By plotting the three $d Q / d x$ distributions on the same $x$ scale, any 


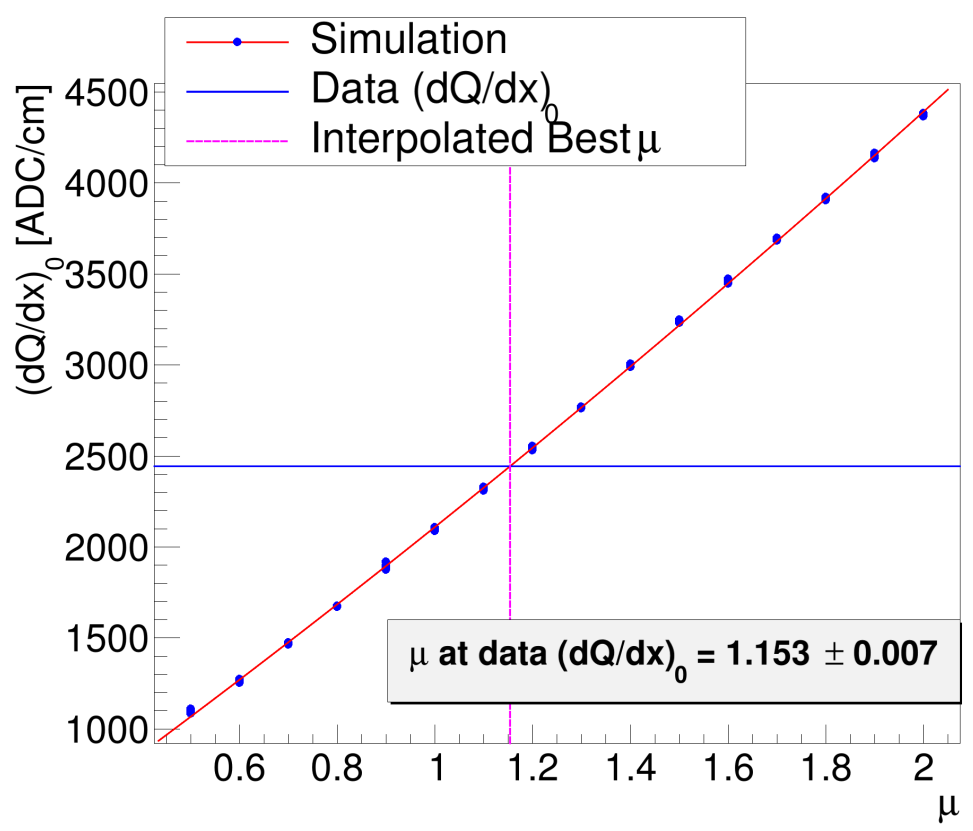

Fig. 6.25 Exponential fit $(d Q / d x)_{0}$ parameter for all simulated datasets. Vertical blue line represents the true data $(d Q / d x)_{0}$ value, and the true $\mu$ parameter is determined through interpolation.

differences between the sources of data should be noticeable. The plots in Figure 6.26 show hit $d Q / d x$ for four different drift distance bins, (a) near the anode, (b) $1 / 3$ of the full drift, (c) $2 / 3$ of the full drift, and (d) near the cathode. The similar distribution shapes of data and simulation reflect the effects of accurate modelling of the detector noise in the simulation. Any shifts observed in the data and simulation MPVs across the full drift distance would be representative of an unrealistically simulated electron lifetime. The roughly equal MPV $d Q / d x$ for data, simulation, and MCT for short and long drift distances shows the correct scaling factor of signals in the simulation and hints at a correct electron lifetime of $4 \mathrm{~ms}$.

In Figure 6.26, the 35-ton simulation charge distributions are slightly narrower than the 35-ton data charge distributions, the discrepancy becoming less significant for longer drift distances. This phenomenon summarises the remaining factors which prevent accurate simulation of 35-ton noise and signal characteristics, including positive space charge effects which are not simulated and inaccurate simulation of 35-ton signal shapes. Further discussion of these and other systematic uncertainties is in Section 6.5.1. The enhancement of MCT hit charge at low $d Q / d x$ values in Figure 6.26 is thought to be attributed to incorrectly summed hit charges in the simulation, for example, if one true hit is incorrectly split by the reconstruction into two separate hits with smaller individual charges. 


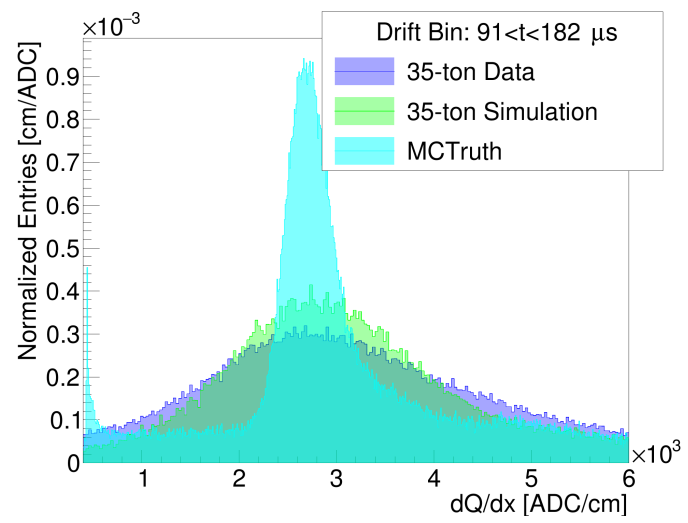

(a)

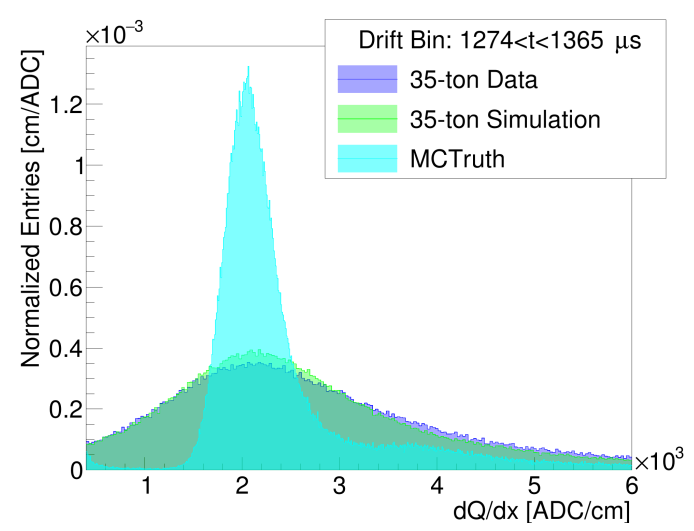

(c)

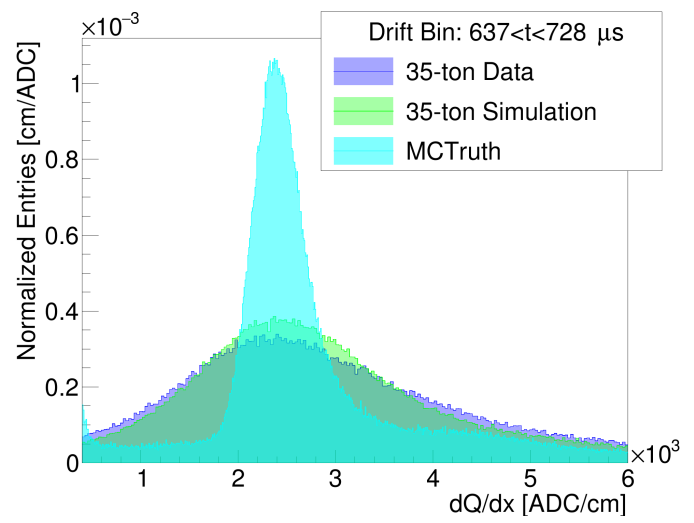

(b)

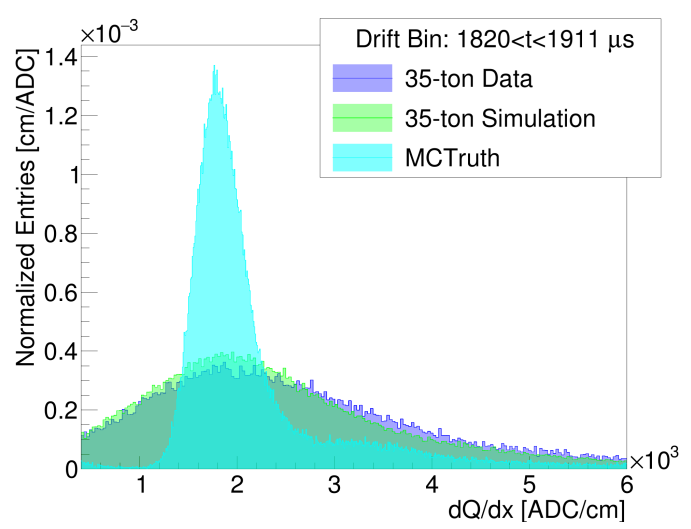

(d)

Fig. 6.26 Data, simulation, and MCT $d Q / d x$ histograms for (a) short, (b) short-middle, (c) middle-long, and (d) long drift distances. Simulated samples shown use an electron lifetime of $4 \mathrm{~ms}$. 


\subsubsection{Impact of hit finding threshold}

Another significant source of bias in the analysis of noisy data from the 35-ton is the thresholding problem, which may explain the difference in data (or simulation) and MCT most probable $d Q / d x$ from the previous section. Figure 6.27 illustrates the effect of the variable threshold used in the RHF. Because the RHF threshold depends on the noise level of the particular wire signal and varies across wires, the threshold function is not a simple step function. Instead, it resembles a sigmoid shape, as observed by the distribution of the hit finding efficiency versus charge in Figure 6.15. Then, when the charge is attenuated, and the Landau encroaches on the threshold, the resulting distribution is a convolution of the Landau and the threshold function, and the maximum function value becomes further from the real Landau MPV.

In terms of the electron lifetime analysis, and indeed any calorimetric measurement, this thresholding effect has significant consequences. First of all, as the drift distance increased the Landau MPV fell exponentially, as expected. However, once the charge was sufficiently attenuated with respect to the threshold function, the Landau MPV appeared to level off, invalidating the exponential decay assumption which is essential to the lifetime analysis. Figure 6.6 indeed shows that the binned Landau MPVs did not decay purely exponentially over the full drift length. The more distant drift bins consistently have a higher most probable hit charge which artificially increased the measured lifetime. This positive bias in the measured MPV was one of the primary sources of error in the electron lifetime analysis and motivated the need for some fiducial or hit charge cut.

The hit finding efficiency plot in Figure 6.15 suggests the sigmoid cutoff may be around $500 \mathrm{ADC} / \mathrm{cm}$. At worst, it is possible that the effects of the threshold were much more significant than the illustrations in Figure 6.27 suggest, and none of the measured Landau MPV values in this analysis represent the true MPVs from the underlying physical processes.

\subsubsection{Reducing bias by applying fiducial cuts}

The bias between the true MPV and the observed MPV increases with drift distance, which motivates a fiducial cut for the lifetime measurement. Defining a threshold for this cut, however, is not possible without prior knowledge of the true lifetime. Nevertheless, it is known that there is less bias in the measured hit charges with shorter drift times (near the anode) than with longer drift times (near the cathode). Based on this observation, an arbitrary fiducial cut of $1 / 2$ the full drift time was imposed to reduce the bias on the electron lifetime measurement. Stricter cuts are hypothesised to reduce the bias even further, but this dependence and its effects on overall measurement uncertainties are not studied. To illustrate 

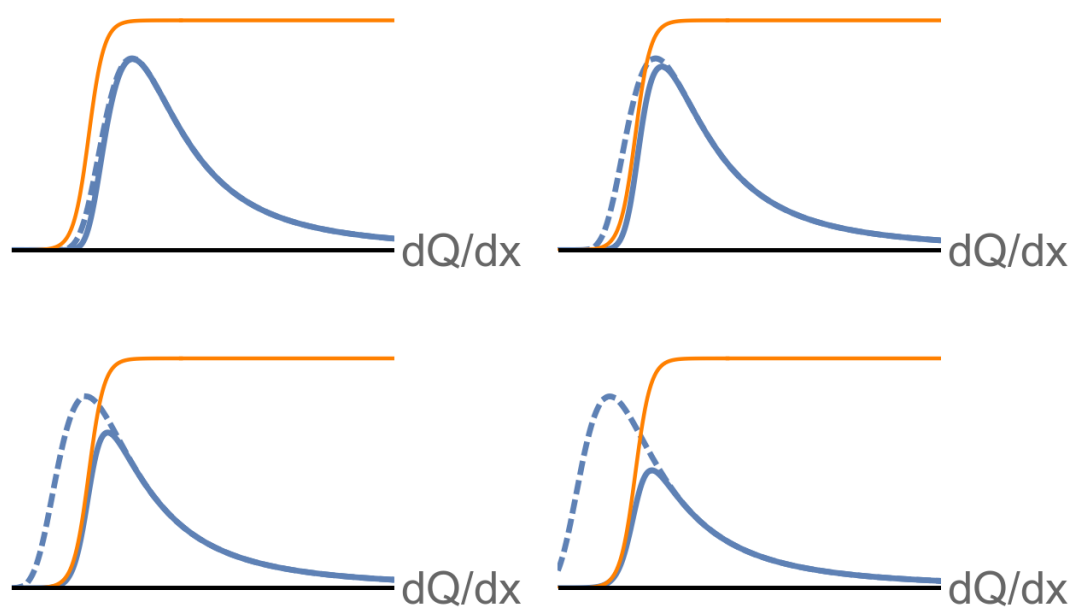

Fig. 6.27 Hypothetical effect of variable hit finding threshold (orange) on Landau (blue) MPV measurement. The dashed curve represents the parts of the Landau which are cut off by the threshold.

the effectiveness of the $50 \%$ cut, the measured lifetime from the full detector drift distance is observed as $4.73 \mathrm{~ms}$, compared to $4.24 \mathrm{~ms}$ with the 5-50\% cut imposed (see Figure 6.6). A drift-distance-dependent bias clearly exists, and is attributed to the decreasing hit efficiency and worsening charge resolution at longer drift lengths. The bias is quantified by comparing the lifetimes extracted from the simulated data sets to the true simulated lifetimes.

\subsection{Correcting for Electron Lifetime Bias}

By analysing the electron lifetime of many simulated samples of varying parameters and comparing the results with the measured data, the lifetime of the 35-ton data set was extracted. Figure 6.28 shows the observed raw lifetimes, as a function of noise level, separated by the true simulated lifetime. For simulated samples, the horizontal scale was $m=\mu / 1.153$, correcting for signal simulation error (Section 6.4.2). The bias in the observed lifetime for higher noise can be seen for lower $\mu$ values, a result of the drift dependent upward bias in Landau MPVs (Section 6.4.4). The measured lifetime for the 35-ton data is consistent with the simulated samples with $4.0 \mathrm{~ms}$ lifetime, and $\mu$ of 1.1 and 1.2 , confirmed by the similarities shown in Figure 6.29. The corrected electron lifetime of 35-ton data was more precisely determined through interpolation.

To predict the corrected data electron lifetime based on the observed raw electron lifetime and the detector noise level, a multivariate linear regression was performed on the simulated 


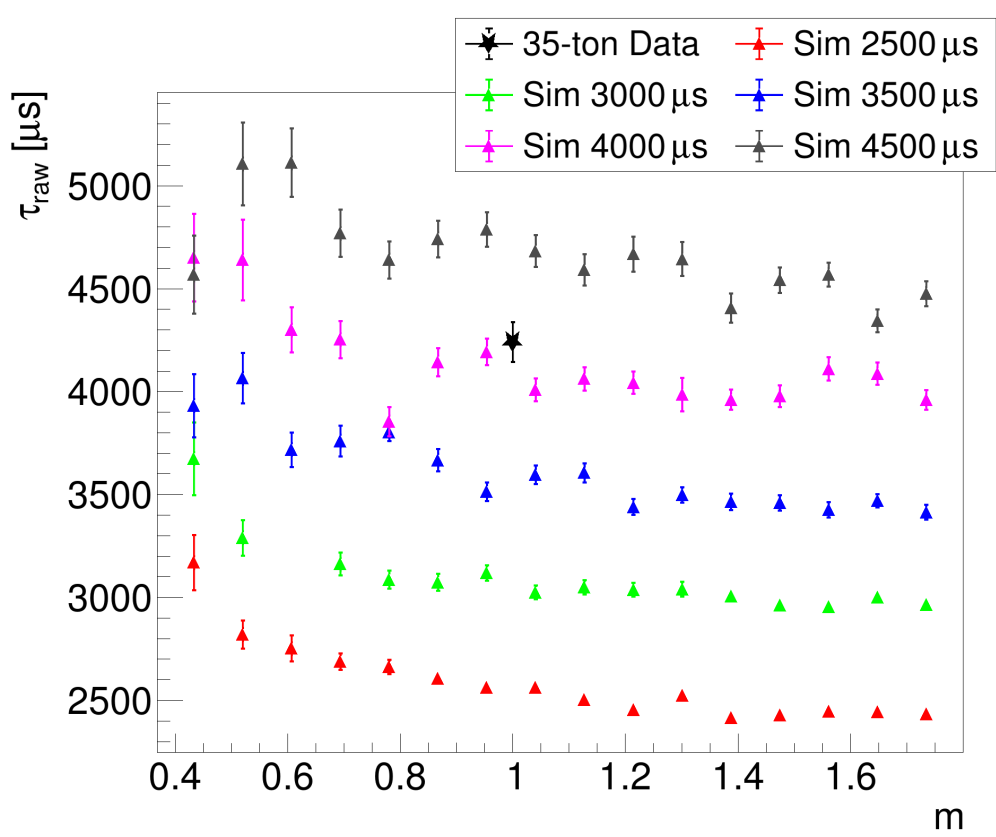

Fig. 6.28 Measured electron lifetime of simulated samples and 35-ton data for different values of $m=\mu / 1.153$.

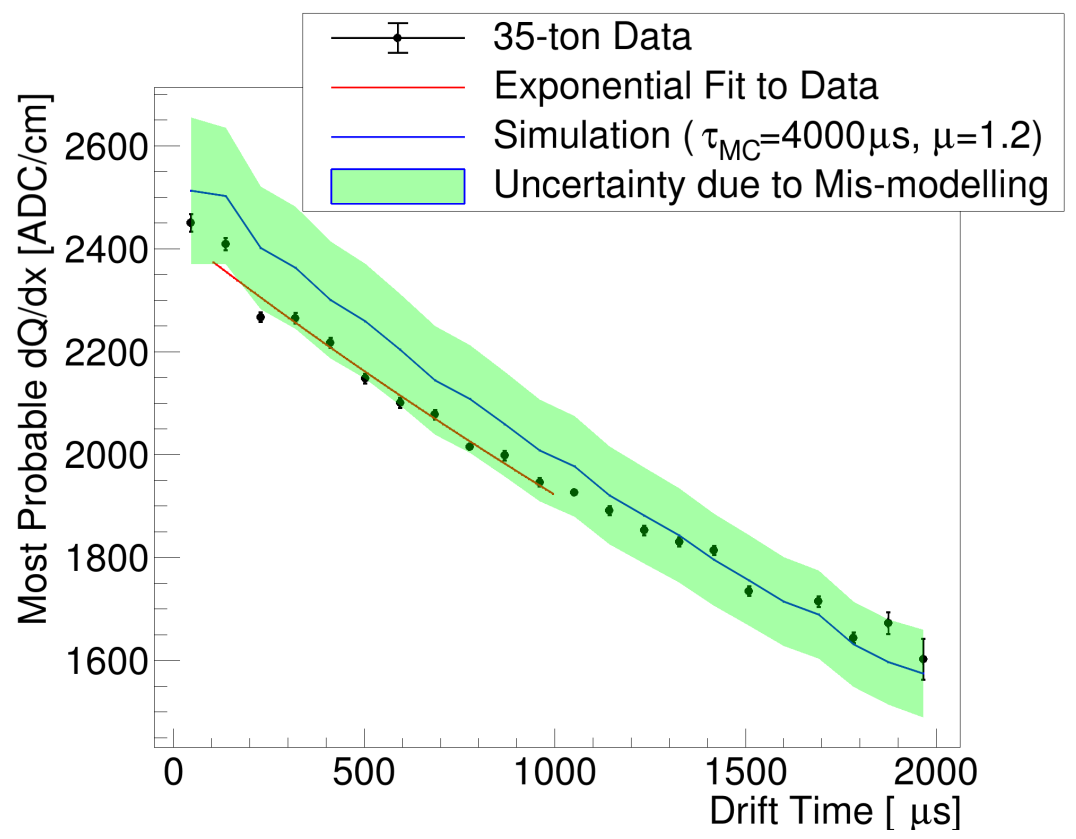

Fig. 6.29 Data $d Q / d x$ MPV (black) overlaid with most similar simulated $d Q / d x$ MPV (blue). Green band represents the uncertainty due to $\mu$. 
sample results. The linear model

$$
\vec{\tau}_{\mathrm{MC}}=\mathbf{X} \vec{\alpha}
$$

was investigated where

$$
\mathbf{X}=\left(\begin{array}{cccccc}
1 & m_{1} & \tau_{\text {raw }, 1} & m_{1} \tau_{\text {raw }, 1} & m_{1}^{2} & \tau_{\text {raw }, 1}^{2} \\
& & & \vdots & & \\
1 & m_{n} & \tau_{\text {raw }, n} & m_{n} \tau_{\text {raw }, n} & m_{n}^{2} & \tau_{\text {raw }, n}^{2}
\end{array}\right)
$$

for $i=1 \ldots n \mathrm{MC}$ samples and

$$
\vec{\alpha}=\left(\alpha_{1} \cdots \alpha_{6}\right)^{T} .
$$

$\vec{\tau}_{\text {MC }}$ is the vector of true simulated electron lifetimes for each of the $n$ samples, $\tau_{\text {raw }, i}$ is the observed raw electron lifetime for sample $i$, and $m_{i}=\mu_{i} / 1.153$. The $\vec{\alpha}$ coefficients were calculated as

$$
\vec{\alpha}=\left(\mathbf{X}^{T} \mathbf{X}\right)^{-1} \mathbf{X}^{T} \vec{\tau}_{\mathrm{MC}}
$$

using the results from all $n=80 \mathrm{MC}$ samples.

The corrected data electron lifetime was then calculated as $\tau_{\text {corr }}=\mathbf{X}_{\text {data }} \vec{\alpha}$ where $\mathbf{X}_{\text {data }}$ is the row vector determined from Equation 6.16 with $n=1, m_{1}=1.0$, the data observed lifetime taken from Figure $6.6\left(\tau_{\text {raw }, 1}=4.24 \mathrm{~ms}\right)$, and $\vec{\alpha}$ determined from Equation 6.18. This process revealed the corrected lifetime for 35 -ton data to be $\tau_{\text {corr }}=4.12 \mathrm{~ms}$.

To calculate the error on this prediction, we use the classical errors-in-variables method [178] to take into account uncertainties in $m_{i}$ and $\tau_{\text {raw }, i}$. In this method, the errors of the regression model combine the statistical fluctuations and the measurement uncertainties,

$$
\vec{\tau}_{\mathrm{MC}}=(\mathbf{X}-\mathbf{u}) \vec{\alpha}+\vec{\epsilon}=\mathbf{X} \vec{\alpha}+(\vec{\epsilon}-\mathbf{u} \vec{\alpha})
$$

where $\vec{\epsilon}$ is the vector of residuals and $\mathbf{u}$ is a matrix which contains the errors on the model variables in $\mathbf{X}$ for all MC samples. For the simulated samples, the measurement uncertainty on $m_{i}$ is chosen arbitraily to be 0.05 to account for signal mis-modelling in the simulation and the uncertainty on $\tau_{\text {raw }, i}$ is the error on the exponential fit lifetime parameter. The mean squared error of the regression is an estimate of the variance and is calculated as [179]

$$
\mathrm{MSE}=\frac{\vec{\epsilon} \cdot \vec{\epsilon}}{n-p}=\frac{\left(\vec{\tau}_{\mathrm{MC}}+\mathbf{u} \vec{\alpha}-\mathbf{X} \vec{\alpha}\right)^{T}\left(\vec{\tau}_{\mathrm{MC}}+\mathbf{u} \vec{\alpha}-\mathbf{X} \vec{\alpha}\right)}{n-p}
$$


for $p=6$ model variables using the best-fit parameters determined in Equation 6.18. The standard error of the predicted corrected data lifetime is [179]

$$
\text { s.e. }=\sqrt{\operatorname{MSE}\left(\mathbf{X}_{\text {data }}\left(\mathbf{X}^{T} \mathbf{X}\right)^{-1} \mathbf{X}_{\text {data }}^{T}\right)}
$$

and the $1 \sigma$ prediction interval for the corrected electron lifetime is

$$
\tau_{\text {corr }} \pm \sqrt{\mathrm{MSE}+[\text { s.e. }]^{2}}=0.134 \mathrm{~ms}
$$

This interval is then taken as the uncertainty associated with the interpolation of the data electron lifetime due to measurement errors in the simulated samples. The measurement uncertainty of the data raw lifetime exponential fit parameter $(0.097 \mathrm{~ms}$, as in Figure 6.6) and the interpolation error above are combined in quadrature to give the total statistical uncertainty in the corrected data electron lifetime measurement as $0.165 \mathrm{~ms}$.

\subsubsection{Systematic Uncertainties}

The systematic uncertainty associated with the biases introduced by the noise was taken as the magnitude of the bias shift in the lifetime calculated in the previous section, $4.24-4.12=$ $0.12 \mathrm{~ms}, 2.8 \%$. The magnitude of this uncertainty is assumed to account for the effects of low hit finding efficiency on the true Landau MPV, and the poor charge resolution for the relevant region of hit charge, both caused by the high level of noise in the detector.

Further uncertainty may be attributed to the accumulation of positive space charge in the TPC, which, because of their low mobility in comparison to the negative drift electrons, distorted the electric field [91]. Based on the nominal drift field of $E_{0}=250 \mathrm{~V} / \mathrm{cm}$, the full drift distance of $2.2 \mathrm{~m}$, and the rate of creation of positive ion density due to cosmic ray muons at the surface of the earth (about $1.8 \times 10^{-10} \mathrm{C} / \mathrm{m}^{3} / \mathrm{s}$ ), the actual electric field becomes $0.89 \mathrm{E}_{0}$ at the anode, and $1.19 \mathrm{E}_{0}$ at the cathode. This overall field non-uniformity affects the probability of a drift electron to recombine with an argon ion. According to [101], the fraction of ionisation electrons from a $1.8 \mathrm{MeV}$ muon which survives recombination is $64.3 \%$ in the absence of space charge, and $62.5 \%$ at the anode and $66.7 \%$ at the cathode with space charge distortions. This means a $4.2 \%$ difference in available charge for drifting between the anode and cathode as a result of recombination alone. To determine the effect such a difference has on the decay lifetime we consider two exponential functions, $f(t)=A e^{-t / \tau}$ and $g(t)=A e^{-t / \tau^{\prime}}$, which are equal valued at $t=0, f(0)=g(0)$, and differ by a factor of $\alpha$ at $t=T, f(T)=(1+\alpha) g(T)$. Therefore, the lifetime parameter adjusted for space charge, $\tau^{\prime}$, as 
a function of $\alpha$, is

$$
\tau^{\prime}(\alpha)=\frac{T \tau}{T+\tau \log (1+\alpha)} .
$$

Thus, for this analysis ( $\tau=4.12 \mathrm{~ms}, T \approx 2 \mathrm{~ms}$, and $\alpha=0.042$ ), the expected contribution of space charge distortions in electric field to the total systematic uncertainty of the electron lifetime to be $7.8 \%$ for the fiducial region described in Section 6.4.5.

Remaining differences between data and simulation contribute to the overall systematic uncertainty in the lifetime measurement. These sources of error include the effects of transverse diffusion, channel-by-channel gain variations, and signal simulation errors in the field and electronics response functions. While these sources of error have not been studied in detail, they are expected to contribute roughly $5 \%$ error based on the reported results of other similar LAr TPC experiments [180].

Other systematic uncertainties in this measurement may be due to the inefficient mixing of impurities throughout the full cryostat volume. Previous internal computational fluid dynamics studies of the impurity mixing in the cryostat, taking into account fluid flow through the TPC field cage, suggest the possibility of at least a $10 \%$ difference in the local LAr electron lifetimes between the location of PrM2 and within the TPC field cage [181]. This uncertainty in the local differences in impurity concentration was not incorporated into the overall systematic uncertainty but is left to the reader to consider when comparing the results of this analysis with the measured values from PrM2.

Therefore, the individually identified systematic uncertainties for the current lifetime measurement were combined in quadrature to give an overall uncertainty of $9.7 \%$, or $0.40 \mathrm{~ms}$. Finally, the measured lifetime of $\tau=4.12 \pm 0.17$ (stat.) \pm 0.40 (syst.) $\mathrm{ms}$ is marginally consistent with the average of the purity monitor measurements, $2.8 \pm 0.1$ (stat.) \pm 0.5 (syst.) ms, for the same dataset.

\subsection{Summary}

Despite the biases which influence the 35-ton data as a result of many various factors exacerbated by high electronic noise, a measurement of the electron lifetime was still made. Moreover, the measured value is marginally consistent with the purity monitors' measurement over the same data taking time span.

Any LAr TPC experiment must contend with some level of electronic noise. The actual $\mathrm{S} / \mathrm{N}$ requirements are defined for the noise to be guaranteed not to influence the physics ability of the detector. The general Deep Underground Neutrino Experiment (DUNE) requirement is 9:1. Even though this was not achieved in the 35-ton experiment, it was still possible to make 
a simple calorimetric measurement, albeit with substantial uncertainty and significant biases. For high-precision physics measurements, these uncertainties in reconstruction efficiency and charge resolution would inevitably dominate any other source and would render the measurement useless. A measurement so comparatively low level as the electron lifetime was still completed to within roughly the same accuracy as other methods of measuring the same value, namely dedicated PrMs.

As the electron lifetimes in LAr experiments increase with advances in purification and cryostat technologies (experiments have shown lifetimes greater than $20 \mathrm{~ms}$ [142]), more precise measurements are required, and the noise level requirements become more and more strict. It is likely that the methods described here will be not sufficient, assuming the same noise level as the 35-ton. If the 35-ton noise were any worse, this measurement would not be possible because all real signals in the detector would be overshadowed by the noise, preventing observation of minimally ionising particle events. If the 35-ton noise were any better, these methods would not be necessary as it would be possible to accurately deconvolve detector, electronics, and noise effects from the true ionisation electron signals. 


\section{Chapter 7}

\section{The Sheffield LAr Test Stand}

The rise in importance of liquid argon (LAr) time projection chambers (TPC) for high energy particle physics motivates more precise and thorough measurements of the fundamental electron transport and light production properties of LAr than previously reported in Chapter 2. In particular, longitudinal diffusion, space charge, and electron multiplication due to secondary ionisation are areas in which additional research would be welcomed. Few studies of these phenomena, in particular, have been performed under the experimental conditions similar to what is and will be used in the next generation of massive LAr TPCs, i.e. low impurity concentrations and small electric fields. Thus, it is vitally important to understand the effects these processes might have on the generated signals in such detectors to better understand the detector response and reduce systematic uncertainties. For example, MicroBooNE is currently studying the effects of space charge [182] and how it affects basic physics measurements such as the drift electron lifetime [180]. Also, the current best measurements of transverse diffusion in LAr have only recently been published [65], which still leaves room for better analyses and validation studies.

In addition to studying the properties of LAr relevant to the operation of massive LAr TPCs for neutrino or dark matter physics, there is significant interest in using noble liquids for medical physics applications (for example, [183]). Positron-emission tomography (PET), in particular, can be massively improved by the use of better scintillating materials with greater spatial and temporal resolution while maintaining a similar light yield, which noble liquids can provide over the previous generation of solid state crystals. Additionally, with the recent rise in popularity of thick gaseous electron multipliers (THGEM) for particle detection [184], there is a growing interest in using THGEMs in a noble liquid to take advantage of the excellent charge and scintillation properties (see, for example, [185]).

Given the strong current interest in LAr across a wide range of disciplines, there is a clear motivation to construct and operate a general and adaptable high purity LAr test facility. In 
2013, construction of such a test stand at Sheffield began, the design of which was based on a similar facility at Brookhaven National Laboratory [186, 187].

In this chapter, the design and construction of the test stand are introduced, including the physical cryogenic system components, the thermodynamics governing the stable operation, and the electronics system for monitoring and controlling the system. Also, the results from an initial run of the test stand are presented, with argon recirculation and nitrogen cooling.

The author contributed to much of the work presented in this chapter. Specifically, the author organised and laid out the system for construction, selected and purchased nearly all of the instrumentation and slow control hardware based on physics requirements, and operated the system along with the other lab personnel.

\subsection{Design and Construction}

The Sheffield LAr test stand is a closed, recirculating system with the purification of oxygen and water impurities in the gas phase. The purifier is fully regenerable, in case it becomes saturated with impurities, therefore avoiding the need to replace filter materials. The heat flow from the surroundings into the LAr vessel is balanced by the cooling power of pressurised liquid nitrogen $\left(\mathrm{LN}_{2}\right)$. A schematic of the system's primary components is shown in Figure 7.1 and an image of the assembled system is shown in Figure 7.2.

\subsubsection{Cryogenics and Piping}

All components of the cryogenic system are constructed using stainless steel (SS) of various grades, including 304 and 316, depending on the part manufacturer. The LAr vessel is a double walled vacuum-jacketed SS Dewar (Cryofab part no. CF-9524-F) which minimises heat loss due to conduction and convection and is shown in Figure 7.3. The vessel has an internal diameter of 9.46 inches and depth of 24 inches, making a total interior volume of 27.6 L. The Dewar lid consists of a solid 12 in Conflat SS flange, with 5 SS baffles mounted $1 \mathrm{~cm}$ apart underneath the flange which significantly reduces argon vapour convective heat loss through the lid. Various perforations are made in the baffles to allow pipes, sensors, and cables to pass through to the experiment in the main Dewar volume. There is an outlet pipe on the bottom of the Dewar, used both for filling with LAr and for measuring the hydrostatic pressure at the bottom of the Dewar.

In normal operation, boiloff argon gas travels upwards through an opening in the lid, through a vacuum-jacketed pipe, and enters a 2 inch diameter and 13 inch long cylinder containing materials to purify the gaseous argon (see Figure 7.4). One third of the cylinder 
1. LAr temperature sensor

2. LAr capacitive level meter

3. LAr pressure transducer

4. $\mathrm{LN}_{2}$ temperature sensor

5. $\mathrm{LN}_{2}$ capacitive level meter

6. $\mathrm{LN}_{2}$ pressure transducer

7. Detector (Gaseous Photomultiplier)

8. $355 \mathrm{~nm}$ LED

9. Solenoid valve

10. Back pressure regulator

11. 12-pin HV electrical feedthrough

12. Penning gas inlet and outlet

13. Differential pressure meter

14. Ceramic heating tape

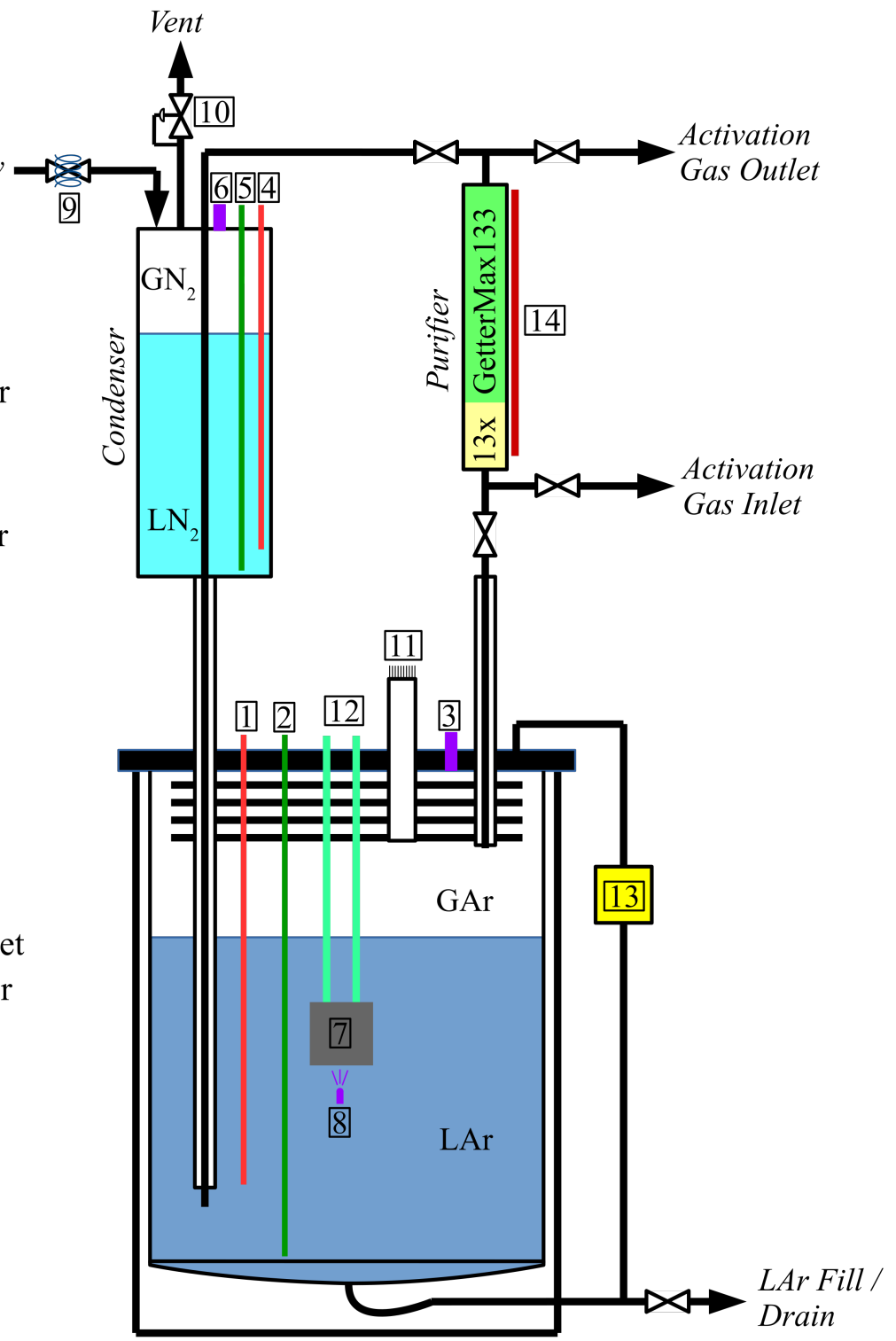

Fig. 7.1 Schematic of Sheffield LAr test stand and main operational components. 


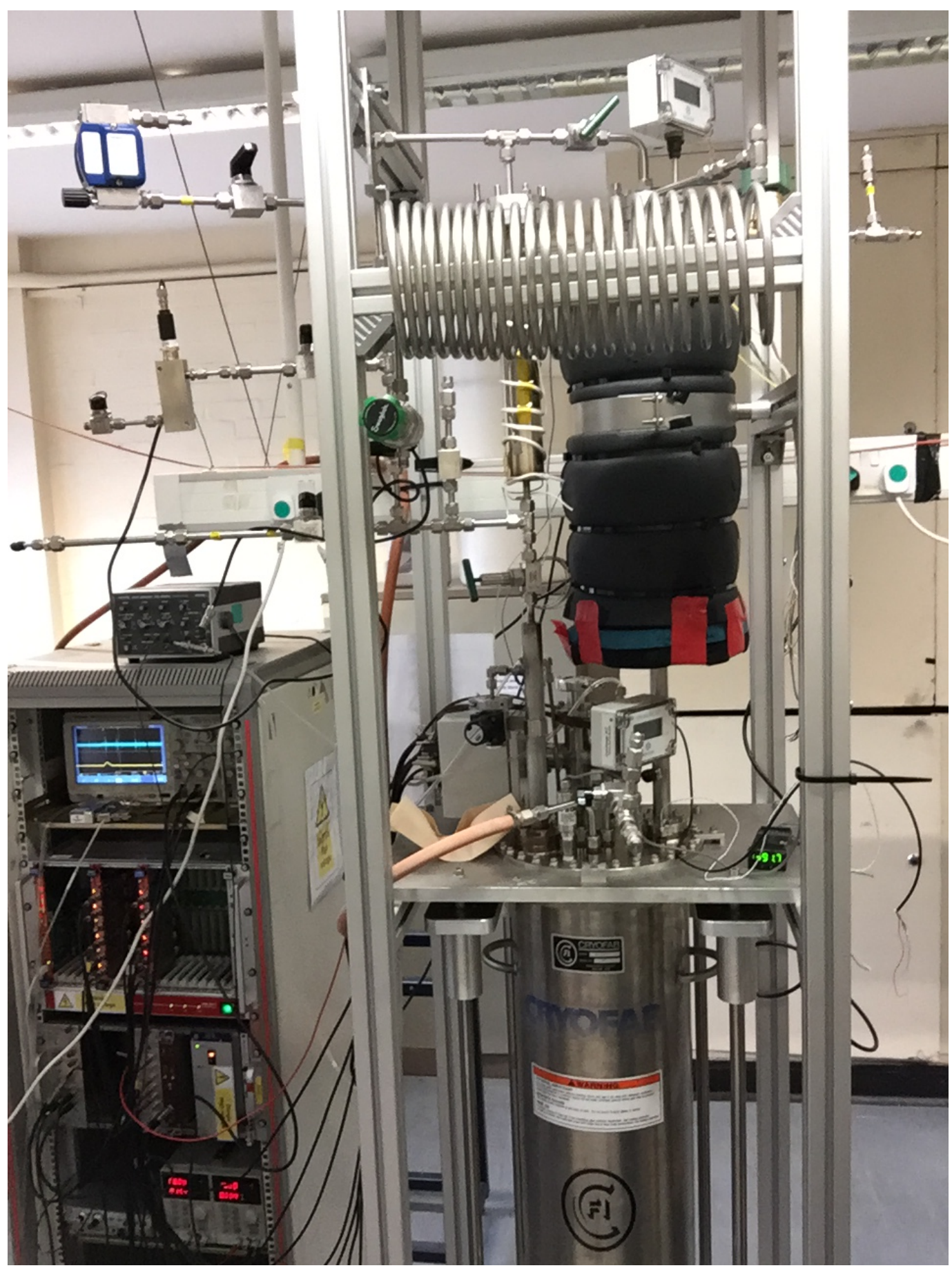

Fig. 7.2 Image of assembled LAr test stand and components. 


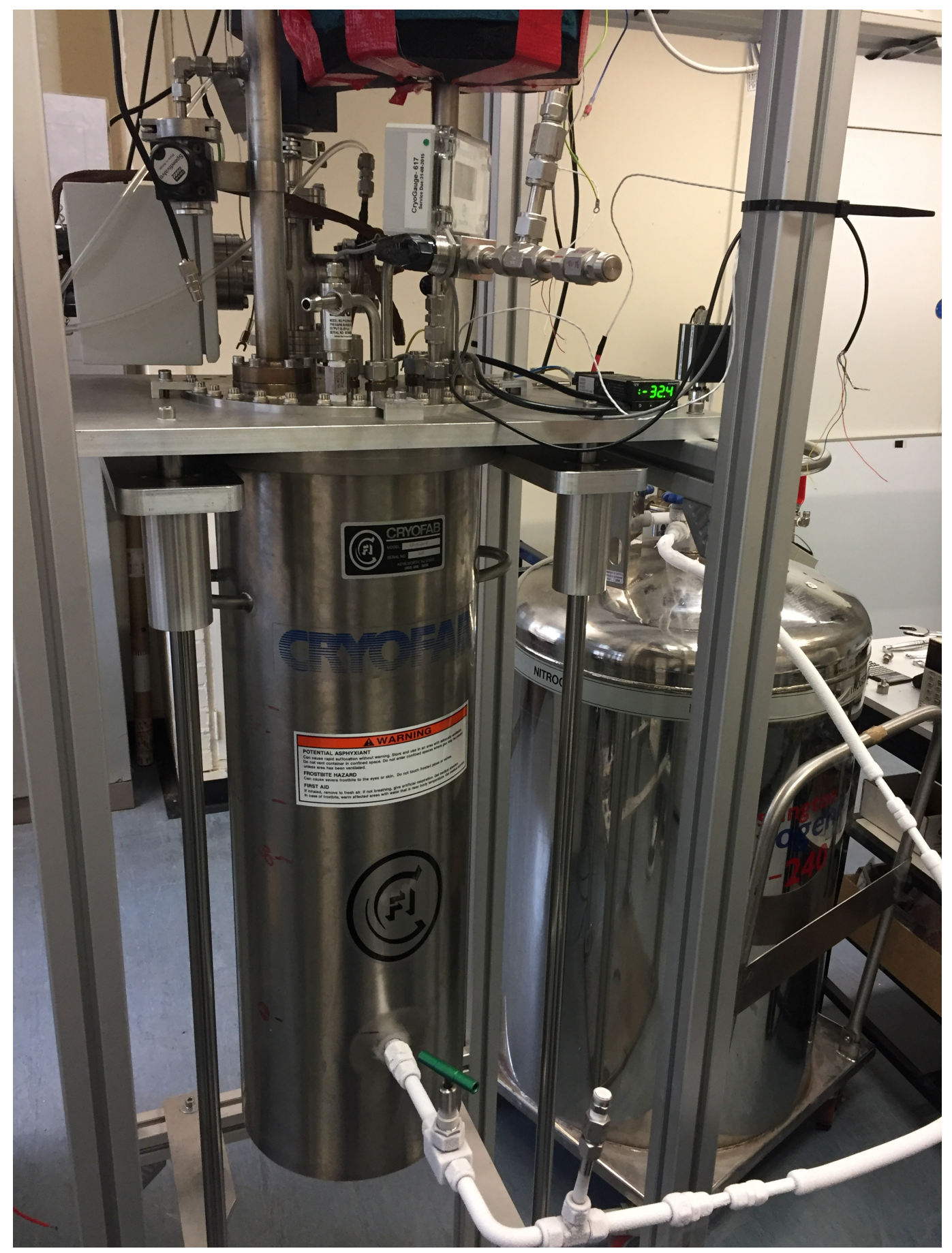

Fig. 7.3 Outside of main LAr Dewar during filling. 
volume is filled with 13x molecular sieve (Research Catalysts, Inc., RCI-DRI 13x, 8-14 mesh beads) for adsorbing $\mathrm{H}_{2} \mathrm{O}$, and the rest with a $\mathrm{Cu} / \mathrm{Zn} / \mathrm{Al}$ catalyst (Research Catalysts, Inc., GetterMax 133 copper catalyst, $3 \times 3 \mathrm{~mm}$ tablets) for chemically absorbing $\mathrm{O}_{2}$ impurities via oxidation. The oxygen getter is initially in an oxidised form, so must be activated (deoxidised) with hydrogen before purification can happen.

The purified gas then passes upwards through the top of the cylinder, around the top bend, and enters the recondensation cylinder volume (see Figure 7.5). The recondenser consists of an inner argon tube ( 2 in diameter), and an outer jacket isolated from the argon stream which contains $\mathrm{LN}_{2}$. The outer jacket has diameter 6 in and length $18.5 \mathrm{in}$, so contains about 7.6 $\mathrm{L}$ of $\mathrm{LN}_{2}$. The nitrogen jacket is pressurised to raise the temperature above the freezing point of argon. Condensation of the pure argon vapour occurs by convective and conductive cooling provided by the nitrogen, the liquid droplets fall down the pipe under the influence of gravity and are reintroduced into the bulk LAr near the bottom of the liquid volume.

Equilibrium in the argon system is achieved through the balancing of heat transfer in and out of the system. Heat transfers from the argon vapour to the $\mathrm{LN}_{2}$, causing condensation of the argon and vaporisation of the nitrogen. Because the argon system is closed, condensation reduces the pressure and temperature. Heat transferred into the system by the imperfect thermal insulation to the exterior causes vaporisation of the argon, increasing the temperature and pressure of the system. The heat transferred from the external environment into the argon system is precisely balanced with the heat transferred from the argon to the $\mathrm{LN}_{2}$ during recondensing. The heat transfer into the nitrogen from the argon, and indeed from the external environment, causes vaporisation, increasing the temperature and pressure in the $\mathrm{LN}_{2}$ vessel. The increase in pressure is balanced by the releasing of gas from the back pressure regulator, reducing the mass of $\mathrm{LN}_{2}$ in the system and the temperature. As the nitrogen mass decreases over the course of running, it is continuously refilled from an external supply.

The thermodynamics of the recondensing of argon vapour by pressurised $\mathrm{LN}_{2}$ is best described by the phase diagram in Figure 7.6. The normal operating pressure of the argon liquid-vapour system is slightly elevated, $\Delta P_{A r}$, to encourage vaporisation, pushing gas flow through the purifier and increasing the rate of purification. Thus, the operating temperature of the LAr is increased by $\Delta T$. The only source of cooling power in the system is from the $\mathrm{LN}_{2}$, which is pressurised such that its temperature equals that of the argon system. Given the design nominal LAr temperature of $89 \mathrm{~K}$, the normal operating pressure of the $\mathrm{LN}_{2}$ vessel is around 3.3 bar. This pressure is maintained by an accurate, adjustable back pressure regulator, thereby setting the desired nitrogen temperature. By adjusting the back pressure regulator to hold different pressures, effectively the temperature and pressure of the argon system can 


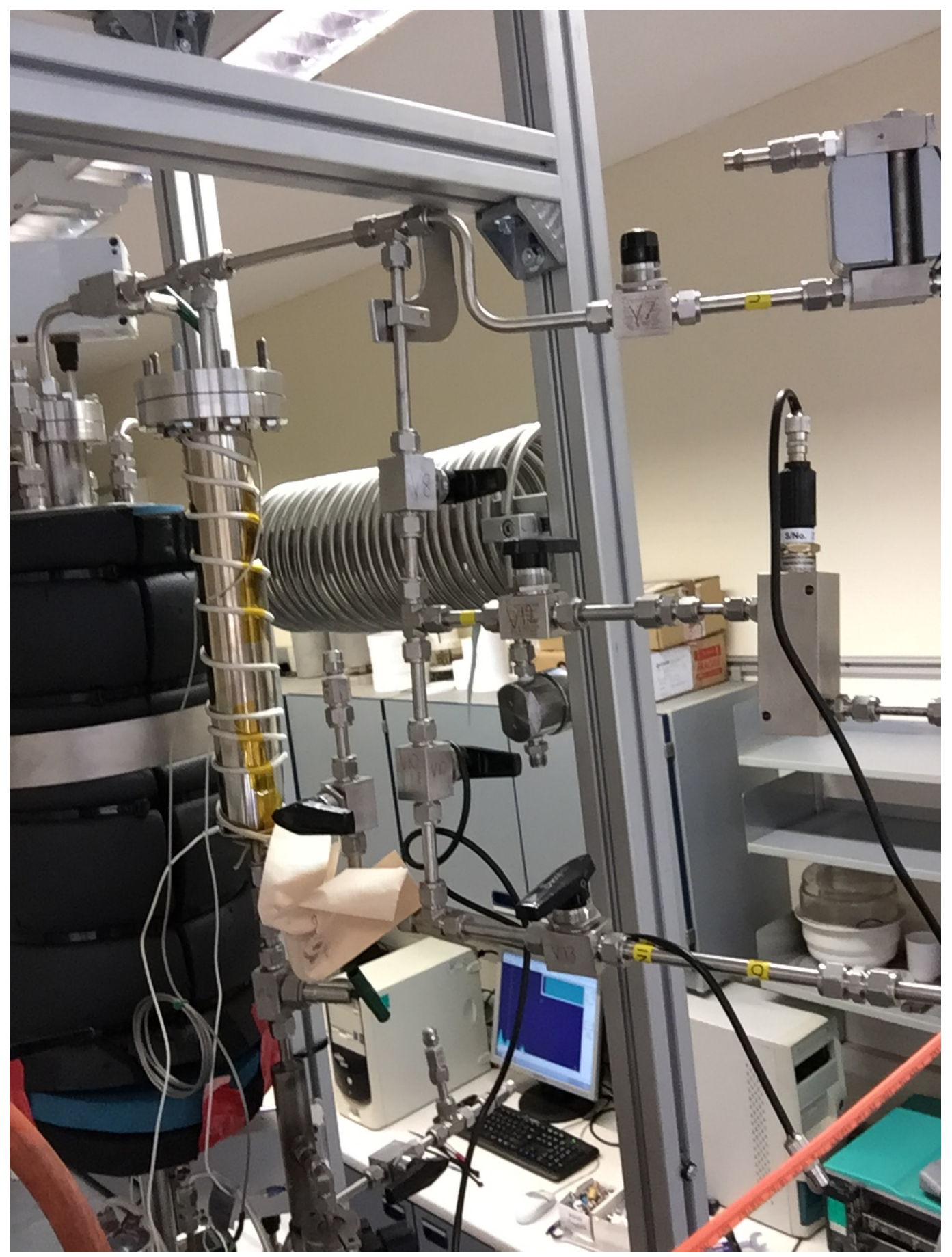

Fig. 7.4 Purifier vessel is shown as the vertically oriented cylinder with coiled heating cable wrapped around it. Also in the image, dew point sensor for monitoring the activation process (rectangular block of steel with instrument on top, right side of the image centred vertically). 


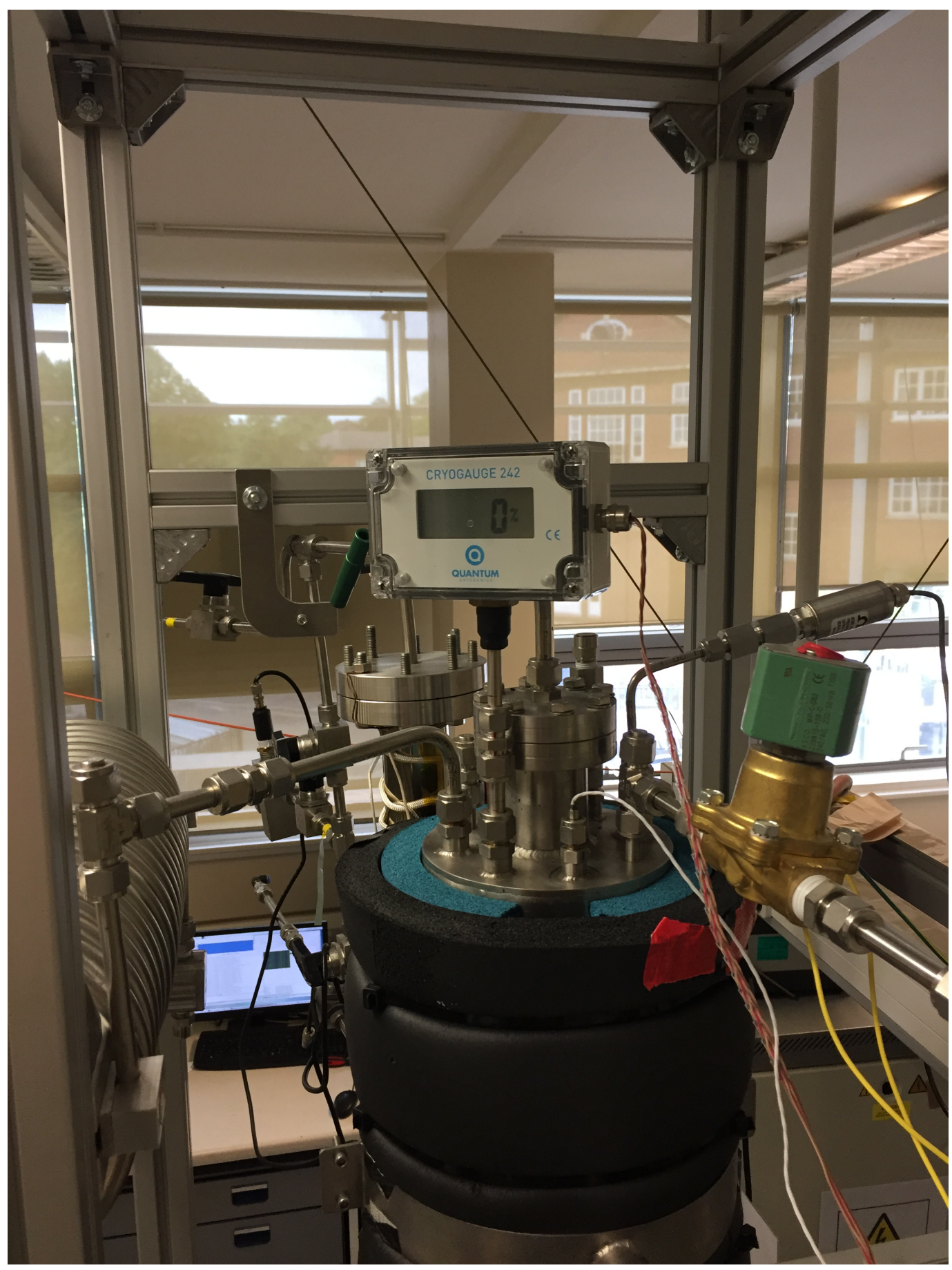

Fig. 7.5 Top of recondenser vessel (covered in blue and black insulation). Also shown: capacitive level meter and display (CryoGauge 242), solenoid valve (brass valve with grey switching element), PT100 temperature sensor (small element mounted on the near side of the vessel with white wire), and presser transducer (instrument on end of pipe which is bent at 2 o'clock, right side of image). Spiralled pipe on the left ensures the cold boiloff gas warms sufficiently before reaching the back pressure regulator, which can freeze. 
be adjusted within a reasonable range defined by the operational safety parameters of the cryogenic system.

A system of relief valves is implemented to ensure the safety of the system in case of catastrophic failure of the vacuum jacketing or insulation. The valves open if the vessel pressure exceeds a predetermined value, then reseal once the pressure drops below the predefined setpoint. The maximum allowable overpressure in the LAr vessel is $8 \mathrm{psig}$ ("pounds per square inch gauge": pressure relative to atmosphere), and the maximum pressure in the $\mathrm{LN}_{2}$ vessel is 50 psig (Valve Check part no. 220-5C-2M). The valves have a Teflon O-ring to allow resealing for reuse.

\subsubsection{Instrumentation}

The LAr and $\mathrm{LN}_{2}$ vessels are both instrumented with PT100 resistance temperature device (RTD) probes (Omega Engineering part nos. PR-10-2-100-1/4-24-E and PR-10-2-100-1/4-12E) for accurate monitoring of temperature over the full range of $-200^{\circ} \mathrm{C}$ to $30^{\circ} \mathrm{C}$. The probes are read out using the four-wire configuration to prevent the influence of line resistance on the temperature measurement. Each temperature is read out by a dedicated signal conditioner (Omega Engineering part no. CNi3253) and retransmitted as a proportional 0-10 V DC signal for reading out by the slow control.

The LAr and $\mathrm{LN}_{2}$ vessels are also both instrumented by pressure transducers (Omega Engineering part no. PX209-030A5V and PX209-060A5V) for monitoring the overpressure of the argon vapour from 0 to 30 psia ("pounds per square inch absolute": pressure relative to vacuum), and the nitrogen vapour from 0 to 60 psia. The transducers transmit the pressure as a DC voltage in the range $0-5 \mathrm{~V}$.

Capacitive level meters (CryoGauge 242) are used to measure the LAr and $\mathrm{LN}_{2}$ levels in the respective vessels, which simply measure the capacitance $C$ between two concentric cylinders. Here, the capacitance is defined as

$$
C=A\left(\varepsilon_{l} l_{l}+\varepsilon_{v} l_{v}\right)
$$

for the permittivity of the liquid, $\varepsilon_{l}$, the permittivity of vapour, $\varepsilon_{v} \neq \varepsilon_{l}$, the length of cylinder filled with liquid, $l_{l}$, the length of cylinder filled with vapour, $l_{v}$, and a proportionality constant, $A$, which depends on the geometry of the system. The fractional liquid level, $L L(\%)$, in the full cylinder of length $L=l_{l}+l_{v}$ can then be determined by simply measuring the capacitance and correcting for the differences in permittivities:

$$
L L(\%)=\frac{(C / A L)-\varepsilon_{v}}{\varepsilon_{l}-\varepsilon_{v}} .
$$




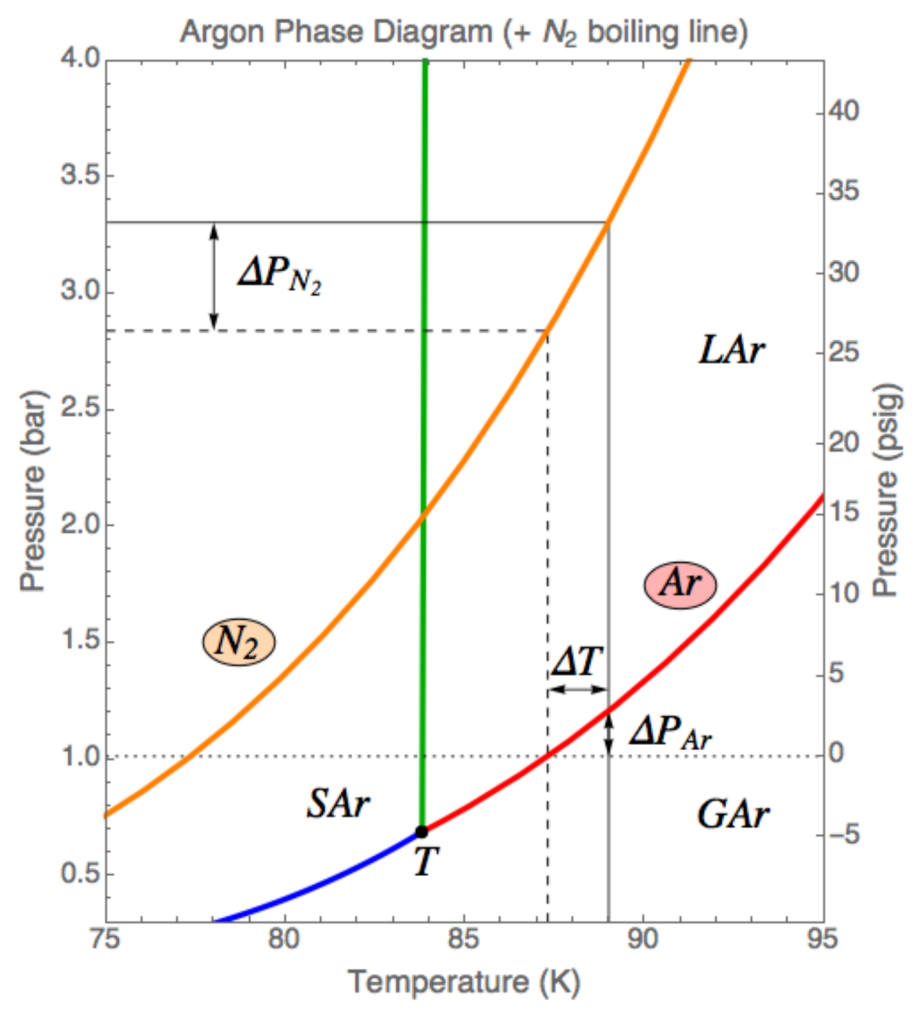

Fig. 7.6 Phase diagram of argon with nitrogen boiling line superposed [186].

The liquid level, as a fraction of the full vertical range, is calculated automatically by the two CryoGauge meters. The measurements are accessible to the slow control by the retransmission of the level as a proportional DC current between 4 and $20 \mathrm{~mA}$.

In addition to the capacitive level sensor, the LAr liquid level is also determined by measuring the differential pressure between the bottom and the top of the Dewar. The differential pressure transducer measures the hydrostatic pressure under $l_{l}$ depth of LAr with reference to the vapour pressure at the top. The transducer (GP:50 part no. 216-B-XD-AA) measures up to 30 inches of water equivalent, or about 0.075 bar, to within $0.5 \%$ of the full scale, and retransmits the pressure as $0-10 \mathrm{~V}$. Through experience, it is observed that the differential pressure transducer is more accurate at measuring the liquid level than the capacitive level meter.

In the unlikely event where the thermal insulation on the argon system is so efficient that the rate of boiloff reduces significantly, the rate of purification will drop, allowing outgassing to increase the impurity concentration of the LAr quickly. This increase can be mitigated by injecting additional heat energy into the argon system to encourage faster vapour flow rate through the purifier. Thus, a simple resistive heating element in a pipe is incorporated in 
the vessel. A maximum of $150 \mathrm{~W}$ is deliverable by using a series of $15 \Omega$ thick film power resistors embedded in an SS pipe submerged in the LAr.

In response to the loss of nitrogen mass by the venting of excess pressure through the back pressure regulator, the $\mathrm{LN}_{2}$ of the system must be periodically topped up from an external Dewar. This process is automated by the incorporation of a solenoid-operated cryogenic valve (ASCO 8222G002LT). Using LabView, a simple feedback loop was programmed (Figure 7.7a) whereby the output voltage of the $\mathrm{LN}_{2}$ level meter (liquid level) determined when the nitrogen needed to be refilled. It sent a signal to a solid state relay (SSR) to open the solenoid valve and close once filled. This way, the operation of the system may, theoretically, continue unsupervised indefinitely, as long as an uninterruptible supply of $\mathrm{LN}_{2}$ can be used.

A Servomex DF-550E gas analyser is used to measure the oxygen contamination in the argon, which has a lower detection limit of 200 parts per trillion $\mathrm{O}_{2}$ in Ar. The oxygen concentration is retransmitted as a proportional voltage for reading by the slow control.

\subsubsection{Argon Purifier}

Because the attenuation of drifting free electrons in LAr is highly sensitive to the concentration of electronegative impurities, it is especially important to maintain a high level of purity. Some particularly critical impurity species are oxygen and water. These can be removed from argon by physical and chemical reactions. Water molecules, for example, can be adsorbed by a microporous material known as a molecular sieve. By choosing a material with pores of sufficient diameter, water molecules are small enough to be adsorbed while argon atoms are not. Once saturated, the molecular sieve can be raised to a sufficiently elevated temperature that the adsorbed water vaporises and is released from its state of attachment. For oxygen molecules, however, a more efficient method of attachment is the chemical process of oxidisation. In particular, pure elements such as copper, zinc, and aluminium can undergo oxidisation, thereby reducing the concentration of oxygen in the original gas. This process can be reversed by flowing hydrogen gas across the oxidised material, releasing water and returning the metals to pure form. Therefore, such a filter can efficiently remove oxygen and water impurities from argon, and be regenerated without replacing the filter materials. The purifier described here is the standard method for many current and future LAr TPC experiments [186, 188, 189], including the Deep Underground Neutrino Experiment (DUNE).

Upon delivery, the purifier materials are in oxidised form and need to be activated before purification. The activation is accomplished by passing hydrogen through the filter, as above. The filter should be kept between $175^{\circ} \mathrm{C}$ and $250^{\circ} \mathrm{C}$ to optimise the rate of activation. Also, because the reduction process is endothermic, the hydrogen should be diluted with an inert gas, such as argon, to slow the reaction. To avoid risks related to the flammability of hydrogen 
gas, the total concentration of hydrogen in argon should not exceed $2 \%$. The reduction process is monitored by measuring the quantity of water in the output gas flow. When the outlet gas contains no more water, the filter is active, and should not be suddenly exposed to oxygen as an extremely exothermic oxidisation reaction will happen.

On the Sheffield test stand, activation of the gas requires some pieces of equipment. First, a heating tape (Omega Engineering part no. FGR-060/240V) is wrapped around the purifier cylinder to provide $250 \mathrm{~W}$ of even heating to the entirety of the filter. The filter temperature is monitored by a thermocouple (Labfacility part no. FL-K-2M) which is read out using the same temperature controller as above (Omega Engineering part no. CNi3253). Another LabView feedback loop (Figure 7.7b) is written to read the temperature of the filter, and if below (above) the activation temperature, will send a signal to an SSR to turn on (off) mains power to the heating tape.

Activation (or reactivation) is completed when the output gas stream no longer contains water. A dewpoint sensor measures this (Alpha Moisture Systems, part no. DS2000-RD-V-AHD-HD-6) which can measure water content in argon within a range of 0-1000 ppm (parts per million). The system retransmits the concentration as a proportional current within the range 4-20 $\mathrm{mA}$ for reading and logging by the slow control.

\subsubsection{Slow Control}

The slow control for the entire test stand was written in LabView and is interfaced to the computer via National Instruments (NI) hardware. In particular, voltage and current measurements from all relevant components are measured using an NI SCB-68A unit, connected to the multifunctional data acquisition system NI PXI-6229, which interfaces with the computer. Digital high and low signals may also be transmitted by the NI system to control SSRs which power the solenoid valve and heating resistors. Therefore, almost the entire operation of the test stand is automatic, apart from manually ensuring a constant supply of external $\mathrm{LN}_{2}$ by changing Dewars.

Several feedback loops are implemented in the software for controlling different subsystems. First, the purifier materials are activated by monitoring the temperature of the filter bed and by sending a digital signal to a SSR which powers the heating element. If the temperature is less than $175^{\circ} \mathrm{C}$ the heating element is powered on, and once the temperature rises above $225^{\circ} \mathrm{C}$, the heating element is turned off. A similar feedback loop automates the filling of $\mathrm{LN}_{2}$ : the solenoid valve is opened when the level falls below $30 \%$ of the maximum nitrogen capacity in the recondenser and closed when it fills to $90 \%$. These loops are illustrated in Figure 7.7. Manual control of these systems is also an option, in case more control is desired. 


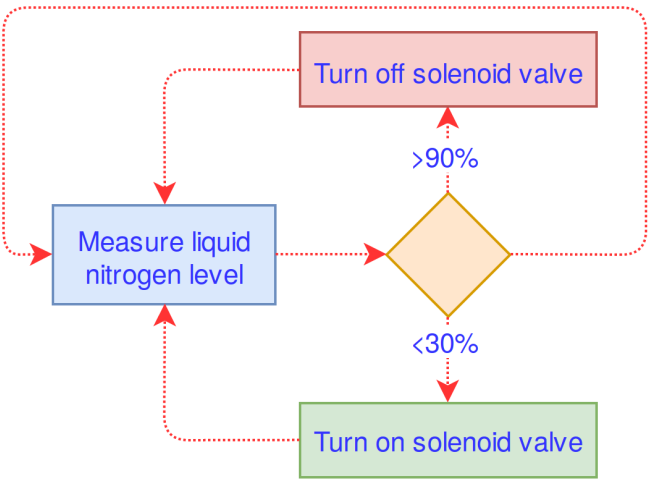

(a) $\mathrm{LN}_{2}$ control loop.

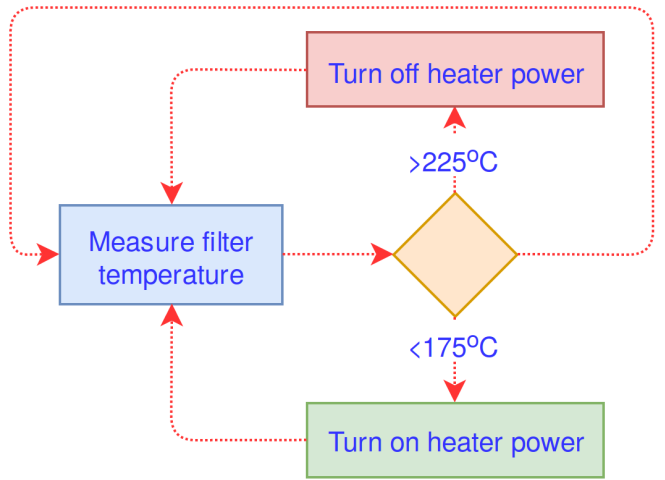

(b) Filter activation control loop.

Fig. 7.7 Slow control logic feedback loops for test stand subsystems.

The slow control LabView program also includes facilities for realtime monitoring and logging of all temperatures, pressures, liquid levels, and timestamps of control signals.

\subsubsection{Electronics Feedthrough and Cables}

Due to the general purpose nature of the test stand, a flexible electronics feedthrough system was implemented. A Conflat-40 flange was installed on the main argon Dewar lid, to which any required system of high voltage (HV) or instrumentation feedthroughs could be connected. For initial experiments in the test stand, a four-way Conflat- 40 cross was attached, the three available ports contained a 12-pin HV feedthrough, a 3xSHV feedthrough, and a vacuum pressure gauge.

Materials for cabling and construction of elements inside the Dewar are chosen to not significantly outgas impurities into the LAr. These include polyetheretherketone (PEEK), polytetrafluoroethylene (PTFE), SS, and Kapton. These and other suitable materials have been tested for use in a high purity LAr volume and are shown not to increase the electron drift lifetime substantially [189].

\subsection{Operation}

The standard operating procedures for the test stand can be summarised in a few steps, which are described more fully in [187]. These steps should be followed once a fully functional electronics and slow control system was installed, as problems usually cannot be fixed without having to restart all operating procedures from the beginning. 
First, the system must successfully pass a helium leak check by a qualified person and with a calibrated detector. This requirement is to ensure that no air can backflow into the argon volume and contaminate the liquid supply.

Next, the entire system should be thoroughly insulated with Armaflex AF and LTD foam insulation. Notably, elements which are not vacuum-jacketed should be wrapped. For instance, the $\mathrm{LN}_{2}$ Dewar, the filter container, all argon piping, and the liquid feed pipes from the external Dewar.

Once insulated, the entire system should repeatedly be pumped and purged thoroughly with argon gas to remove air. It is recommended that four cycles are sufficient to reduce the air content to about 1 part in $75^{4}$.

At this point, the filter can be activated by flowing $\mathrm{H}_{2}(5 \%) / \mathrm{Ar}$ across the heated bed of materials, as described in Section 7.1.3.

Once the system is sealed, purged of air, and the filter activated, the vessel is ready for cooldown and filling of LAr. This process begins by opening the valve to fill using an external Dewar supply of LAr. This process is critical as the rate of cooldown must be sufficiently slow to prevent thermal shock of the delicate detector components inside the Dewar. By hand adjusting the flow of the supply, arbitrary speeds of cooldown can be achieved, depending on the requirements of the equipment. $1^{\circ} \mathrm{C}$ per hour is usually a sufficiently slow cooldown rate. Once the vessel has cooled to the boiling point of argon, it will begin filling with liquid.

After the LAr vessel is filled, the $\mathrm{LN}_{2}$ vessel should be filled so that purification and recirculation can occur. Once this is completed, the pressure of the $\mathrm{LN}_{2}$ system backpressure regulator should be adjusted to balance the thermodynamics of the system, as described in Section 7.1.1.

Normal operation of the system involves continuous refilling of the $\mathrm{LN}_{2}$ Dewar as it is expelled from the back pressure regulator. The rate of refilling depends directly on the level of heat loss in the system. More insulation would encourage more gradual argon and nitrogen vaporisation, and slower nitrogen loss to the atmosphere. Given the level of automation in the nitrogen fill, and the closed argon system, normal operation can theoretically continue indefinitely, as long as a constant $\mathrm{LN}_{2}$ supply can be maintained.

Warm up of the system occurs by first sealing the filter bed in an inert oxygen- and moisture-free environment. Then, the nitrogen and argon vessels can be directly vented to atmosphere, or drained for recycling.

In May 2016, a full cooldown of the test stand was completed to verify the engineering design and functionality. The full complement of process measurements for the first several hours is shown in Figure 7.8. For three hours, the stable operation of LAr recirculation and recondensing was demonstrated. The stability is shown by the continuous refilling of 
the $\mathrm{LN}_{2}$ vessel as it boils off. The LAr capacitive level measurement and the differential pressure measurement are highly correlated, demonstrating the efficient functionality of the two separate systems in measuring the same process.

The slow decrease in LAr level throughout the test run is thought to be caused by a slow leak in the LAr relief valves. While they were designed such that the valves could be reseated in the PTFE O-rings, the reseated valves are not guaranteed to be perfectly vacuum-tight and would degrade after many bursts and reseals. For future runs, the relief valves will need to be replaced to maintain the supply of purified argon for more extended periods of time.

\subsection{Summary}

The LAr Test Stand at Sheffield is a multipurpose facility for fundamental studies of the properties of LAr and particle detectors. By recirculating the argon and using continuous filtering of impurities, very high levels of purity can be achieved, allowing for the transporting of electrons long distances for detection. Also, the automation and balanced thermodynamics of the system provides long-term operation with minimal manual interference required. The test stand has been commissioned and is ready for use. 

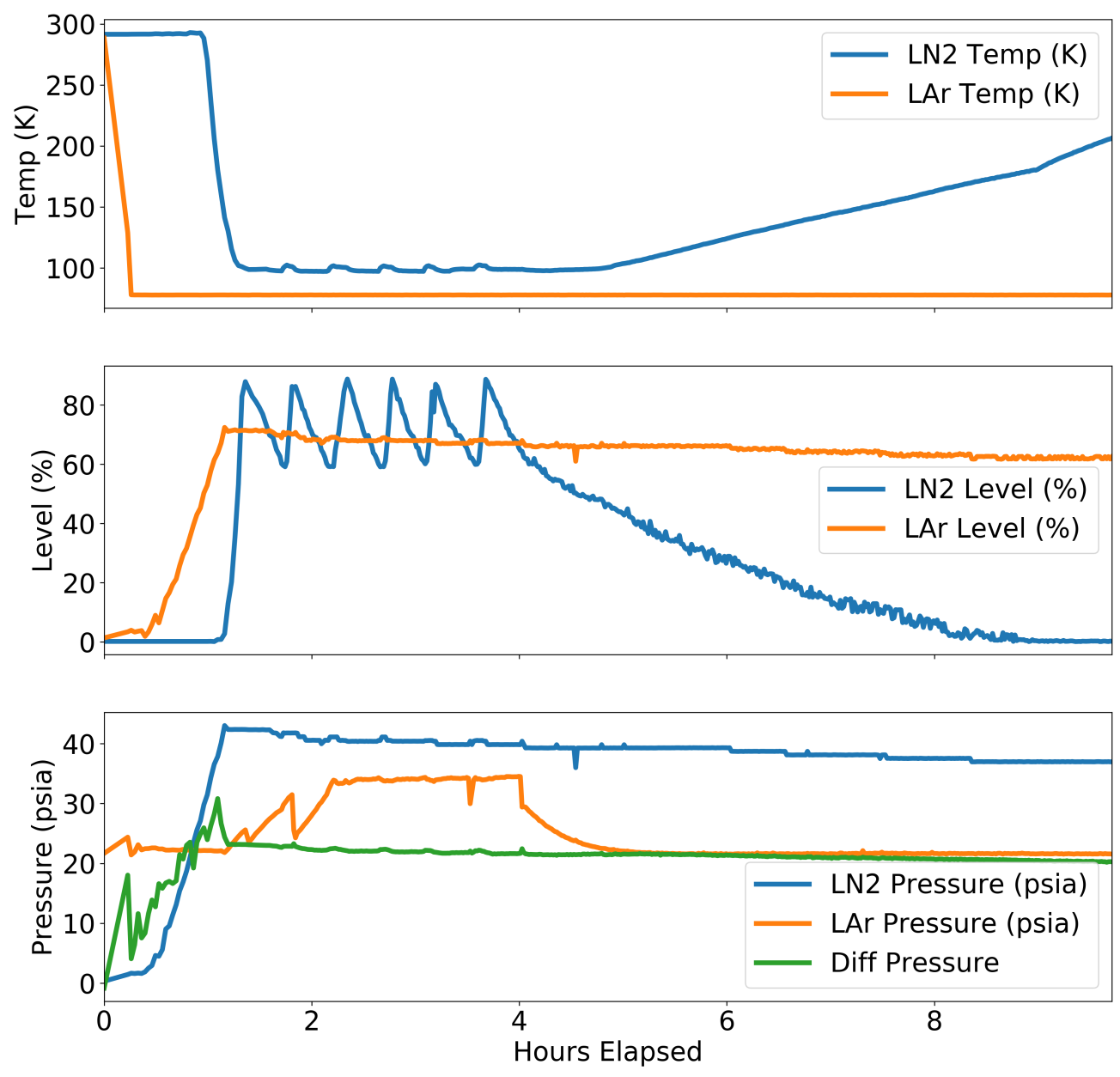

Fig. 7.8 Process measurements for the first engineering cooldown test. The stable LAr and $\mathrm{LN}_{2}$ system thermodynamics was demonstrated for three hours before warm up. Hour 0 Argon fill begins. Hour 1 - Nitrogen fill begins. Hours 1-3 - Normal operation. Hour 4 - Test ended, warm-up of the system begins. 


\section{Chapter 8}

\section{Cryogenic Gaseous Photomultiplier Development}

As noble liquid detectors become more prevalent and important in particle physics, more practical applications of the technologies are being pursued, such as using LAr or LXe as scintillating materials in positron emission tomography (PET) as an alternative to crystals [183]. The position resolution of current PET scanners is limited by the granularity of the crystals and the photomultipliers (PMT), but this can be significantly improved by using a liquid scintillator and high-resolution position-sensitive detectors. The scintillation light yield of liquid argon (LAr) (51 photons/keV [190]) and liquid xenon (LXe) (40 photons/keV [191]) is similar or better than conventional PET crystals (e.g. NaI(T1): 38 photons/keV, BGO: 6 photons/keV [192]) allowing similar or better energy resolution.

The position-sensitive gaseous photomultiplier (GPM) is a powerful new type of detector which combines the single photon sensitivity of a conventional PMT, direct sensitivity to vacuum ultraviolet (VUV) light without wavelength shifting, and the position sensitivity of a high-density pixelated imaging detector. The GPM can be manufactured with a substantial active area, so provides a cheaper alternative to PMTs and silicon photomultipliers (SiPM) while still improving the position sensitivity without loss of efficiency due to wavelength shifting VUV light to the sensitive region of the detector.

Position sensitive GPMs have been shown to be stable at cryogenic temperatures [193, 194], though most of the literature tests GPM detectors in LXe, rather than LAr. There is an excellent reason to consider LAr as a detection medium, rather than LXe, despite its lower density and stopping power. For instance, LAr is cheaper and has very similar scintillation light yield to LXe [190, 191]. Also, the scintillation photon energy for LAr is higher than for LXe, leading to better quantum efficiencies from certain photocathodes such as caesium iodide (CsI) [195]. 
The goals of the tests in this chapter are to demonstrate the GPM's structural integrity, gas electron multiplication performance, and sensitivity to VUV scintillation light in LAr. Firstly, the design and construction of the detector are described, as well as the data acquisition and analysis systems. The GPM is characterised by first confirming room temperature operation of the detector, then testing it at an intermediate cryogenic temperature using solid $\mathrm{CO}_{2}$, testing the VUV sensitivity of the photocathode, and finally by testing it at a temperature near that of LAr while submerged in liquid nitrogen $\left(\mathrm{LN}_{2}\right)$.

\subsection{Micro-Patterned Gaseous Detectors}

As with noble liquids (see Chapter 2), energetic particles may excite and ionise noble gas atoms, creating electron-ion pairs and emitting deexcitation photons (i.e. scintillation). In the presence of a strong electric field, the ionised electrons can accelerate and, if the field is strong enough, may cause secondary ionisation and excitation through collisions with atoms in the medium. The secondary ionisation and scintillation processes cause an avalanche, exponentially increasing the charge and light available for detection. Thus, single primary electrons (or photons) can be detected through the multiplication of secondary signal, as in a PMT. Additionally, the energy deposited by the primary particle is proportional to the number of scintillation photons or ionisation electrons emitted in the interaction with the medium. If it is stopped in the medium, the primary particle energy is therefore proportional to the collected charge or secondary scintillation light after multiplication in the gas [35].

The Townsend equation describes the gas multiplication process:

$$
\mathrm{d} N=\alpha N_{0} \mathrm{~d} x,
$$

which defines the number increase in electrons, $\mathrm{d} N$, over some distance, $\mathrm{d} x$. The Townsend coefficient, $\alpha$, depends on the properties of the medium and the electric field applied. Above the threshold for excitation and ionisation, $\alpha$ increases monotonically with the applied electric field. For a strong uniform electric field, the solution to Equation 8.1 becomes

$$
n(x)=n_{0} e^{\alpha x} .
$$

By multiplying the number of electrons by the elementary charge, $q_{e}$, the total resulting charge after multiplication over a distance, $d$, becomes $Q=n(d) q_{e}=q_{e} n_{0} e^{\alpha d}$. For an arbitrary number of primary electrons to start the avalanche, $n_{0}$, the gain $G$ is then defined as

$$
G=Q /\left(n_{0} q_{e}\right)=e^{\alpha d}
$$


It is shown that some factors such as ion backflow can affect the total gain, as the slow-moving positive ions can shield the electric field applied in the avalanche region, or recombine with the electrons reducing the total collected charge [196]. Additionally, this multiplication process has an upper limit where the exponential increase in charge becomes more similar to an electric discharge, which is known as the Raether limit [197, 198].

The charge multiplication process requires that the mobility of electrons in the gas is not low. Noble gases are ideal as they are inert and do not themselves attach to free electrons. However, one remaining problem with the gas multiplication process in a noble gas is the production of secondary scintillation light. Such photons, in general, escape the main path of the avalanche and interact in other parts of the detector creating spurious pulses and leading to the loss of energy linearity. In short, secondary scintillation reduces the proportionality of the multiplication process, leading to loss of energy and position resolution. This problem can be mitigated by the addition of a small amount of a polyatomic electronegative gas, such as methane $\left(\mathrm{CH}_{4}\right)$. These so-called "quenching" gases are excited by the scintillation photons, but the energy is dissipated as heat, rather than causing further spurious ionisation [20].

One type of detector which is well-suited to take advantage of the gas multiplication process is the thick gas electron multiplier (THGEM) [184], a variant of the GEM [199]. It consists of a roughly millimetre-thick insulator such as a standard printed circuit board (PCB), with electrodes printed on both sides and holes drilled through to allow the passage of free drift electrons (see Figure 8.1). A voltage is applied across the electrodes, which creates a high electric field in the holes through which electrons may avalanche. Stacking multiple THGEMs on top of one another, forming numerous stages of multiplication, is one way to increase the gain of the system further while reducing the risk of electric discharge.

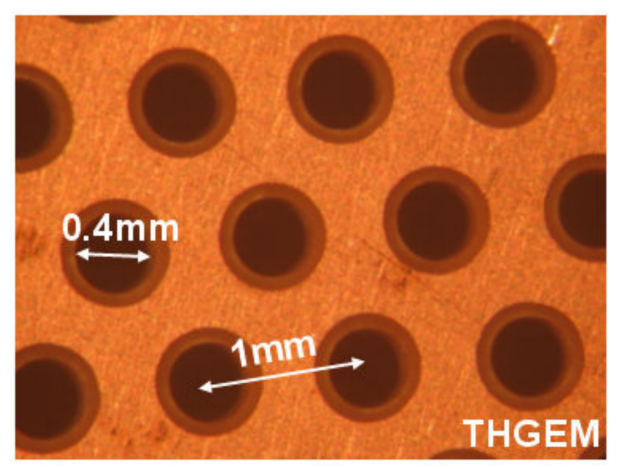

Fig. 8.1 Photo of typical THGEM electrode. 
Position-sensing of the multiplied electron cloud can be accomplished using a strip-based readout. The thick COBRA ${ }^{1}$ (THCOBRA) [200] (see Figure 8.2) was designed to perform the same charge multiplication functions as a THGEM, but to also provide 2D position readout by segmenting the electrodes on top and bottom of the PCB into strips. On one side (the "top") of the THCOBRA, many parallel strips are extended to one end of the PCB and are connected to a resistive line. As an electron cloud passes through a hole of the THCOBRA, the moving charge induces a current pulse in the strip, whose position can be determined on the resistive line using the principle of resistive charge division [201]. When the electron cloud emerges from the other side of the PCB, it follows the field lines in a U-turn across cathode strips, to the anode strips, which are alternating on the bottom of the THCOBRA. The currents induced in the anode strips as the charge is collected can be read out in the same way as the top strips, using a resistive line. Because the top strips and the anode strips are oriented perpendicular to each other, accurate $2 \mathrm{D}$ determination of the electron cloud location is possible.

\subsubsection{Photocathodes}

In some applications of gaseous particle detectors, it is desired to directly detect energetic $(O(10 \mathrm{eV}))$ photons. For example, LAr primary scintillation light $(\lambda=127 \mathrm{~nm}, E=9.76 \mathrm{eV})$ is particularly interesting for current large-scale LAr TPC experiments (see Chapter 2). By placing a solid photosensitive scintillator in the gaseous volume, a THGEM-based micropatterned detector can behave as a photomultiplier. Such scintillating materials are chosen to have a low work function allowing electrons to be efficiently extracted via the photoelectric effect, which can then be multiplied. CsI is one such photocathode, which has a sufficiently low work function, and high quantum efficiency for VUV photons (see Figure 8.3). It can be evaporated onto the surface of a THGEM, allowing primary LAr scintillation photons to be easily converted to charge for multiplication. Furthermore, this photocathode does not require any wavelength-shifting for LAr scintillation, which would be necessary with a traditional glass PMT.

CsI photocathodes are sensitive to significant degradation over time as a result of exposure to water [202]. The hydrolysis reaction causes the caesium and iodine atoms to dissociate, which degrades the quantum efficiency of the diatomic scintillator. Studies have shown a 50\% reduction in quantum efficiency after exposure to air for 100 minutes at a relative humidity of $50 \%$ [203, 204]. Other factors such as extreme photon flux and ion bombardment are also known to degrade the performance of CsI photocathodes [202].

${ }^{1}$ COBRA is not an acronym, but rather just a reference to the "snake-like" zigzagging top strips, as in Figure 8.2 (right). 


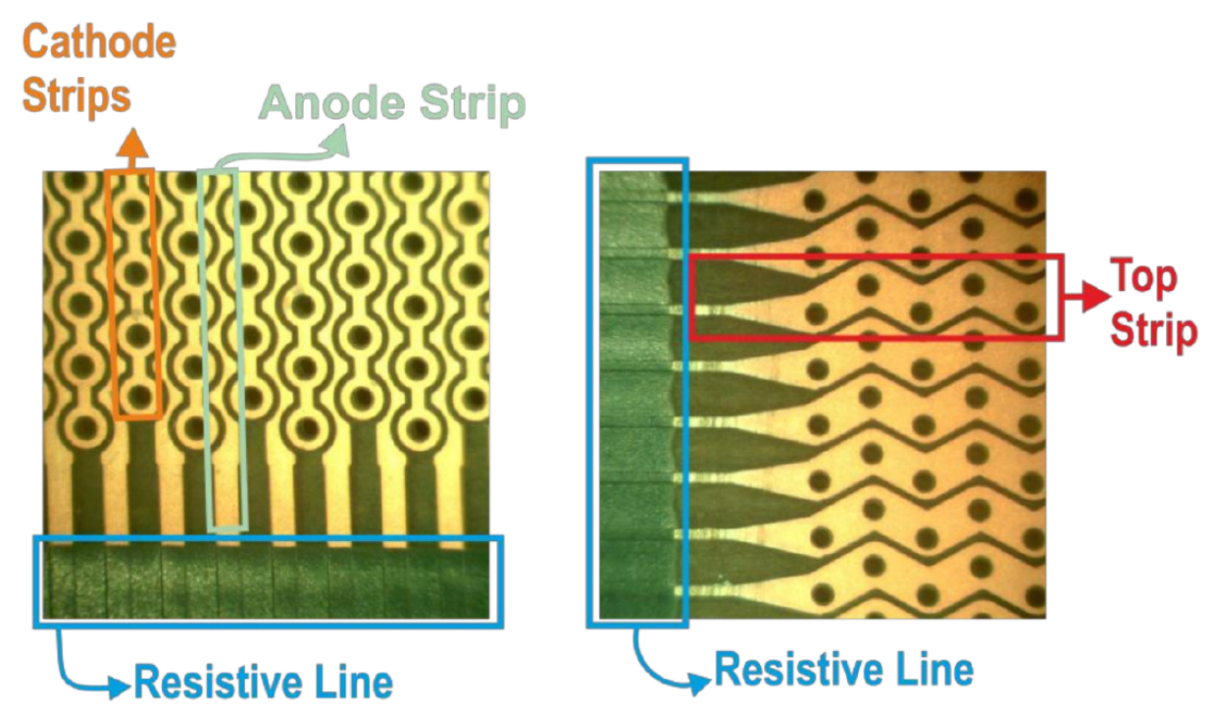

Fig. 8.2 Closeup photos of THCOBRA top strips (right) and anode/cathode strips (left).

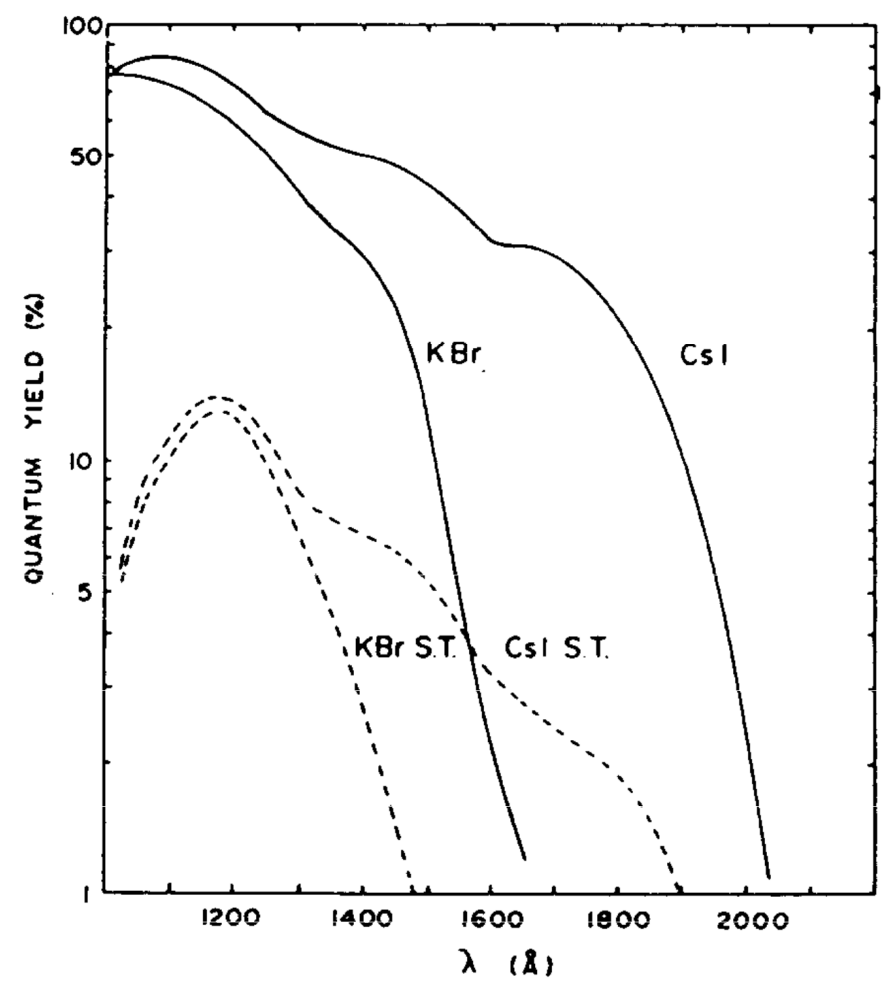

Fig. 8.3 Quantum efficiency of semi-transparent (S.T.) and reflective photocathodes of CsI and $\mathrm{KBr}$ versus light wavelength [202]. 


\subsection{Detector Description}

The GPM used in the work described here is the same detector used in [185]. The detector is housed in an aluminium cylinder (10 cm diameter and $10 \mathrm{~cm}$ length) with a $1.5 \mathrm{~mm}$ thick magnesium fluoride $\left(\mathrm{MgF}_{2}\right)$ window on one end, and gas and electronic feedthroughs on the other (see Figures 8.4 and 8.5). Diametrically opposed gas pipes are installed to fill the detector volume with the chosen gas mixture or to allow a continuous flow. All components are sealed with Teflon, which provides vacuum-tightness at room temperature.

The $1.5 \mathrm{~mm}$ thick Crystran $\mathrm{MgF}_{2}$ window has a high transmittance for VUV photons in the same region as LAr scintillation light: $T>50 \%$ at $121 \mathrm{~nm}$. The three stacked micro-patterned structures (two THGEMs, and a THCOBRA) are mounted with $3 \mathrm{~mm}$ separation between stages. The holes are all $0.4 \mathrm{~mm}$ in diameter, with $0.8 \mathrm{~mm}$ pitch on the THGEMs, and $1 \mathrm{~mm}$ pitch on the THCOBRA. The conductive strips of the THCOBRA are $0.9 \mathrm{~mm}$ wide and joined by resistive lines. A wire mesh is installed $2 \mathrm{~mm}$ above the first THGEM to allow light to pass and to shape the electric fields in the region just above the first electrode. On the top electrode of the first THGEM, a layer of CsI is evaporated, which has a quantum efficiency greater than $60 \%$ for LAr scintillation light [195]. A solid copper plane is installed $2 \mathrm{~mm}$ below the last THCOBRA, also to shape the electric fields in the region below the THCOBRA. The detector is typically operated in flow mode using $\mathrm{CH}_{4}(5 \%) / \mathrm{Ne}$, which serves as the multiplication medium.

\subsection{Operating Principle}

The GPM is based on a three-stage cascaded THGEM concept, with a VUV-sensitive photocathode, and strip-based position readout using a THCOBRA (see Figure 8.6). In this detector, a VUV photon which passes through the $\mathrm{MgF}_{2}$ window may interact in the CsI photocathode. If the photon is absorbed photoelectrically, an electron is released and, with some probability, may be ejected from the surface into the $\vec{E}_{\text {drift }}$ region in Figure 8.6. That electron would follow the electric field lines created by biasing the electrodes of the THGEMs and the THCOBRA, denoted by the thick arrows in Figure 8.6. As it accelerates through the first THGEM hole, collisions with neon atoms cause an avalanche. The resulting cloud

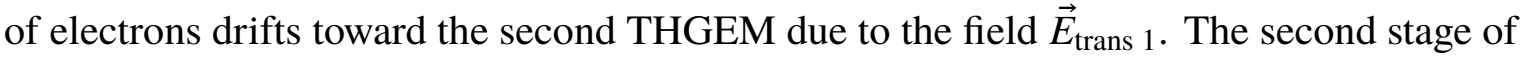
multiplication occurs in the middle THGEM and then drifts toward the THCOBRA in the

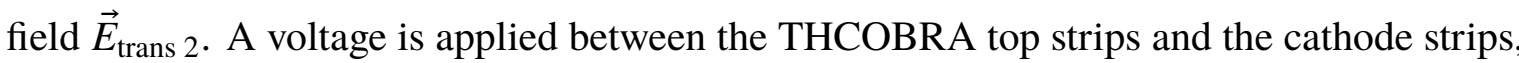
$V_{C T}$, which creates a field in which the third primary stage of electron multiplication happens, $\vec{E}_{\text {THCOBRA. The final stage of amplification occurs as the electron cloud changes direction and }}$ 


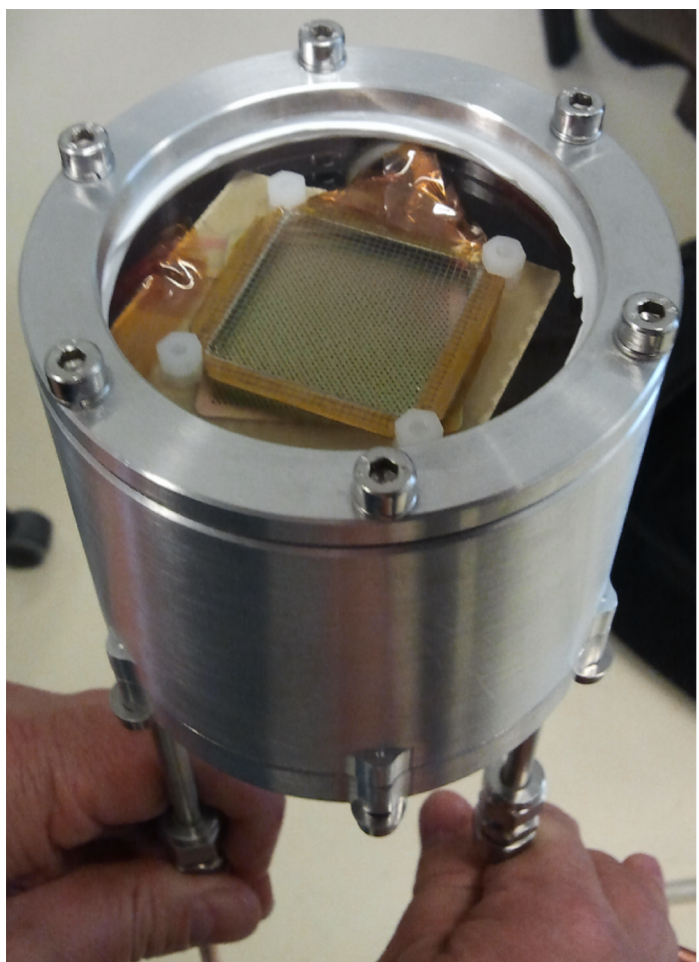

Fig. 8.4 Photo of GPM front face. Through the $\mathrm{MgF}_{2}$ window can be seen the extraction grid and the top of the first THGEM which is coated in CsI (green).

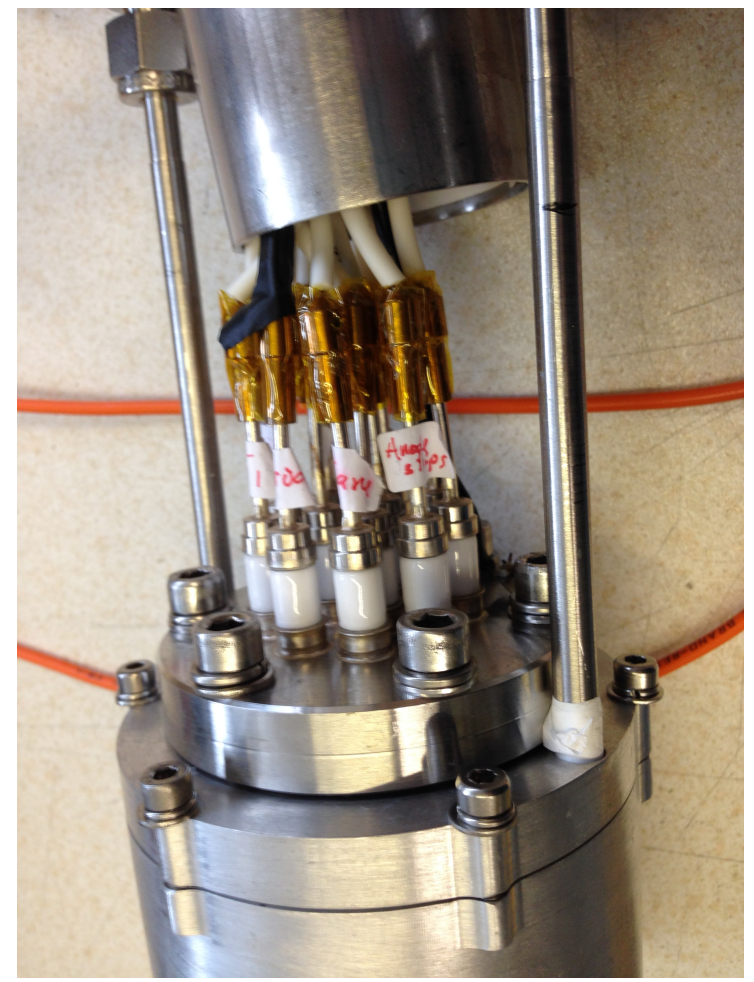

Fig. 8.5 Photo of GPM rear face, showing 12pin HV CF-40 flange with signal connections, and two gas flow pipes. 


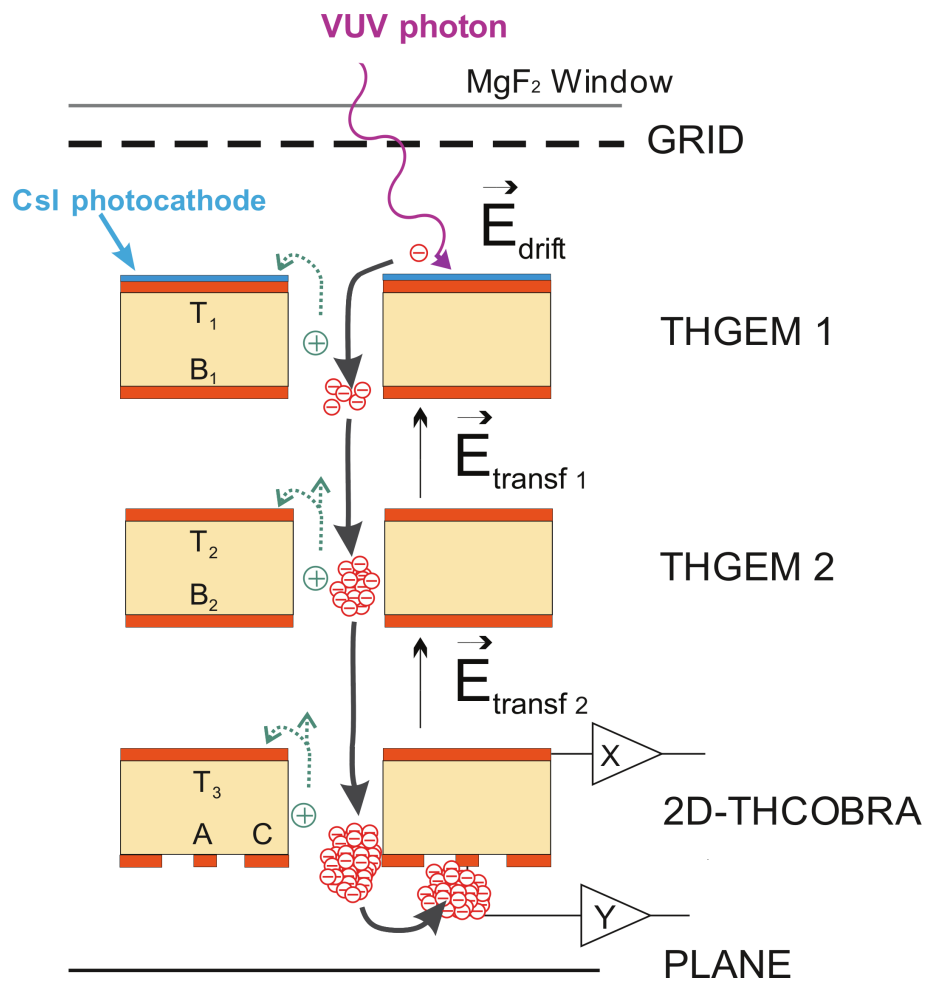

Fig. 8.6 Diagram of GPM operation.

drifts toward the anode strips under the influence of the field created by the voltage between the cathode and anode strips, $V_{A C}$. By measuring the pulse heights from both ends of each resistive line, the 2D position of the VUV photon on the photocathode can be determined.

The measurable signals produced by the GPM are in the form of induced current pulses from the THCOBRA top and anode strips (see Figure 8.7). For the top strips, an electron cloud with a total charge of $Q$ moves toward the top strips, but a charge-multiplied cloud with a total charge $>Q$ moves away from the top strips as it passes through the holes. Therefore, a positive voltage pulse is induced in the top strips. For the anode strips, however, as the electron cloud moves toward the electrode, a negative voltage pulse is induced where the field lines originate, according to the Shockley-Ramo theorem (Equation 2.2).

\subsection{Data Acquisition}

\subsubsection{Front End Electronics}

The raw signals from the GPM are processed in several steps (see Figure 8.8). First, Cremat CR-111 charge sensitive preamplifiers (CSP) are used to amplify the current pulses for further processing. A photo of the CSPs is shown in Figure 8.9 and their output in Figure 8.7. The 


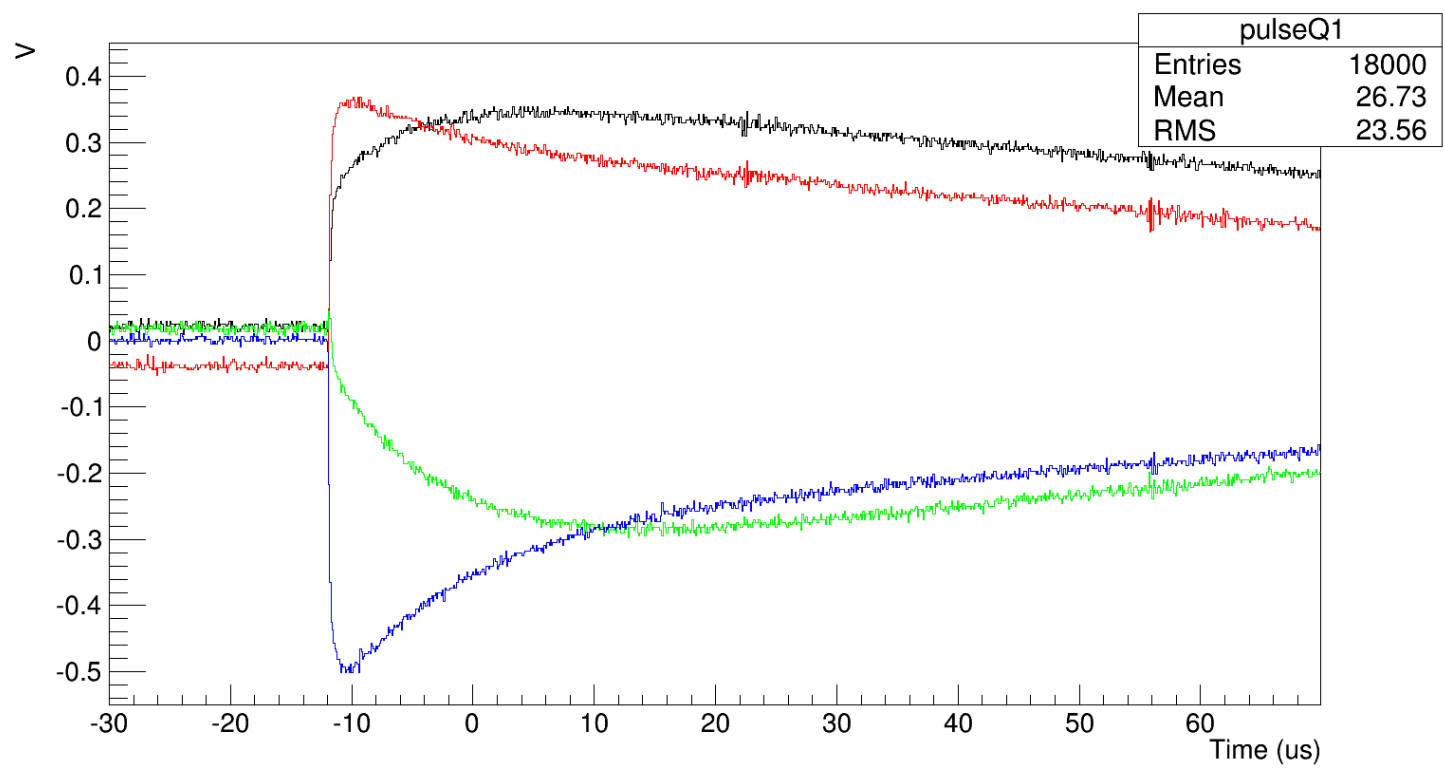

Fig. 8.7 Representative GPM pulses following preamplification but before Gaussian shaping. Two anode strip signals are shown (blue, green), and two top strip signals are shown (red, black).

preamplified pulse is further processed using Cremat CR-200-8 $\mu$ s shaping amplifiers, which have $8 \mu$ s shaping time. Figure 8.10 shows a photo of the shaping amplifiers and the readout electronics. The resulting shaped pulse is a Gaussian whose amplitude is proportional to the total amount of charge in the original raw current pulse.

Voltage is supplied to the GPM components using CAEN V6521N and V6521P HV power supplies. Both HV and signal currents use the same wires to connect the GPM with the front end electronics. The wire-sharing is achieved by capacitively coupling the HV to the preamplifier using $10 \mathrm{nF}$ capacitors.

The shaped waveform is recorded by a Keysight U5309A PCIe digitiser, which has eight channels, a sampling rate of $1 \mathrm{GS} / \mathrm{s}$, and 8-bit resolution. The unit has a builtin programmable edge trigger, which is used to find pulses in a continuous stream of data. The digitiser is installed in a Linux desktop computer, which controls the data acquisition, storage, and processing.

Following the stages of electron multiplication in the THGEMs and THCOBRA, the resulting cloud of electrons induces a current on the top and anode strips. The shaping amplifier pulse height is proportional to the number of electrons in the cloud. The proportionality constant is determined through calibration of the electronics using a fast rise time square wave pulser and an accurate $10 \mathrm{nF}$ capacitor, which simulates the injection of charge to the 


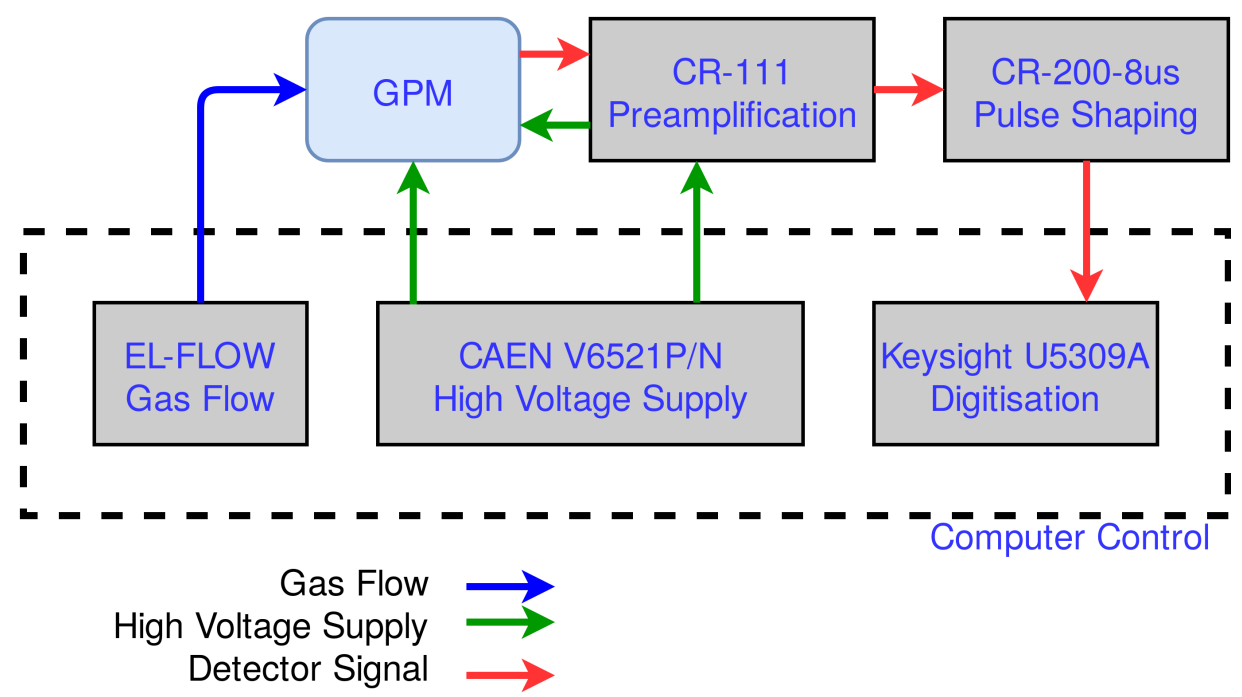

Fig. 8.8 Block diagram of front end electronics and interfaces between system components.

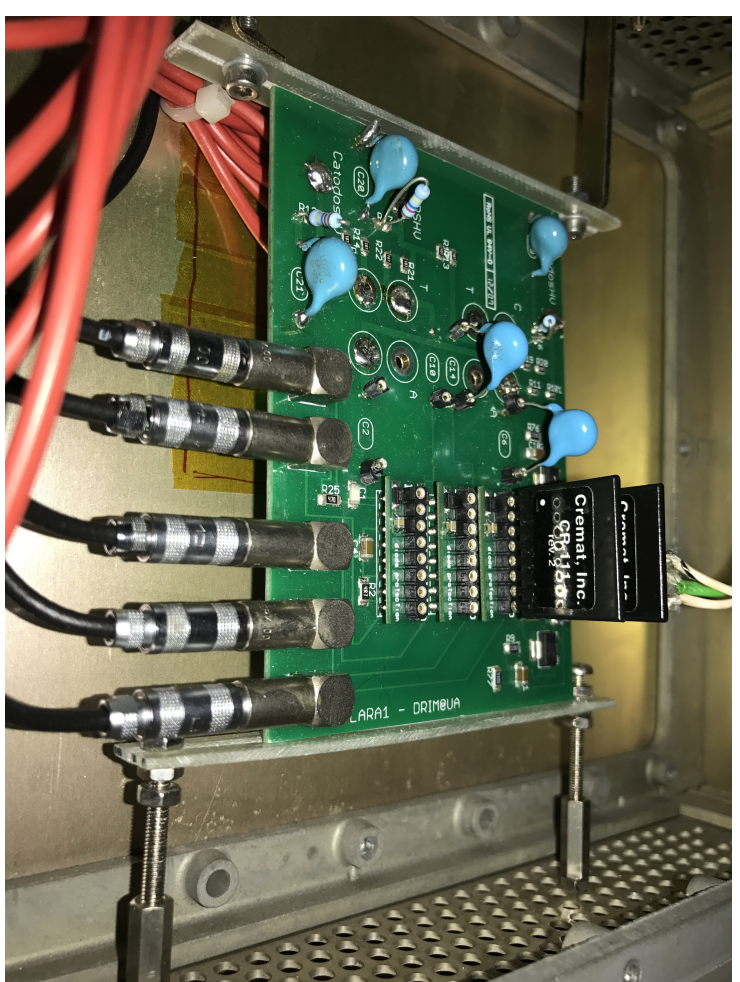

Fig. 8.9 Four channel CSP readout board showing two channels in use. Base circuit board was designed and manufactured at the Universidade de Aveiro. Circuit is installed in NIM module for electromagnetic shielding and stable DC power supply.

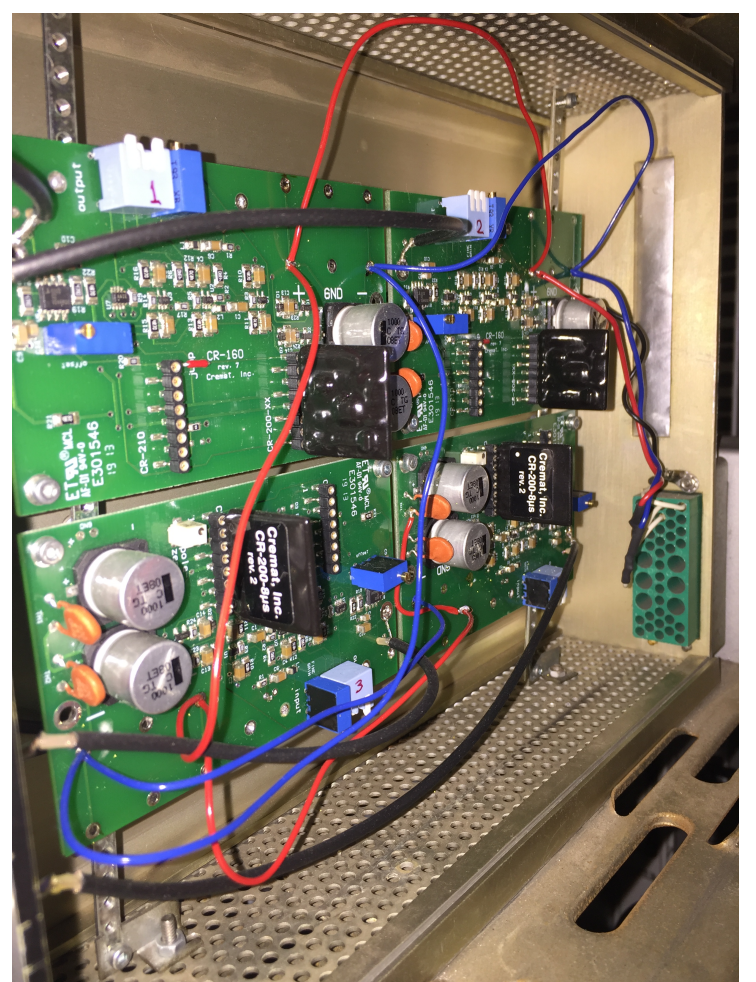

Fig. 8.10 Four Cremat CR-160 evaluation boards with which to read out CR-200 shaping amplifiers, which have optional coarse and fine gain manual controls. Boards are installed in double-wide NIM module for electromagnetic shielding and stable DC power supply. 
preamplifiers. The calibration constant of the electronics for this study is calculated as in Figure 8.11 for the anode and top strips separately.

The GPM multiplication gas flow is regulated by a mass flow controller (Bronkhorst EL-FLOW Base model no. F-201CB-500-AAD-00-V) which can provide up to $400 \mathrm{~mL} / \mathrm{min}$ of gas flow. A diagram of the layout of the gas flow system is shown in Figure 8.12. The outlet pipe is submerged in a flask of oil to visually monitor the flow of gas through the GPM.

\subsubsection{System Control and Analysis}

Custom built software which communicates directly with the various hardware application programming interfaces (API) is used to control the GPM HV, data acquisition, and analysis. These software packages, developed by the author of this work, are high-level experiment control routines intended to automate and streamline the experimental process. It is important also to note that these are entirely generalisable for other experiments which may use the same digitiser and HV hardware, not just the GPM experiment.

The control of the Keysight digitiser uses the Agilent MD2 driver for Linux, version 1.12. The driver provides a set of wrapper functions to perform actions such as initialise communication with the hardware, set acquisition parameters such as the number of samples per record and number of channels to acquire, initiate triggering, and acquiring digitised waveforms. The application developed for this experiment [205] is written in C++ and uses the provided library functions from Keysight to start and complete an acquisition based on user parameters defined in a plain text file.

Recorded waveforms are acquired from the hardware as $\mathrm{C}$ arrays with 8-bit (1-byte) data type. Each waveform uses 0.1 megabytes of disk space on the computer and is saved to a binary-formatted file for most efficient storage. For each waveform that is collected, an 81-byte binary header is also saved, which contains essential information about the acquisition parameters including, for example, TDC conversion to time parameters, ADC conversion to voltage parameters, and digitiser channel number. The general format of the contiguously saved output file is illustrated in Figure 8.14.

Another developed application reads the binary data files and performs signal processing [206]. By knowing the format of the saved header and data acquisitions, the binary files can be unzipped and the data retrieved. In particular, the header information is used to extract each waveform into a $\mathrm{C}++$ vector and to convert the $\mathrm{ADC}$ values to voltages.

Each triggered waveform is expected to contain a pulse of some amplitude which depends on the gain of the detector, the energy of the primary ionisation, and the $2 \mathrm{D}$ position of the avalanche on the THGEM stack in the GPM. Along with reading the binary data files, the 


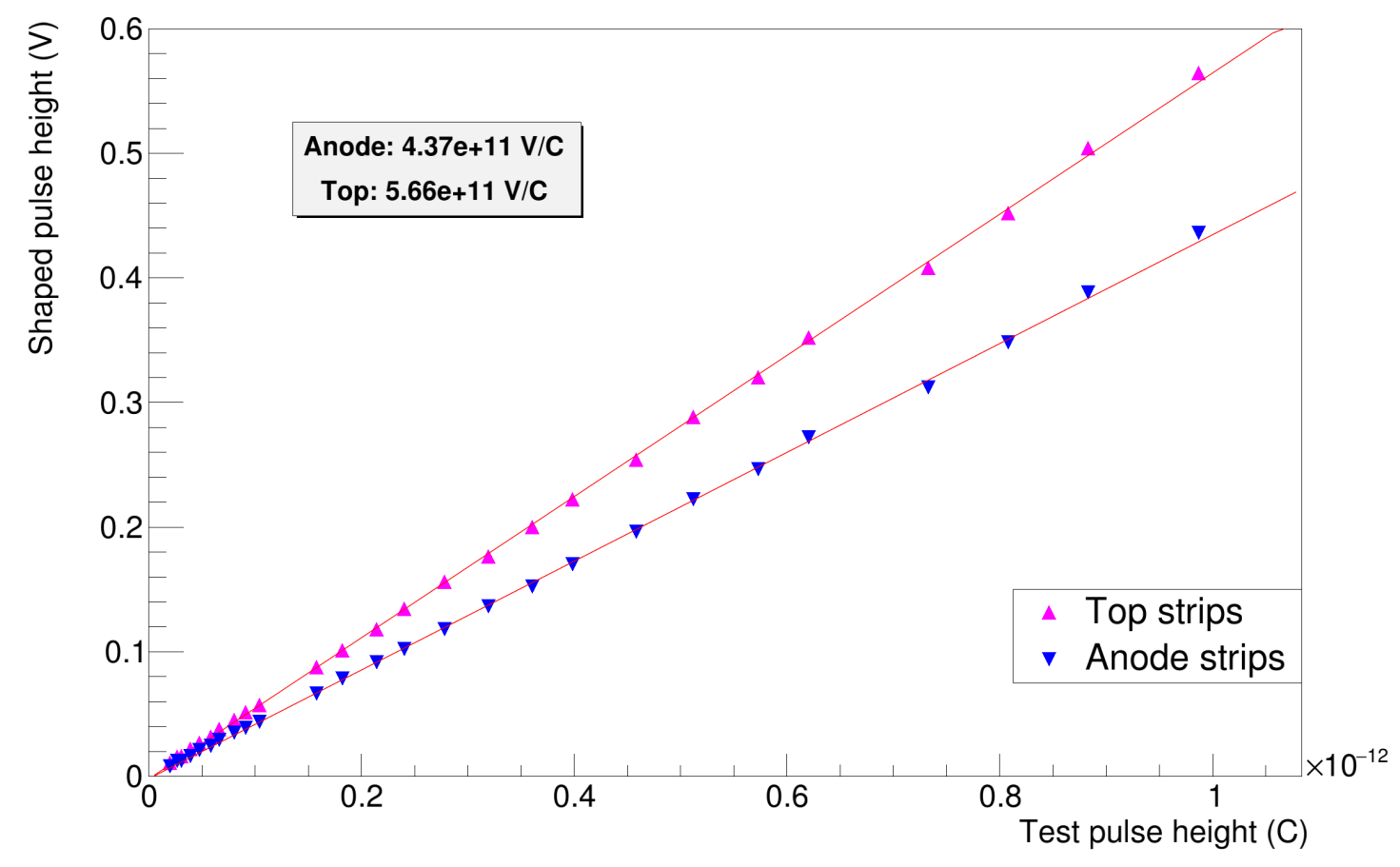

Fig. 8.11 Calibration of Cremat CR-111 premplifiers, AC coupled with $10 \mathrm{nF}$ capacitor, and Cremat CR-200-8 $\mu$ s shaping amplifier. Horizontal axis is calculated as $10 \mathrm{nF}$ multiplied by the input step voltage. 


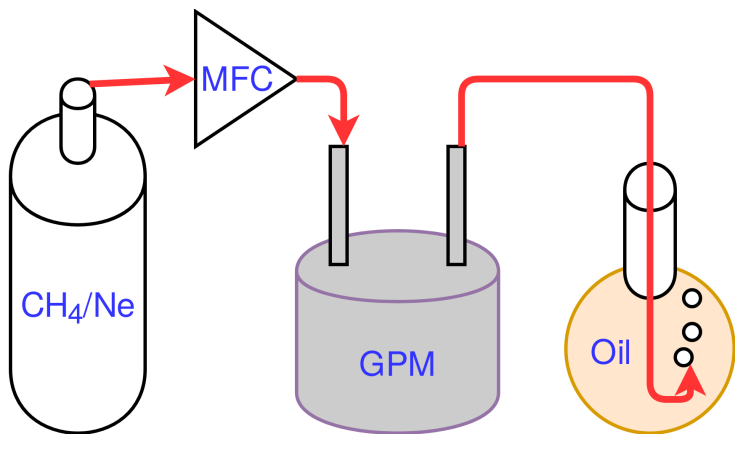

Fig. 8.12 Left to right: 155 bar supply cylinder of $\mathrm{CH}_{4}(5 \%) / \mathrm{Ne}$, mass flow controller, GPM inlet, GPM outlet, small flask of oil. The outlet gas is vented to atmosphere through the production of bubbles in the oil (see Figure 8.13).

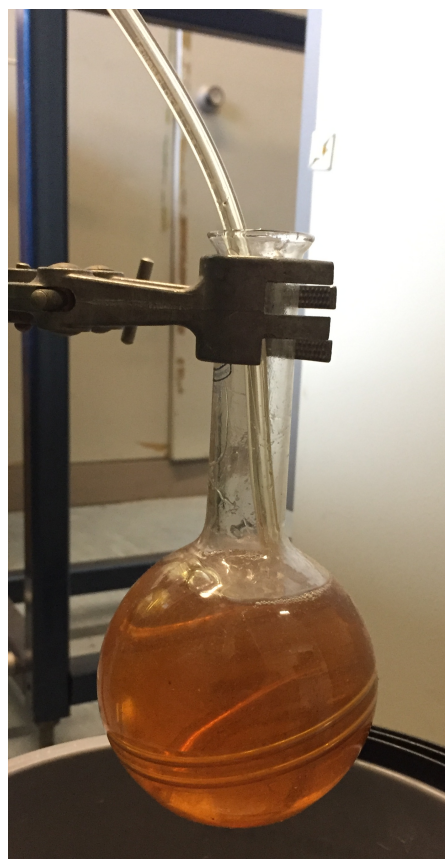

Fig. 8.13 Photo of the small flask filled with oil in which the GPM gas is vented. Two small bubbles can be seen floating to the oil surface indicating that gas is flowing through the GPM.

\begin{tabular}{|c|c|c|c|c|c|c|c|}
\hline $\begin{array}{l}\text { Head } \\
\mathrm{CH} 1\end{array}$ & $\begin{array}{c}\text { Acquisition } 1 \\
\mathrm{CH} 1\end{array}$ & $\begin{array}{l}\text { Head } \\
\mathrm{CH} 2\end{array}$ & $\begin{array}{c}\text { Acquisition } 1 \\
\mathrm{CH} 2\end{array}$ & $\begin{array}{c}\text { Head } \\
\mathrm{CH} 1\end{array}$ & $\begin{array}{c}\text { Acquisition } 2 \\
\mathrm{CH} 1\end{array}$ & $\begin{array}{l}\text { Head } \\
\mathrm{CH} 2\end{array}$ & Continued... \\
\hline
\end{tabular}

Fig. 8.14 Format of example binary data file with 2 channels of data and multiple waveform acquisitions. 
primary purpose of the experiment-specific software is to measure the pulse amplitudes and save the data as a ROOT-formatted [145] tree for straightforward high-level analysis.

Pulse amplitudes are measured by first calculating the baseline voltage and noise RMS from the first $30 \%$ of waveform samples. To optimise the software for speed and to account for the background noise of the waveform, the maximum value of the pulse is calculated as the mean voltage of the 11 waveform samples centred in time around the absolute maximum waveform value. Thus, the pulse amplitude simply becomes the pulse maximum minus the baseline voltage.

A software package was written to control the CAEN HV power supplies using a command line interface [207]. It uses the CAEN VME library to communicate with the hardware and set and read parameter values using the appropriate communication addresses. The program reads a voltage configuration from a user-inputted text file, sets the channel parameters accordingly, and throws a visible error marker in case of a problem. For example, if the detector experienced an electric breakdown and the power supply current spiked above the designated trip value, the program graphical output would switch from green to red and return a failure code upon exit.

To streamline the running of an experiment with the GPM, a script [208] was written to use the command line interfaces of all the previously described tools and run an experiment from start to finish. The script continuously ensures that an uninterrupted HV is supplied to the detector by controlling the CAEN power supplies, initiates and completes data acquisition by controlling the digitiser, performs data processing in the background using a second CPU core, and repeats the process for a specified series of detector voltage settings. With this script, the system is practically entirely automated.

\subsection{Detector Characterisation}

Several experiments were designed to test the functionality of the GPM in detecting LAr scintillation light, which would expand the range of operating conditions under which the detector was subjected. A test of the cryogenic functionality of the GPM was to measure the detector gain in different operating conditions to show the change in detector behaviour with temperature.

The gain of the GPM is determined from the single photoelectron collected charge distribution, which is well-modelled by a Polya distribution of the form [209]:

$$
P_{m}(g)=\frac{m^{m}}{\Gamma(m)} \frac{1}{G}\left(\frac{g}{G}\right)^{m-1} e^{-m \frac{g}{G}}
$$


where $m$ is a dimensionless real parameter, $g$ is the pulse charge, and $G$ is the detector gain. Relative gain comparisons can be performed by using the inverse slope of the linear part of the distribution, in log scale [210], which has a linear component of the form

$$
\log \left(P_{m}(g)\right) \approx-m \frac{g}{G}+\cdots .
$$

While the distributions here describe the single photoelectron collected charge events, given a sufficiently high quantum efficiency in the photocathode, it is possible for the detector to act as a proportional counter and measure multiple simultaneous photoelectron avalanches. This behaviour was shown in [185] for this detector.

For the goals of most of these experiments, imaging functionality of the detector was not used. Therefore, the anode and top strips were each read out from one end of the respective resistive lines, forcing all the pulsed current to two channels of the readout electronics, rather than the four required to produce $2 \mathrm{D}$ images.

\subsubsection{Experiment 1: Room Temperature Functionality}

The goal of the first experiment was to confirm the room temperature functionality of the GPM. The GPM was placed on the bench top (see Figure 8.15) and shielded from ambient light using a black cloth. The detector was biased based on the ideal scheme as determined in [185], which maximised the detector gain while minimising HV breakdowns on the THGEM components. The default voltages for this study were as delimited in Table 8.1, which were chosen to reduce breakdowns even further than in [185]. The UV light source used to create pulses in the detector was a small candle flame placed approximately $10 \mathrm{~cm}$ away from the window face of the detector. A sheet of plastic was placed between the fire and the GPM with a pinhole, to reduce the amount of light reaching the photocathode, allowing the detector to be run in single photoelectron mode. The multiplication gas $\left(\mathrm{CH}_{4}(5 \%) / \mathrm{Ne}\right)$ was flowed continuously through the detector at a rate of $10 \mathrm{~mL} / \mathrm{min}$ and vented to atmosphere.

Signals were measured from both sides of the resistive lines connecting the top and anode strips of the THCOBRA. Representative signals of preamplified pulses are shown in Figure 8.7. As expected, the anode signals were negative in polarity, and the top strip signals were positive in polarity. The pulses in Figure 8.7 have three distinct components: a fast rise time ( $\lesssim 1 \mu \mathrm{s}$ ), a slow rise time ( $2 \lesssim$ rise time $\lesssim 30 \mu \mathrm{s}$, depending on the $2 \mathrm{D}$ location of the avalanche in the detector), and a long decay. The fast rise time reflects the collection (or induction, in the case of the top strips) of charge on the electrode. The slow rise time is indicative of "ion backflow" [196], which occurs as the positive ions drift away from the 


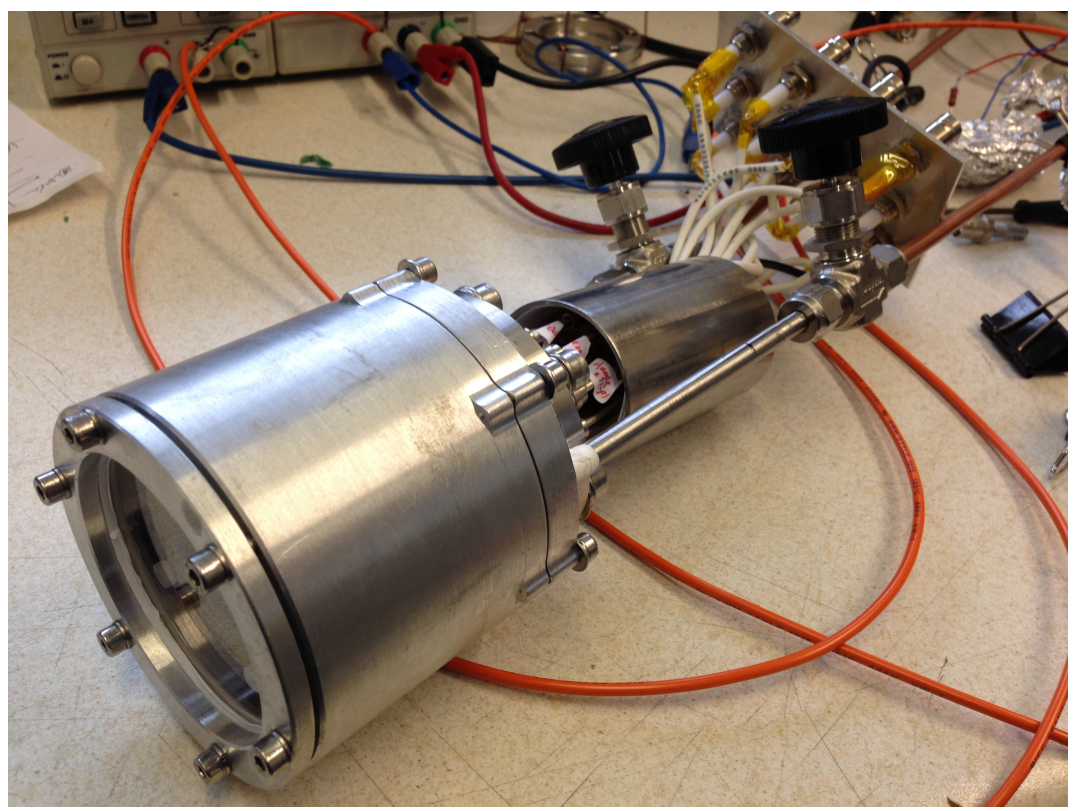

Fig. 8.15 Photo of GPM during room temperature test of functionality.

Table 8.1 Left: Default voltages applied to GPM components, relative to ground. Right: Effective component voltages.

\begin{tabular}{|c|c|c|c|}
\hline$V_{\text {Grid }}$ & $-685 \mathrm{~V}$ & \multirow{2}{*}{$\Delta V_{\text {Extract }}$} & \multirow{2}{*}{$0 \mathrm{~V}$} \\
\hline \multirow[b]{2}{*}{$V_{\text {Top } 1}$} & \multirow[b]{2}{*}{$-685 \mathrm{~V}$} & & \\
\hline & & & \\
\hline \multirow{2}{*}{$V_{\text {Bottom } 1}$} & \multirow{2}{*}{$-90 \mathrm{~V}$} & $\Delta V$ THGEM 1 & $390 \mathrm{v}$ \\
\hline & & $\Delta V_{\text {Transfer } 1}$ & $90 \mathrm{~V}$ \\
\hline$V_{\text {Top } 2}$ & $0 \mathrm{~V}$ & $\Delta \mathrm{I}$ & $550 \mathrm{~V}$ \\
\hline \multirow{2}{*}{$V_{\text {Bottom } 2}$} & \multirow{2}{*}{$550 \mathrm{~V}$} & VIHGEMI 2 & \\
\hline & & $\Delta V_{\text {Transfer } 2}$ & $90 \mathrm{~V}$ \\
\hline$V_{\text {Top Strips }}$ & $640 \mathrm{~V}$ & $\Delta V_{\text {mugnos }}$ & $460 \mathrm{~V}$ \\
\hline \multirow[t]{2}{*}{$V_{\text {Cathode }}$} & \multirow[t]{2}{*}{$1100 \mathrm{~V}$} & COBRA & \\
\hline & & $\Delta V_{\text {Anode-Cathode }}$ & $125 \mathrm{~V}$ \\
\hline$V_{\text {Anode }}$ & $1225 \mathrm{~V}$ & $\boldsymbol{\Lambda}$ & $125 \mathrm{~V}$ \\
\hline$V_{\text {Plane }}$ & $1100 \mathrm{~V}$ & $\Delta V$ Plane-Anode & $-125 \mathrm{v}$ \\
\hline
\end{tabular}


readout electrodes inducing additional currents. The long decay time is caused by the slow discharge of the feedback capacitor in the charge sensitive preamplifier.

It was also discovered during this test that the particular shaping time $(8 \mu \mathrm{s})$ of the charge amplifiers caused them to integrate not only the fast electron-induced current pulses $(<1 \mu \mathrm{s})$ but also the currents induced by the positive ions moving away from the electrodes (1-20 $\mu \mathrm{s})$. This is observed by the continuing increase of voltages in most channels after $1 \mu$ s following the start of the pulse in Figure 8.7. The long integration time resulted in an incorrect measurement of the actual collected charge of the avalanche. Thus, the gain and the measured energy reconstruction were skewed upwards by an amount which is dependent on the level of ion backflow in the GPM.

While the charge multiplication stages of the GPM were confirmed to be completely functional, the status of the CsI photocathode was still unknown. The wide-band light wavelength spectrum produced by the flame contained both very high and very low energy photons, which have the potential to eject electrons via the photoelectric effect from many materials in the GPM, such as copper, steel, CsI, or even the PCB itself. Further study is required using LAr scintillation light directly and a positive control group such as a PMT.

The main conclusion from Experiment 1 is that the GPM successfully and consistently multiplied photoelectrons, and produced measurable signals. However, it is still unknown if the GPM is sensitive to VUV light, or if it functions at cryogenic temperatures.

\subsubsection{Experiment 2: Warm/Cold Gain Comparison}

The second experiment using the GPM was to test how the gas gain is affected by a significant reduction in operating temperature. Previous experiments [193] have shown that there is a significant gain reduction when operating in LXe $(165 \mathrm{~K})$, which is caused by an increase in the gas density. This increases the rate of electron collisions in the gas, hindering the acceleration, and hence, reducing the secondary ionisation in an avalanche. One way to mitigate this loss of gain and encourage the detector to produce increased electron multiplication is to raise the operating voltages of the THGEMs.

For this test, the GPM was placed in a thermally insulated bucket, so that the detector body was submerged in solid chips of dry ice $\left(\mathrm{CO}_{2}\right)$ for an extended period. A $355 \mathrm{~nm}$ LED was placed $5 \mathrm{~cm}$ from the GPM window and was powered using a range of currents to produce varying light intensities, as in Figure 8.16. The same front end electronics and analysis method were used as in Experiment 1, including the $8 \mu$ s shaping amplifiers. The multiplication gas was flowed continuously, venting to atmosphere, at a rate of $10 \mathrm{~mL} / \mathrm{min}$. The GPM HV was set up using the scheme in Table 8.2, where any arbitrary voltage, $\Delta V$, could be added to the three THGEM stages denoted on the corresponding rows. 


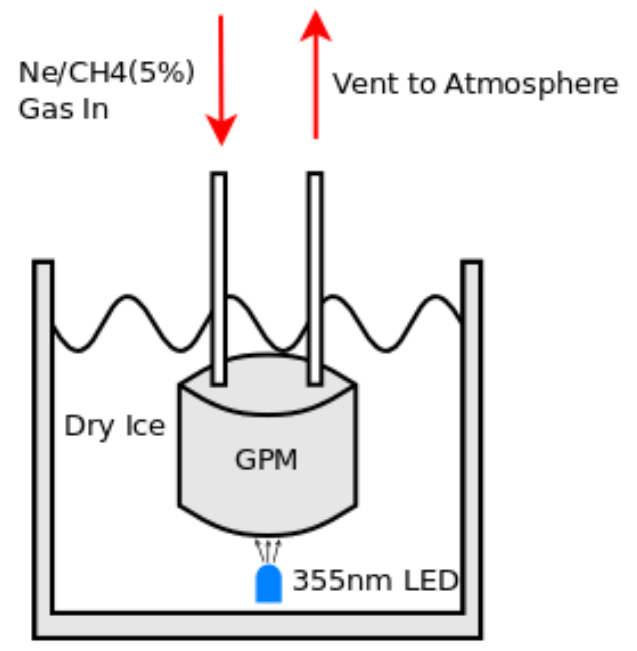

Fig. 8.16 Experiment 2 setup of GPM and LED in insulated bucket, submerged in dry ice chips.

Table 8.2 GPM HV scanning scheme for gain measurements.

\begin{tabular}{|l|l|l|}
\hline$\Delta V_{\text {Extract }}$ & $0 \mathrm{~V}$ & \\
\hline$\Delta V_{\text {THGEM 1 }}$ & $550 \mathrm{~V}$ & $+\Delta V$ \\
\hline$\Delta V_{\text {Transfer 1 }}$ & $90 \mathrm{~V}$ & \\
\hline$\Delta V_{\text {THGEM 2 }}$ & $550 \mathrm{~V}$ & $+\Delta V$ \\
\hline$\Delta V_{\text {Transfer 2 }}$ & $90 \mathrm{~V}$ & \\
\hline$\Delta V_{\text {THCOBRA }}$ & $500 \mathrm{~V}$ & $+\Delta V$ \\
\hline$\Delta V_{\text {Anode-Cathode }}$ & $125 \mathrm{~V}$ & \\
\hline$\Delta V_{\text {Plane-Anode }}$ & $-125 \mathrm{~V}$ & \\
\hline
\end{tabular}


At room temperature, before dry ice was added to the bucket, the GPM was run at different operating voltages, with $\Delta V$ ranging from $-30 \mathrm{~V}$ to $+30 \mathrm{~V}$ in $5 \mathrm{~V}$ increments. An example of the measured pulse height distribution from the anode and top strips is shown in Figure 8.17. For all steps of $\Delta V$, the inverse slope of the exponential fit to the linear part of the distribution was converted to an absolute gain using the calibrated conversion constant in Figure 8.11.

The process above was repeated when the detector was cooled down to $195 \mathrm{~K}$ using chips of solid $\mathrm{CO}_{2}$. Due to the increased gas density inside the GPM volume, the gain was reduced, and higher voltages were applied to increase the gain into the measurable region for the same data acquisition parameters. Pulse height distributions were recorded for $\Delta V$ ranging from $+50 \mathrm{~V}$ to $+90 \mathrm{~V}$ in $10 \mathrm{~V}$ increments, and the detector gains calculated. Higher voltages were not applied due to the appearance of HV breakdowns in the THGEM components above $\Delta V \geq+100 \mathrm{~V}$.

Comparison of the GPM gains at room temperature and in dry ice is shown in Figure 8.18. As expected, at a constant temperature, increasing the THGEM voltage increased the overall GPM gain as the electrons had higher accelerations in the larger electric fields. The absolute gains reported here suffer from systematic errors due to the long electronic shaping time of the Cremat CR-200-8 $\mu$ s shaping amplifiers. In general, the output pulse amplitudes were overestimated due to the influence of ion backflow which increased the apparent current flow in the THCOBRA components. This overestimation of charge is a possible systematic error in that it would increase the upper tail of the Polya distributions and skew the observed gains upward.

In this experiment, the GPM was showed to function at a cryogenic temperature. As expected, the measured detector gain decreased due to the increased gas density at the low temperature, but high gains were recovered by increasing the applied electric fields across the THGEM components.

\subsubsection{Experiment 3: Argon Scintillation Light Measurement}

The third experiment was to test directly the ability of the GPM to detect argon scintillation light and, hence, to test the functionality of the CsI photocathode. This test was completed by observing alpha particle-induced scintillation in argon gas with the GPM and a PMT as the control. The source used was $1 \mu \mathrm{Ci}$ of ${ }^{241} \mathrm{Am}$ which produced $5.5 \mathrm{MeV}$ alpha particles.

This test was completed in the main Dewar volume of the Sheffield LAr test stand (Chapter 7), which was filled with 1 atmosphere of either argon or nitrogen gas at room temperature during operation. As shown in Figure 8.19, a PTFE mount was constructed to hold in place the GPM and PMT with the sensitive areas of each detector facing the same volume of gas below, where the ${ }^{241}$ Am source is located. The PMT used in this test 

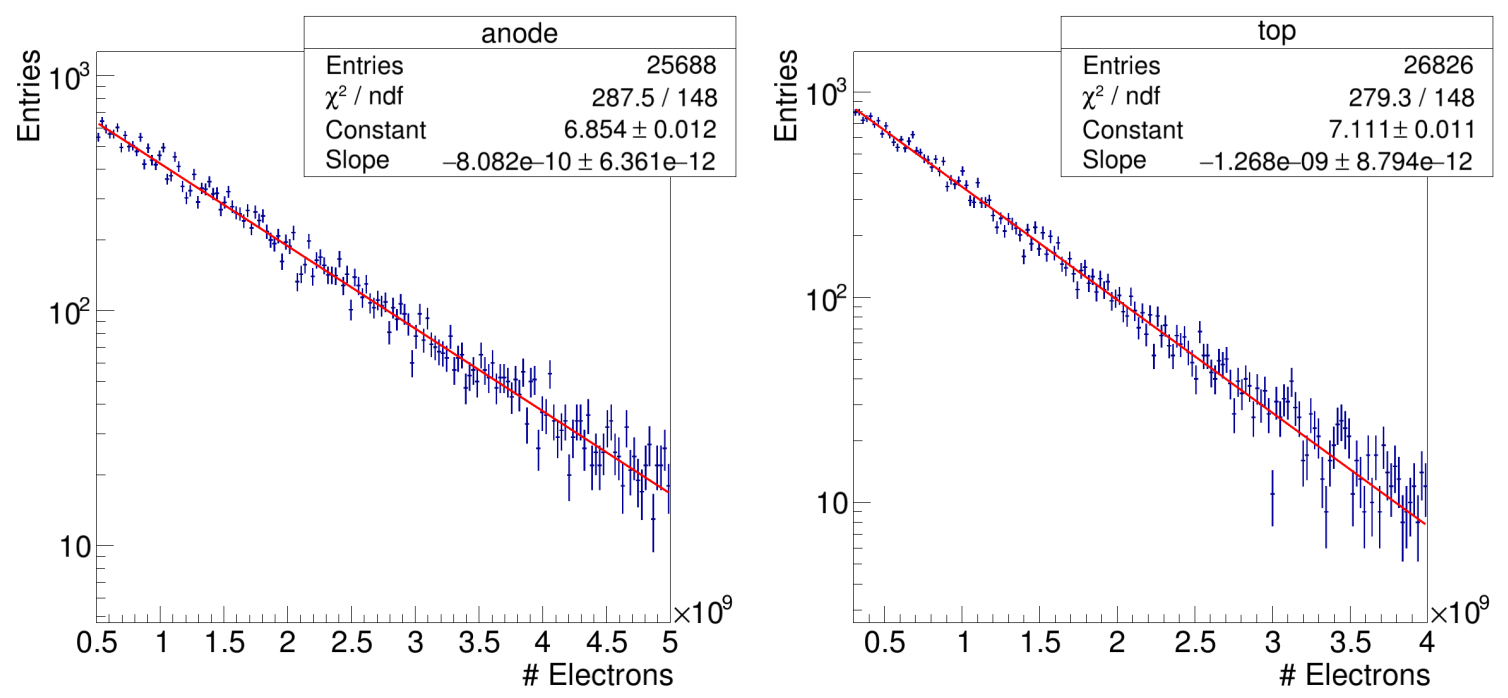

Fig. 8.17 Pulse height distribution for anode (left) and top (right) strips. Room temperature operation, with operating voltages as in Table 8.2 with $\Delta V=-10 \mathrm{~V}$.

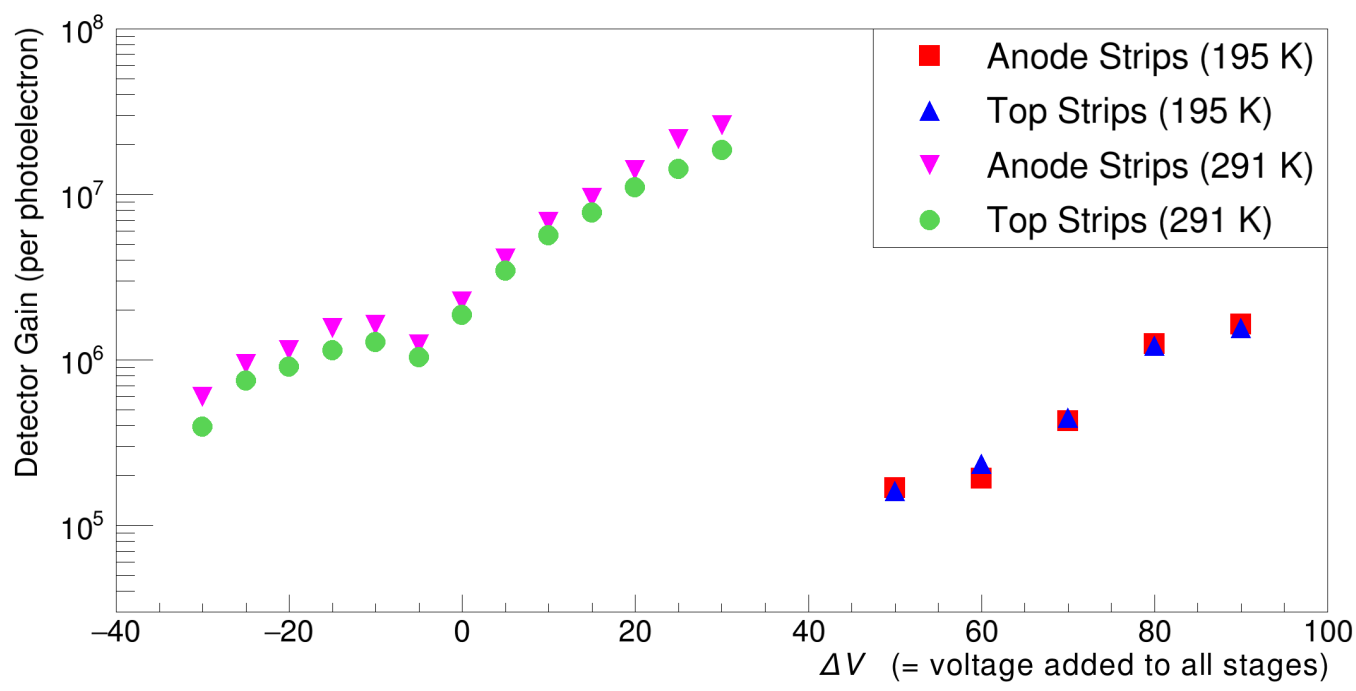

Fig. 8.18 GPM absolute gain measurements at room temperature and in dry ice. $\Delta V$ represents the voltages added to the THGEM and THCOBRA components, in addition to the default values in Table 8.2. 
is a Hamamatsu R11065 low background cryogenic-ready tube. The PMT was negatively biased at $-1500 \mathrm{~V}$, and the raw pulses shaped using a Tennelec TC 241 amplifier with $1 \mu \mathrm{s}$ shaping time. This particular PMT model and the base circuit was used in the DarkSide experiment [42] to measure LAr scintillation light. For the PMT to be sensitive to LAr scintillation, the active volume of gas in the test stand was lined with a cylindrical sheet of thin PTFE on which was sprayed a coating of TPB for wavelength shifting. For this test, the GPM was biased according to Table 8.1 and was supplied with a gas flow rate of $10 \mathrm{~mL} / \mathrm{min}$.

In this experiment, the test stand was initially filled with nitrogen gas, which has a low scintillation light yield of 140 photons per $1 \mathrm{MeV}$ of energy loss. However, for gaseous argon, the yield is $O\left(10^{4}\right)$ photons per $1 \mathrm{MeV}$ of energy loss [211,212]. Therefore, including the wavelength shifting effects of the TPB, the PMT was expected to be sensitive to argon and nitrogen scintillation, albeit at much different count rates. Because the scintillation light yield in nitrogen is two orders of magnitude lower than in argon, there was a noticeable reduction in the trigger rate and obvious differences in the pulse height spectrum as the nitrogen scintillation signal was dominated by background thermal noise.

Figure 8.20 shows the spectrum of pulse amplitudes from the PMT in nitrogen gas normalised by the total runtime to give the pulse rate. After flushing the nitrogen from the test stand and filling with argon gas, the test was repeated, revealing the pulse amplitude distribution for argon in Figure 8.21. In both cases, the single photoelectron signal is seen on the left side of the spectra. However, the argon spectrum has a significant feature which is not present when the detector was in nitrogen. The multiple photoelectron feature around 40 ADC in the argon spectrum was attributed to argon scintillation light and was confirmed by the shape of the pulses in this population. Figure 8.22 shows an example of such a pulse, which was consistent with the expected shape and time of alpha scintillation events in LAr [213]. LAr scintillation light is abundant in the Dewar when filled with argon gas.

The same test was performed with the GPM in the test stand. In nitrogen gas, the uncalibrated pulse height spectrum (Figure 8.23) showed no distinguishable features apart from the single photoelectron upper tail of the Polya distribution, as in Figure 8.17. The time between triggered pulses in the GPM, $\Delta T$, is a Poisson process as the detected events are random at a constant average rate, so the distribution is an exponential with a characteristic lifetime which is related to the trigger rate. In nitrogen, this distribution is shown in Figure 8.25, which has an exponential lifetime of $0.74 \mathrm{~s}$.

In argon gas, if the CsI photocathode had a sufficiently high quantum efficiency and was not degraded, the DAQ trigger rate will be significantly higher as the alpha source scintillates the gas releasing large numbers of VUV photons. The distribution of times between DAQ triggers of the GPM in argon is shown in Figure 8.26, which shows a slight increase in the 


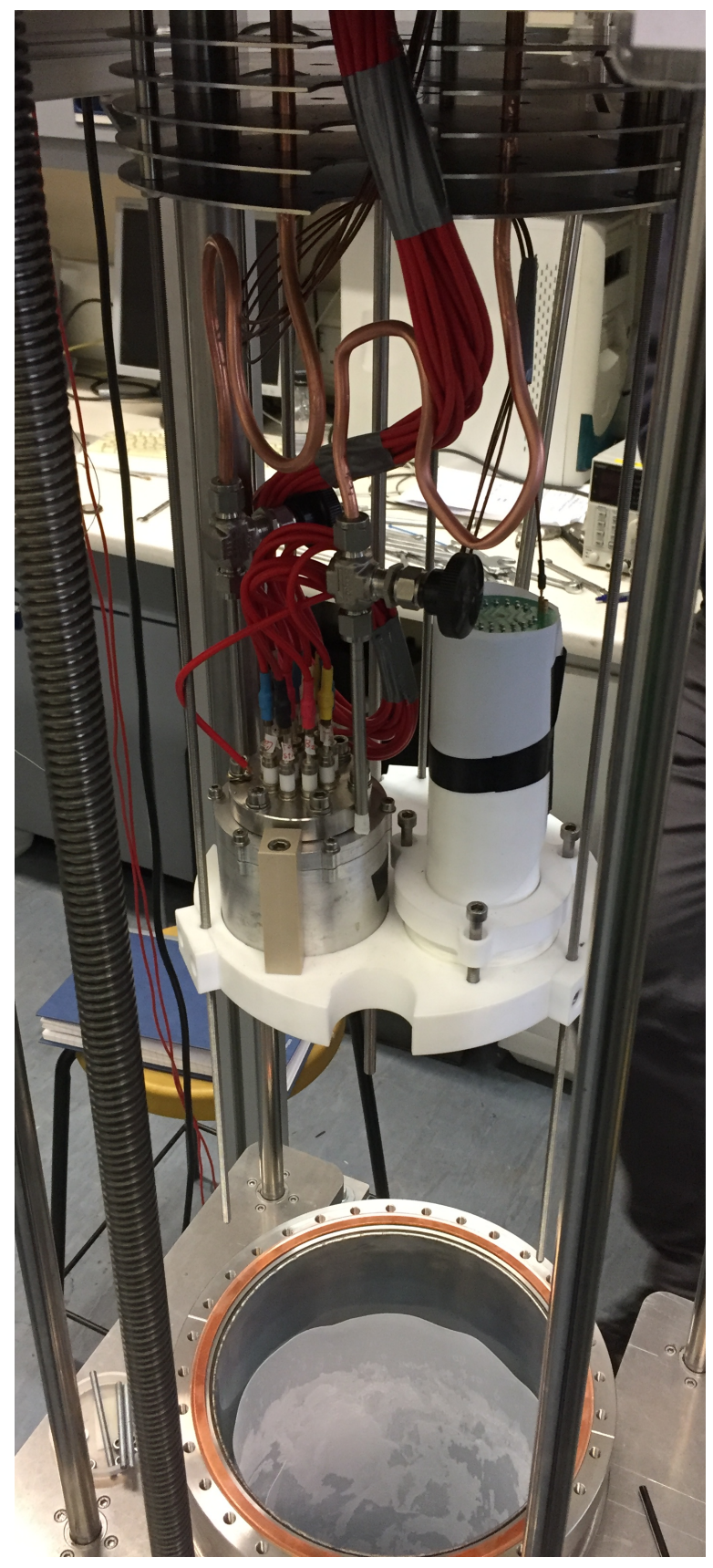

Fig. 8.19 GPM (left) and PMT (right) experiment setup in the test stand before the Dewar was closed. Detectors were supported by PTFE mount which allows the sensitive areas of the detectors to be exposed to the same experiment volume. White sheet of PTFE is also shown inside the Dewar, roughly coated with TPB. 


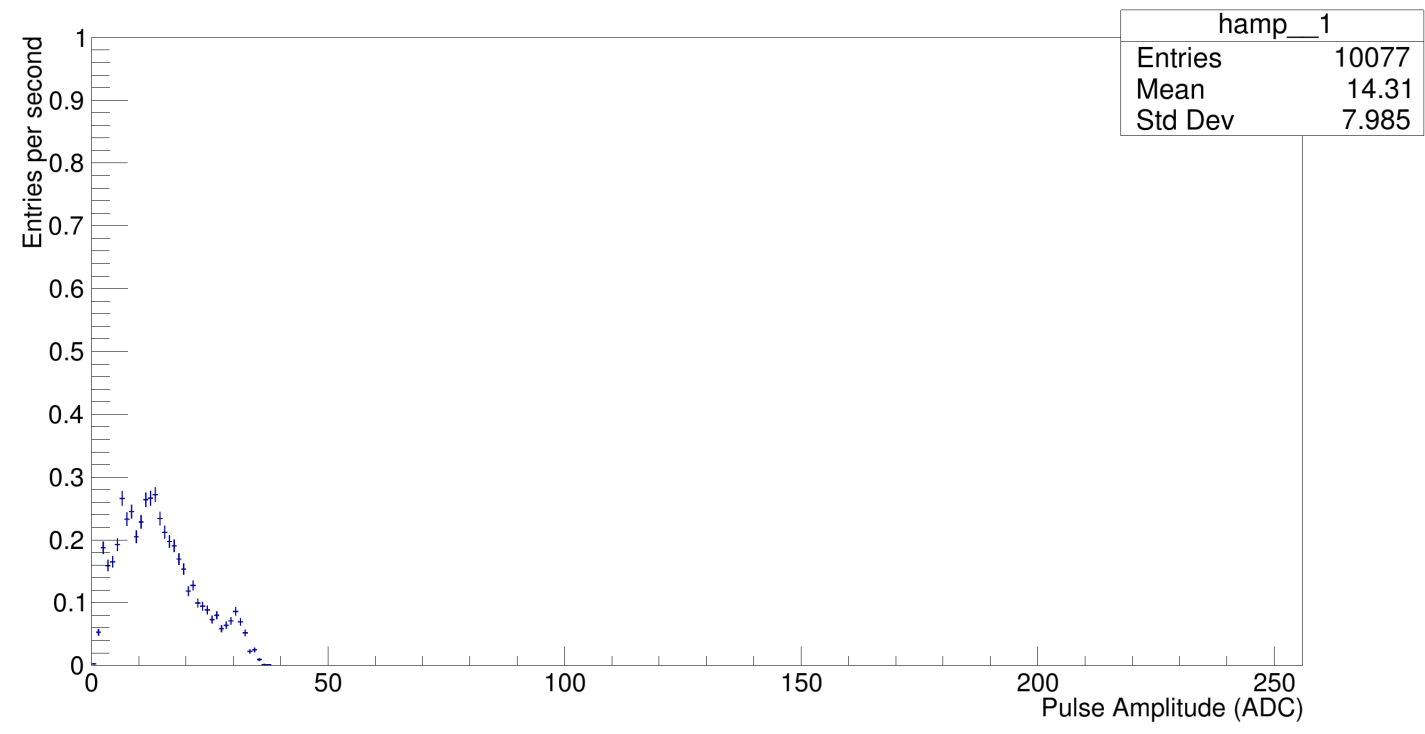

Fig. 8.20 PMT+TPB in nitrogen gas. Pulse amplitude distribution scaled by total run time (2040 seconds) to acquire 10k pulses.

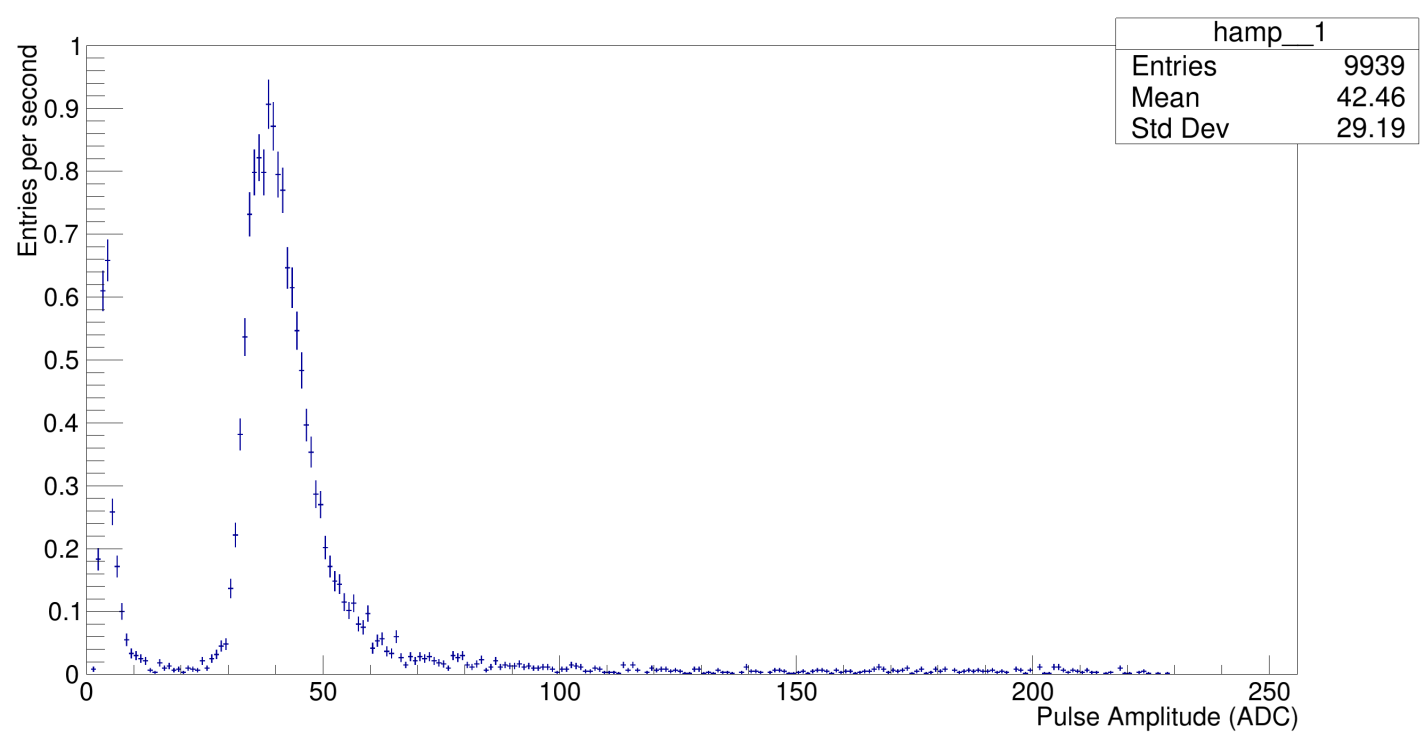

Fig. 8.21 PMT+TPB in argon gas. Pulse amplitude distribution scaled by total run time (596 seconds) to acquire $10 \mathrm{k}$ pulses. 

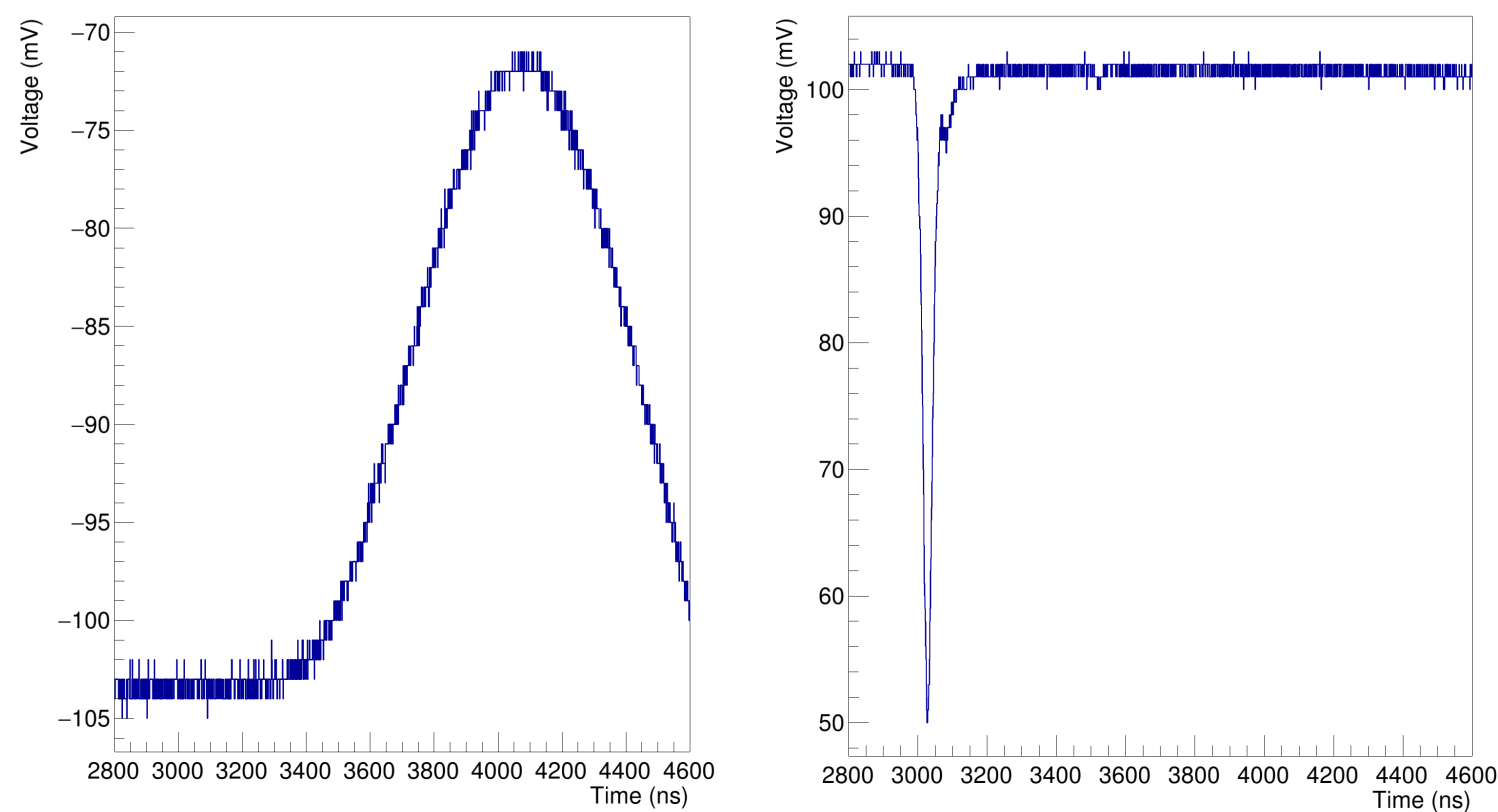

Fig. 8.22 Right: Argon scintillation raw PMT pulse. Left: Argon scintillation PMT pulse after shaping.

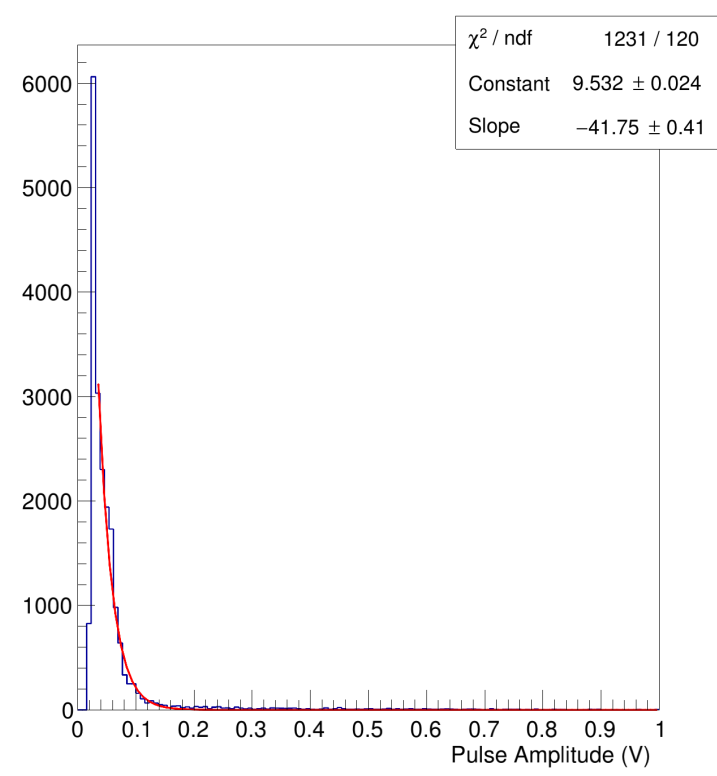

Fig. 8.23 Pulse amplitude distribution of GPM in nitrogen gas at room temperature. Manual coarse gain (10x) on Cremat shaping amplifier switched $o f f$.

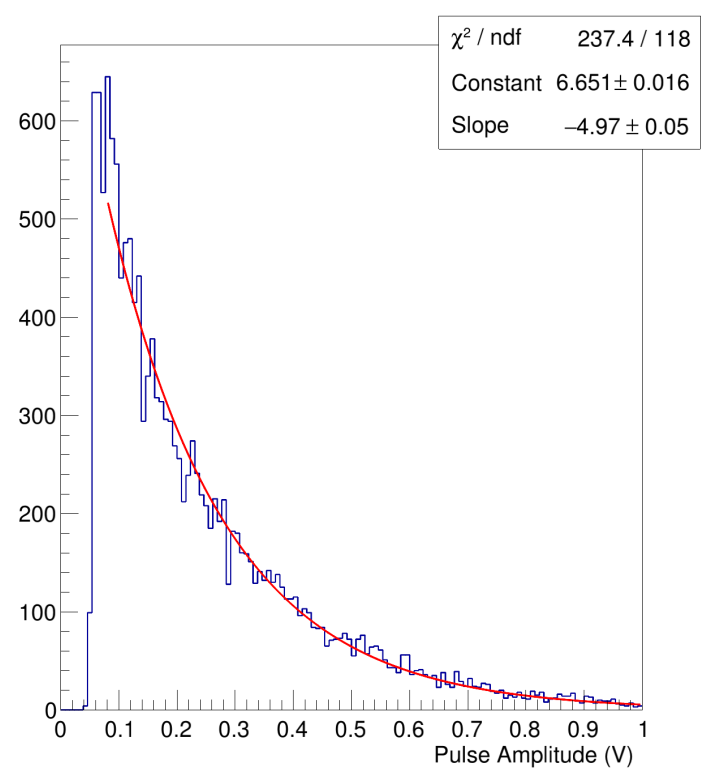

Fig. 8.24 Pulse amplitude distribution of GPM in argon gas at room temperature. Manual coarse gain (10x) on Cremat shaping amplifier switched $o$. 


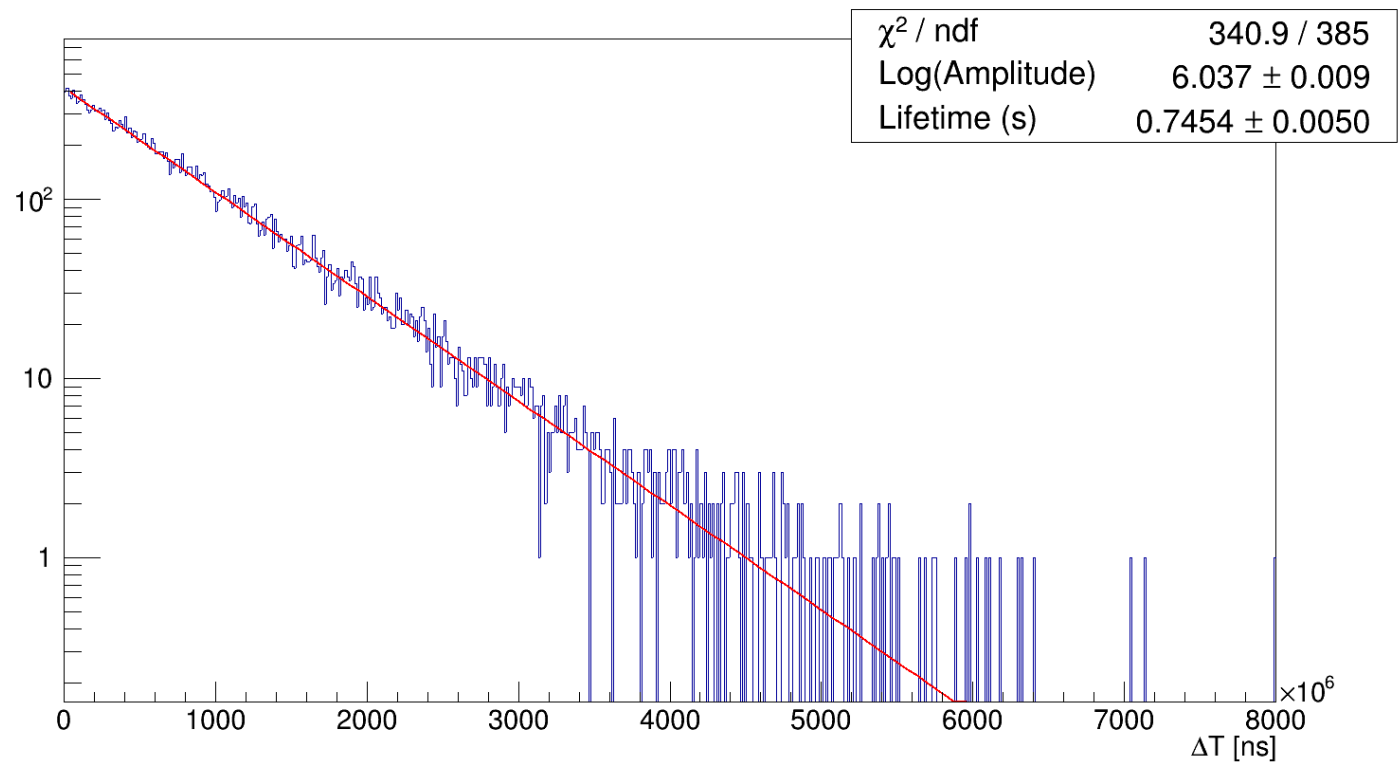

Fig. 8.25 Distribution of time between triggers in the GPM while in nitrogen gas. The lifetime of the exponential is related to the average $\Delta T$.

pulse rate from the same measurement in nitrogen ( $0.71 \mathrm{~s}$ lifetime). This increase in pulse rate is likely due to random statistical fluctuations or slight shifts in the triggering threshold or signal baseline and not an actual physical phenomenon. The pulse height distribution of the GPM in argon also did not show any distinct multiple photoelectron peaks, or any difference from the distribution when the GPM was in nitrogen, apart from setting a manual 10x coarse gain switch (see Figure 8.24). The GPM did not observe argon scintillation light.

Because the PMT observed argon scintillation light and the GPM did not, a fundamental issue with the GPM is implied. The most likely possibility is that the CsI photocathode is significantly degraded such that the GPM is no longer sensitive to VUV photons. The degradation is due to moisture leaking into the GPM volume and causing hydrolysis of the CsI, despite storing the GPM for long periods of time in a dry environment (i.e. sealed and filled with a slight overpressure of $\left.\mathrm{CH}_{4}(5 \%) / \mathrm{Ne}\right)$. For the GPM to function as intended, a new CsI coating must be evaporated, and any leaks in the detector must be sealed, but this is the subject of future work.

This experiment showed that the GPM was not sensitive to VUV light from argon scintillation. It is anticipated, however, that by evaporating a new CsI photocathode, the VUV sensitivity of the detector will be restored. 


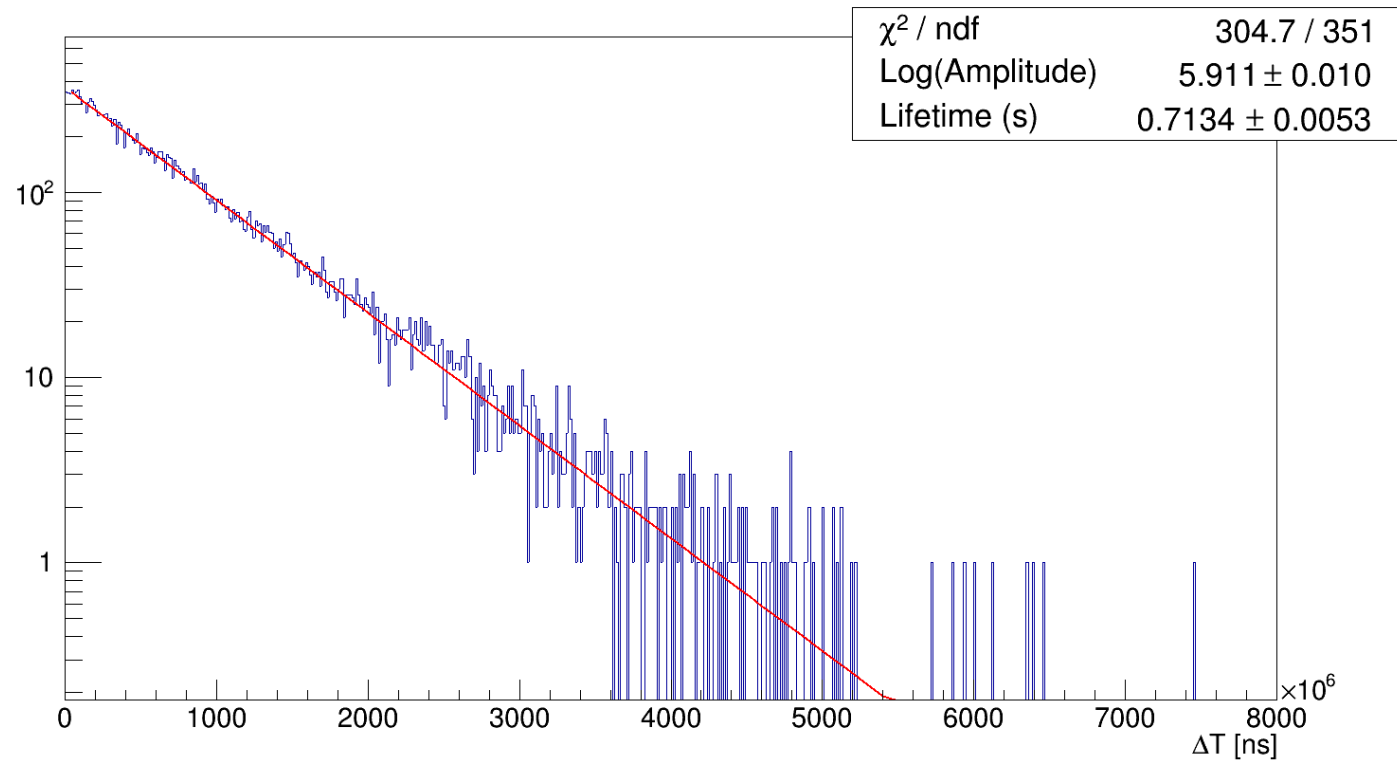

Fig. 8.26 Distribution of time between triggers in the GPM while in argon gas. The lifetime of the exponential is related to the average $\Delta T$.

\subsubsection{Experiment 4: 77 K Operation}

Given that the CsI photocathode in the GPM was degraded beyond the point of functionality, the goals of the final experiment were adjusted from directly observing LAr scintillation light to analysing the performance change of the detector when cooled down to LAr temperature. This last test was conducted while submerging the GPM in $\mathrm{LN}_{2}$, which has a similar boiling point to LAr. This experiment was set up with the GPM installed in the Sheffield LAr test stand, as before, but using the $355 \mathrm{~nm}$ LED as the source of light rather than the ${ }^{241} \mathrm{Am}$ source. Also, the PMT was not used as the goal was no longer to detect argon scintillation light directly.

In this experiment, the LAr test stand main Dewar volume was filled with $\mathrm{LN}_{2}$, the GPM with high multiplication gas flow $(100 \mathrm{~mL} / \mathrm{min})$, and biased according to the scheme in Table 8.2. About 30 minutes following initial cooldown and submersion in $\mathrm{LN}_{2}$, it was observed that the multiplication gas flow slowed and stopped despite the mass flow controller attempting to provide the preset flow rate to the GPM. The blockage was seen when the mass flow controller monitor showed a drop of flow rate to $0 \mathrm{~mL} / \mathrm{min}$ as pressure built up in the pipes. However, gas bubbles were still being produced in the oil flask. Either the $\mathrm{LN}_{2}$ leaked into and filled the GPM volume, or the methane froze out and blocked the pipes (essentially causing a "heart attack"). 
The first possibility implies a problem with the construction of the detector such as incorrectly matched coefficients of thermal expansion (CTE) in the joining of two different materials. For instance, the CTE of Teflon is about ten times greater than aluminium [214], possibly introducing a problem with the $\mathrm{MgF}_{2}$ window seal on the GPM. The second possibility implies a fundamental problem with the choice of multiplication gas in the GPM. The freezing point of methane $(91 \mathrm{~K})$ is above the boiling point of both nitrogen $(77 \mathrm{~K})$ and argon $(87 \mathrm{~K})$, which caused solid condensation on all surfaces inside the GPM. Besides possibly causing a blockage, this condensation can affect the performance of the GPM as a whole in that it can affect the electric fields, and hence, the gain of the detector. Whatever caused the gas flow blockage, two problems in the GPM design were exposed and will need to be fixed for the experiment to continue.

Before the blockage occurred, the GPM was functional and provided single photoelectron pulses consistent with what was seen in previous experiments (Figure 8.27). Also, due to the low temperature, $\Delta V$ of $+100 \mathrm{~V}$ was used for the GPM to produce measurable pulses using the same DAQ parameters. However, due to the short time in which the detector was functional, detailed studies of the gain were not possible. Nevertheless, the GPM's sensitivity to the $355 \mathrm{~nm}$ LED was tested at $77 \mathrm{~K}$, which showed a significant increase in the rate and amplitude of pulses (Figure 8.28).

Even though the LED photon energy is lower than the work function of CsI and Copper, the GPM was sensitive to the intense light, despite the significantly reduced quantum efficiency. The GPM was functional at $77 \mathrm{~K}$, despite the reduced gain, and responsive to $355 \mathrm{~nm}$ light from an LED.

\subsection{Summary}

The electron multiplication properties of the GPM were demonstrated at room temperature down to $77 \mathrm{~K}$. The relationship between the THGEM electric fields and detector gain was exhibited at several operating temperatures. Also, the GPM was shown to be sensitive to light which is outside of the range of wavelength sensitivity of the photocathode given a sufficiently intense source of illumination. Qualitatively, these results are consistent with other studies involving GEM-based detectors operating at cryogenic temperature [215].

Despite the successes, there were several operational and construction problems which interfered with the successful completion of all initial experimental goals. Therefore, future work is needed to ensure the robustness of the detector and housing, to reevaluate the choice of multiplication gas in the detector (for example, $\mathrm{He}+\mathrm{H}_{2}$ and $\mathrm{Ne}+\mathrm{H}_{2}$ have been shown to produce high gains in GEM detectors at $77 \mathrm{~K}$ [216]), and to reevaporate the photocathode to 


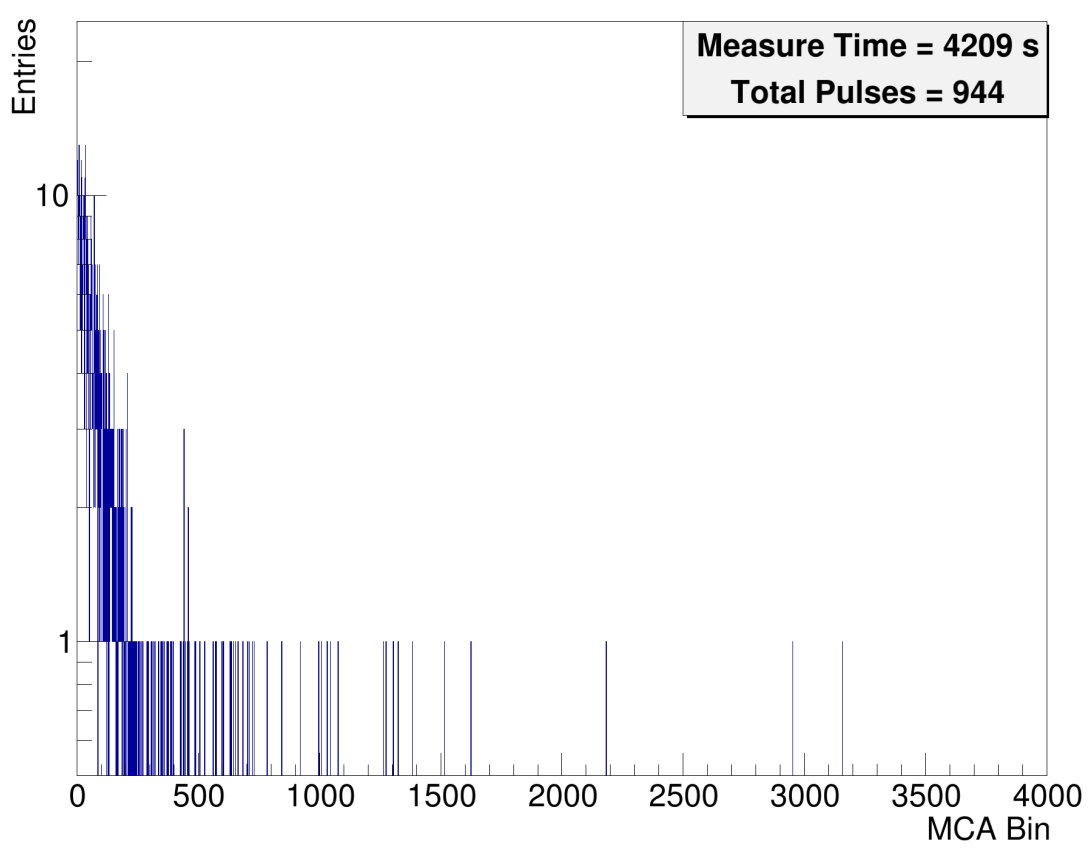

Fig. 8.27 Single photoelectron pulse height distribution of GPM in $\mathrm{LN}_{2}$ and $\Delta V=+100 \mathrm{~V}$. No LED.

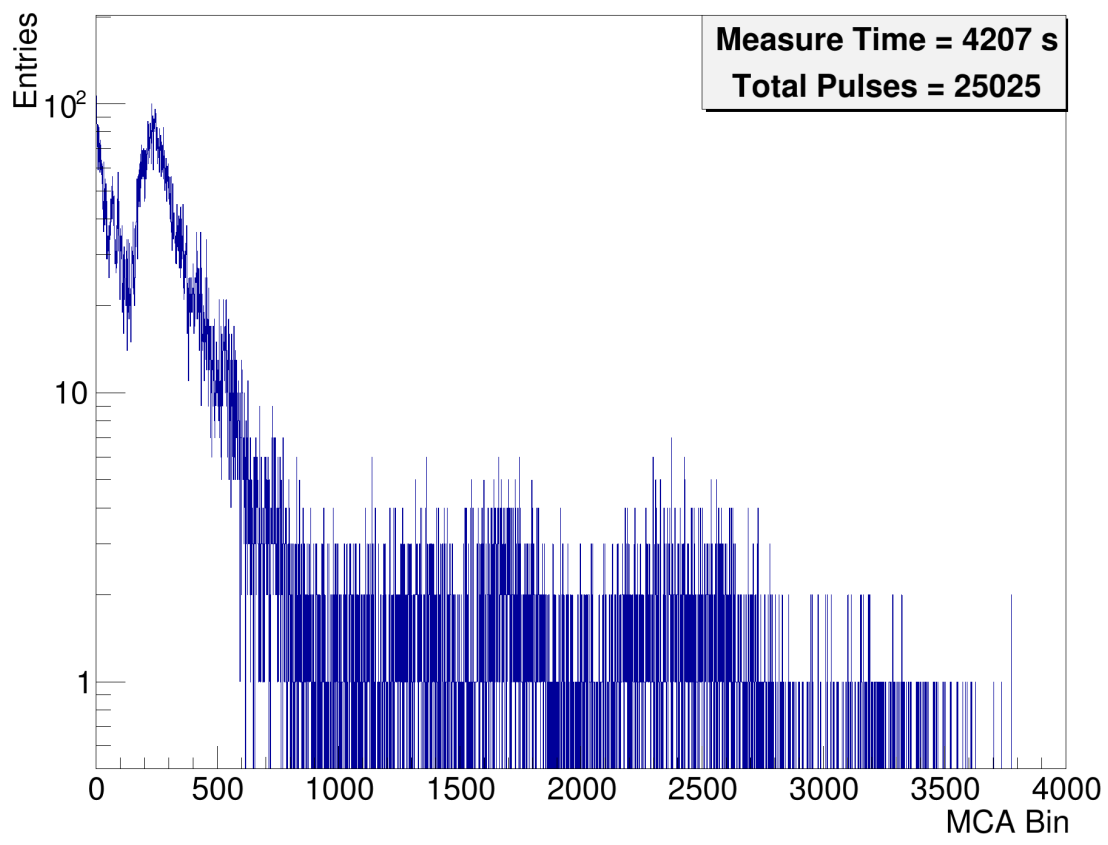

Fig. 8.28 Single photoelectron pulse height distribution of GPM in $\mathrm{LN}_{2}$ and $\Delta V=+100 \mathrm{~V}$. With $355 \mathrm{~nm}$ LED light. 
demonstrate the GPM sensitivity to LAr scintillation light. The GPM has the potential to be a powerful general purpose position-sensitive VUV photodetector for both room temperature and LAr applications. 



\section{Chapter 9}

\section{Conclusion}

The work in this thesis covers a wide range of topics, connected by the overall motivation to design, build, and operate a massive scale LAr TPC experiment for neutrino oscillation and other rare physics detection. Advances have been made in many areas, including analysis of extremely noisy data from the DUNE 35-ton prototype detector, the successful design and operation of a system of cryogenic cameras, the design and construction of a recirculating LAr test stand, and cryogenic tests of a GPM for LAr scintillation light detection.

In section 3.5, a vital detector calibration study was completed where the relative coordinate system of two separate detector sub-systems was determined using TPC data only. The muon counter $z$ coordinates were found to be consistent with the TPC $z$ coordinates, to within a few centimetres, well within tolerance. Future LAr TPC experiments, in particular, SBND, can use this analysis method since they will use a very similar detector setup with muon counters surrounding the cryostat.

Chapter 4 described the development and operation of a system of cryogenic cameras for HV monitoring of the DUNE 35-ton cryostat. The system was operational for approximately 50 days during the entire Phase II run, where some image degradation was observed, but no camera failures occurred. No HV breakdowns in the cryostat were recorded because none happened in the field of view of any of the cameras. Nevertheless, the system was deemed successful, and the general idea is currently being pursued by other LAr TPC experiments, including ProtoDUNE. So far, it is the only array of cameras capable of monitoring a large LAr cryostat from within without additional heating, despite several known attempts.

The reconstruction of data and analysis of electron lifetime in the 35-ton TPC was presented in chapters 5 and 6. Despite the excessively high electronic noise, the measurement of the lifetime was still completed with reasonable uncertainties. It is consistent with other direct measurements of the argon electron lifetime, taking into consideration inefficient mixing of impurities throughout the cryostat. The high noise which plagued the TPC data 
caused systematic uncertainties on the measurement to be outside the acceptable range for future sensitive physics experiments, such as DUNE. Still, the analysis framework created is powerful in reconstructing data with a very low $\mathrm{S} / \mathrm{N}$, possibly allowing very low energy event reconstruction.

Chapter 7 described the design, construction, and operation of the Sheffield LAr test stand. The purpose of the test stand is to provide a general purpose high purity vessel for conducting long-term tests of various detectors or physical principles. It was successfully commissioned in June 2016.

In chapter 8, the GPM was presented along with the custom developed data acquisition system. Experiments to test the detector operation in LAr successfully observed light signals while operating at $77 \mathrm{~K}$. On the other hand, experiments to prove the VUV sensitivity of the GPM were inconclusive as construction problems were discovered. There is much future work to be done on the GPM, but it has the potential to be a very powerful detector for LAr scintillation light, which is a recognised problem in the design and construction of DUNE.

The field of neutrino physics has an exciting future with the construction and operation of kiloton-scale LAr TPCs. The width and breadth of work required to pull off these experiments successfully are huge, and the work presented here, however insignificant, has advanced the already massive body of research toward this goal. One can only hope to still be in the field when first neutrinos are detected in DUNE. 


\section{References}

[1] W. Pauli, "Letter to L. Meitner and colleagues." (Open letter to the participants of the conference at Tubingen), 1930.

[2] F. Reines and C. L. Cowan, Detection of the free neutrino, Phys. Rev. 92 (Nov, 1953) 830-831.

[3] R. Davis, D. S. Harmer and K. C. Hoffman, Search for neutrinos from the sun, Phys. Rev. Lett. 20 (May, 1968) 1205-1209.

[4] J. N. Bahcall, N. A. Bahcall and G. Shaviv, Present status of the theoretical predictions for the ${ }^{37} \mathrm{Cl}$ solar-neutrino experiment, Phys. Rev. Lett. 20 (May, 1968) 1209-1212.

[5] V. Gribov and B. Pontecorvo, Neutrino astronomy and lepton charge, Phys. Lett. B 28 (1969) $493-496$.

[6] Z. Maki, M. Nakagawa and S. Sakata, Remarks on the unified model of elementary particles, Prog. Theor. Phys. 28 (1962) 870-880.

[7] SNO collaboration, Q. R. Ahmad, R. C. Allen, T. C. Andersen, J. D.Anglin, J. C. Barton, E. W. Beier et al., Direct evidence for neutrino flavor transformation from neutral-current interactions in the Sudbury Neutrino Observatory, Phys. Rev. Lett. 89 (Jun, 2002) 011301.

[8] Particle Data Group collaboration, C. Patrignani et al., Review of Particle Physics, Chin. Phys. C40 (2016) 100001.

[9] B. Pontecorvo, Neutrino experiments and the problem of conservation of leptonic charge, Sov. Phys. JETP 26 (1968) 984-988.

[10] B. Pontecorvo, Inverse beta processes and nonconservation of lepton charge, Sov. Phys. JETP 7 (1958) 172-173.

[11] K. Eitel, Direct neutrino mass experiments, Nucl. Phys. Proc. Suppl. 143 (2005) 197-204.

[12] L. Wolfenstein, Neutrino oscillations in matter, Phys. Rev. D 17 (May, 1978) 2369-2374.

[13] S. P. Mikheyev and A. Y. Smirnov, Resonant amplification of $v$ oscillations in matter and solar-neutrino spectroscopy, Il Nuovo Cimento C 9 (Jan, 1986) 17-26. 
[14] F. Capozzi, E. Lisi, A. Marrone, D. Montanino and A. Palazzo, Neutrino masses and mixings: Status of known and unknown $3 v$ parameters, Nucl. Phys. $\mathbf{B 9 0 8}$ (2016) 218-234, [1601.07777].

[15] J. D. Vergados, H. Ejiri and F. Šimkovic, Theory of neutrinoless double-beta decay, Rep. Prog. Phys. 75 (2012) 106301.

[16] LSND collaboration, A. Aguilar, L. B. Auerbach, R. L. Burman, D. O. Caldwell, E. D. Church, A. K. Cochran et al., Evidence for neutrino oscillations from the observation of $\bar{v}_{e}$ appearance in a $\bar{v}_{\mu}$ beam, Phys. Rev. D 64 (Nov, 2001) 112007.

[17] MiniBooNE collaboration, A. A. Aguilar-Arevalo et al., Improved search for $\bar{v}_{\mu} \rightarrow \bar{v}_{e}$ oscillations in the MiniBooNE experiment, Phys. Rev. Lett. 110 (2013) 161801, [1303.2588].

[18] G. Mention, M. Fechner, T. Lasserre, T. A. Mueller, D. Lhuillier, M. Cribier et al., Reactor antineutrino anomaly, Phys. Rev. D 83 (Apr, 2011) 073006.

[19] M. Bass, The short baseline neutrino oscillation program at Fermilab, PoS ICHEP2016 (2016) 481, [1702.00990].

[20] G. F. Knoll, Radiation Detection and Measurement. John Wiley \& Sons, 2010.

[21] J. D. Jackson, Classical electrodynamics. Wiley, New York, NY, 3rd ed., 1999.

[22] C. Giunti and C. W. Kim, Fundamentals of Neutrino Physics and Astrophysics. Oxford University Press, Oxford, UK, 2007.

[23] D. R. Nygren, A time projection chamber: 1975, in Proceedings, 1975 PEP Summer Study, Berkeley, July 28-August 20, 1975, pp. 126-133, 1975.

[24] A. R. Clark et al., Proposal for a PEP facility based on the time projection chamber, Tech. Rep. PEP-PROPOSAL-004, SLAC, 1976.

[25] G. Charpak, R. Bouclier, T. Bressani, J. Favier and C. Zupancic, The use of multiwire proportional counters to select and localize charged particles, Nucl. Instrum. Meth. 62 (1968) 262-268.

[26] T. Bressani, G. Charpak, D. Rahm and C. Zupancic, Track localization by means of a drift chamber, in Proc. of the Int. Seminar on Filmless Spark and Streamer Chambers, Dubna, Russian Federation, p. 275, 1969.

[27] A. H. Walenta, J. Heintze and B. Schuerlein, The multiwire drift chamber, a new type of proportional wire chamber, Nucl. Instrum. Meth. 92 (1971) 373-380.

[28] L. W. Alvarez, The use of liquid noble gases in particle detectors with 1. high spatial resolution over a large area, and 2. high-energy resolution as total absorption counters, Tech. Rep. UCRL-AGM-672, Lawrence Radiation Laboratory, 1968.

[29] N. Davidson and A. E. Larsh, Conductivity pulses in liquid argon, Phys. Rev. 74 (Jul, 1948) 220-220. 
[30] G. W. Hutchinson, Ionization in liquid and solid argon, Nature 162 (1948) 610-611.

[31] C. Rubbia, The liquid argon time projection chamber: A new concept for neutrino detectors, Tech. Rep. CERN-EP-INT-77-08, CERN, 1977.

[32] R. Acciarri, C. Adams, R. An, A. Aparicio, S. Aponte, J. Asaadi et al., Design and construction of the MicroBooNE detector, JINST 12 (2017) P02017.

[33] H. J. Hilke, Time projection chambers, Rep. Prog. Phys. 73 (2010) 116201.

[34] WA105 collaboration, A. Tonazzo, WA105: a large-scale demonstrator of the liquid argon double phase TPC, J. Phys. Conf. Ser. 718 (2016) 062061.

[35] E. Aprile, A. E. Bolotnikov, A. I. Bolozdynya and T. Doke, Noble Gas Detectors. Wiley-VCH Verlag GmbH \& Co. KGaA, 2006, 10.1002/9783527610020.

[36] DUNE collaboration, R. Acciarri et al., Long-Baseline Neutrino Facility (LBNF) and Deep Underground Neutrino Experiment (DUNE), 1601.02984.

[37] O. Bunemann, T. E. Cranshaw and J. A. Harvey, Design of grid ionization chambers, Can. J. Res. 27A (1949) 191.

[38] P. K. Lightfoot, G. J. Barker, K. Mavrokoridis, Y. A. Ramachers and N. J. C. Spooner, Optical readout tracking detector concept using secondary scintillation from liquid argon generated by a thick gas electron multiplier, JINST 4 (2009) P04002.

[39] A. Bressan, A. Buzulutskov, L. Ropelewski, F. Sauli and L. Shekhtman, High gain operation of GEM in pure argon, Nucl. Instrum. Meth. A423 (1999) 119 - 124.

[40] A. Rubbia, Experiments for CP violation: A Giant liquid argon scintillation, Cerenkov and charge imaging experiment?, in Proceedings of the 2nd NO-VE International Workshop on Neutrino Oscillations: Venice, December 3-5, 2003, pp. 321-350, 2004. hep-ph/0402110.

[41] A. Rubbia, ArDM: a ton-scale liquid argon experiment for direct detection of dark matter in the universe, J. Phys. Conf. Ser. 39 (2006) 129.

[42] C. E. Aalseth et al., The DarkSide multiton detector for the direct dark matter search, Adv. High Energy Phys. 2015 (2015) 541362.

[43] A. Badertscher, A. Curioni, U. Degunda, L. Epprecht, A. Gendotti, S. Horikawa et al., First operation and performance of a 200 lt double phase LAr LEM-TPC with a $40 \times$ $76 \mathrm{~cm} 2$ readout, JINST 8 (2013) P04012.

[44] ArDM collaboration, C. Amsler, A. Badertscher, V. Boccone, A. Bueno, M. C. Carmona-Benitez, W. Creus et al., First results on light readout from the 1-ton ArDM liquid argon detector for dark matter searches, JINST 5 (2010) P11003.

[45] S. Ramo, Currents induced by electron motion, Proc. of the IRE 27 (Sept, 1939) 584-585.

[46] W. Shockley, Currents to conductors induced by a moving point charge, J. App. Phys. 9 (1938) 635-636, [http://dx . doi .org/10.1063/1.1710367]. 
[47] MicroBooNE collaboration, A method to extract the charge distribution arriving at the TPC wire planes in MicroBooNE, Tech. Rep. MICROBOONE-NOTE-1017-PUB v1.0, FNAL, 2016.

[48] S. S.-S. Huang and G. R. Freeman, Electron transport in gaseous and liquid argon: Effects of density and temperature, Phys. Rev. A 24 (1981) .

[49] S. Amerio, S. Amoruso, M. Antonello, P. Aprili, M. Armenante, F. Arneodo et al., Design, construction and tests of the ICARUS T600 detector, Nucl. Instrum. Meth. A527 (2004) 329 - 410.

[50] S. Amoruso, M. Antonello, P. Aprili, F. Arneodo, A. Badertscher, B. Baiboussinov et al., Analysis of the liquid argon purity in the ICARUS T600 TPC, Nucl. Instrum. Meth. A516 (2004) 68 - 79.

[51] ARDM collaboration, J. Calvo et al., Measurement of the attenuation length of argon scintillation light in the ArDM LAr TPC, 1611.02481.

[52] Particle Data Group collaboration, K. A. Olive et al., Review of Particle Physics, Chin. Phys. C38 (2014) 090001.

[53] M. Miyajima, T. Takahashi, S. Konno, T. Hamada, S. Kubota, H. Shibamura et al., Average energy expended per ion pair in liquid argon, Phys. Rev. A9 (1974) $1438-1443$.

[54] T. Doke, K. Masuda and E. Shibamura, Estimation of absolute photon yields in liquid argon and xenon for relativistic (1 MeV) electrons, Nucl. Instrum. Meth. A291 (1990) 617-620.

[55] J. Jortner, L. Meyer, S. A. Rice and E. G. Wilson, Localized excitations in condensed Ne, Ar, Kr, and Xe, J. Chem. Phys. 42 (1965) 4250-4253, [http://dx.doi.org/10.1063/1.1695927].

[56] T. Heindl, T. Dandl, M. Hofmann, R. Krücken, L. Oberauer, W. Potzel et al., The scintillation of liquid argon, EPL (Europhysics Letters) 91 (2010) 62002.

[57] A. Hitachi, T. Takahashi, N. Funayama, K. Masuda, J. Kikuchi and T. Doke, Effect of ionization density on the time dependence of luminescence from liquid argon and xenon, Phys. Rev. B 27 (May, 1983) 5279-5285.

[58] M. A. Zucker, A. R. Kishore, R. Sukumar and R. A. Dragoset, Elemental data index, NIST Standard Reference Database 105 (2015).

[59] I. Badhrees, A. Ereditato, I. Kreslo, M. Messina, U. Moser, B. Rossi et al., Measurement of the two-photon absorption cross-section of liquid argon with a time projection chamber, New J. Phys. 12 (2010) 113024.

[60] W. F. Schmidt, Electronic conduction processes in dielectric liquids, IEEE Trans. Elec. Insul. EI-19 (Oct, 1984) 389-418.

[61] A. C. Sinnock and B. L. Smith, Refractive indices of the condensed inert gases, Phys. Rev. 181 (May, 1969) 1297-1307. 
[62] G. M. Seidel, R. E. Lanou and W. Yao, Rayleigh scattering in rare gas liquids, Nucl. Instrum. Meth. A489 (2002) 189-194, [hep-ex/0111054].

[63] P. J. Linstrom and W. G. Mallard, NIST Chemistry WebBook, NIST Standard Reference Database 69 (2017) .

[64] C. Tegeler, R. Span and W. Wagner, A new equation of state for argon covering the fluid region for temperatures from the melting line to $700 \mathrm{~K}$ at pressures up to 1000 MPa, Journal of Physical and Chemical Reference Data 28 (1999) 779-850, [http://dx.doi.org/10.1063/1.556037].

[65] Y. Li et al., Measurement of longitudinal electron diffusion in liquid argon, Nucl. Instrum. Meth. A816 (2016) 160-170, [1508.07059].

[66] H. T. Davis, S. A. Rice and L. Meyer, On the kinetic theory of simple dense fluids. XI. experimental and theoretical studies of positive ion mobility in liquid $\mathrm{Ar}, \mathrm{Kr}$, and $\mathrm{Xe}, \mathrm{J}$. Chem. Phys. 37 (1962) 947-956, [http://dx.doi .org/10.1063/1.1733251].

[67] R. S. Mulliken, Potential curves of diatomic rare-gas molecules and their ions, with particular reference to Xe 2, J. Chem. Phys. 52 (1970) 5170-5180, [http://dx.doi.org/10.1063/1.1672756].

[68] T. Takahashi, S. Konno, T. Hamada, M. Miyajima, S. Kubota, A. Nakamoto et al., Average energy expended per ion pair in liquid xenon, Phys. Rev. A 12 (Nov, 1975) 1771-1775.

[69] J. Lekner, Motion of electrons in liquid argon, Phys. Rev. 158 (Jun, 1967) 130-137.

[70] M. H. Cohen and J. Lekner, Theory of hot electrons in gases, liquids, and solids, Phys. Rev. 158 (Jun, 1967) 305-309.

[71] J. P. Hernandez, Electron self-trapping in liquids and dense gases, Rev. Mod. Phys. 63 (Jul, 1991) 675-697.

[72] D. W. Swan, Drift velocity of electrons in liquid argon, and the influence of molecular impurities, Proc. Phys. Soc. 83 (1964) 659.

[73] H. Schnyders, S. A. Rice and L. Meyer, Electron mobilities in liquid argon, Phys. Rev. Lett. 15 (Aug, 1965) 187-190.

[74] H. D. Pruett and H. P. Broida, Free-carrier drift-velocity studies in rare-gas liquids and solids, Phys. Rev. 164 (Dec, 1967) 1138-1144.

[75] L. Miller and W. Spear, Charge transport in solid and liquid argon, Phys. Lett. A 24 (1967) $47-48$.

[76] J. A. Jahnke, L. Meyer and S. A. Rice, Zero-field mobility of an excess electron in fluid argon, Phys. Rev. A 3 (Feb, 1971) 734-752.

[77] K. Yoshino, U. Sowada and W. F. Schmidt, Effect of molecular solutes on the electron drift velocity in liquid Ar, Kr, and Xe, Phys. Rev. A 14 (Jul, 1976) 438-444. 
[78] E. Gushchin, A. Kruglov, A. Lebedev and I. Obodovskii, Drift of electrons in condensed argon, J. Exp. and Th. Phys. 51 (1980) 775.

[79] E. Aprile, Long-distance drifting of ionization electrons in liquid and solid argon, Il Nuovo Cimento C 9 (Mar, 1986) 621-635.

[80] K. Shinsaka, M. Codama, T. Srithanratana, M. Yamamoto and Y. Hatano, Electron-ion recombination rate constants in gaseous, liquid, and solid argon, J. Chem. Phys. 88 (1988) 7529-7536, [http: //dx. doi .org/10.1063/1.454317].

[81] E. Buckley et al., A study of ionization electrons drifting over large distances in liquid argon, Nucl. Instrum. Meth. A275 (1989) 364-372.

[82] A. M. Kalinin, Y. K. Potrebennikov, A. Gonidec and D. Schinzel, Temperature and electric field strength dependence of electron drift velocity in liquid argon, Tech. Rep. ATLAS Internal Note LARG-NO-058, CERN, 1996.

[83] W. Walkowiak, Drift velocity of free electrons in liquid argon, Nucl. Instrum. Meth. A449 (2000) 288 - 294.

[84] F. Bloch and N. E. Bradbury, On the mechanism of unimolecular electron capture, Phys. Rev. 48 (Oct, 1935) 689-695.

[85] G. Bakale, U. Sowada and W. F. Schmidt, Effect of an electric field on electron attachment to sulfur hexafluoride, nitrous oxide, and molecular oxygen in liquid argon and xenon, J. Phys. Chem. 80 (1976) 2556-2559, [http://dx.doi.org/10.1021/j100564a006].

[86] D. W. Swan, Electron attachment processes in liquid argon containing oxygen or nitrogen impurity, Proc. Phys. Soc. 82 (1963) 74.

[87] A. Bettini et al., A study of the factors affecting the electron lifetime in ultrapure liquid argon, Nucl. Instrum. Meth. A305 (1991) 177-186.

[88] W. Hofmann, U. Klein, M. Schulz, J. Spengler and D. Wegener, Production and transport of conduction electrons in a liquid argon ionization chamber, Nucl. Instrum. Meth. 135 (1976) 151.

[89] S. D. Biller, D. Kabat, R. C. Allen, G. Bühler and P. J. Doe, Effects of oxygen and nitrogen on drifting electrons in a liquid argon TPC, Nucl. Instrum. Meth. A276 (1989) $144-150$.

[90] A. S. Barabash and A. I. Bolozdynya, Liquid Ionization Detectors (in Russian). EnergoAtomizdat, Moscow, 1993.

[91] S. Palestini, G. Barr, C. Biino, P. Calafiura, A. Ceccucci, C. Cerri et al., Space charge in ionization detectors and the NA48 electromagnetic calorimeter, Nucl. Instrum. Meth. A421 (1999) 75 - 89.

[92] A. Einstein, Über die von der molekularkinetischen theorie der wärme geforderte bewegung von in ruhenden flüssigkeiten suspendierten teilchen, Ann. der Phys. 322 (1905) 549-560. 
[93] M. von Smoluchowski, Zur kinetischen theorie der brownschen molekularbewegung und der suspensionen, Ann. der Phys. 326 (1906) 756-780.

[94] P. Cennini, S. Cittolin, J.-P. Revol, C. Rubbia, W.-H. Tian, P. Picchi et al., Performance of a three-ton liquid argon time projection chamber, Nucl. Instrum. Meth. A345 (1994) 230 - 243.

[95] E. Shibamura, T. Takahashi, S. Kubota and T. Doke, Ratio of diffusion coefficient to mobility for electrons in liquid argon, Phys. Rev. A 20 (Dec, 1979) 2547-2554.

[96] S. Derenzo, A. Kirschbaum, P. Eberhard, R. Ross and F. Solmitz, Test of a liquid argon chamber with 20 um RMS resolution, Nucl. Instrum. and Meth. 122 (1974) 319 - 327.

[97] L. Onsager, Initial recombination of ions, Phys. Rev. 54 (Oct, 1938) 554-557.

[98] G. Jaffé, Zur theorie der ionisation in kolonnen, Ann. der Phys. 347 (1913) 303-344.

[99] J. Thomas and D. A. Imel, Recombination of electron-ion pairs in liquid argon and liquid xenon, Phys. Rev. A 36 (Jul, 1987) 614-616.

[100] S. Amoruso, M. Antonello, P. Aprili, F. Arneodo, A. Badertscher, B. Baiboussinov et al., Study of electron recombination in liquid argon with the ICARUS TPC, Nucl. Instrum. Meth. A523 (2004) 275 - 286.

[101] R. Acciarri, C. Adams, J. Asaadi, B. Baller, T. Bolton, C. Bromberg et al., A study of electron recombination using highly ionizing particles in the ArgoNeuT liquid argon TPC, JINST 8 (2013) P08005.

[102] U. Fano, Ionization yield of radiations. II. The fluctuations of the number of ions, Phys. Rev. 72 (Jul, 1947) 26-29.

[103] T. Doke, Fundamental properties of liquid argon, krypton and xenon as radiation detector media, Portugal. Phys. 12 (1981) 9-48.

[104] T. Doke, A. Hitachi, S. Kubota, A. Nakamoto and T. Takahashi, Estimation of Fano factors in liquid argon, krypton, xenon and xenon-doped liquid argon, Nucl. Instrum. Meth. 134 (1976) 353-357.

[105] E. Aprile, J. Park, H. Schwartz and W. H.-M. Ku, Energy resolution studies of liquid argon ionization detectors, Nucl. Instrum. Meth. A261 (1987) 519-526.

[106] J. Thomas, D. A. Imel and S. Biller, Statistics of charge collection in liquid argon and liquid xenon, Phys. Rev. A 38 (Dec, 1988) 5793-5800.

[107] WARP collaboration, P. Benetti et al., Measurement of the specific activity of Ar-39 in natural argon, Nucl. Instrum. Meth. A574 (2007) 83-88, [astro-ph/0603131].

[108] B. Singh and J. A. Cameron, Nuclear data sheets for A =39, Nuclear Data Sheets 107 (Feb., 2006) 225-354.

[109] E. Aprile, W. H. Ku and J. Park, Delta electron production and the ultimate energy resolution of liquid argon ionization detectors, IEEE Trans. Nucl. Sci. 35 (Feb, 1988) $37-41$. 
[110] F. Arneodo et al., Observation of long ionizing tracks with the ICARUS T600 first half-module, Nucl. Instrum. Meth. A508 (2003) 287-294.

[111] S. Bonetti et al., A study of the electron image due to ionizing events in a two-dimensional liquid argon TPC with a $24 \mathrm{~cm}$ drift gap, Nucl. Instrum. Meth. A286 (1990) 135.

[112] O. Palamara, Event Reconstruction in LAr-TPC, AIP Conf. Proc. 1222 (2010) $122-126$.

[113] ICARUS collaboration, M. Antonello et al., Search for anomalies in the $v_{e}$ appearance from a $v_{\mu}$ beam, Eur. Phys. J. C73 (2013) 2599, [1307 . 4699].

[114] M. Antonello et al., Experimental observation of an extremely high electron lifetime with the ICARUS-T600 LAr-TPC, JINST 9 (2014) P12006, [1409. 5592].

[115] M. Antonello et al., Precise 3D track reconstruction algorithm for the ICARUS T600 liquid argon time projection chamber detector, Adv. High Energy Phys. 2013 (2013) 260820, [1210.5089].

[116] M. Antonello, B. Baibussinov, P. Benetti, F. Boffelli, A. Bubak, E. Calligarich et al., The trigger system of the ICARUS experiment for the CNGS beam, JINST 9 (2014) P08003.

[117] M. Antonello, B. Baibussinov, P. Benetti, F. Boffelli, E. Calligarich, N. Canci et al., Precision measurement of the neutrino velocity with the ICARUS detector in the CNGS beam, J. High Energy Phys. 2012 (Nov, 2012) 49.

[118] C. Anderson et al., The ArgoNeuT detector in the NuMI low-energy beam line at Fermilab, JINST 7 (2012) P10019, [1205 . 6747].

[119] ArgoNeuT collaboration, C. Anderson, M. Antonello, B. Baller, T. Bolton, C. Bromberg, F. Cavanna et al., First measurements of inclusive muon neutrino charged current differential cross sections on argon, Phys. Rev. Lett. 108 (Apr, 2012) 161802.

[120] ArgoNeuT collaboration, R. Acciarri, C. Adams, J. Asaadi, B. Baller, T. Bolton, C. Bromberg et al., Measurements of inclusive muon neutrino and antineutrino charged current differential cross sections on argon in the NuMI antineutrino beam, Phys. Rev. D 89 (Jun, 2014) 112003.

[121] R. Acciarri, C. Adams, J. Asaadi, B. Baller, T. Bolton, C. Bromberg et al., Detection of back-to-back proton pairs in charged-current neutrino interactions with the ArgoNeuT detector in the NuMI low energy beam line, Phys. Rev. D 90 (Jul, 2014) 012008.

[122] C. Anderson, M. Antonello, B. Baller, T. Bolton, C. Bromberg, F. Cavanna et al., Analysis of a large sample of neutrino-induced muons with the ArgoNeuT detector, JINST 7 (2012) P10020.

[123] ArgoNeuT collaboration, R. Acciarri et al., First measurement of neutrino and antineutrino coherent charged pion production on argon, Phys. Rev. Lett. 113 (2014) 261801, [1408.0598]. 
[124] E. D. Church, LArSoft: A software package for liquid argon time projection drift chambers, 1311.6774.

[125] LARIAT collaboration, F. Cavanna, M. Kordosky, J. Raaf and B. Rebel, LArIAT: Liquid argon in a testbeam, 1406.5560.

[126] R. Acciarri, C. Adams, R. An, J. Asaadi, M. Auger, L. Bagby et al., Convolutional neural networks applied to neutrino events in a liquid argon time projection chamber, JINST 12 (2017) P03011.

[127] MicroBooNE collaboration, R. Acciarri et al., Michel electron reconstruction using cosmic-ray data from the MicroBooNE LArTPC, Submitted to: JINST (2017), [1704.02927].

[128] A. Ereditato, C. C. Hsu, S. Janos, I. Kreslo, M. Messina, C. Rudolf von Rohr et al., Design and operation of ARGONTUBE: a 5 m long drift liquid argon TPC, JINST 8 (2013) P07002, [1304.6961].

[129] DUNE collaboration, B. Abi et al., The single-phase ProtoDUNE technical design report, In Preparation (2017).

[130] J. Lewin and P. Smith, Review of mathematics, numerical factors, and corrections for dark matter experiments based on elastic nuclear recoil, Astroparticle Phys. 6 (1996) $87-112$.

[131] A. Badertscher, A. Curioni, L. Knecht, D. Lussi, A. Marchionni, G. Natterer et al., First operation of a double phase LAr large electron multiplier time projection chamber with a 2D projective readout anode, Nucl. Instrum. Meth. A641 (2011) 48 57.

[132] C. Cantini et al., Long-term operation of a double phase LAr LEM Time Projection Chamber with a simplified anode and extraction-grid design, JINST 9 (2014) P03017, [1312.6487].

[133] D. Montanari, M. Adamowski, A. Hahn, B. Norris, J. Reichenbacher, R. Rucinski et al., Performance and results of the LBNE 35 ton membrane cryostat prototype, Phys. Procedia 67 (2015) 308-313.

[134] LBNE collaboration, D. Montanari et al., First scientific application of the membrane cryostat technology., AIP Conf. Proc. 1573 (2014) 1664-1671.

[135] DUNE 35-ton РRототуре collaboration, Design and performance of the DUNE 35-ton prototype time projection chamber (in preparation), Nucl. Instrum. Meth. A (2018) .

[136] M. Adamowski et al., The liquid argon purity demonstrator, JINST 9 (2014) P07005, [1403. 7236].

[137] P. Benetti et al., Argon purification in the liquid phase, Nucl. Instrum. Meth. A333 (1993) 567-570. 
[138] K. Mavrokoridis, R. G. Calland, J. Coleman, P. K. Lightfoot, N. McCauley, K. J. McCormick et al., Argon purification studies and a novel liquid argon re-circulation system, JINST 6 (2011) P08003, [1106.5226].

[139] G. Bressi, M. Cambiaghi, G. Carugno, N. Toniolo, E. Conti, B. Dainese et al., Argon purification in liquid phase, Nucl. Instrum. Meth. A292 (1990) 585-592.

[140] P. J. Doe, R. C. Allen, S. D. Biller, G. Buhler, W. A. Johnson and H. H. Chen, A large scale purification system for a liquid argon time projection chamber, Nucl. Instrum. Meth. A258 (1987) 170-176.

[141] A. Curioni, B. T. Fleming, W. Jaskierny, C. Kendziora, J. Krider, S. Pordes et al., A regenerable filter for liquid argon purification, Nucl. Instrum. Meth. A605 (2009) 306-311, [0903.2066].

[142] B. Baibussinov et al., Free electron lifetime achievements in liquid argon imaging TPC, JINST 5 (2010) P03005, [0910 . 5087].

[143] LBNE collaboration, A. Hahn, M. Adamowski, D. Montanari, B. Norris, J. Reichenbacher, R. Rucinski et al., The LBNE 35 ton prototype cryostat, in Proceedings, 21st Symposium on Room-Temperature Semiconductor X-ray and Gamma-ray Detectors (RTSD 2014): Seattle, WA, USA, November 8-15, 2014, p. 7431158, 2016.

[144] K. Biery, C. Green, J. Kowalkowski, M. Paterno and R. Rechenmacher, artdaq: An event-building, filtering, and processing framework, IEEE Trans. Nucl. Sci. 60 (2013) $3764-3771$.

[145] R. Brun and F. Rademakers, ROOT: An object oriented data analysis framework, in New computing techniques in physics research V. Proceedings, 5th International Workshop, AIHENP '96, Lausanne, Switzerland, September 2-6, 1996, vol. A389, pp. 81-86, 1997.

[146] J. Insler, Photon detection system performance in the DUNE 35 ton prototype LAr-TPC detector, In Preparation (2017) .

[147] N. McConkey, N. Spooner, M. Thiesse, M. Wallbank and T. K. Warburton, Cryogenic CMOS cameras for high voltage monitoring in liquid argon, JINST 12 (2017) P03014, [1612.06124].

[148] D. W. Swan and T. J. Lewis, The influence of cathode and anode surfaces on the electric strength of liquid argon, Proc. Phys. Soc. 78 (1961) 448.

[149] M. Auger, A. Blatter, A. Ereditato, D. Goeldi, S. Janos, I. Kreslo et al., On the electric breakdown in liquid argon at centimeter scale, JINST 11 (2016) P03017, [1512.05968].

[150] MicroBooNE collaboration, R. Acciarri et al., Liquid argon dielectric breakdown studies with the MicroBooNE purification system, JINST 9 (2014) P11001, [1408.0264]. 
[151] LBNE collaboration, D. Gong et al., The cryogenic performances of specific optical and electrical components for a liquid argon time projection chamber, Phys. Procedia 37 (2012) 1654.

[152] R. L. Patterson, A. Hammoud and M. Elbulu, Assessment of electronics for cryogenic space exploration missions, Cryogenics 46 (2006) 231.

[153] Y. Creten et al., An 8-bit flash analog-to-digital converter in standard CMOS technology functional from 4.2 K to 300 K, IEEE J. Solid-State Circuits 44 (2009) 2019.

[154] E. Takeda, N. Suzuki and T. Hagiwara, Device performance degradation to hot-carrier injection at energies below the $\mathrm{Si}_{-} \mathrm{SiO}_{2}$ energy barrier, Proc. Intl. Electron Devices Meeting (1983) 396.

[155] F. Bay, C. Cantini, S. Murphy, F. Resnati, A. Rubbia, F. Sergiampietri et al., Evidence of electric breakdown induced by bubbles in liquid argon, in High Voltage in Noble Liquids Workshop, 2014. 1401.2777.

[156] https://web.fnal.gov/collaboration/DUNE/Images\%20for\%20Web/ LAr-in-DUNE-prototype.gif.

[157] C. Green, J. Kowalkowski, M. Paterno, M. Fischler, L. Garren and Q. Lu, The art framework, J. Phys. Conf. Ser. 396 (2012) 022020.

[158] GEANT4 collaboration, S. Agostinelli et al., GEANT4: A simulation toolkit, Nucl. Instrum. Meth. A506 (2003) 250-303.

[159] https://cdcvs.fnal.gov/redmine/projects/dunetpc.

[160] R. Putz, Specification of the Fermilab hierarchical configuration language, Tech. Rep. Unpublished, FNAL, 2012.

[161] C. Hagmann, D. Lange and D. Wright, Cosmic-ray shower generator (CRY) for Monte Carlo transport codes, in 2007 IEEE Nuclear Science Symposium Conference Record, vol. 2, pp. 1143-1146, Oct, 2007.

[162] D. Adams, "Bad channels for 35t." 35-ton Simulation, Reconstruction, and Analysis Weekly Meeting, 11 Jan. 2017.

[163] J. Insler, dunetpc/dune/CalData/UnstickADCCodes_module.cc, 2015.

[164] B. Kirby, dunetpc/dune/daqinput35t/FilterWF_module.cc, 2016.

[165] B. Baller, Liquid argon TPC signal formation, signal processing and hit reconstruction, 1703.04024 .

[166] T. K. Warburton, dunetpc/dune/daqinput35t/Sigmoidfilter_module.cc, 2016.

[167] W. H. Press, S. A. Teukolsky, W. T. Vetterling and B. P. Flannery, Numerical Recipes 3rd Edition: The Art of Scientific Computing. Cambridge University Press, New York, NY, USA, 3 ed., 2007. 
[168] MicroBooNE collaboration, J. Asaadi, Gauss Hit Finder: Hit finding for the MicroBooNE TPC, Tech. Rep. Internal MicroBooNE DocDB 3843, November, 2014.

[169] P. H. S. Torr and A. Zisserman, MLESAC: A new robust estimator with application to estimating image geometry, Computer Vision and Image Understanding 78 (April, 2000) 138-156.

[170] M. A. Fischler and R. C. Bolles, Random Sample Consensus: A paradigm for model fitting with applications to image analysis and automated cartography, Communications of the ACM 24 (June, 1981) 381-395.

[171] A. P. Dempster, N. M. Laird and D. B. Rubin, Maximum likelihood from incomplete data via the EM algorithm, J. Royal Statistical Society. Series B (Methodological) 39 (1977) 1-38.

[172] L. Landau, On the energy loss of fast particles by ionization, J. Phys. USSR 8 (1944) 201.

[173] C. Bromberg et al., Design and operation of LongBo: a $2 \mathrm{~m}$ long drift liquid argon TPC, JINST 10 (2015) P07015, [1504.00398].

[174] W. Verkerke and D. P. Kirkby, The RooFit toolkit for data modeling, in Statistical Problems in Particle Physics, Astrophysics and Cosmology (PHYSTAT 05):

Proceedings, Oxford, UK, September 12-15, 2005, vol. C0303241, p. MOLT007, 2003. physics/0306116.

[175] F. James, Minuit: Function minimization and error analysis. reference manual, version 94.1, Tech. Rep. CERN Program Library Entry D506, CERN, 1994.

[176] K. Kölbig and B. Schorr, A program package for the landau distribution, Computer Physics Communications 31 (1984) 97 - 111.

[177] M. Paterno, Calculating efficiencies and their uncertainties, Tech. Rep. http://home.fnal.gov/ paterno/images/effic.pdf, FNAL, 2003.

[178] R. Carroll, D. Ruppert, L. Stefanski and C. Crainiceanu, Measurement Error in Nonlinear Models: A Modern Perspective, Second Edition. Chapman \& Hall/CRC Monographs on Statistics \& Applied Probability. CRC Press, 2006.

[179] M. H. Kutner, C. J. Nachtsheim and J. Neter, Applied Linear Regression Models. McGraw-Hill, New York, NY, 4th ed., 2003.

[180] MicroBooNE collaboration, A measurement of the attenuation of drifting electrons in the MicroBooNE LArTPC, Tech. Rep. MICROBOONE-NOTE-1026-PUB, FNAL, 2017.

[181] G. J. Michna, S. P. Gent and A. Propst, CFD analysis of fluid, head, and impurity flows in DUNE far detector to address additional design considerations, Tech. Rep. DUNE DocDB 3213, Mechanical Engineering Department, South Dakota State University, 2017. 
[182] MicroBooNE collaboration, Study of space charge effects in MicroBooNE, Tech. Rep. MICROBOONE-NOTE-1018-PUB, FNAL, 2016.

[183] M.-L. Gallin-Martel, L. Gallin-Martel, Y. Grondin, O. Rossetto, J. Collot, D. Grondin et al., A liquid xenon positron emission tomograph for small animal imaging: First experimental results of a prototype cell, Nucl. Instrum. Meth. A599 (2009) 275 - 283.

[184] A. Breskin, R. Alon, M. Cortesi, R. Chechik, J. Miyamoto, V. Dangendorf et al., A concise review on THGEM detectors, Nucl. Instrum. Meth. A598 (2009) 107-111, [0807.2026].

[185] B. L. Paredes, C. D. R. Azevedo, S. Paganis, A. L. M. Silva, N. J. C. Spooner and J. F. C. A. Veloso, Cryogenic gaseous photomultiplier for position reconstruction of liquid argon scintillation light, JINST 10 (2015) P07017, [1501 . 05674].

[186] Y. Li, C. Thorn, W. Tang, J. Joshi, X. Qian, M. Diwan et al., A 20-liter test stand with gas purification for liquid argon research, JINST 11 (2016) T06001.

[187] Y. Li, J. Sondericker and C. Thorn, Omega group noble liquid experiments: A discussion of LAr time projection chamber (TPC) technology and cryogenic operating procedures for our TPC experiments, Tech. Rep. v. 2.0, Brookhaven National Laboratory, 2013.

[188] DUNE collaboration, J. Strait et al., Long-Baseline Neutrino Facility (LBNF) and Deep Underground Neutrino Experiment (DUNE), 1601.05823.

[189] R. Andrews, W. Jaskierny, H. Jöstlein, C. Kendziora, S. Pordes and T. Tope, A system to test the effect of materials on electron drift lifetime in liquid argon and the effect of water, Nucl. Instrum. Meth. A608 (2009) 251 - 258.

[190] D. Gastler, E. Kearns, A. Hime, L. C. Stonehill, S. Seibert, J. Klein et al., Measurement of scintillation efficiency for nuclear recoils in liquid argon, Phys. Rev. C 85 (Jun, 2012) 065811.

[191] G. Plante, E. Aprile, R. Budnik, B. Choi, K.-L. Giboni, L. W. Goetzke et al., New measurement of the scintillation efficiency of low-energy nuclear recoils in liquid xenon, Phys. Rev. C 84 (Oct, 2011) 045805.

[192] J. T. Bushberg, J. A. Seibert, E. M. Leidholdt, J. M. Boone and E. J. Goldschmidt, The essential physics of medical imaging, Medical Physics 30 (2003) 1936-1936.

[193] L. Periale, V. Peskov, C. Iacobaeus, B. Lund-Jensen, P. Pavlopoulos, P. Picchi et al., A study of the operation of especially designed photosensitive gaseous detectors at cryogenic temperatures, Nucl. Instrum. Meth. A567 (2006) 381 - 385.

[194] L. Arazi, A. E. C. Coimbra, E. Erdal, I. Israelashvili, M. L. Rappaport, S. Shchemelinin et al., First results of a large-area cryogenic gaseous photomultiplier coupled to a dual-phase liquid xenon TPC, JINST 10 (2015) P10020, [1508 . 00410].

[195] Y. Xie, H. Liu, A. Zhang, Y. Liu, T. Hu, L. Zhou et al., Quantum efficiency measurement of CsI photocathodes using synchrotron radiation at BSRF, Nucl. Instrum. Meth. A664 (2012) 310 - 316. 
[196] J. Veloso, C. Santos, F. Pereira, C. Azevedo, F. Amaro, J. dos Santos et al., THCOBRA: Ion back flow reduction in patterned THGEM cascades, Nucl. Instrum. Meth. A639 (2011) 134 - 136.

[197] H. Raether, Electron avalanches and breakdown in gases. Butterworths, 1964.

[198] M. S. Naidu and V. Kamaraju, High Voltage Engineering. Tata McGraw-Hill Education, 2004.

[199] R. Bouclier, M. Capeans, W. Dominik, M. Hoch, J. C. Labbe, G. Million et al., The gas electron multiplier (GEM), IEEE Trans. Nucl. Sci. 44 (Jun, 1997) 646-650.

[200] A. L. M. Silva, C. D. R. Azevedo, L. F. N. D. Carramate, T. Lopes, I. F. Castro, $\mathrm{R}$. de Oliveira et al., X-ray imaging detector based on a position sensitive THCOBRA with resistive line, JINST 8 (2013) P05016.

[201] H. N. da Luz, J. Veloso, N. Mendes, J. dos Santos and J. Mir, MHSP with position detection capability, Nucl. Instrum. Meth. A573 (2007) 191 - 194.

[202] A. Breskin, CsI UV photocathodes: history and mystery, Nucl. Instrum. Meth. A371 (1996) $116-136$.

[203] V. Dangendorf, A. Breskin, R. Chechik and H. Schmidt-Böcking, A gas-filled UV-photon detector with CsI photocathode for the detection of Xe light, Nucl. Instrum. Meth. A289 (1990) 322 - 324.

[204] P. Krizan, M. Cindro, V. Cindro, S. Korpar, G. Omahen, A. Stanovnik et al., Measurements of the quantum efficiency of CsI photocathodes in a multiwire proportional chamber, Nucl. Instrum. Meth. A364 (1995) 243-252.

[205] M. Thiesse, “AgMD2_GPM.” https://github.com/tz-m/AgMD2_GPM.

[206] M. Thiesse, "ProcessGPMPMT.” https://github.com/tz-m/ProcessGPMPMT.

[207] M. Thiesse, “CAENHV.” https://github.com/tz-m/CAENHV.

[208] M. Thiesse, “Do_GPM.” https://github.com/tz-m/Do_GPM.

[209] G. Alkhazov, Statistics of electron avalanches and ultimate resolution of proportional counters, Nucl. Instrum. Meth. 89 (1970) 155 - 165.

[210] C. Richter, A. Breskin, R. Chechik, D. Mörmann, G. Garty and A. Sharma, On the efficient electron transfer through GEM, Nucl. Instrum. Meth. A478 (2002) 538 - 558.

[211] I. B. Keirim-Markus, A. K. Savinsky, V. G. Tchaikovsky and A. S. Yakovlev, Relative luminescence yield of gas proportional counters, Instr. Exp. Tech. 15 (1972) 46-48.

[212] J. Birks, The Theory and Practice of Scintillation Counting. Pergamon, 1964.

[213] M. Forte, Light pulses excited by $\alpha$ particles in argon: A gaseous scintillation detector, Il Nuovo Cimento (1955-1965) 3 (Jun, 1956) 1443-1455. 
[214] NIST Cryogenics Technologies Group, "Material properties." http://cryogenics.nist.gov/MPropsMAY/materialproperties.htm.

[215] A. Buzulutskov, Advances in cryogenic avalanche detectors, JINST 7 (2012) C02025.

[216] R. Galea, J. Dodd, Y. Ju, M. Leltchouk, W. Willis, P. Rehak et al., Gas purity effect on GEM performance in He and Ne at low temperatures, IEEE Trans. Nucl. Sci. 53 (Aug, 2006) 2260-2263. 